SLAC-PUB-15972

\title{
Light-Front Holographic QCD and Emerging Confinement
}

\author{
Stanley J. Brodsky \\ SLAC National Accelerator Laboratory, Stanford University, Stanford, CA 94309, USA
}

Guy F. de Téramond

Universidad de Costa Rica, San José, Costa Rica

Hans Günter Dosch

Institut für Theoretische Physik, Philosophenweg 16, D-6900 Heidelberg, Germany

Joshua Erlich

College of William and Mary, Williamsburg, VA 23187, USA

sjbth@slac.stanford.edu, gdt@asterix.crnet.cr,

h.g.dosch@thphys.uni-heidelberg.de, jxerli@wm.edu

(Invited review article to appear in Physics Reports) 


\begin{abstract}
In this report we explore the remarkable connections between light-front dynamics, its holographic mapping to gravity in a higher-dimensional anti-de Sitter (AdS) space, and conformal quantum mechanics. This approach provides new insights into the origin of a fundamental mass scale and the physics underlying confinement dynamics in QCD in the limit of massless quarks. The result is a relativistic light-front wave equation for arbitrary spin with an effective confinement potential derived from a conformal action and its embedding in AdS space. This equation allows for the computation of essential features of hadron spectra in terms of a single scale. The light-front holographic methods described here gives a precise interpretation of holographic variables and quantities in AdS space in terms of light-front variables and quantum numbers. This leads to a relation between the AdS wave functions and the boost-invariant light-front wave functions describing the internal structure of hadronic bound-states in physical spacetime. The pion is massless in the chiral limit and the excitation spectra of relativistic light-quark meson and baryon bound states lie on linear Regge trajectories with identical slopes in the radial and orbital quantum numbers. In the light-front holographic approach described here currents are expressed as an infinite sum of poles, and form factors as a product of poles. At large $q^{2}$ the form factor incorporates the correct powerlaw fall-off for hard scattering independent of the specific dynamics and is dictated by the twist. At low $q^{2}$ the form factor leads to vector dominance. The approach is also extended to include small quark masses. We briefly review in this report other holographic approaches to QCD, in particular top-down and bottom-up models based on chiral symmetry breaking. We also include a discussion of open problems and future applications.
\end{abstract}




\section{Contents}

1 Introduction $\quad 2$

1.1 Motivation . . . . . . . . . . . . . . . . . . 2

1.2 The AdS/CFT correspondence and holographic QCD . . . . . . . . . 4

1.3 Light-front holographic QCD . . . . . . . . . . . . . . . 10

1.4 Confinement and conformal algebraic structures . . . . . . . . . . . . . 12

1.5 Other approaches and applications . . . . . . . . . . . . . . . . 14

1.6 Contents of this review . . . . . . . . . . . . . . . . . 15

2 A Semiclassical Approximation to Light-Front Quantized QCD 17

2.1 The Dirac forms of relativistic dynamics . . . . . . . . . . . . . 20

2.2 Light-front dynamics . . . . . . . . . . . . . . . . . . . 21

2.3 Light-front quantization of QCD . . . . . . . . . . . . . . . . . . . . . . . . . . . . .

2.3.1 Representation of hadrons in the light-front Fock basis . . . . . . 26

2.4 Semiclassical approximation to QCD in the light front . . . . . . . . . 29

2.4.1 Inclusion of light quark masses . . . . . . . . . . . . . . . 34

3 Conformal Quantum Mechanics and Light-Front Dynamics 37

3.1 One-dimensional conformal field theory . . . . . . . . . . . . . 38

3.2 Connection to light-front dynamics . . . . . . . . . . . . . . . 43

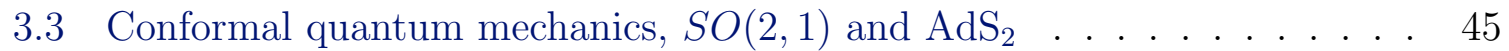

4 Higher-Spin Wave Equations and AdS Kinematics and Dynamics 47

4.1 Scalar and vector fields . . . . . . . . . . . . . . . . . 48

4.2 Arbitrary integer spin . . . . . . . . . . . . . . . 50

4.2.1 Confining interaction and warped metrics . . . . . . . . 55

4.2.2 Higher spin in a gauge invariant AdS model . . . . . . . . . . . 56

4.3 Arbitrary half-integer spin . . . . . . . . . . . . . . . . . . . 57 
5 Light-Front Holographic Mapping and Hadronic Spectrum $\quad 61$

5.1 Integer $\operatorname{spin} \ldots \ldots \ldots$. . . . . . . . . . . . . . . . . . 63

5.1.1 A light-front holographic model for mesons . . . . . . . . . . . 64

5.1.2 Meson spectroscopy in a gauge invariant AdS model . . . . . . . . 70

5.1.3 Light quark masses and meson spectrum . . . . . . . . . . . . 71

5.2 Half-integer spin . . . . . . . . . . . . . . . 76

5.2.1 A light-front holographic model for baryons . . . . . . . . . . . 78

6 Light-Front Holographic Mapping and Transition Amplitudes 85

6.1 Meson electromagnetic form factor . . . . . . . . . . . . 86

6.1.1 Meson form factor in AdS space . . . . . . . . . . . . . 86

6.1.2 Meson form factor in light-front QCD . . . . . . . . . . . 88

6.1.3 Light-front holographic mapping . . . . . . . . . . . . . . 89

6.1.4 Soft-wall form factor model . . . . . . . . . . . . . . . . . . 94

6.1.5 Time-like form factors in holographic QCD . . . . . . . . . . . 99

6.2 Nucleon electromagnetic form factors . . . . . . . . . . . . . . . . 103

6.2.1 Computing nucleon form factors in light-front holographic QCD . 105

7 Other Bottom-Up and Top-Down Holographic Models 110

7.1 Bottom-up models . . . . . . . . . . . . . . 111

7.2 A bottom-up model with chiral symmetry breaking . . . . . . . . . . . 119

7.3 Top-down models . . . . . . . . . . . . . . . . . . 122

7.4 The Sakai-Sugimoto model . . . . . . . . . . . . . . . . . . . 125

8 Summary and Conclusion $\quad 130$

8.1 Open problems and future applications . . . . . . . . . . . . 134

A Riemannian Geometry and Anti-de Sitter Space 142

A.1 Basics of non-Euclidean geometry . . . . . . . . . . . . . . . . . 142

A.1.1 Covariant derivative and parallel transport . . . . . . . . . . . . 144

A.1.2 Space-time curvature . . . . . . . . . . . . . 145

A.1.3 Spinors in non-Euclidean geometry . . . . . . . . . . . . . 147

A.2 Maximally symmetric spaces . . . . . . . . . . . . . . . . . 149

A.2.1 Definition . . . . . . . . . . . . . . . . 149

A.2.2 Anti-de Sitter space-time $\mathrm{AdS}_{d+1}$. . . . . . . . . . . . . 150

A.2.3 Relation between $\operatorname{Conf}\left(R^{1}\right), S O(2,1)$ and the isometries of $\mathrm{AdS}_{2} 153$ 
B Light-Front Metric Conventions and Spinors 156

$\begin{array}{lr}\text { C Notes on Conformal Quantum Mechanics } & 158\end{array}$

D Useful Formulas for Higher Spin Equations in Anti-de Sitter Space 162

D.1 Arbitrary integer spin . . . . . . . . . . . . . . . . . . 162

D.1.1 The action in the local Lorentz frame . . . . . . . . . . 162

D.1.2 Warped metric . . . . . . . . . . . . . . . . 164

D.2 Arbitrary half integer spin . . . . . . . . . . . . . . 165

D.2.1 General treatment . . . . . . . . . . . . . . . . 165

D.2.2 Spin- $\frac{3}{2}$ Rarita-Schwinger field in AdS space $\ldots \ldots \ldots \ldots$

E Light-Front Holographic Mapping and the Energy-Momentum Tensor

E.1 Gravitational form factor of composite hadrons . . . . . . . . . . . . 169

E.1.1 Meson gravitational form factor in AdS space . . . . . . . . . 170

E.1.2 Meson gravitational form factor in light-front QCD . . . . . . 173

E.1.3 Light-front holographic mapping . . . . . . . . . . . . . . 175

$\begin{array}{lll}\text { F } & \text { Propagators in the Limiting Theory of } \mathrm{AdS}_{5} & 177\end{array}$

F.1 AdS boundary conditions and gauge/gravity correspondence . . . . . . 177

F.2 Two-point functions for arbitrary spin and Migdal procedure . . . . . . 179

G Some Useful Formulæ 184

G.1 Solutions of the equations of motion in AdS space . . . . . . . . . 184

G.1.1 A useful integral . . . . . . . . . . . . . . . 187

H Integrability and Light-Front Effective Hamiltonians 189

H.1 Light-front effective bosonic Hamiltonians ～. . . . . . . . . . . . . 190

H.1.1 Light-front hard-wall model . . . . . . . . . . . . . . . . . . . 190

H.1.2 Light-front soft-wall model . . . . . . . . . . . . . . . . . . . 192

H.2 Light-front effective fermionic Hamiltonians . . . . . . . . . . . . 194

H.2.1 Light-front hard-wall model . . . . . . . . . . . . . . . . 195

H.2.2 Light-front soft-wall model . . . . . . . . . . . . . . . . . . 197

I Equations of Motion for p-Form Fields in AdS 200 


\section{Chapter 1}

\section{Introduction}

\subsection{Motivation}

Quantum Chromodynamics (QCD), the $S U(3)$ color gauge field theory of quarks and gluons, is the standard theory of strong interactions. High energy experiments, such as the deep inelastic electron-proton scattering pioneered at SLAC [1], which revealed the quark structure of the proton, and continued at DESY [2] to extremely short distances, have shown that the basic elementary interactions of quarks and gluons are remarkably well described by QCD [3]. Yet, because of its strong-coupling nature, it has been difficult to make precise predictions outside of its short-distance perturbative domain where it has been tested to high precision. Unlike Quantum Electrodynamics (QED), the fundamental theory of electrons and photons, the strong couplings of quarks and gluons at large-distances makes the calculation of hadronic properties, such as hadron masses, a very difficult problem to solve, notwithstanding that the fundamental QCD Lagrangian is well established. In particular, one has no analytical understanding of how quarks and gluons are permanently confined and how hadrons emerge as asymptotic states in this theory [4]. In fact, in the limit of massless quarks no scale appears in the QCD Lagrangian. The classical Lagrangian of QCD is thus invariant under conformal transformations $[5,6]$. Nonetheless, the quantum theory built upon this conformal theory displays color confinement, a mass gap, and asymptotic freedom. One then confronts a fundamental question: how does the mass scale which determines the masses of the lightquark hadrons, the range of color confinement, as well as the running of the coupling appear in QCD?

Euclidean lattice methods [7] provide an important first-principle numerical simula- 
tion of nonperturbative QCD. However, the excitation spectrum of hadrons represents a difficult challenge to lattice QCD due to the enormous computational complexity beyond ground-state configurations and the unavoidable presence of multi-hadron thresholds [8]. Furthermore, dynamical observables in Minkowski space-time are not obtained directly from Euclidean space lattice computations. Other methods, as for example the DysonSchwinger equations, have also led to many important insights, such as the infrared fixed-point behavior of the strong coupling constant and the pattern of dynamical quark mass generation $[9,10,11,12]$. In practice, however, these analyses have been limited to ladder approximation in Landau gauge.

A problem, common to all realistic relativistic quantum field theories, is especially flagrant in QCD: the only known analytically tractable treatment is perturbation theory, which obviously is not the most appropriate tool for solving a strongly interacting theory with permanently confined constituents. In fact, according to the Kinoshita-LeeNauenberg theorem, which applies to any order of perturbation theory, a description of confinement using perturbative QCD is not possible in a simple way [13, 14]. Thus, an important theoretical goal is to find an initial approximation to QCD in its strongly coupled regime relevant at large distances, which is both analytically tractable and can be systematically improved. In fact, even in weakly interacting theories, like QED, there is a need for semiclassical equations in order to treat bound states. The Schrödinger and Dirac equations play a central role in atomic physics, providing simple, but effective, first approximations of the spectrum and wave functions of bound states which can be systematically improved using the Bethe-Salpeter formalism [15] and including corrections for quantum fluctuations, such as the Lamb shift and vacuum polarization. A long-sought goal in hadron physics is to find a simple analytic first approximation to QCD, analogous to the Schrödinger equation of atomic physics. This task is particularly challenging since the formalism must be fully relativistic, give a good description of the hadron spectrum, and should also explain essential dynamical properties of hadrons. There are several indications that such a goal might well be within reach:

i) The quark model, based mainly on the Schrödinger equation with relativistic corrections is qualitatively very successful (See e.g., [16], Sect. 14).

ii) There are striking regularities in the hadronic spectra, notably Regge trajectories $[17,18]$, which show a linear relation between the squared mass and the intrinsic angular momentum of hadrons (See e.g., [19, 20]).

iii) There exists a convenient frame-independent Hamiltonian framework for treating 
bound-states in relativistic theories using light-front quantization. It is based on the front-form or relativistic dynamics [21], where initial conditions are specified in the light-cone null-plane $x^{0}+x^{3}=0$, not on the usual initial conditions at equal time, $x^{0}=0$.

As an effective theory, we expect also that the resulting model incorporates underlying symmetries of the QCD Lagrangian.

\subsection{The AdS/CFT correspondence and holographic QCD}

The search for semiclassical equations in QCD obtained a strong advance some 15 years ago by the Maldacena Conjecture [22]. Roughly speaking, the conjecture states that a quantum gauge field theory in 4 dimensions corresponds to a classical gravitational theory in 5 dimensions. In this type of correspondence the higher-dimensional gravitational theory is referred to as the holographic dual, or gravity dual, of the lowerdimensional quantum field theory. Holographic ideas in physical theories have their origin in the seminal work of Bekenstein and Hawking in the 1970s [23, 24], which led to the surprising conclusion that black holes are thermodynamic systems which radiate at a temperature which depends on the size of the black hole. The most unusual aspect of black-hole thermodynamics is that the entropy of a black hole is proportional to the area of its horizon, contrary to the typical situation in non-gravitational systems, in which entropy is an extensive quantity proportional to the volume of the system. The maximal entropy of a system is a measure of the number of degrees of freedom in that system, so the distinction between gravitational and non-gravitational systems appears to limit the number of degrees of freedom of a gravitational system to that of a non-gravitational system in one fewer spatial dimension. This idea was formalized as the holographic principle, which postulates that a gravitational system may indeed be equivalent to a non-gravitational system in one fewer dimension [25, 26].

The AdS/CFT correspondence between gravity on a higher-dimensional anti-de Sitter (AdS) space and conformal field theories (CFT) in a lower-dimensional spacetime [22], is an explicit realization of the holographic principle, and it remains a major focus of string theory research. This correspondence has led to a semiclassical gravity approximation for strongly-coupled quantum field theories, providing physical insights into its nonperturbative dynamics. In practice, it provides an effective gravity description in 
a $(d+1)$-dimensional AdS, or other curved space-time, in terms of a flat $d$-dimensional conformally-invariant quantum field theory defined on the AdS asymptotic boundary, the boundary theory. In the semiclassical approximation, the generating functional of the quantum field theory is given by the minimum of the classical action of the gravitational theory at the 4-dimensional asymptotic border of the 5-dimensional space [27, 28]. Thus, in principle, one can compute physical observables in a strongly coupled gauge theory in terms of a weakly coupled classical gravity theory, which encodes information of the boundary theory.

In the prototypical example [22] of this duality, the gauge theory is $\mathcal{N}=4$ supersymmetric $S U\left(N_{C}\right)$ Yang-Mills theory (SYM), the maximally supersymmetric gauge field theory in four-dimensional space-time. The gravitational dual is Type IIB supergravity or string theory [29] ${ }^{1}$, depending on the gauge coupling and the number of colors $N_{C}$, in a direct product of five-dimensional AdS space-time and a five-sphere: $\operatorname{AdS}_{5} \times S^{5}$. If $g$ is the gauge coupling of the Yang-Mills theory, then in the limit $N_{C} \rightarrow \infty$, with $g^{2} N_{C} \gg 1$ but finite, the limit of large 't Hooft coupling, $g^{2} N_{C}$, ensures that the spacetime geometry has curvature $\mathcal{R}$ much smaller than the string scale $1 / l_{s}^{2}$ so that classical gravity is a good approximation. A small curvature $\mathcal{R}$, thus implies a large AdS radius $R, \mathcal{R} \sim 1 / R^{2}$, where $R=\left(4 \pi g^{2} N_{C}\right)^{1 / 4} l_{s}$ [22]. Since the gauge coupling $g$ and string coupling $g_{s}$ are related by $g^{2}=g_{s}$, the limit $N_{C} \rightarrow \infty$ ensures that the string coupling is small, so that stringy effects decouple ${ }^{2}$.

Anti-de Sitter $\operatorname{AdS}_{d+1}$-dimensional space-time is the maximally symmetric $d+1$ space with negative constant curvature and a $d$-dimensional flat space-time boundary. In Poincaré coordinates $x^{0}, x^{1}, \cdots, x^{d}, z \equiv x^{d+1}$, where the asymptotic border to the physical four-dimensional space-time is given by $z=0$, the line element is

$$
d s^{2}=\frac{R^{2}}{z^{2}}\left(\eta_{\mu \nu} d x^{\mu} d x^{\nu}-d z^{2}\right)
$$

where $\eta_{\mu \nu}$ is the usual Minkowski metric in $d$ dimensions. The most general group of transformations that leave the $\mathrm{AdS}_{d+1}$ differential line element invariant, the isometry group $S O(2, d)$ has dimension $(d+1)(d+2) / 2$. In the AdS/CFT correspondence, the consequence of the $S O(2,4)$ isometry of $\mathrm{AdS}_{5}$ is the conformal invariance of the dual field theory. Five-dimensional anti-de Sitter space $\mathrm{AdS}_{5}$ has 15 isometries, which induce in the Minkowski-space boundary theory the symmetry under the conformal group

\footnotetext{
${ }^{1} \mathrm{~A}$ brief discussion of holographic top-down duality with string theory is given in Chapter 7 .

${ }^{2} \mathrm{~A}$ recent review of large $N_{C}$ gauge theories is given in Ref. [30].
} 
$\operatorname{Conf}\left(R^{1,3}\right)$ with 15 generators in four dimensions: 6 Lorentz transformations plus 4 space-time translations plus 4 special conformal transformations plus 1 dilatation [31]. This conformal symmetry implies that there can be no scale in the theory and therefore also no discrete spectrum. Indeed, $\mathcal{N}=4$ supersymmetric $S U\left(N_{C}\right)$ Yang-Mills theory is a conformal field theory.

The AdS/CFT correspondence can be extended to non-conformal and supersymmetric or non-supersymmetric quantum field theories, a duality also known as "gauge/gravity" or "gauge/string" duality, which expresses well the generality of the conjectured duality. In particular, it is important to note that the conformal invariance of the prototypical example, $\mathcal{N}=4$ supersymmetric $S U(N)$ Yang-Mills theory in $3+1$ dimensions, is not required for the existence of a higher-dimensional gravity dual, and one can deform the original background geometry, giving rise to less symmetric gravity duals of confining theories with large 't Hooft coupling $g^{2} N_{C}[32,33]$. For example Polchinski and Strassler considered a modification of $\mathcal{N}=4$ Yang-Mills theory which includes $\mathcal{N}=1$ supersymmetry-preserving masses for some of the fields (the $\mathcal{N}=1$ chiral multiplets), and they describe the gravity dual of this theory in a certain limit of scales and 't Hooft coupling [32]. The nonvanishing masses break the conformal symmetry, and the resulting theory is confining at low energies. Another way to arrive at a non-conformal theory is to consider systems with nonvanishing temperature [34, 35, 36, 37], where one coordinate is compactified. Yet another example is the Sakai-Sugimoto (SS) model [38, 39], based on a specific brane construction in Type IIA string theory [29]; however since it is similar to finite temperature models, it is neither conformal nor supersymmetric. The SS model is notable in that it is confining and contains vector mesons and pions in its spectrum from the breaking of $S U\left(N_{f}\right) \times S U\left(N_{f}\right)$ chiral symmetry. We will describe this model in Chapter 7.

The AdS/CFT duality provides a useful guide in the attempt to model QCD as a higher-dimensional gravitational theory, but in contrast with the "top-down" holographic approach described above, which is to a great extent constrained by the symmetries, no gravity theory dual to QCD is known. The boundary (four-dimensional) quantum field theory, defined at the asymptotic AdS boundary at $z=0$, becomes the initial state of the higher-dimensional gravity theory (the bulk theory). However, to construct a dual holographic theory starting from a given quantum field theory in physical flat space-time, one would require in addition to the boundary conditions - the boundary theory, precise knowledge of the dynamical evolution in the bulk. Therefore, for phenomenological purposes it is more promising to follow a "bottom-up" approach, 
that is to start from a realistic 4-dimensional quantum field theory and look for a corresponding higher dimensional classical gravitational theory which encodes basic aspects of the boundary theory.

QCD is fundamentally different from the supersymmetric Yang-Mills theory occurring in the Maldacena correspondence. In contrast with QCD, where quarks transform under the fundamental representation of $S U(3)$, in SYM all quark fields transform under the adjoint representations of $S U\left(N_{C}\right)$. The conformal invariance of SYM theories implies that the $\beta$-function vanishes and, therefore, the coupling is scale independent. On the AdS side, the conformal symmetry corresponds to the maximal symmetry of this space. The classical QCD Lagrangian with massless quarks is also conformally invariant in four dimensions where its coupling $g_{s}$ is dimensionless. A scale, however, is introduced by quantum effects, and therefore its conformal invariance is broken and its coupling depends on the energy scale $\mu$ at which it is measured. We may compute the scale at which $g_{s}^{2}(\mu) / 4 \pi$ becomes of order 1 , as we follow the evolution of the coupling from high energy scales. This roughly defines the scale $\Lambda_{\mathrm{QCD}}$ which signals the transition from the perturbative region with quark and gluon degrees of freedom to the nonperturbative regime where hadrons should emerge. This mechanism is know as 'dimensional transmutation', whereby the conformal symmetry of the classical theory is anomalously broken by quantization, thus introducing a dimensionfull parameter, the mass scale $\Lambda_{\mathrm{QCD}}$.

QCD is asymptotically free [40,41], so at high energies it resembles a rather simple scale invariant theory. This is in fact one important argument for the relevance of anti-de Sitter space in applications of the AdS/CFT correspondence to QCD. For high energies or small distances the small coupling $g_{s}$ allows one to compute the corrections to scale invariance. This is certainly not the case in the infrared regime (IR), for distances comparable to the hadronic size, where perturbation theory breaks down. There is however evidence from lattice gauge theory [42], Dyson Schwinger equations [43, 44], and empirical effective charges [45], that the QCD $\beta$-function vanishes in the infrared. In a confining theory where the gluons have an effective mass or maximal wavelength, all vacuum polarization corrections to the gluon self-energy should decouple at long wavelengths [9]. Thus, from a physical perspective an infrared fixed point appears to be a natural consequence of confinement [46]. In fact, the running of the QCD coupling in the infrared region for $Q^{2}<4 \lambda$, where $\sqrt{\lambda}$ represents the hadronic mass scale, is expected to have the form $\alpha_{s}\left(Q^{2}\right) \propto \exp \left(-Q^{2} / 4 \lambda\right)$ [47], which agrees with the shape of the effective charge defined from the Bjorken sum rule, displaying an infrared fixed point. In the nonperturbative domain soft gluons are in effect sublimated into the effective con- 
fining potential. Above this region, hard-gluon exchange becomes important, leading to asymptotic freedom. The scale $\Lambda$ entering the evolution of the perturbative QCD running constant in a given renormalization scheme, such as $\Lambda_{\overline{M S}}$, can be determined in terms of the primary scheme-independent scale $\sqrt{\lambda}[48]$. This result is consistent with the hadronic flux-tube model [49] where soft gluons interact so strongly that they are sublimated into a color confinement potential for quarks. It is also consistent with the lack of empirical evidence confirming constituent gluons at small virtualities [50, 51]. At higher energy scales, $Q^{2}>4 \lambda$ we expect the usual perturbative QCD (PQCD) logarithmic dependence in $\alpha_{s}$ from the appearance of dynamical gluon degrees of freedom.

The relation between the dilatation symmetry and the symmetries in $\mathrm{AdS}_{5}$ can be seen directly from the AdS metric. The line element (1.1) is invariant under a dilatation of all coordinates. Since a dilatation of the Minkowski coordinates $x^{\mu} \rightarrow \rho x^{\mu}$ is compensated by a dilatation of the holographic variable $z \rightarrow \rho z$, it follows that the variable $z$ acts like a scaling variable in Minkowski space: different values of $z$ correspond to different energy scales at which a measurement is made. As a result, short spaceand time-like intervals map to the boundary in AdS space-time near $z=0{ }^{3}$. This corresponds to the ultraviolet (UV) region of AdS space.

A large four-dimensional interval of confinement dimensions $x_{\mu} x^{\mu} \sim 1 / \Lambda_{\mathrm{QCD}}^{2}$ maps to the large infrared region of AdS space $z \sim 1 / \Lambda_{\mathrm{QCD}}$. In order to incorporate the mechanisms of confinement in the gravity dual the conformal invariance encoded in the isometries of $\mathrm{AdS}_{5}$ must be broken. In bottom-up models the breaking of conformal symmetry is introduced by modifying the background AdS space-time at an infrared region of the geometries which sets the scale of the strong interactions. In this effective approach, one considers the propagation of hadronic modes in a fixed effective gravitational background asymptotic to AdS space, thus encoding prominent properties for QCD, such as the ultraviolet conformal limit at the AdS boundary at $z \rightarrow 0$, as well as modifications of the AdS background geometry in the large $z$ infrared region to describe confinement.

On the other hand, in models based on string theory - top-down models, the spacetime geometry is dictated by the corresponding brane configuration and may be quite different from $\mathrm{AdS}_{5}[38,39,53]$. A comparison of the predictions of AdS/QCD models in various space-time backgrounds appears in Ref. [54]. The result of such a comparison is that, for a wide class of background space-time geometries, naive predictions based on

\footnotetext{
${ }^{3}$ As quark and gluons can only travel over short distances as compared to the confinement scale $\Lambda_{\mathrm{QCD}}^{-1}$, the space-time region for their propagation is adjacent to the light-cone [52].
} 
five-dimensional AdS models (ignoring quantum corrections) are the most accurate. One of the reasons for the phenomenological success of models based on the AdS geometry might be that they capture a conformal window in QCD at the hadronic scale [46].

A simple way to obtain confinement and discrete normalizable modes is to truncate AdS space with the introduction of a sharp cut-off in the infrared region of AdS space, as in the "hard-wall" model [55], where one considers a slice of AdS space, $0 \leq z \leq z_{0}$, and imposes boundary conditions on the fields at the IR border $z_{0} \sim 1 / \Lambda_{\mathrm{QCD}}$. As first shown by Polchinski and Strassler [55], the modified AdS space provides a derivation of dimensional counting rules $[56,57,58]$ in QCD for the leading power-law fall-off of hard scattering beyond the perturbative regime. The modified theory generates the point-like hard behavior expected from QCD, instead of the soft behavior characteristic of extended objects [55]. On AdS space the physical states are represented by normalizable modes $\Phi_{P}(x, z)=e^{i P \cdot x} \Phi(z)$, with plane waves along Minkowski coordinates $x^{\mu}$ to represent a physical free hadron with momentum $P^{\mu}$, and a wave function $\Phi(z)$ along the holographic coordinate $z$. The hadronic invariant mass $P_{\mu} P^{\mu}=M^{2}$ is found by solving the eigenvalue problem for the AdS wave function $\Phi(z)$. This simple model fails however to reproduce the observed linear Regge behavior of hadronic excitations in $M^{2}$, a feature which is typical to many holographic models [59, 60].

One can also introduce a "dilaton" background in the holographic coordinate to produce a smooth cutoff at large distances as in the "soft-wall" model [61] which explicitly breaks the maximal AdS symmetry. In this bottom-up approach to AdS gravity, an effective $z$-dependent curvature is introduced in the infrared region of AdS which leads to conformal symmetry breaking in QCD, but its form is left largely unspecified. One can impose from the onset a viable phenomenological confining structure to determine the effective IR modification of AdS space. For example, one can adjust the dilaton background to reproduce the correct linear and equidistant Regge behavior of the hadronic mass spectrum $M^{2}$ [61], a form supported by semiclassical arguments [62]. One can also consider models where the dilaton field is dynamically coupled to gravity $[63,64,65,66,67,68]$. In one approach to AdS/QCD [69, 70, 71], bulk fields are introduced to match the $S U(2)_{L} \times S U(2)_{R}$ chiral symmetries of QCD and its spontaneous breaking, but without explicit connection with the internal constituent structure of hadrons [72]. Instead, axial and vector currents become the primary entities as in effective chiral theory. Following this bottom-up approach only a limited number of operators is introduced, and consequently only a limited number of fields is required to construct phenomenologically viable five-dimensional gravity duals. 


\subsection{Light-front holographic QCD}

Light-front quantization is the ideal relativistic, frame independent framework to describe the internal constituent structure of hadrons. The simple structure of the light-front (LF) vacuum allows an unambiguous definition of the partonic content of a hadron in QCD and of hadronic light-front wave functions (LFWFs), the underlying link between large distance hadronic states and the constituent degrees of freedom at short distances. The QCD light-front Hamiltonian $H_{L F}$ is constructed from the QCD Lagrangian using the standard methods of quantum field theory [73]. The spectrum and light-front wave functions of relativistic bound states are obtained from the eigenvalue equation $H_{L F}|\psi\rangle=M^{2}|\psi\rangle$. It becomes an infinite set of coupled integral equations for the LF components $\psi_{n}=\langle n \mid \psi\rangle$ in a Fock-state expansion, $i$. e. in a complete basis of non-interacting $n$-particle states $|n\rangle$, with an infinite number of components. This provides a quantum-mechanical probabilistic interpretation of the structure of hadronic states in terms of their constituents at the same light-front time $x^{+}=x^{0}+x^{3}$, the time marked by the front of a light wave [21]. The constituent spin and orbital angular momentum properties of the hadrons are also encoded in the LFWFs. Unlike instant time quantization, the Hamiltonian eigenvalue equation in the light front is frame independent. In practice, the matrix diagonalization [73] of the LF Hamiltonian eigenvalue equation in four-dimensional space-time has proven to be a daunting task because of the large size of the matrix representations. Consequently, alternative methods and approximations are necessary to better understand the nature of relativistic bound states in the strong-coupling regime of QCD.

To a first semiclassical approximation, where quantum loops and quark masses are not included, the relativistic bound-state equation for light hadrons can be reduced to an effective LF Schrödinger equation by identifying as a key dynamical variable the invariant mass of the constituents, which is the measure of the off-shellness in the LF kinetic energy, and it is thus the natural variable to characterize the hadronic wave function. In conjugate position space, the relevant dynamical variable is an invariant impact kinematical variable $\zeta$, which measures the separation of the partons within the hadron at equal light-front time [74]. Thus, by properly identifying the key dynamical variable, one can reduce, to a first semi-classical approximation, the multiparton problem in QCD to an effective one dimensional quantum field theory. As a result, all the complexities of the strong interaction dynamics are hidden in an effective potential $U(\zeta)$, but the central question - how to derive the confining potential from QCD, remains open. 
It is remarkable that in the semiclassical approximation described above, the lightfront Hamiltonian has a structure which matches exactly the eigenvalue equations in AdS space. This offers the unique possibility to make an explicit connection of the AdS wave function $\Phi(z)$ with the internal constituent structure of hadrons. In fact, one can obtain the AdS wave equations by starting from the semiclassical approximation to light-front QCD in physical space-time - an emergent property of this framework. This connection yields a relation between the coordinate $z$ of AdS space with the impact LF variable $\zeta$ [74], thus giving the holographic variable $z$ a precise definition and intuitive meaning in light-front QCD.

Light-front holographic methods were originally introduced $[75,76]$ by matching the electromagnetic current matrix elements in AdS space [77] with the corresponding expression derived from light-front quantization in physical space-time [78, 79]. It was also shown that one obtains identical holographic mapping using the matrix elements of the energy-momentum tensor [81] by perturbing the AdS metric (1.1) around its static solution [82], thus establishing a precise relation between wave functions in AdS space and the light-front wave functions describing the internal structure of hadrons.

The description of higher spin in AdS space is a notoriously difficult problem [83, $84,85,86,87,88,89,90]$, and thus there is much interest in finding a simplified approach which can describe higher-spin hadrons using the gauge/gravity duality. In the framework of Ref. [61] the recurrences of the $\rho$ and its daughter trajectories are obtained from a gauge invariant AdS Lagrangian. In the light-front holographic approach, where the internal structure, and notably the orbital angular momentum of the constituents, is reflected in the AdS wave functions by the LF mapping, wave equations with arbitrary integer and half-integer spin can be derived from an invariant effective action in AdS space [91]. Remarkably, the pure AdS equations correspond to the light-front kinetic energy of the partons inside a hadron, whereas the light-front interactions which build confinement correspond to the modification of AdS space in an effective dual gravity approximation [74]. From this point of view, the non-trivial geometry of pure AdS space encodes the kinematical aspects and additional deformations of AdS space encode dynamics, including confinement, and determine the form of the effective potential $U$ from the precise holographic mapping to light-front physics. It can also be shown that the introduction of a dilaton profile is equivalent to a modification of the AdS metric, even for arbitrary spin [91].

It is important to notice that the construction of higher-spin modes given in Ref. [61] starts from a gauge invariant action in AdS and uses the gauge invariance of the model to 
construct a higher-spin effective action. However, this approach which is based in gauge invariance in the higher dimensional theory, is not applicable to light-front mapping to physical space-time which incorporates LF partonic physics in the holographic approach. In contrast, for light-front mapping the identification of orbital angular momentum of the constituents with the fifth dimensional AdS mass, in principle an arbitrary parameter, is a key element in the description of the internal structure of hadrons using light-front holographic principles, since hadron masses depend crucially on it.

\subsection{Confinement and conformal algebraic structures}

In principle, LF Hamiltonian theory provides a rigorous, relativistic and frameindependent framework for solving nonperturbative QCD and understanding the central problem of hadron physics - color confinement. For QCD $(1+1)$ the mass of the mesons and baryon eigenstates at zero quark mass is determined in units of its dimensionful coupling using the Discretized Light Cone Quantization (DLCQ) method [92, 93]. However, in the case of $3+1$ space-time, the QCD coupling is dimensionless, so the physical mechanism that sets the hadron mass scale for zero quark mass is not apparent. Since our light-front semiclassical approximation [73] is effectively a one-dimensional quantum field theory, it is natural to apply the framework developed by de Alfaro, Fubini and Furlan (dAFF) [94] which can generate a mass scale and a confinement potential without affecting the conformal invariance of the action. In their remarkable paper, published some 40 years ago, a hint to the possible appearance of scale in nominally conformal theories was given [94]. This remarkable result is based on the isomorphism of the algebra of the one-dimensional conformal group $\operatorname{Conf}\left(R^{1}\right)$ to the algebra of generators of the group $S O(2,1)$ and the isometries of $\mathrm{AdS}_{2}$ space. In fact, one of the generators of this group, the rotation in the 2-dimensional space, is compact and has therefore a discrete spectrum with normalizable eigenfunctions. As a result, the form of the evolution operator is fixed and includes a confining harmonic oscillator potential, and the time variable has a finite range. Since the generators of the conformal group have different dimensions their relations with generators of $S O(2,1)$ imply a scale, which here plays a fundamental role, as already conjectured in [94]. These considerations have led to the realization that the form of the effective LF confining potential can be obtained by extending the results found by dAFF to light-front dynamics and to the embedding 
space $[95]^{4}$. These results become particularly relevant, since it was also shown recently that an effective harmonic potential in the light-front form of dynamics corresponds, for light quark masses, to a linear potential in the usual instant-form [97, 98]. Thus, these results also lead to the prediction of linear Regge trajectories in the hadron mass square for small quark masses in agreement with the observed spectrum for light hadrons.

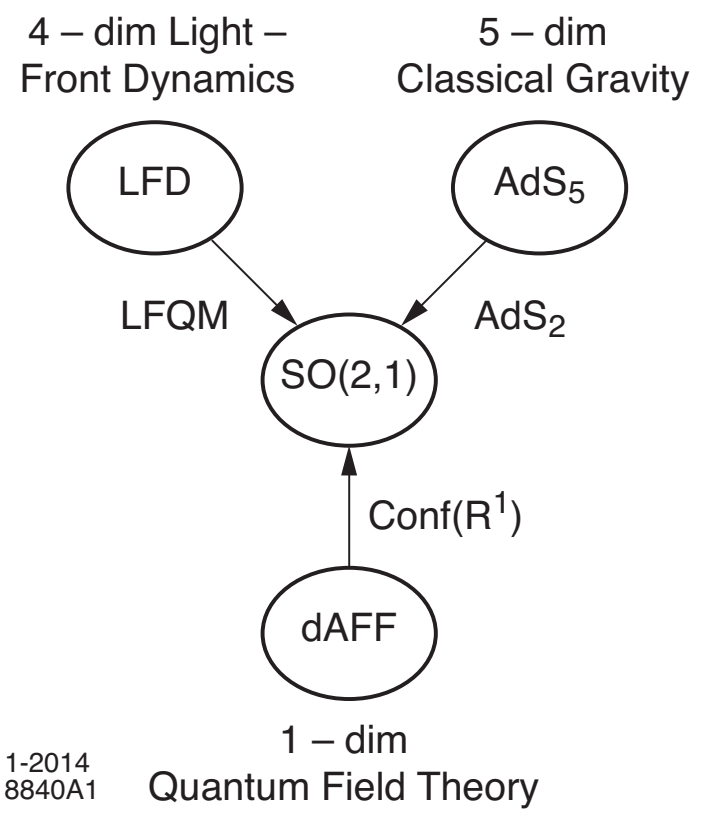

Figure 1.1: An effective light-front theory for QCD endowed with an $S O(2,1)$ algebraic structure follows from the one-dimensional semiclassical approximation to light-front dynamics in physical space-time, higher dimensional gravity in $\mathrm{AdS}_{5}$ space and the extension of conformal quantum mechanics to light-front dynamics. The result is a relativistic light-front quantum mechanical wave equation which incorporates essential spectroscopic and dynamical features of hadron physics. The emergence of a mass scale and the effective confining potential has its origins in the isomorphism of the one-dimensional conformal group Conf $\left(R^{1}\right)$ with the group $S O(2,1)$, which is also the isometry group of $\mathrm{AdS}_{2}$.

The remarkable connection between the semiclassical approximation to light-front dynamics in physical four-dimensional space-time with gravity in a higher dimensional AdS space, and the constraints imposed by the invariance properties under the full conformal group in one dimensional quantum field theory, is depicted in Fig. 1.1 and is central to this report. We shall describe how to construct a light-front effective theory which encodes the fundamental conformal symmetry of the four-dimensional classical

\footnotetext{
${ }^{4}$ Harmonic confinement also follows from the covariant Hamiltonian description of mesons given in Ref. [96].
} 
QCD Lagrangian. This construction is endowed with and $S O(2,1)$ underlying symmetry, consistent with the emergence of a mass scale. We will also describe how to obtain effective wave equations for any spin in the higher dimensional embedding space, and how to map these results to light-front physics in physical space-time. The end result is a semiclassical relativistic light-front bound-state equation, similar to the Schrödinger equation in atomic physics, which describes essential spectroscopic and dynamical features of hadron physics.

\subsection{Other approaches and applications}

A completely different approach to an effective treatment of nonperturbative QCD, which, however turns out to be closely related to holographic QCD is a "meromorphization procedure" of perturbative QCD. In fact, it has been shown [99] that the hard wall model corresponds to a procedure proposed by Migdal [100, 101], whereas the soft wall model has been related to the QCD sum rule method [102] in Refs. [103, 104] (See also Appendix F). Other approaches to emergent holography are discussed in $[105,106,107,108,109,110,111,112]$.

We also briefly review other holographic approaches to QCD, in particular top-down and bottom-up models based on chiral symmetry breaking. Top-down models, such as the Sakai-Sugimoto model, are derived from brane configurations in string theory, whereas bottom-up models, such as the hard or soft-wall models, are more phenomenological and are not derived from string theory. Each of the models discussed in this review include degrees of freedom which are identified with Standard Model hadrons via their quantum numbers, and predictions of holographic models for QCD observables may be compared to experiment and to other models, often with remarkable quantitative success $[113,114]$. The domain of small coupling in QCD would require, however, quantum corrections beyond the semiclassical approximation [115].

A particularly interesting application of the holographic ideas is to high-energy smallangle scattering in QCD, usually described by pomeron exchange [116] which carries the vacuum quantum numbers. The gauge/string duality provides a unified framework for the description of the soft Regge regime and hard BFKL Pomeron [117]. The gauge/string framework can also be used to compute strong coupling high-energy oderon exchange [118], which distinguish particle anti-particle cross sections and thus carries $C=-1$ vacuum quantum numbers. The gauge/gravity duality has also been applied to deep inelastic scattering (DIS), first discussed in this context in Ref. [77]. We will 
not discuss in this report these interesting applications, but refer the reader to the original articles cited here ${ }^{5}$. Neither shall we discuss in this report applications of the gauge/gravity duality to strongly coupled quark-gluon plasma observed in heavy ion collisions at RHIC and CERN, also an important subject which has attracted much attention ${ }^{6}$.

Another interesting topic which we only touch upon in this report is holographic renormalization [127]: the relation between the flow in the holographic coordinate in AdS space and the renormalization group flow of the dual quantum field theory [128] ${ }^{7}$. Thus, the description of the large-scale behavior should be independent of the of the "microscopic" degrees of freedom (quarks and gluons) of the ultraviolet boundary theory and expressed in terms of "macroscopic" infrared degrees of freedom (hadrons). As a result, the interaction potential of the effective infrared theory should retain universal characteristics from the renormalization group flow. For example in hadronic physics the universality of the Regge trajectories, but this universal behavior should also be relevant to other areas.

A number of excellent reviews on the AdS/CFT correspondence are already available. We refer the reader to the Physics Report by Aharony, et al. [131], the TASI lectures by Klebanov [132] and by D'Hoker and Freedman [133] for some early reviews. For more recent discussions of holographic QCD see the reviews in Refs. [134, 135, 136, 137].

\subsection{Contents of this review}

The report is organized as follows: in Chapter 2 we describe important aspects of light-front quantization and its multi-parton semiclassical approximation. This leads to a relativistic invariant light-front wave equation to compute hadronic bound states in terms of an effective potential which is a priori unknown. We also discuss how the semiclassical results are modified by the introduction of light quark masses. We show in Chapter 3 how a specific introduction of a scale determines uniquely the form of the light-front effective confinement potential, while leaving the action conformally invariant. We also describe in this chapter the relation of the one-dimensional conformal group with the group $S O(2,1)$, and the extension of conformal quantum mechanics to

\footnotetext{
${ }^{5}$ Other interesting applications of the gauge/gravity correspondence include, but are not limited to, high-energy $p p$ and $p \bar{p}$ scattering [119, 120], high-energy photon-hadron scattering [121], compton scattering [122, 123, 124] and polarized DIS [125].

${ }^{6}$ For a review see Ref. [126] and references therein.

${ }^{7}$ For a review of holographic renormalization, see for example [129, 130]
} 
light-front physics described in Chapter 2. In Chapter 4 we derive hadronic AdS wave equations for arbitrary integer and half-integer spin. We give particular care to the separation of kinematic and dynamical effects in view of the mapping to LF bound-state equations. We perform the actual light-front mapping in Chapter 5, and and we compare the theoretical results with the observed light meson and baryon spectra. In Chapter 6 we carry out the actual LF mapping of amplitudes in AdS to their corresponding expressions in light-front QCD. We describe form factors and transition amplitudes of hadrons in holographic QCD. We also give a comparison with data and we discuss present limitations of the model. In Chapter 7 we present other approaches to holographic QCD, including bottom-up and top-down gauge/gravity models. We present our conclusions and final remarks in Chapter 8. We include a discussion of open problems and future applications. In particular, we point out a possible connection of our effective light-front approach with holographic renormalization flows to $\mathrm{AdS}_{2}$ geometry in the infrared and its one-dimensional conformal dual theory. In Appendix A we give a brief introduction to Riemanian geometry and maximally symmetric Riemannian spaces. In particular we exhibit the connection between the conformal group in one dimension, $S O(2,1)$, and $\mathrm{AdS}_{2}$. In Appendix B we give a short collection of notations and conventions. We present in the Appendices $\mathrm{C}$ and $\mathrm{D}$ several more technical derivations, relevant for Chapter 3 and 4 respectively. We describe in Appendix E the light-front holographic mapping of the gravitational form factor of composite hadrons. In Appendix F we discuss the relation of the generating functional of the boundary conformal field theory and the classical action in the 5-dimensional gravity theory $[27,28]$ for fields with arbitrary integer spin, both in the soft- and the hard-wall models. In Appendix G some useful formulæ are listed. In Appendix $\mathrm{H}$ we describe an algebraic procedure to construct the holographic light-front Hamiltonians corresponding to the hard and soft-wall models discussed in this report for bosons and fermions [138]. Finally in Appendix I we describe the equations of motion of $p$-form fields in AdS. 


\section{Chapter 2}

\section{A Semiclassical Approximation to Light-Front Quantized QCD}

Light-front quantization is the natural framework for the description of the QCD nonperturbative relativistic bound-state structure in quantum field theory in terms of a frame-independent $n$-particle Fock expansion. The central idea is due to Dirac who demonstrated the remarkable advantages of using light-front time $x^{+}=x^{0}+x^{3}$ (the "front-form") to quantize a theory versus the standard time $x^{0}$ (the "instant-form"). As Dirac showed [21], the front-form has the maximum number of kinematic generators of the Lorentz group, including the boost operator. Thus the description of a hadron at fixed $x^{+}$is independent of the observer's frame, making it ideal for addressing dynamical processes in quantum chromodynamics. An extensive review of light-front quantization is given in Ref. [73]. As we shall discuss in this and in the next two chapters, a semiclassical approximation to light-front quantized field theory in physical four-dimensional space-time has a holographic dual with dynamics of theories in five-dimensional antide Sitter space. Furthermore, its confining dynamics follows from the mapping to a one-dimensional conformal quantum field theory [95].

Quantization in the light-front provides a rigorous field-theoretical realization of the intuitive ideas of the parton model $[139,140]$ formulated at fixed time $x^{0}$ in the infinite-momentum frame [141, 142]. Historically, the prediction of Bjorken scaling in deep inelastic scattering [143] followed from a combination of the high energy limit $q_{0} \rightarrow i \infty$ with the infinite momentum frame $P \rightarrow \infty$, introduced in [141], using the usual definition of time; i.e., the instant-form. The same results are obtained in the front-form but with complete rigor; e.g., the structure functions and other probabilistic parton distributions measured in deep inelastic scattering are obtained from the squares 
of the light-front wave functions, the eigensolution of the light-front Hamiltonian. Unlike the instant-form, the front-form results are independent of the hadron's Lorentz frame. A measurement in the front form is analogous to taking a flash photograph. The image in the resulting picture records the state of the object as the front of a light wave from the flash illuminates it, consistent with observations within the space-like causal horizon $\Delta x_{\mu}^{2}<0$. Similarly, measurements such as deep inelastic electron-proton scattering, determine the structure of the target proton at fixed light-front time.

In the constituent quark model $[144,145]$ the minimum quark content required by the hadronic quantum numbers is included in the wave functions, which describe how hadrons are built of their constituents. In the conventional interpretation of the quark model, the main contribution to the hadron masses is supposed to arise from the explicit breaking of chiral symmetry by constituent quark masses. Typical computations of the hadron spectrum generally include a spin-independent confining interaction and a spindependent interaction, usually modeled from one-gluon-exchange in QCD [146]. The parton model and the constituent quark model provide, respectively, a good intuitive understanding of many high- and low-energy phenomena. In practice, however, it has been proven difficult to reconcile the constituent quark model with QCD, and the best hope to make a connection between both approaches is provided by light-front dynamics. In fact, the original formulation of QCD was given in light-front coordinates [147, 148] and the idea to derive a light-front constituent quark model [149] also dates to the same time. The physical connections of the front-form with the constituent model is a reason to hope that light-front quantization will eventually provide an understanding of the most challenging dynamical problems in QCD, such as color confinement [150].

Just as in non-relativistic quantum mechanics, one can obtain bound-state light-front wave functions in terms of the hadronic constituents from solving the light-front Hamiltonian eigenvalue problem. The eigenstates of the light-front Hamiltonian are defined at fixed light-front time $x^{+}$over all space within the causal horizon, so that causality is maintained without normal-ordering. In fact, light-front physics is a fully relativistic field theory but its structure is similar to non-relativistic theory [21], and the bound-state equations are relativistic Schrödinger-like equations at equal light-front time. Because of Wick's theorem, light-front time-ordered perturbation theory is equivalent to the covariant Feynman perturbation theory. Furthermore, since boosts are kinematical, the light-front wave functions are frame independent.

In principle, one can solve QCD by diagonalizing the light-front QCD Hamiltonian $H_{L F}$ using, for example, the discretized light-cone quantization method [73] or 
the Hamiltonian transverse lattice formulation introduced in [151]. The spectrum and light-front wave functions are then obtained from the eigenvalues and eigenfunctions of the Heisenberg problem $H_{L F}|\psi\rangle=M^{2}|\psi\rangle$, which becomes an infinite set of coupled integral equations for the light-front components $\psi_{n}=\langle n \mid \psi\rangle$ in a Fock expansion [73]. This nonperturbative method has the advantage that it is frame-independent, is defined in physical Minkowski space-time, and has no fermion-doubling problem. It has been applied successfully in lower space-time dimensions [73], such as $\mathrm{QCD}(1+1)$ [92, 93]. In practice, solving the actual eigenvalue problem is a formidable computational task for a non-abelian quantum field theory in four-dimensional space-time. An analytic approach to nonperturbative relativistic bound-states is also vastly difficult because of the unbound particle number with arbitrary momenta and helicities. Consequently, alternative methods and approximations are necessary to better understand the nature of relativistic bound-states in the strong-coupling regime.

Hadronic matrix elements and form factors are computed from simple overlaps of the boost invariant light-front wave functions as in the Drell-Yan West formula [78, 79]. In contrast, at ordinary fixed time $x^{0}$, the hadronic states must be boosted from the hadron's rest frame to a moving frame - an intractable dynamical problem which involves changes in particle number. Moreover, the form factors at fixed time $x^{0}$ also require computing off-diagonal matrix elements and the contributions of currents which arise from the instant vacuum fluctuations in the initial state and which connect to the hadron in the final state. Thus, the knowledge of wave functions alone is not sufficient to compute covariant current matrix elements in the usual instant form. When a hadron is examined in the light front in the Drell-Yan frame [78, 80], for example, a virtual photon couples only to forward moving quarks and only processes with the same number of initial and final partons are allowed. A quantum-mechanical probabilistic constituent interpretation in terms of wave functions is thus an important property of light-front dynamics required for both the constituent quark model and the parton model.

In axiomatic quantum field theory the vacuum state is defined as the unique state invariant under Poincaré transformations [152]. Conventionally it is defined as the lowest energy eigenstate of the instant-form Hamiltonian. Such an eigenstate is defined at a single time $x^{0}$ over all space $x$. It is thus acausal and frame-dependent. In contrast, in the front form, the vacuum state is defined as the eigenstate of lowest invariant mass $M^{2}$ at fixed light-front time $x^{+}=x^{0}+x^{3}$. It is frame-independent and only requires information within the causal horizon. Thus, an important advantage of the LF framework is the apparent simplicity and Lorentz invariance of the LF vacuum. In 
contrast, the equal-time vacuum contains quantum loop graphs and thus an infinite sea of quarks and gluons.

\subsection{The Dirac forms of relativistic dynamics}

According to Dirac's classification of the forms of relativistic dynamics [21], the fundamental generators of the Poincaré group can be separated into kinematical and dynamical generators. The kinematical generators act along the initial hypersurface where the initial conditions (the quantization conditions) are imposed. The kinematical generators leave invariant the initial surface and are thus independent of the dynamics; therefore they contain no interactions. The dynamical generators are responsible for the evolution of the system (mapping one initial surface into another surface) and depend consequently on the interactions.
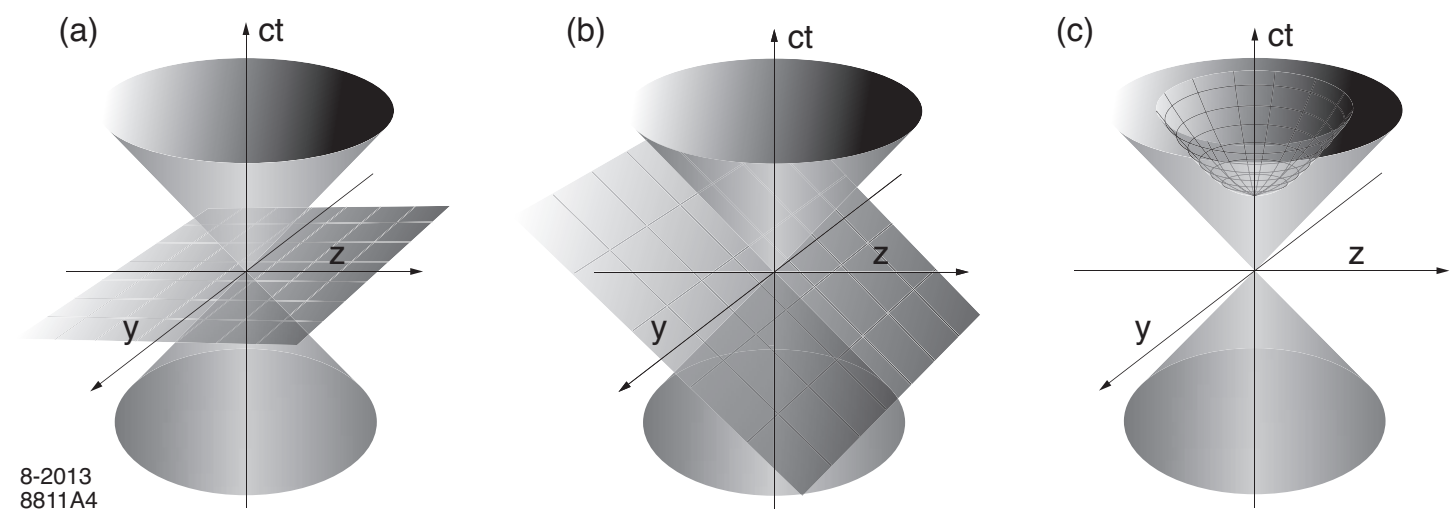

Figure 2.1: Dirac forms of relativistic dynamics: (a) the instant form, (b) the front form and (c) the point form. The initial surfaces are defined respectively by $x^{0}=0, x^{0}+x^{3}=0$ and $x^{2}=\kappa^{2}>0, x^{0}>0$.

In his original paper Dirac [21] found three forms of relativistic dynamics ${ }^{1}$ which correspond to different parameterizations of space-time and which cannot be transformed into each other by a Lorentz transformation. The three forms of Dirac are illustrated in Fig. 2.1. The instant form is the usual form where the initial surface is the surface defined at $x^{0}=0$. In the front form (discussed above) the initial surface is the tangent plane to the light-cone $x^{0}+x^{3}=0$ - the null plane, thus without reference to a specific Lorentz frame. According to Dirac [21], it is the "three dimensional surface in space-time

\footnotetext{
${ }^{1}$ Subsequently Leutwyler and Stern [153] found two additional forms for a total of five inequivalent forms which correspond to the number of subgroups of the Poincaré group.
} 
formed by a plane wave front advancing with the velocity of light." In the third form, the point form, the initial surface is the hyperboloid defined by $x^{2}=\kappa^{2}>0, x^{0}>0$, which is left invariant by the Lorentz generators. Each front has its own Hamiltonian and evolves with a different time, but the results computed in any front should be identical, since physical observables cannot depend on how space-time is parameterized.

The Poincaré group is the full symmetry group of any form of relativistic dynamics. Its Lie algebra is given by the well known commutation relations

$$
\begin{aligned}
{\left[P^{\mu}, P^{\nu}\right] } & =0 \\
{\left[M^{\mu \nu}, P^{\rho}\right] } & =i\left(g^{\mu \rho} P^{\nu}-g^{\nu \rho} P^{\mu}\right), \\
{\left[M^{\mu \nu}, M^{\rho \sigma}\right] } & =i\left(g^{\mu \rho} M^{\nu \sigma}-g^{\mu \sigma} M^{\nu \rho}+g^{\nu \sigma} M^{\mu \rho}-g^{\nu \rho} M^{\mu \sigma}\right),
\end{aligned}
$$

where the $P^{\mu}$ are the generators of space-time translations and the antisymmetric tensor $M^{\mu \nu}$ of the generators of the Lorentz transformations.

In the instant form the Hamiltonian $P^{0}$ and the three components of the boost vector $K^{i}=M^{0 i}$ are dynamical generators, whereas the momentum $\mathbf{P}$ and the three components of angular momentum $J^{i}=\frac{1}{2} \epsilon^{i j k} M^{j k}$ are kinematical. In the front form [154, 155], the dynamical generators are the "minus" component generators, the Hamiltonian $P^{-}$and the generator $M^{-1}=K^{1}-J^{2}$ and $M^{-2}=K^{2}+J^{1}$, which correspond to LF rotations along the $x$ and $y$-axes ${ }^{2}$. The kinematical generators are the longitudinal "plus" momentum $P^{+}$and transverse momentum $P^{i}$. The boost operators are also kinematical in the light-front: $M^{+1}=K^{1}+J^{2}$ and $M^{+2}=K^{2}-J^{1}$, which boost the system in the $x$ - and $y$-direction respectively, as well as the generator $\frac{1}{2} M^{+-}=K^{3}$ which boost the system in the longitudinal direction. Finally, the $z$-component of angular momentum $M^{12}=J^{3}$, which rotates the system in the $x-y$ plane is also a kinematical operator, and labels the angular moment states in the light front. In the point-form the four generators $P^{\mu}$ are dynamical and the six Lorentz generators $M^{\mu \nu}$ kinematical. The light-front frame has the maximal number of kinematical generators.

\subsection{Light-front dynamics}

For a hadron with four momentum $P^{\mu}=\left(P^{+}, P^{-}, \mathbf{P}_{\perp}\right), P^{ \pm}=P^{0} \pm P^{3}$, the massshell relation $P^{2}=M^{2}$, where $P_{\mu} P^{\mu}=P^{+} P^{-}-\mathbf{P}_{\perp}^{2}$, leads to the dispersion relation for

\footnotetext{
${ }^{2}$ The \pm components of a tensor are defined by $a^{ \pm}=a^{0} \pm a^{3}$, and the metric follows from the scalar product $a \cdot b=\frac{1}{2}\left(a^{+} b^{-}+a^{-} b^{+}\right)-a^{1} b^{1}-a^{2} b^{2}$ (See Appendix B).
} 
the LF Hamiltonian $P^{-}$

$$
P^{-}=\frac{\mathbf{P}_{\perp}^{2}+M^{2}}{P^{+}}, \quad P^{+}>0
$$

This LF relativistic dispersion relation has several remarkable properties. The square root operator does not appear in (2.2), and thus the dependence on the transverse momentum $\mathbf{P}_{\perp}$ is similar to the non-relativistic dispersion relation. For massive physical hadronic states $P^{2}>0$ and $P^{0}$ are positive, thus $P^{+}$and $P^{-}$are also positive. Furthermore, since the longitudinal momentum $P^{+}$is kinematical, it is given by the sum of the single-particle longitudinal momentum of the constituents of the bound state. In fact, for an $n$-particle bound state with particle four momentum $p_{i}^{\mu}=\left(p_{i}^{+}, p_{i}^{-}, \mathbf{p}_{\perp i}\right)$, where $p_{i}^{2}=p_{i}^{+} p_{i}^{-}-\mathbf{p}_{\perp i}^{2}=m_{i}^{2}$, we have

$$
p_{i}^{-}=\frac{\mathbf{p}_{\perp i}^{2}+m_{i}^{2}}{p_{i}^{+}}, \quad p_{i}^{+}>0,
$$

for each constituent $i$. Thus

$$
P^{+}=\sum_{i}^{n} p_{i}^{+}, \quad p_{i}^{+}>0 .
$$

On the other hand, since the bound-state is arbitrarily off the LF energy shell we have the inequality

$$
P^{-}-\sum_{i}^{n} p_{i}^{-}<0,
$$

for the LF Hamiltonian which contains the interactions.

The LF Hamiltonian $P^{-}$is the momentum conjugate to the LF-time coordinate, $x^{+}=x^{0}+x^{3}$. Thus, the evolution of the system is given by the relativistic light-front Schrödinger-like equation

$$
i \frac{\partial}{\partial x^{+}}|\psi(P)\rangle=P^{-}|\psi(P)\rangle
$$

where $P^{-}$is given by (2.2). Since the generators $P^{+}$and $\mathbf{P}_{\perp}$ are kinematical, we can construct the LF Lorentz-invariant Hamiltonian $H_{L F}=P^{2}=P^{+} P^{-}-\mathbf{P}_{\perp}^{2}$ with eigenvalues corresponding to the invariant mass $P_{\mu} P^{\mu}=M^{2}$

$$
H_{L F}|\psi(P)\rangle=M^{2}|\psi(P)\rangle
$$

As one could expect, the eigenstates of the LF Hamiltonian $H_{L F}$ are invariant since the LF boost generators are kinematical. Consequently, if the eigenstates are projected onto 
an $n$-particle Fock component $|n\rangle$ of the free LF Hamiltonian, the resulting light-front wave function $\psi_{n}=\langle n \mid \psi\rangle$ only depends on the relative coordinates of the constituents. Thus, an additional important property of the light-front frame for a bound state is the separation of relative and overall variables.

Since $p_{i}^{+}>0$ for every particle, the vacuum is the unique state with $P^{+}=0$ and contains no particles. All other states have $P^{+}>0$. Since plus momentum is kinematic, and thus conserved at every vertex, loop graphs with constituents with positive $p_{i}^{+}$cannot occur in the light-front vacuum. Because this also holds in presence of interactions, the vacuum of the interacting theory is also the trivial vacuum of the non-interacting theory. However, one cannot discard the presence of zero modes, possible background fields with with $p^{+}=0$, which also lead to $P^{+}=0$ and thus can mix with the trivial vacuum. The light-front vacuum is defined at fixed LF time $x^{+}=x^{0}+x^{3}$ over all $x^{-}=x^{0}-x^{3}$ and $\mathbf{x}_{\perp}$, the expanse of space that can be observed within the speed of light. Thus the frame independent definition of the vacuum

$$
P^{2}|0\rangle=0
$$

Causality is maintained since the LF vacuum only requires information within the causal horizon. Since the LF vacuum is causal and frame independent, it can provide a representation of the empty universe for quantum field theory $[156,157]$. In fact, the front form is a natural basis for cosmology because the universe is observed along the front of a light wave.

\subsection{Light-front quantization of QCD}

We can now proceed to relate the LF generators to the underlying QCD Lagrangian in terms of the dynamical fields of the theory. In the light-front, the Dirac equation is written as a pair of coupled equations for plus and minus components, $\psi_{ \pm}=\Lambda_{ \pm} \psi$, with the projection operator $\Lambda_{ \pm}=\gamma^{0} \gamma^{ \pm}$. One of the equations does not have a derivative with respect to the $\mathrm{LF}$ evolution time $x^{+}$, and it is therefore a constraint equation which determines the minus component $\psi_{-}$in terms of the dynamical field $\psi_{+}[73,158]$. Likewise, the dynamical transverse field $\mathbf{A}_{\perp}$ in the light-cone gauge $A^{+}=0$ has no ghosts nor unphysical negative metric gluons. 
Our starting point is the $S U(3)_{C}$ invariant Lagrangian of QCD

$$
\mathcal{L}_{\mathrm{QCD}}=\bar{\psi}\left(i \gamma^{\mu} D_{\mu}-m\right) \psi-\frac{1}{4} G_{\mu \nu}^{a} G^{a \mu \nu}
$$

where $D_{\mu}=\partial_{\mu}-i g_{s} A_{\mu}^{a} T^{a}$ and $G_{\mu \nu}^{a}=\partial_{\mu} A_{\nu}^{a}-\partial_{\nu} A_{\mu}^{a}+g_{s} c^{a b c} A_{\mu}^{b} A_{\nu}^{c}$, with $\left[T^{a}, T^{b}\right]=i c^{a b c} T^{c}$ and $a, b, c$ are $S U(3)_{C}$ color indices.

One can express the hadron four-momentum generator $P^{\mu}=\left(P^{+}, P^{-}, \mathbf{P}_{\perp}\right)$ in terms of the dynamical fields $\psi_{+}$and $\mathbf{A}_{\perp}$ quantized on the light-front at fixed light-front time $x^{+}, x^{ \pm}=x^{0} \pm x^{3}[73]$

$$
\begin{aligned}
P^{-} & =\frac{1}{2} \int d x^{-} d^{2} \mathbf{x}_{\perp}\left(\bar{\psi}_{+} \gamma^{+} \frac{m^{2}+\left(i \nabla_{\perp}\right)^{2}}{i \partial^{+}} \psi_{+}-A^{a \mu}\left(i \nabla_{\perp}\right)^{2} A_{\mu}^{a}\right) \\
& +g_{s} \int d x^{-} d^{2} \mathbf{x}_{\perp} \bar{\psi}_{+} \gamma^{\mu} T^{a} \psi_{+} A_{\mu}^{a}+ \\
& +\frac{g_{s}^{2}}{4} \int d x^{-} d^{2} \mathbf{x}_{\perp} c^{a b c} c^{a d e} A_{\mu}^{b} A_{\nu}^{c} A^{d \mu} A^{e \nu} \\
& +\frac{g_{s}^{2}}{2} \int d x^{-} d^{2} \mathbf{x}_{\perp} \bar{\psi}_{+} \gamma^{+} T^{a} \psi_{+} \frac{1}{\left(i \partial^{+}\right)^{2}} \bar{\psi}_{+} \gamma^{+} T^{a} \psi_{+} \\
& +\frac{g_{s}^{2}}{2} \int d x^{-} d^{2} \mathbf{x}_{\perp} \bar{\psi}_{+} \gamma^{\mu} T^{a} A_{\mu}^{a} \frac{\gamma^{+}}{i \partial^{+}}\left(T^{b} A_{\nu}^{b} \gamma^{\nu} \psi_{+}\right),
\end{aligned}
$$

The first term in (2.10) is the kinetic energy of quarks and gluons; it is the only nonvanishing term in the limit $g_{s} \rightarrow 0$. The second term is the three-point vertex interaction. The third term is the four-point gluon interaction. The fourth term represents the instantaneous gluon interaction which originates from the imposition of light-cone gauge, and the last term is the instantaneous fermion interaction [73]. The integrals in (2.10) are over the null plane $x^{+}=0$, the initial surface, where the commutation relations for the fields are fixed. The LF Hamiltonian $P^{-}$generates LF time translations

$$
\left[\psi_{+}(x), P^{-}\right]=i \frac{\partial}{\partial x^{+}} \psi_{+}(x), \quad\left[\mathbf{A}_{\perp}, P^{-}\right]=i \frac{\partial}{\partial x^{+}} \mathbf{A}_{\perp}(x),
$$

which evolve the initial conditions for the fields to all space-time.

The light-front longitudinal momentum $\mathbf{P}^{+}$

$$
P^{+}=\int d x^{-} d^{2} \mathbf{x}_{\perp}\left(\bar{\psi}_{+} \gamma^{+} i \partial^{+} \psi_{+}-A^{a \mu}\left(i \partial^{+}\right)^{2} A_{\mu}^{a}\right)
$$


and the light-front transverse momentum $\mathbf{P}_{\perp}$

$$
\mathbf{P}_{\perp}=\frac{1}{2} \int d x^{-} d^{2} \mathbf{x}_{\perp}\left(\bar{\psi}_{+} \gamma^{+} i \nabla_{\perp} \psi_{+}-A^{a \mu} i \partial^{+} i \nabla_{\perp} A_{\mu}^{a}\right)
$$

are kinematical generators and do not involve interactions.

The Dirac field $\psi_{+}$and the transverse gluon field $\mathbf{A}_{\perp}$ are expanded in terms of particle creation and annihilation operators as [73]

$$
\psi_{+}\left(x^{-}, \mathbf{x}_{\perp}\right)_{\alpha}=\sum_{\lambda} \int_{q^{+}>0} \frac{d q^{+}}{\sqrt{2 q^{+}}} \frac{d^{2} \mathbf{q}_{\perp}}{(2 \pi)^{3}}\left[b_{\lambda}(q) u_{\alpha}(q, \lambda) e^{-i q \cdot x}+d_{\lambda}(q)^{\dagger} v_{\alpha}(q, \lambda) e^{i q \cdot x}\right]
$$

and

$$
\mathbf{A}_{\perp}\left(x^{-}, \mathbf{x}_{\perp}\right)=\sum_{\lambda} \int_{q^{+}>0} \frac{d q^{+}}{\sqrt{2 q^{+}}} \frac{d^{2} \mathbf{q}_{\perp}}{(2 \pi)^{3}}\left[a(q) \vec{\epsilon}_{\perp}(q, \lambda) e^{-i q \cdot x}+a(q)^{\dagger} \vec{\epsilon}_{\perp}^{*}(q, \lambda) e^{i q \cdot x}\right]
$$

with $u$ and $v$ LF spinors [159] and commutation relations

$$
\begin{gathered}
\left\{b(q), b^{\dagger}\left(q^{\prime}\right)\right\}=\left\{d(q), d^{\dagger}\left(q^{\prime}\right)\right\}=(2 \pi)^{3} \delta\left(q^{+}-q^{+}\right) \delta^{(2)}\left(\mathbf{q}_{\perp}-\mathbf{q}_{\perp}^{\prime}\right) . \\
{\left[a(q), a^{\dagger}\left(q^{\prime}\right)\right]=(2 \pi)^{3} \delta\left(q^{+}-q^{\prime+}\right) \delta^{(2)}\left(\mathbf{q}_{\perp}-\mathbf{q}_{\perp}^{\prime}\right) .}
\end{gathered}
$$

Using the LF commutation relations given above and the properties of the light-front spinors given in Appendix B, we obtain the expression of the light-front Hamiltonian $P^{-}$in the particle number representation

$$
P^{-}=\sum_{\lambda} \int \frac{d q^{+} d^{2} \mathbf{q}_{\perp}}{(2 \pi)^{3}}\left(\frac{\mathbf{q}_{\perp}^{2}+m^{2}}{q^{+}}\right) b_{\lambda}^{\dagger}(q) b_{\lambda}(q)+(\text { interactions }),
$$

where, for simplicity, we have omitted from (2.18) the terms corresponding to antiquarks and gluons. We recover the LF dispersion relation $q^{-}=\left(\mathbf{q}_{\perp}^{2}+m^{2}\right) / q^{+}$for a quark or antiquark in absence of interactions and the dispersion relation for the gluon quanta $q^{-}=$ $\mathbf{q}_{\perp}^{2} / q^{+}$, which follows from the on shell relation $q^{2}=m^{2}$ and $q^{2}=0$ respectively. The LF time evolution operator $P^{-}$is thus conveniently written as a term which represents the sum of the kinetic energy of all the partons plus a sum of all the interaction terms. 
The longitudinal and transverse kinematical generators are

$$
\begin{aligned}
& P^{+}=\sum_{\lambda} \int \frac{d q^{+} d^{2} \mathbf{q}_{\perp}}{(2 \pi)^{3}} q^{+} b_{\lambda}^{\dagger}(q) b_{\lambda}(q), \\
& \mathbf{P}_{\perp}=\sum_{\lambda} \int \frac{d q^{+} d^{2} \mathbf{q}_{\perp}}{(2 \pi)^{3}} \mathbf{q}_{\perp} b_{\lambda}^{\dagger}(q) b_{\lambda}(q),
\end{aligned}
$$

and contain no interactions. For simplicity we have also omitted from (2.19) and (2.20) the contribution of the kinetic terms from antiquarks and gluons.

\subsubsection{Representation of hadrons in the light-front Fock basis}

An important advantage of light-front quantization is that a particle Fock expansion can be used as the basis for representing the physical states of QCD. The light-front Fock representation is thus an interpolating basis projecting the hadronic eigenstate onto the Fock basics of free on-shell partonic constituents. The complete basis of Fock-states $|n\rangle$ is constructed by applying free-field creation operators to the vacuum state $|0\rangle$ which has no particle content, $P^{+}|0\rangle=0, \mathbf{P}_{\perp}|0\rangle=0$. A one-particle state is defined by $|q\rangle=\sqrt{2 q^{+}} b^{\dagger}(q)|0\rangle$, so that its normalization has the Lorentz invariant form

$$
\left\langle q \mid q^{\prime}\right\rangle=2 q^{+}(2 \pi)^{3} \delta\left(q^{+}-q^{\prime+}\right) \delta^{(2)}\left(\mathbf{q}_{\perp}-\mathbf{q}_{\perp}^{\prime}\right)
$$

and this fixes our normalization. Each $n$-particle Fock state $\left|p_{i}^{+}, \mathbf{p}_{\perp i}, \lambda_{i}\right\rangle$ is an eigenstate of $P^{+}, \mathbf{P}_{\perp}$ and $J^{3}$ and it is normalized according to

$$
\left\langle p_{i}^{+}, \mathbf{p}_{\perp i}, \lambda_{i} \mid p_{i}^{\prime+}, \mathbf{p}_{\perp i}^{\prime}, \lambda_{i}^{\prime}\right\rangle=2 p_{i}^{+}(2 \pi)^{3} \delta\left(p_{i}^{+}-p_{i}^{\prime+}\right) \delta^{(2)}\left(\mathbf{p}_{\perp i}-\mathbf{p}_{\perp i}^{\prime}\right) \delta_{\lambda_{i}, \lambda_{i}^{\prime}} .
$$

We now proceed to the separation of relative and overall kinematics by introducing the partonic variables $k_{i}^{\mu}=\left(k_{i}^{+}, k_{i}^{-}, \mathbf{k}_{\perp i}\right)$ according to $k_{i}^{+}=x_{i} P^{+}, \quad \mathbf{p}_{\perp i}=x_{i} \mathbf{P}_{\perp i}+\mathbf{k}_{\perp i}$, where the longitudinal momentum fraction for each constituent is $x_{i}=k_{i}^{+} / P^{+}$(See Fig. 2.2). Momentum conservation requires that $P^{+}=\sum_{i=1}^{n} k_{i}^{+}, k_{i}^{+}>0$, or equivalently $\sum_{i=1}^{n} x_{i}=1$, and $\sum_{i=1}^{n} \mathbf{k}_{\perp i}=0$. The light-front momentum coordinates $x_{i}$ and $\mathbf{k}_{\perp i}$ are actually relative coordinates; i.e., they are independent of the total momentum $P^{+}$and $\mathbf{P}_{\perp}$ of the bound state.

The hadron state is an eigenstate of the total momentum $P^{+}$and $\mathbf{P}_{\perp}$ and the total spin projection $S^{z}$. Each hadronic eigenstate $|\psi\rangle$ is expanded in a complete Fock-state basis of noninteracting $n$-particle states $|n\rangle$ with an infinite number of components. 


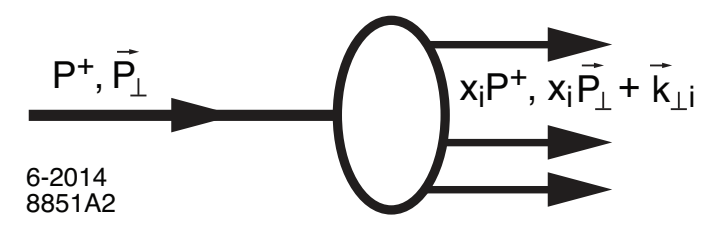

Figure 2.2: Overall and relative partonic variables in a hadronic bound state.

For example, a proton with four-momentum $P^{\mu}=\left(P^{+}, P^{-}, \mathbf{P}_{\perp}\right)$ is described by the expansion

$$
\left|\psi\left(P^{+}, \mathbf{P}_{\perp}, S_{z}\right)\right\rangle=\sum_{n, \lambda_{i}} \iint\left[d x_{i}\right]\left[d^{2} \mathbf{k}_{\perp i}\right] \frac{1}{\sqrt{x_{i}}} \psi_{n}\left(x_{i}, \mathbf{k}_{\perp i}, \lambda_{i}\right)\left|n: x_{i} P^{+}, x_{i} \mathbf{P}_{\perp}+\mathbf{k}_{\perp i}, \lambda_{i}\right\rangle,
$$

where the sum is over all Fock states and helicities, beginning with the valence state; e.g., $n \geq 3$ for baryons. The measure of the constituents phase-space momentum integration is

$$
\begin{gathered}
\int\left[d x_{i}\right] \equiv \prod_{i=1}^{n} \int d x_{i} \delta\left(1-\sum_{j=1}^{n} x_{j}\right) \\
\int\left[d^{2} \mathbf{k}_{\perp i}\right] \equiv \prod_{i=1}^{n} \int \frac{d^{2} \mathbf{k}_{\perp i}}{2(2 \pi)^{3}} 16 \pi^{3} \delta^{(2)}\left(\sum_{j=1}^{n} \mathbf{k}_{\perp j}\right) .
\end{gathered}
$$

The coefficients of the Fock expansion

$$
\psi_{n}\left(x_{i}, \mathbf{k}_{\perp i}, \lambda_{i}\right)=\left\langle n: x_{i}, \mathbf{k}_{\perp i}, \lambda_{i} \mid \psi\right\rangle
$$

are frame independent; i.e., the form of the LFWFs is independent of the total longitudinal and transverse momentum $P^{+}$and $\mathbf{P}_{\perp}$ of the hadron and depend only on the partonic coordinates: the longitudinal momentum fraction $x_{i}$, the transverse momentum $\mathbf{k}_{\perp i}$, and $\lambda_{i}$, the projection of the constituent's spin along the $z$ direction. The wave function $\psi_{n}\left(x_{i}, \mathbf{k}_{\perp i}, \lambda_{i}\right)$ represents the probability amplitudes to find on-mass-shell constituents $i$ in a specific light-front Fock state $|n\rangle$ with longitudinal momentum $x_{i} P^{+}$, transverse momentum $x_{i} \mathbf{P}_{\perp}+\mathbf{k}_{\perp i}$ and helicity $\lambda_{i}$ in a given hadron. Since the boundary conditions are specified in the null-plane, the ability to specify wave functions simultaneously in any frame is a special feature of light-front quantization.

Each constituent of the light-front wave function $\psi_{n}\left(x_{i}, \mathbf{k}_{\perp i}, \lambda_{i}\right)$ of a hadron is on its respective mass shell $k_{i}^{2}=m_{i}^{2}$, where $k_{\mu i} k_{i}^{\mu}=k_{i}^{+} k_{i}^{-}-\mathbf{k}_{\perp i}^{2}$, thus each single particle state 
has four-momentum

$$
k_{i}^{\mu}=\left(k_{i}^{+}, k_{i}^{-}, \mathbf{k}_{i}\right)=\left(k_{i}^{+}, \frac{\mathbf{k}_{\perp i}^{2}+m_{i}^{2}}{k_{i}^{+}}, \mathbf{k}_{i}\right), \quad \text { for } i=1,2 \cdots n
$$

However, the light-front wave function represents a state which is off the light-front energy shell, $P^{-}-\sum_{i}^{n} k_{i}^{-}<0$, for a stable hadron. In fact, the invariant mass of the constituents in each $n$-particle Fock state is given by

$$
M_{n}^{2}=\left(\sum_{i=1}^{n} k_{i}^{\mu}\right)^{2}=\left(\sum_{i=1}^{n} k_{i}^{+}\right)\left(\sum_{i=1}^{n} k_{i}^{-}\right)-\left(\sum_{i=1}^{n} \mathbf{k}_{\perp i}\right)^{2}=\sum_{i=1}^{n} \frac{\mathbf{k}_{\perp i}^{2}+m_{i}^{2}}{x_{i}}
$$

and is a measure of the off-energy shell of the bound state, with $M_{n}^{2}$ in general different from the hadron bound-state mass $P_{\mu} P^{\mu}=M^{2}$.

The hadron state is normalized according to

$$
\left\langle\psi\left(P^{+}, \mathbf{P}_{\perp}, S^{z}\right) \mid \psi\left(P^{\prime+}, \mathbf{P}_{\perp}^{\prime}, S^{z^{\prime}}\right)\right\rangle=2 P^{+}(2 \pi)^{3} \delta_{S^{z}, S^{z^{\prime}}} \delta\left(P^{+}-P^{\prime+}\right) \delta^{(2)}\left(\mathbf{P}_{\perp}-\mathbf{P}_{\perp}^{\prime}\right)
$$

Thus, the normalization of the LFWFs is determined by

$$
\sum_{n} \int\left[d x_{i}\right]\left[d^{2} \mathbf{k}_{\perp i}\right]\left|\psi_{n}\left(x_{i}, \mathbf{k}_{\perp i}\right)\right|^{2}=1
$$

where the internal-spin indices have been suppressed.

The constituent spin and orbital angular momentum properties of the hadrons are also encoded in the LFWFs $\psi_{n}\left(x_{i}, \mathbf{k}_{\perp i}, \lambda_{i}\right)$ which obey the total orbital angular momentum sum rule [160]

$$
J^{z}=\sum_{i=1}^{n} S_{i}^{z}+\sum_{i=1}^{n-1} L_{i}^{z}
$$

since there are only $n-1$ relative angular momenta in an $n$-particle light-front Fock state in the sum (2.31). The internal spins $S_{i}^{z}$ are denoted as $\lambda_{i}$. The orbital angular momenta have the operator form

$$
L_{i}^{z}=-i\left(\frac{\partial}{\partial k_{i}^{x}} k_{i}^{y}-\frac{\partial}{\partial k_{i}^{y}} k_{i}^{x}\right)
$$

Since the total angular momentum projection $J^{z}$ in the light front is a kinematical operator, it is conserved Fock state by Fock state and by every interaction in the LF Hamiltonian. In the light-cone gauge $A^{+}=0$, the gluons only have physical angular 
momentum projections $S^{z}= \pm 1$ and the orbital angular momentum of quark and gluons is defined unambiguously [73].

\subsection{Semiclassical approximation to QCD in the light front}

Our goal is to find a semiclassical approximation to strongly coupled QCD dynamics and derive a simple relativistic wave equation to compute hadronic bound states and other hadronic properties. To this end it is necessary to reduce the multiple particle eigenvalue problem of the LF Hamiltonian (2.7) to an effective light-front Schrödinger equation, instead of diagonalizing the full Hamiltonian. The central problem then becomes the derivation of the effective interaction, which acts only on the valence sector of the theory and has, by definition, the same eigenvalue spectrum as the initial Hamiltonian problem. For carrying out this program in the front from, one must systematically express the higher-Fock components as functionals of the lower ones. This method has the advantage that the Fock space is not truncated and the symmetries of the Lagrangian are preserved [161]. The method is similar to the methods used in many-body problems in nuclear physics to reduce the great complexity of a dynamical problem with a large number of degrees of freedom to an effective model with fewer degrees of freedom [162]. The same method is used in QED; for example, the reduction of the higher Fock states of muonium $\mu^{+} e^{-}$to an effective $\mu^{+} e^{-}$equation introduces interactions which yield the hyperfine splitting, Lamb shift, and other corrections to the Coulomb-dominated potential.

In principle one should determine the effective potential from the two-particle irreducible $q \bar{q} \rightarrow q \bar{q}$ Greens' function for a pion. In particular, the reduction from higher Fock states in the intermediate states would lead to an effective interaction $U$ for the valence $|q \bar{q}\rangle$ Fock state of the pion [161]. However, in order to capture the nonperturbative dynamics one most integrate out all higher Fock states, corresponding to an infinite number of degrees of freedom - a formidable problem. This is apparent, for example, if one identifies the sum of infrared sensitive " $\mathrm{H}$ " diagrams as the source of the effective potential, since the horizontal rungs correspond to an infinite number of higher gluonic Fock states $[163,164]$. A related approach for determining the valence light-front wave function and studying the effects of higher Fock states without truncation has been given in Ref. [165]. 
We will describe below a simple procedure which allows us to reduce the strongly correlated multi-parton bound-state problem in light-front QCD into an effective onedimensional problem [74]. To follow this procedure, it is crucial to identify as the key dynamical variable, the invariant mass $M_{n}^{2}(2.28), M_{n}^{2}=\left(k_{1}+k_{2}+\cdots k_{n}\right)^{2}$, which controls the bound state. In fact, the LFWF is of-shell in $P^{-}$and consequently in the invariant mass. Alternatively, it is useful to consider its canonical conjugate invariant variable in impact space. This choice of variable will also allow us to separate the dynamics of quark and gluon binding from the kinematics of constituent spin and internal orbital angular momentum [74].

For an $n$-Fock component $\psi\left(k_{1}, k_{2}, \cdots, k_{n}\right)$ we make the substitution

$$
\psi_{n}\left(k_{1}, k_{2}, \cdots, k_{n}\right) \rightarrow \phi_{n}\left(\left(k_{1}+k_{2}+\cdots k_{n}\right)^{2}\right), \quad m_{q} \rightarrow 0 .
$$

Using this semiclassical approximation, and in the limit of zero quark masses, the $n$ particle bound-state problem is reduced effectively to a single-variable LF quantum mechanical wave equation [74], which describes the bound-state dynamics of light hadrons in terms of an effective confining interaction $U$ defined at equal LF time. In this semiclassical approximation there is no particle creation or absorption.

Let us outline how this reduction is actually carried out in practice. We compute $M^{2}$ from the hadronic matrix element $\left\langle\psi\left(P^{\prime}\right)\left|P_{\mu} P^{\mu}\right| \psi(P)\right\rangle=M^{2}\left\langle\psi\left(P^{\prime}\right) \mid \psi(P)\right\rangle$, expanding the initial and final hadronic states in terms of their Fock components using (2.23). The computation is simplified in the frame $P=\left(P^{+}, M^{2} / P^{+}, \overrightarrow{0}_{\perp}\right)$ where $H_{L F}=P^{2}=$ $P^{+} P^{-}$. Using the normalization condition (2.22) for each individual constituent and after integration over the internal coordinates of the $n$ constituents for each Fock state in the $\mathbf{P}_{\perp}=0$ frame, one finds [74]

$$
M^{2}=\sum_{n} \int\left[d x_{i}\right]\left[d^{2} \mathbf{k}_{\perp i}\right] \sum_{a=1}^{n}\left(\frac{\mathbf{k}_{\perp a}^{2}+m_{a}^{2}}{x_{a}}\right)\left|\psi_{n}\left(x_{i}, \mathbf{k}_{\perp i}\right)\right|^{2}+\text { (interactions) }
$$

plus similar terms for antiquarks and gluons $\left(m_{g}=0\right)$. The integrals in (2.34) are over the internal coordinates of the $n$ constituents for each Fock state with the phase space normalization (2.30). Since the LF kinetic energy has a finite value in each Fock state, it follows that the LFWFs of bound states $\psi_{n}\left(x_{i}, \mathbf{k}_{\perp i}\right)$ have the small momentum fraction- $x$ boundary conditions $\psi_{n}\left(x_{i}, \mathbf{k}_{\perp i}\right) \rightarrow\left(x_{i}\right)^{\alpha}$ with $\alpha \geq \frac{1}{2}$ in the limit $x_{i} \rightarrow 0$ [166].

It is useful to express (2.34) in terms of $n-1$ independent transverse impact variables $\mathbf{b}_{\perp j}, j=1,2, \ldots, n-1$, conjugate to the relative coordinates $\mathbf{k}_{\perp i}$ using the Fourier 
expansion [167]

$$
\psi_{n}\left(x_{j}, \mathbf{k}_{\perp j}\right)=(4 \pi)^{(n-1) / 2} \prod_{j=1}^{n-1} \int d^{2} \mathbf{b}_{\perp j} \exp \left(i \sum_{k=1}^{n-1} \mathbf{b}_{\perp k} \cdot \mathbf{k}_{\perp k}\right) \psi_{n}\left(x_{j}, \mathbf{b}_{\perp j}\right)
$$

where $\sum_{i=1}^{n} \mathbf{b}_{\perp i}=0$. We find

$M^{2}=\sum_{n} \prod_{j=1}^{n-1} \int d x_{j} d^{2} \mathbf{b}_{\perp j} \psi_{n}^{*}\left(x_{j}, \mathbf{b}_{\perp j}\right) \sum_{a=1}^{n}\left(\frac{-\nabla_{\mathbf{b}_{\perp a}}^{2}+m_{a}^{2}}{x_{a}}\right) \psi_{n}\left(x_{j}, \mathbf{b}_{\perp j}\right)+$ (interactions)

where the normalization in impact space is defined by

$$
\sum_{n} \prod_{j=1}^{n-1} \int d x_{j} d^{2} \mathbf{b}_{\perp j}\left|\psi_{n}\left(x_{j}, \mathbf{b}_{\perp j}\right)\right|^{2}=1
$$

The simplest example is a two-parton hadronic bound state. If we want to reduce further the dynamics to a single-variable problem, we must take the limit of quark masses to zero. In the limit $m_{q} \rightarrow 0$ we find

$$
\begin{aligned}
M^{2} & =\int_{0}^{1} d x \int \frac{d^{2} \mathbf{k}_{\perp}}{16 \pi^{3}} \frac{\mathbf{k}_{\perp}^{2}}{x(1-x)}\left|\psi\left(x, \mathbf{k}_{\perp}\right)\right|^{2}+(\text { interactions }) \\
& =\int_{0}^{1} \frac{d x}{x(1-x)} \int d^{2} \mathbf{b}_{\perp} \psi^{*}\left(x, \mathbf{b}_{\perp}\right)\left(-\nabla_{\mathbf{b}_{\perp}}^{2}\right) \psi\left(x, \mathbf{b}_{\perp}\right)+(\text { interactions }),
\end{aligned}
$$

with normalization

$$
\int_{0}^{1} d x \int \frac{d^{2} \mathbf{k}_{\perp}}{16 \pi^{3}}\left|\psi\left(x, \mathbf{k}_{\perp}\right)\right|^{2}=\int_{0}^{1} d x \int d^{2} \mathbf{b}_{\perp}\left|\psi\left(x, \mathbf{b}_{\perp}\right)\right|^{2}=1 .
$$

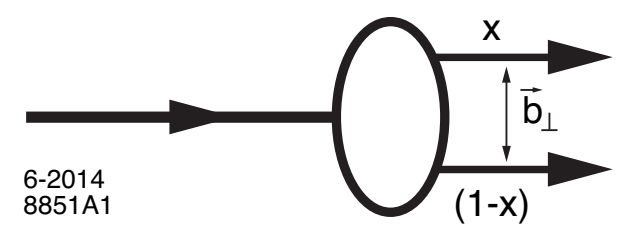

Figure 2.3: Relative $q-\bar{q}$ variables in impact space for a pion bound state.

For $n=2$, the invariant mass $(2.28)$ is $M_{q \bar{q}}^{2}=\frac{\mathbf{k}_{\perp}^{2}}{x(1-x)}$. Similarly, in impact space the relevant variable for a two-parton state is $\zeta^{2}=x(1-x) \mathbf{b}_{\perp}^{2}$, the invariant separation 
between the quark and antiquark (See Fig. 2.3). Thus, to first approximation, LF dynamics depend only on the boost invariant variable $M_{n}$ or $\zeta$, and the dynamical properties are encoded in the hadronic LF wave function $\phi(\zeta)$

$$
\psi(x, \zeta, \varphi)=e^{i L \varphi} X(x) \frac{\phi(\zeta)}{\sqrt{2 \pi \zeta}}
$$

where we have factored out the longitudinal $X(x)$ and orbital dependence from the LFWF $\psi$. This is a natural factorization in the light front since the corresponding canonical generators, the longitudinal and transverse generators $P^{+}$and $\mathbf{P}_{\perp}$ and the $z$-component of the orbital angular momentum $J^{z}$ are kinematical generators which commute with the LF Hamiltonian generator $P^{-}$. From (2.39) the normalization of the transverse and longitudinal modes is given by

$$
\begin{aligned}
\langle\phi \mid \phi\rangle & =\int d \zeta \phi^{2}(\zeta)=1 \\
\langle X \mid X\rangle & =\int_{0}^{1} d x x^{-1}(1-x)^{-1} X^{2}(x)=1 .
\end{aligned}
$$

To proceed, we write the Laplacian operator in $(2.38)$ in polar coordinates $(\zeta, \varphi)$

$$
\nabla_{\zeta}^{2}=\frac{1}{\zeta} \frac{d}{d \zeta}\left(\zeta \frac{d}{d \zeta}\right)+\frac{1}{\zeta^{2}} \frac{\partial^{2}}{\partial \varphi^{2}}
$$

and factor out the angular dependence of the modes in terms of the $S O(2)$ Casimir representation $L^{2}$ of orbital angular momentum in the transverse plane. Using (2.40) we find $[74]$

$$
M^{2}=\int d \zeta \phi^{*}(\zeta) \sqrt{\zeta}\left(-\frac{d^{2}}{d \zeta^{2}}-\frac{1}{\zeta} \frac{d}{d \zeta}+\frac{L^{2}}{\zeta^{2}}\right) \frac{\phi(\zeta)}{\sqrt{\zeta}}+\int d \zeta \phi^{*}(\zeta) U(\zeta) \phi(\zeta)
$$

where $L=\left|L^{z}\right|$. In writing the above equation we have summed up all the complexity of the interaction terms in the QCD Hamiltonian (2.10) in the introduction of the effective potential $U(\zeta)$ which acts in the valence state, and which should enforce confinement at some IR scale, which determines the QCD mass gap. The light-front eigenvalue equation $P_{\mu} P^{\mu}|\phi\rangle=M^{2}|\phi\rangle$ is thus a light-front wave equation for $\phi$

$$
\left(-\frac{d^{2}}{d \zeta^{2}}-\frac{1-4 L^{2}}{4 \zeta^{2}}+U(\zeta)\right) \phi(\zeta)=M^{2} \phi(\zeta)
$$


a relativistic single-variable LF quantum-mechanical wave equation. Its eigenmodes $\phi(\zeta)$ determine the hadronic mass spectrum and represent the probability amplitude to find the partons at transverse impact separation $\zeta$, the invariant separation between pointlike constituents within the hadron [75] at equal LF time. This equation is an effective twoparticle wave equation where an infinite number of higher Fock states [161] and retarded interactions are incorporated in the light-front effective potential, which acts on the valence states. In practice, computing the effective potential from QCD is a formidable task and other methods have to be devised to incorporate the essential dynamics from confinement. The effective interaction potential in (2.45) is instantaneous in LF time $x^{+}$, not instantaneous in ordinary time $x^{0}$. The LF potential thus satisfies causality, unlike the instantaneous Coulomb interaction appearing in atomic physics.

If $L^{2}<0$, the LF Hamiltonian defined in Eq. (2.7) is unbounded from below $\left\langle\phi\left|P_{\mu} P^{\mu}\right| \phi\right\rangle<0$, and the spectrum contains an infinite number of unphysical negative values of $M^{2}$ which can be arbitrarily large. As $M^{2}$ increases in absolute value, the particle becomes localized within a very small region near $\zeta=0$, if the effective potential vanishes at small $\zeta$. For $M^{2} \rightarrow-\infty$ the particle is localized at $\zeta=0$, the particle "falls towards the center" [168]. The critical value $L=0$ corresponds to the lowest possible stable solution, the ground state of the light-front Hamiltonian. It is important to notice that in the light front, the $S O(2)$ Casimir for orbital angular momentum $L^{2}$ is a kinematical quantity, in contrast to the usual $S O(3)$ Casimir $L(L+1)$ from nonrelativistic physics which is rotational, but not boost invariant. The $S O(2)$ Casimir form $L^{2}$ corresponds to the group of rotations in the transverse LF plane. Indeed, the Casimir operator for $S O(N)$ is $L(L+N-2)$.

If we compare the invariant mass in the instant form in the hadron center-of-mass system, $\mathbf{P}=0, M_{q \bar{q}}^{2}=4 m_{q}^{2}+4 \mathbf{p}^{2}$, with the invariant mass in the front form in the constituent rest frame, $\mathbf{p}_{q}+\mathbf{p}_{\bar{q}}=0$ for equal quark-antiquark masses ${ }^{3}$, we obtain the relation [97]

$$
U=V^{2}+2 \sqrt{\mathbf{p}^{2}+m_{q}^{2}} V+2 V \sqrt{\mathbf{p}^{2}+m_{q}^{2}}
$$

where we identify $\mathbf{p}_{\perp}^{2}=\frac{\mathbf{k}_{\perp}^{2}}{4 x(1-x)}, p_{3}=\frac{m_{q}(x-1 / 2)}{\sqrt{x(1-x)}}$, and $V$ is the effective potential in the instant form. Thus, for small quark masses a linear instant-form potential $V$ implies a harmonic front-form potential $U$ at large distances. One can also show how the two-dimensional front-form harmonic oscillator potential for massless quarks takes on a

\footnotetext{
${ }^{3}$ Notice that the hadron center-of-mass frame and the constituent rest frame are not identical in the front form of dynamics since the third component of momentum is not conserved in the light front (See Ref. [97] and references therein.
} 
three-dimensional form when the quarks have mass since the third space component is conjugate to $p_{3}$, which has an infinite range for $m \neq 0$ [97].

Extension of the results to arbitrary $n$ follows from the $x$-weighted definition of the transverse impact variable of the $n-1$ spectator system [75] given by the LF cluster decomposition

$$
\zeta=\sqrt{\frac{x}{1-x}}\left|\sum_{j=1}^{n-1} x_{j} \mathbf{b}_{\perp j}\right|,
$$

where $x=x_{n}$ is the longitudinal momentum fraction of the active quark. One can also generalize the equations to allow for the kinetic energy of massive quarks using Eqs. (2.34) or (2.36) as discussed in Sec. 2.4.1 below. In this case, however, the longitudinal mode $X(x)$ does not decouple from the effective LF bound-state equations.

\subsubsection{Inclusion of light quark masses}

The noticeable simplicity of the transverse single-variable light-front wave equation derived from the bound-state Hamiltonian equation of motion in Sec. 2.4 is lost when we consider massive quarks, as longitudinal LF variables have to be taken into account as well. In the limit of massless quarks the scheme is very concise and unique: in the semiclassical approximation the underlying conformal symmetry of QCD determines the dynamics (Chapter 3) and there is an exact agreement of the AdS equations of motion and the light-front Hamiltonian (Chapter 4). However, as we will discuss here, the inclusion of small quark masses can still be treated in a simple way following the semiclassical approximation described above.

As it is clear from (2.46), the effective light-front confining interaction $U$ has a strong dependence on heavy quark masses and consequently in the longitudinal variables ${ }^{4}$. However, for small quark masses - as compared to the hadronic scale, one expects that the effective confinement interaction in the quark masses is unchanged to first order. In this approximation the confinement potential only depends on the transverse invariant variable $\zeta$, and the transverse dynamics are unchanged. The partonic shift in

\footnotetext{
${ }^{4}$ This connection was used in Ref. [169] to construct a light-front potential for heavy quarkonia.
} 
the hadronic mass is computed straightforwardly from (2.34) or (2.36)

$$
\begin{aligned}
\Delta M^{2} & =\sum_{n} \int\left[d x_{i}\right]\left[d^{2} \mathbf{k}_{\perp i}\right] \sum_{a=1}^{n} \frac{m_{a}^{2}}{x_{a}}\left|\psi_{n}\left(x_{i}, \mathbf{k}_{\perp i}\right)\right|^{2} \\
& =\sum_{n} \prod_{j=1}^{n-1} \int d x_{j} d^{2} \mathbf{b}_{\perp j} \sum_{a=1}^{n} \frac{m_{a}^{2}}{x_{a}}\left|\psi_{n}\left(x_{j}, \mathbf{b}_{\perp j}\right)\right|^{2} \\
& \equiv\left\langle\psi\left|\sum_{a} \frac{m_{a}^{2}}{x_{a}}\right| \psi\right\rangle,
\end{aligned}
$$

where $\Delta M^{2}=M^{2}-M_{0}^{2}$. Here $M_{0}^{2}$ is the value of the hadronic mass computed in the limit of zero quark masses. This expression is identical to the Weisberger result for a partonic mass shift [170]. Notice that this result is exact to first order in the light-quark mass if the sum in (2.48) is over all Fock states $n$.

For simplicity, we consider again the case of a meson bound-state of a quark and an antiquark with longitudinal momentum $x$ and $1-x$ respectively. To lowest order in the quark masses we find

$$
\Delta M^{2}=\int_{0}^{1} \frac{d x}{x(1-x)}\left(\frac{m_{q}^{2}}{x}+\frac{m_{\bar{q}}^{2}}{1-x}\right) X^{2}(x)
$$

using the normalization (2.42). The quark masses $m_{q}$ and $m_{\bar{q}}$ in (2.49) are effective quark masses from the renormalization due to the reduction of higher Fock states as functionals of the valence state [161], not "current" quark masses, i.e., the quark masses appearing in the QCD Lagrangian.

As it will be shown in Chapter 6, the factor $X(x)$ in the LFWF in (2.40) can be determined in the limit of massless quarks from the precise mapping of light-front amplitudes for arbitrary momentum transfer $Q$. Its form is $X(x)=x^{\frac{1}{2}}(1-x)^{\frac{1}{2}}$ [75]. This expression of the LFWF gives a divergent expression for the partonic mass shift (2.49), and, evidently, realistic effective two-particle wave functions have to be additionally suppressed at the end-points $x=0$ and $x=1$. As pointed out in [138], a key for this modification is suggested by the construction of the light-front wave functions discussed above. It relies on the assumption that the essential dynamical variable which controls the bound state wave function in momentum space is the invariant mass (2.28), which determines the off-energy shell behavior of the bound state. For the effective two-body 
bound state the inclusion of light quark masses amounts to the replacement

$$
M_{q \bar{q}}^{2}=\frac{\mathbf{k}_{\perp}^{2}}{x(1-x)} \rightarrow \frac{\mathbf{k}_{\perp}^{2}}{x(1-x)}+\frac{m_{q}^{2}}{x}+\frac{m_{\bar{q}}^{2}}{1-x}
$$

in the LFWF in momentum space, $\psi_{q \bar{q}}\left(x, \mathbf{k}_{\perp}\right)$, which is then Fourier transformed to impact space. We will come back to this point in Chapter 5 for the specific models dictated by conformal invariance. The longitudinal dynamics in presence of quark masses has also been discussed in Ref. [171].

In the next chapter we will show how the form of the effective confinement potential is uniquely determined from the algebraic structure of an effective one-dimensional quantum field theory, which encodes the underlying conformality of the classical QCD Lagrangian. In subsequent chapters we will discuss the connection of effective gravity theories in AdS space with the light-front results presented in this section. 


\section{Chapter 3}

\section{Conformal Quantum Mechanics and Light-Front Dynamics}

As we have emphasized in the introduction, conformal symmetry plays a special, but somehow hidden, role in QCD. The classical Lagrangian is, in the limit of massless quarks, invariant under conformal transformations $[5,6]$. The symmetry, however, is broken by quantum corrections. Indeed, the need for renormalization of the theory introduces a scale $\Lambda_{\mathrm{QCD}}$ which leads to the "running coupling" $\alpha_{s}\left(Q^{2}\right)$ and asymptotic freedom $[40,41]$ for large values of $Q^{2}, Q^{2} \gg \Lambda_{\mathrm{QCD}}^{2}$, a mechanism conventionally named "dimensional transmutation". But there are theoretical and phenomenological indications that at large distances, or small values of $Q^{2}, Q^{2} \leq \Lambda_{\mathrm{QCD}}^{2}$, where the string tension has formed, the QCD $\beta$-function vanishes and scale invariance is in some sense restored (See for example Ref. [76] and references therein). Since we are interested in a semiclassical approximation to nonperturbative QCD, analogous to the quantum mechanical wave equations in atomic physics, it is natural to have a closer look at conformal quantum mechanics, a conformal field theory in one dimension. De Alfaro, Fubini and Furlan [94] have obtained remarkable results which, extended to light-front holographic QCD [74, 95], give important insights into the QCD confining mechanism. It turns out that it is possible to introduce a scale in the light-front Hamiltonian, by modifying the variable of dynamical evolution and nonetheless the underlying action remains conformally invariant. Remarkably this procedure determines uniquely the form of the light-front effective potential and correspondingly the modification of AdS space. 


\subsection{One-dimensional conformal field theory}

Our aim is to incorporate in a one-dimensional quantum field theory - as an effective theory, the fundamental conformal symmetry of the four-dimensional classical QCD Lagrangian in the limit of massless quarks. We will require that the corresponding one-dimensional effective action, which encodes the chiral symmetry of QCD, remains conformally invariant. De Alfaro et al. [94] investigated in detail the simplest scaleinvariant model, one-dimensional field theory, namely

$$
A[Q]=\frac{1}{2} \int d t\left(\dot{Q}^{2}-\frac{g}{Q^{2}}\right)
$$

where $\dot{Q} \equiv d Q / d t$. Since the action is dimensionless, the dimension of the field $Q$ must be half the dimension of the "time" variable $t, \operatorname{dim}[Q]=\frac{1}{2} \operatorname{dim}[t]$, and the constant $g$ is dimensionless. The translation operator in $t$, the Hamiltonian, is

$$
H=\frac{1}{2}\left(\dot{Q}^{2}+\frac{g}{Q^{2}}\right)
$$

where the field momentum operator is $P=\dot{Q}$, and therefore the equal time commutation relation is

$$
[Q(t), \dot{Q}(t)]=i
$$

The equation of motion for the field operator $Q(t)$ is given by the usual quantum mechanical evolution

$$
i[H, Q(t)]=\frac{d Q(t)}{d t}
$$

In the Schrödinger picture with $t$-independent operators and $t$-dependent state vectors the evolution is given by

$$
H|\psi(t)\rangle=i \frac{d}{d t}|\psi(t)\rangle
$$

Using the representation of the field operators $Q$ and $P=\dot{Q}$ given by the substitution $Q(0) \rightarrow x, \dot{Q}(0) \rightarrow-i d / d x$ we obtain the usual quantum mechanical evolution

$$
i \frac{\partial}{\partial t} \psi(x, \tau)=H\left(x,-i \frac{d}{d x}\right) \psi(x, t)
$$


with the Hamiltonian

$$
H=\frac{1}{2}\left(-\frac{d^{2}}{d x^{2}}+\frac{g}{x^{2}}\right) .
$$

It has the same structure as the LF Hamiltonian (2.45) with a vanishing light-front potential, as expected for a conformal theory. The dimensionless constant $g$ in the action (3.1) is now related to the Casimir operator of rotations in the light-front wave equation (2.45).

However, as emphasized by dAFF, the absence of dimensional constants in (3.1) implies that the action action $A[Q]$ is invariant under a larger group of transformations, the full conformal group in one dimension, that is, under translations, dilatations, and special conformal transformations. For one dimension these can be expressed by the transformations of the variable $t$

$$
t^{\prime}=\frac{\alpha t+\beta}{\gamma t+\delta} ; \quad \alpha \delta-\beta \gamma=1
$$

and the corresponding field transformation

$$
Q^{\prime}\left(t^{\prime}\right)=\frac{Q(t)}{\gamma t+\delta}
$$

As we show in Appendix $\mathrm{C}$, the action $A[Q]$ (3.1) is indeed, up to a surface term, invariant under conformal transformations.

The constants of motion of the action are obtained by applying Noether's theorem. The three conserved generators corresponding to the invariance of the action (3.1) under the full conformal group in one dimension are (Appendix C):

1. Translations in the variable $t$ :

$$
H=\frac{1}{2}\left(\dot{Q}^{2}+\frac{g}{Q^{2}}\right)
$$

2. Dilatations:

$$
D=\frac{1}{2}\left(\dot{Q}^{2}+\frac{g}{Q^{2}}\right) t-\frac{1}{4}(\dot{Q} Q+Q \dot{Q})
$$

3. Special conformal transformations:

$$
K=\frac{1}{2}\left(\dot{Q}^{2}+\frac{g}{Q^{2}}\right) t^{2}-\frac{1}{2}(\dot{Q} Q+Q \dot{Q}) t+\frac{1}{2} Q^{2}
$$


where we have taken the symmetrized product of the classical expression $\dot{Q} Q$ because the operators have to be Hermitean. Using the commutation relations (3.3) one can check that the operators $H, D$ and $K$ do indeed fulfill the algebra of the generators of the one-dimensional conformal group $\operatorname{Conf}\left(R^{1}\right)$ as it shown in Appendix C:

$$
[H, D]=i H, \quad[H, K]=2 i D, \quad[K, D]=-i K .
$$

The conformal group in one dimension is locally isomorphic to the group $S O(2,1)$, the Lorentz group in $2+1$ dimensions. In fact, by introducing the combinations

$$
L^{0,-1}=\frac{1}{2}\left(a H+\frac{1}{a} K\right), \quad L^{1,0}=\frac{1}{2}\left(-a H+\frac{1}{a} K\right), \quad L^{1,-1}=D,
$$

one sees that that the generators $L^{0,-1}, L^{1,0}$ and $L^{1,-1}$ satisfy the commutation relations of the algebra of the generators of the group $S O(2,1)$

$$
\left[L^{0,-1}, L^{1,0}\right]=i L^{1,-1}, \quad\left[L^{0,-1}, L^{1,-1}\right]=-i L^{1,0}, \quad\left[L^{1,0}, L^{1,-1}\right]=-i L^{0,-1},
$$

where $L^{1, i}, i=-1,0$ are the boosts in the space direction 1 and $L^{0,-1}$ the rotation in the $(-1,0)$ plane (See Sec. A.2.3). The rotation operator $L^{0,-1}$ is compact and has thus a discrete spectrum with normalizable eigenfunctions. Since the dimensions of $H$ and $K$ are different, the constant $a$ has the dimension of $t$. In fact, the relation between the generators of the conformal group and the generators of $S O(2,1)$ suggests that the scale a may play a fundamental role [94]. This superposition of different invariants of motion, which implies the introduction of a scale, opens the possibility to construct a confining semiclassical theory based on an underlying conformal symmetry.

Generally one can construct a new "Hamiltonian" by any superposition of the three constants of motion

$$
G=u H+v D+w K .
$$

The new Hamiltonian acts on the state vector, but its evolution involves a new "time" variable. To determine the action of the generator $G$ (3.16) on the state vector, we consider the infinitesimal transformation properties of the generators $H, D$ and $K$ given by (C.14) in Appendix C

$$
e^{-i \epsilon G}|\psi(t)\rangle=|\psi(t)\rangle+\epsilon\left(u+v t+w t^{2}\right) \frac{d}{d t}|\psi(t)\rangle+O\left(\epsilon^{2}\right) .
$$


Thus, we recover the usual quantum mechanical evolution for the state vector

$$
G|\psi(\tau)\rangle=i \frac{d}{d \tau}|\psi(\tau)\rangle
$$

provided that we introduce a new time variable $\tau$ defined through [94]

$$
d \tau=\frac{d t}{u+v t+w t^{2}} .
$$

Likewise, we can find the evolution of the field $Q(t)$ from the combined action of the generators $H, D$ and $K$. Using the equations (C.15) in Appendix $\mathrm{C}$ we find

$$
i[G, Q(t)]=\frac{d Q(t)}{d t}\left(u+v t+w t^{2}\right)-Q(t) \frac{1}{2} \frac{d}{d t}\left(u+v t+w t^{2}\right) .
$$

and thus the Heisenberg equation of motion

$$
i[G, q(\tau)]=\frac{d q(\tau)}{d \tau},
$$

where the rescaled field $q(\tau)$ is given by

$$
q(\tau)=\frac{Q(t)}{\left(u+v t+w t^{2}\right)^{1 / 2}} .
$$

From (3.3) it follows that the new field also satisfies the usual quantization condition

$$
[q(\tau), \dot{q}(\tau)]=i,
$$

where $\dot{q}=d q / d \tau$.

If one expresses the action $A$ (3.1) in terms of the transformed fields $q(\tau)$, one finds

$$
\begin{aligned}
A[Q] & =\frac{1}{2} \int d \tau\left(\dot{q}^{2}-\frac{g}{q^{2}}-\frac{4 u \omega-v^{2}}{4} q^{2}\right)+A_{\text {surface }} \\
& =A[q]+A_{\text {surface }}
\end{aligned}
$$

where $A_{\text {surface }}$ is given through (C.17). Thus, up to a surface term, which does not modify the equations of motion, the action (3.1) remains unchanged under the transformations 
(3.19) and (3.22). However, the Hamiltonian derived from $A[q]$,

$$
G=\frac{1}{2}\left(\dot{q}^{2}+\frac{g}{q^{2}}+\frac{4 u w-v^{2}}{4} q^{2}\right)
$$

contains the factor $4 u w-v^{2}$ which breaks the scale invariance. It is a compact operator for $4 u w-v^{2}>0$. It is important to notice that the appearance of the generator of special conformal transformations $K$ in (3.16) is essential for confinement. This stresses the importance of the total derivative modifying the Lagrangian under the special conformal transformation (See (C.3)).

We use the Schrödinger picture from the representation of $q$ and $p=\dot{q}: q \rightarrow y, \dot{q} \rightarrow$ $-i d / d y$

$$
i \frac{\partial}{\partial \tau} \psi(y, \tau)=G\left(y,-i \frac{d}{d y}\right) \psi(y, \tau),
$$

with the corresponding Hamiltonian

$$
G=\frac{1}{2}\left(-\frac{d^{2}}{d y^{2}}+\frac{g}{y^{2}}+\frac{4 u \omega-v^{2}}{4} y^{2}\right)
$$

in the Schrödinger representation [94]. For $g \geq-1 / 4$ and $4 u w-v^{2}>0$ the operator (3.27) has a discrete spectrum. It is remarkable that it is indeed possible to construct a compact operator starting from the conformal action (3.1), without destroying the scale invariance of the action itself.

We go now back to the original field operator $Q(t)$ in (3.1). From (3.22) one obtains the relations

$$
q(0)=\frac{Q(0)}{\sqrt{u}}, \quad \dot{q}(0)=\sqrt{u} \dot{Q}(0)-\frac{v}{2 \sqrt{u}} Q(0),
$$

and thus from (3.25) we obtain

$$
\begin{aligned}
G(Q, \dot{Q}) & =\frac{1}{2} u\left(\dot{Q}^{2}+\frac{g}{Q^{2}}\right)-\frac{1}{4} v(Q \dot{Q}+\dot{Q} Q)+\frac{1}{2} w Q^{2} \\
& =u H+v D+w K
\end{aligned}
$$

at $t=0$. We thus recover the evolution operator (3.16) which describes, like (3.25), the evolution in the variable $\tau$ (3.18), but expressed in terms of the original field $Q$. The change in the time variable (3.19), required by the conformal invariance of the action, implies the change of the original Hamiltonian (3.2) to (3.29).

With the realization of the operator $Q(0)$ in the state space with wave functions 
$\psi(x, \tau)$ and the substitution $Q(0) \rightarrow x$ and $\dot{Q}(0) \rightarrow-i \frac{d}{d x}$ we obtain

$$
i \frac{\partial}{\partial \tau} \psi(x, \tau)=G\left(x,-i \frac{d}{d x}\right) \psi(x, \tau)
$$

and from (3.29) the Hamiltonian

$$
G=\frac{1}{2} u\left(-\frac{d^{2}}{d x^{2}}+\frac{g}{x^{2}}\right)+\frac{i}{4} v\left(x \frac{d}{d x}+\frac{d}{d x} x\right)+\frac{1}{2} w x^{2} .
$$

The field $q(\tau)$ was only introduced as an intermediate step in order to recover the evolution equation (3.21) and the Hamiltonian (3.25) from the action (3.1) with variational methods. This shows that the essential point for confinement and the emergence of a mass gap is indeed the change from $t$ to $\tau$ as evolution parameter.

\subsection{Connection to light-front dynamics}

We can now apply the group theoretical results from the conformal algebra to the front-form ultra-relativistic bound-state wave equation obtained in Chapter 2. Comparing the Hamiltonian (3.31) with the light-front wave equation (2.45) and identifying the variable $x$ with the light-front invariant variable $\zeta$, we have to choose $u=2, v=0$ and relate the dimensionless constant $g$ to the LF orbital angular momentum, $g=L^{2}-1 / 4$, in order to reproduce the light-front kinematics. Furthermore $w=2 \lambda^{2}$ fixes the confining light-front potential to a quadratic $\lambda^{2} \zeta^{2}$ dependence.

For the Hamiltonian $G$ (3.31) mapped to the light-front Hamiltonian in (2.45), i.e., $u=2, v=0$ and $w=2 \lambda^{2}$ one has

$$
\begin{aligned}
G & =-\frac{d^{2}}{d x^{2}}+\frac{g}{x^{2}}+\lambda^{2} x^{2} \\
& =2\left(H+\lambda^{2} K\right)
\end{aligned}
$$

and the relation with the algebra of the group $S O(2,1)$ becomes particularly compelling. From the relations (3.14) follows the connection of the free Hamiltonian $H$, (3.2) with the group generators of $S O(2,1)$

$$
L^{0,-1}-L^{1,0}=a H
$$

The Hamiltonian (3.32) can be expressed as a generalization of (3.33) by replacing 
$L^{0,-1}-L^{1,0}$ by $L^{0,-1}-\chi L^{1,0}$. This generalization yields indeed

$$
L^{0,-1}-\chi L^{1,0}=\frac{1}{4} a(1+\chi) G
$$

with

$$
\lambda=\frac{1}{a^{2}} \frac{1-\chi}{1+\chi}
$$

in (3.32). Thus the confining LF Hamiltonian

$$
H_{L F}=-\frac{d^{2}}{d \zeta^{2}}+\frac{g}{\zeta^{2}}+\lambda \zeta^{2}
$$

For $\chi=1$ we recover the free case (3.33), whereas for $-1<\chi<1$ we obtain a confining LF potential. For $\chi$ outside this region, the Hamiltonian is not bounded from below.

This consideration based on the isomorphism of the conformal group in one dimension with the group $S O(2,1)$ makes the appearance of a dimensionful constant in the Hamiltonian (3.36), derived from a conformally invariant action, less astonishing. In fact, as mentioned below Eqs. (3.14) and (3.15), one has to introduce the dimensionful constant $a \neq 0$ in order to relate the generators of the conformal group with those of the group $S O(2,1)$. This constant $a$ sets the scale for the confinement strength $\lambda^{2}$ but does not determine its magnitude, as can be seen from (3.35). This value depends on $a$ as well as on the relative weight of the two generators $L^{0,-1}$ and $L^{1,0}$ in the construction of the Hamiltonian (3.34).

Since the invariant light-front Hamiltonian is the momentum square operator, $H_{L F}=$ $P^{2}=P^{+} P^{-}-\mathbf{P}_{\perp}^{2}$ with the LF evolution operator $P^{-}=\frac{\partial}{\partial x^{+}}$, it follows from the identification of $G$ (3.31) with the LF wave equation (2.45) and the Hamiltonian evolution equation (2.6), that in the LF frame $\mathbf{P}_{\perp}=\mathbf{0}$ the evolution parameter $\tau$ is proportional to the LF time $x^{+}=x^{0}+x^{3}$, namely $\tau=x^{+} / P^{+}$, where $P^{+}=P^{0}+P^{3}$ is the hadron longitudinal momentum. Therefore, the dimension of $\tau$ is that of an inverse mass squared, characteristic of the fully relativistic treatment.

In the original paper of de Alfaro et al. [94] and subsequent investigations [172, 173], the aim was not so much to obtain a confining model, but rather to investigate conformal field theories. The use of the compact operator $L^{0,-1}$, constructed inside the algebra of generators of the conformal group, served mainly to obtain a normalizable vacuum state in order to make contact with quantum fields operating in a Fock space. In this sense, the parameters $u, v$ and $w$ played only an auxiliary role. In the context of the present review, one is however primarily interested in the dynamical evolution in terms of the 
new variable $\tau$, which turns out to be proportional to the light front time $x^{+}=x^{0}+x^{3}$, therefore the dimensioned parameter $w$ plays a physical role ${ }^{1}$; namely, that of setting the hadronic scale in the LF potential.

In their discussion of the evolution operator $G$ de Alfaro et. al. mention a critical point, namely that "the time evolution is quite different from a stationary one". By this statement they refer to the fact that the variable $\tau$ is related to the variable $t$ by

$$
\tau=\frac{1}{\sqrt{2 w}} \arctan \left(t \sqrt{\frac{w}{2}}\right)
$$

a quantity which is of finite range. Thus $\tau$ has the natural interpretation in a meson as the difference of light-front times between events involving the quark and antiquark, and in principle could be measured in double parton-scattering processes [95]. Thus the finite range of $\tau$ corresponds to the finite size of hadrons due to confinement.

To sum up, the dAFF mechanism for introducing a scale makes use of the algebraic structure of one dimensional conformal field theory. A new Hamiltonian with a mass scale $\sqrt{\lambda}$ is constructed from the generators of the conformal group and its form is therefore fixed uniquely: it is, like the original Hamiltonian with unbroken dilatation symmetry, a constant of motion [94]. The essential point of this procedure is the introduction of a new evolution parameter $\tau$. The theory defined in terms of the new evolution Hamiltonian $G$ has a well-defined vacuum, but the dAFF procedure breaks Poincaré and scale invariance [172]. The symmetry breaking in this procedure is reminiscent of spontaneous symmetry breaking, however, this is not the case since, in contrast with current algebra, there are no degenerate vacua [172] (the vacuum state is chosen ab initio) and thus a massless scalar $0^{++}$state is not required. The dAFF mechanism is also different from usual explicit breaking by just adding a mass term to the Lagrangian [174].

\subsection{Conformal quantum mechanics, $S O(2,1)$ and $\mathbf{A d S}_{2}$}

The local isomorphism between the conformal group in one-dimension and the group $S O(2,1)$ is fundamental for introducing the scale for confinement in the light-front Hamiltonian. In fact, the conformal group in one dimension $\operatorname{Conf}\left(R^{1}\right)$ is locally isomor-

\footnotetext{
${ }^{1}$ This possibility was also shortly discussed in general terms by dAFF [94].
} 
phic not only to the group $S O(2,1)$, but also to the isometries of $\mathrm{AdS}_{2}{ }^{2}$. Using Table A.1, one can see explicitly the equivalence of the generators of $\mathrm{AdS}_{2}$ isometries at the $\mathrm{AdS}_{2}$ boundary, $z=0$, with the representation of the conformal generators $H, D$ and $K$ in conformal quantum mechanics given by (C.14) in Appendix C. In the limit $z \rightarrow 0$ we have:

$$
\begin{aligned}
H & =\frac{i R}{a} \frac{\partial}{\partial t} \\
D & =i\left(t \frac{\partial}{\partial t}+z \frac{\partial}{\partial z}\right) \rightarrow i t \frac{\partial}{\partial t} \\
K & =\frac{i a}{R}\left(\left(t^{2}+z^{2}\right) \frac{\partial}{\partial t}+2 z t \frac{\partial}{\partial z}\right) \rightarrow \frac{i a}{R} t^{2} \frac{\partial}{\partial t},
\end{aligned}
$$

In the equation above $a$ is the dimensionfull constant required to establish the isomorphism between the generators of the conformal group $\operatorname{Con} f\left(R^{1}\right)$ and the generators of $S O(2,1)$ given by $(3.14)$, and $R$ is the $\mathrm{AdS}_{2}$ radius; a priori two independent scales. Comparing with Eq. (C.14)) we find that the generators are indeed identical, if the scales are also identical. Thus $H=i \partial_{t}, D=i t \partial_{t}$ and $H=i t^{2} \partial_{t}$ provided that $a=R$.

In the next chapter, we shall derive bound-state equations for hadronic states from classical gravity in AdS space, where the confinement arises from distortion of this higher dimensional space. We will see there that the method discussed in this chapter fixes uniquely the modification of AdS space and not only the form of the light-front confinement potential.

\footnotetext{
${ }^{2}$ The isomorphism of the algebra of generators of the group $S O(2,1)$ and the isometries of $\mathrm{AdS}_{2}$ space is the basis of the $\mathrm{AdS}_{2} / \mathrm{CFT}_{1}$ correspondence [173].
} 


\section{Chapter 4}

\section{Higher-Spin Wave Equations and AdS Kinematics and Dynamics}

In this chapter we derive hadronic bound-state wave equations with arbitrary spin in a higher-dimensional space asymptotic to anti-de Sitter space. We give first an introductory derivation of the wave equations for scalar and vector fields. We then extend our treatment to higher spin using AdS tensors or generalized Rarita-Schwinger spinor fields in AdS for all integer and half-integer spins respectively. Our procedure takes advantage of the Lorentz frame (the local inertial frame). Further simplification is brought by the fact that physical hadrons form tensor representations in $3+1$ dimensions. In the present approach, the subsidiary conditions required to eliminate the lower-spin states from the symmetric tensors follow from the higher-dimensional Euler-Lagrange equations of motion and are not imposed. It turns out that the AdS geometry fixes the kinematical features of the theory, whereas the breaking of maximal symmetry from additional deformations of AdS space determines the dynamical features, including confinement. It will be shown that a strict separation of the two is essential in light-front holographic QCD ${ }^{1}$.

We briefly review in Appendix A the relevant elements of Riemannian geometry useful in the discussion of anti-de Sitter space and applications of the gauge/gravity correspondence, and in Appendix D we discuss technical details useful for the derivation of integer and half-integer wave equations in holographic QCD.

\footnotetext{
${ }^{1} \mathrm{~A}$ more detailed discussion of the procedures discussed here is given in Ref. [91].
} 


\subsection{Scalar and vector fields}

The derivation of the equation of motion for a scalar field in AdS is a particularly simple example. As mentioned in the introduction, in order to describe hadronic states using holographic methods, one has to break the maximal symmetry of the AdS metric, which is done by introducing a scale via a dilaton profile depending explicitly on the holographic variable $z$. For the sake of generality we mostly work in a $(d+1)$-dimensional curved space, and for all direct physical applications we take $d=4$. In Sec. 4.2 .1 we shall also consider the breaking of maximal symmetry by warping the AdS metric.

The coordinates of $\mathrm{AdS}_{d+1}$ space are the $d$-dimensional Minkowski coordinates $x^{\mu}$ and the holographic variable $z$. The combined coordinates are labeled $x^{M}=\left(x^{\mu}, z=x^{d}\right)$ with $M, N=0, \ldots, d$ the indices of the higher dimensional $d+1$ curved space, and $\mu, \nu=0,1, \ldots, d-1$ the Minkowski flat space-time indices. In Poincaré coordinates (Sec. A.2.2), the conformal AdS metric is

$$
\begin{aligned}
d s^{2} & =g_{M N} d x^{M} d x^{N} \\
& =\frac{R^{2}}{z^{2}}\left(\eta_{\mu \nu} d x^{\mu} d x^{\nu}-d z^{2}\right) .
\end{aligned}
$$

Here $g^{M N}$ is the full space metric tensor (A.57) and $\eta_{\mu \nu}=\operatorname{diag}[1,-1 \cdots-1]$ the metric tensor of Minkowski space. For a scalar field in AdS space $\Phi\left(x^{0}, \cdots x^{d-1}, z\right)$ the invariant action (up to bilinear terms) is

$$
S=\frac{1}{2} \int d^{d} x d z \sqrt{g} e^{\varphi(z)}\left(g^{M N} \partial_{M} \Phi \partial_{N} \Phi-\mu^{2} \Phi^{2}\right)
$$

where $g=\left(\frac{R}{z}\right)^{2 d+2}$ is the modulus of the determinant of the metric tensor $g_{M N}$. At this point, the $\mathrm{AdS}_{d+1}$ mass $\mu$ in (4.2) is not a physical observable and it is a priori an arbitrary parameter. The integration measure $d^{d} x d z \sqrt{g}$ is AdS invariant, and the action (4.2) is written in terms of simple derivatives, since the derivative of a scalar field transforms as a covariant vector field. For $\varphi \equiv 0$ the action is also AdS invariant, but the dilaton background $\varphi(z)$ effectively breaks the maximal symmetry of AdS (See A.2). It is a function of the holographic variable $z$ which vanishes in the conformal limit $z \rightarrow 0$. As we will show below, it is crucial that the dilaton profile is only a function of the variable $z$. This allows the separation of the overall movement of the hadron from its internal dynamics. In $\mathrm{AdS}_{5}$, this unique $z$-dependence of the dilaton allows the description of the bound-state dynamics in terms of a one-dimensional wave equation. It also enable 
us to to establish a map to the semiclassical one-dimensional approximation to lightfront QCD given by the frame-independent light-front Schrödinger equation obtained in Chapter 2.

The equations of motion for the field $\Phi(x, z)$ are obtained from the variational principle

$$
\frac{\delta S}{\delta \Phi}=\frac{1}{\sqrt{g} e^{\varphi}} \partial_{M}\left(\sqrt{g} e^{\varphi} g^{M N} \frac{\partial \mathcal{L}}{\partial\left(\partial_{N} \Phi\right)}\right)-\frac{\partial \mathcal{L}}{\partial \Phi}=0,
$$

from which one obtains the wave equation for the scalar field

$$
\left[\partial_{\mu} \partial^{\mu}-\frac{z^{d-1}}{e^{\varphi(z)}} \partial_{z}\left(\frac{e^{\varphi(z)}}{z^{d-1}} \partial_{z}\right)+\frac{(\mu R)^{2}}{z^{2}}\right] \Phi=0,
$$

where $\partial_{\mu} \partial^{\mu} \equiv \eta^{\mu \nu} \partial_{\mu} \partial_{\nu}$.

A free hadronic state in holographic QCD is described by a plane wave in physical space-time and a $z$-dependent profile function:

$$
\Phi(x, z)=e^{i P \cdot x} \Phi_{J=0}(z),
$$

with invariant hadron mass $P_{\mu} P^{\mu} \equiv \eta^{\mu \nu} P_{\mu} P_{\nu}=M^{2}$. Inserting (4.5) into the wave equation (4.4) we obtain the bound-state eigenvalue equation

$$
\left[-\frac{z^{d-1}}{e^{\varphi(z)}} \partial_{z}\left(\frac{e^{\varphi(z)}}{z^{d-1}} \partial_{z}\right)+\frac{(\mu R)^{2}}{z^{2}}\right] \Phi_{J=0}=M^{2} \Phi_{J=0}
$$

for spin $J=0$ hadronic states.

As a further example, we also derive the equations of motion for a vector field $\Phi_{M}(x, z)$. We start with the generalized Proca action in $\mathrm{AdS}_{d+1}$ space

$$
S=\int d^{d} x d z \sqrt{g} e^{\varphi(z)}\left(\frac{1}{4} g^{M R} g^{N S} F_{M N} F_{R S}-\frac{1}{2} \mu^{2} g^{M N} \Phi_{M} \Phi_{N}\right)
$$

where $F_{M N}=\partial_{M} \Phi_{N}-\partial_{N} \Phi_{M}$. The antisymmetric tensor $F_{M N}$ is covariant, since the parallel transporters in the covariant derivatives (A.14) cancel. Variation of the action leads to the equation of motion

$$
\frac{1}{\sqrt{g} e^{\varphi}} \partial_{M}\left(\sqrt{g} e^{\varphi} g^{M R} g^{N S} F_{R S}\right)+\mu^{2} g^{N R} \Phi_{R}=0
$$


together with the supplementary condition

$$
\partial_{M}\left(\sqrt{g} e^{\varphi} g^{M N} \Phi_{N}\right)=0
$$

One obtains from (4.8) and the the condition (4.9), the system of coupled differential equations $^{2}$

$$
\begin{aligned}
{\left[\partial_{\mu} \partial^{\mu}-\frac{z^{d-1}}{e^{\varphi(z)}} \partial_{z}\left(\frac{e^{\varphi(z)}}{z^{d-1}} \partial_{z}\right)-\partial_{z}^{2} \varphi+\frac{(\mu R)^{2}}{z^{2}}-\frac{d-1}{z^{2}}\right] \Phi_{z}=0 } \\
{\left[\partial_{\mu} \partial^{\mu}-\frac{z^{d-3}}{e^{\varphi(z)}} \partial_{z}\left(\frac{e^{\varphi(z)}}{z^{d-3}} \partial_{z}\right)+\frac{(\mu R)^{2}}{z^{2}}\right] \Phi_{\nu}=-\frac{2}{z} \partial_{\nu} \Phi_{z} . }
\end{aligned}
$$

In the conformal limit $\varphi \rightarrow 0$ we recover the results given in Ref. [175]. In the gauge defined by $\Phi_{z}=0$, the equations (4.10) decouple and we find the wave equation ${ }^{3}$

$$
\left[\partial_{\mu} \partial^{\mu}-\frac{z^{d-3}}{e^{\varphi(z)}} \partial_{z}\left(\frac{e^{\varphi(z)}}{z^{d-3}} \partial_{z}\right)+\left(\frac{\mu R}{z}\right)^{2}\right] \Phi_{\nu}=0 .
$$

A physical spin-1 hadron has physical polarization components $\epsilon_{\nu}(P)$ along the physical coordinates. We thus write

$$
\Phi_{\nu}(x, z)=e^{i P \cdot x} \Phi_{J=1}(z) \epsilon_{\nu}(P)
$$

with invariant mass $P_{\mu} P^{\mu}=M^{2}$. Substituting (4.12) in (4.11) we find the eigenvalue equation

$$
\left[-\frac{z^{d-3}}{e^{\varphi(z)}} \partial_{z}\left(\frac{e^{\varphi(z)}}{z^{d-3}} \partial_{z}\right)+\left(\frac{\mu R}{z}\right)^{2}\right] \Phi_{J=1}=M^{2} \Phi_{J=1}
$$

describing a spin-1 hadronic bound-state.

\subsection{Arbitrary integer spin}

The description of higher-spin fields in pure AdS space is a complex, but relatively well known problem $[83,84,85,86,87,88,89,90]^{4}$. The treatment of higher-spin

\footnotetext{
${ }^{2} \Phi_{z}$ denotes the $d$-th coordinate, $\Phi_{z} \equiv \Phi_{d}$.

${ }^{3}$ Technically we impose the condition $\Phi_{z}=0$ since physical hadrons have no polarization in the $z$ direction. If $\mu=0$ in the Proca action (4.7) this can be viewed as a gauge condition.

${ }^{4}$ The light-front approach can be used advantageously to describe arbitrary spin fields in AdS. See Refs. [86, 89].
} 
states in the "bottom-up" approach to holographic QCD is an important touchstone for this procedure [91], but it requires a simplified and well defined framework to extend the computations to warped spaces ${ }^{5}$ asymptotic to AdS. For example, the approach of Ref. [74] relies on rescaling the solution of a scalar field $\Phi(z), \Phi(z) \rightarrow \Phi_{J}(z)=\left(\frac{z}{R}\right)^{J} \Phi(z)$, thus introducing a spin-dependent factor [74, 176]. The approach of Karch, Katz, Son and Stephanov (KKSS) [61] starts from a gauge-invariant action in a warped AdS space, and uses the gauge invariance of the model to construct explicitly an effective action in terms of higher-spin fields with only the physical degrees of freedom. However, this approach is not applicable to pseudoscalar particles and their trajectories, and their angular excitations do not lead to a relation with light-front quantized QCD, which is the main subject of this report. The treatment described in this report relies on the approach of Ref. [91], which starts from a manifestly covariant action for higher spin states in a warped space asymptotic to AdS.

Fields with integer spin $J$ are represented by a totally symmetric rank- $J$ tensor field $\Phi_{N_{1} \ldots N_{J}}$. Such a symmetric tensor also contains lower spins, which have to be eliminated by imposing subsidiary conditions, as will be discussed below. The action for a spin- $J$ field in $\mathrm{AdS}_{d+1}$ space in the presence of a dilaton background field $\varphi(z)$ is given by

$$
\begin{aligned}
S=\int d^{d} x d z \sqrt{g} e^{\varphi(z)} g^{N_{1} N_{1}^{\prime}} \cdots g^{N_{J} N_{J}^{\prime}}\left(g^{M M^{\prime}} D_{M} \Phi_{N_{1} \ldots N_{J}}^{*} D_{M^{\prime}} \Phi_{N_{1}^{\prime} \ldots N_{J}^{\prime}}\right. \\
\left.-\mu^{2} \Phi_{N_{1} \ldots N_{J}}^{*} \Phi_{N_{1}^{\prime} \ldots N_{J}^{\prime}}+\cdots\right),
\end{aligned}
$$

where $\sqrt{g}=(R / z)^{d+1}$ and $D_{M}$ is the covariant derivative which includes the affine connection (Sec. A.1.1) and $\mu$ is the AdS mass. The omitted terms in the action indicated by $\cdots$, refer to additional terms with different contractions of the indices.

Inserting the covariant derivatives in the action leads to a rather complicated expression. Furthermore the additional terms from different contractions in (4.14) bring an enormous complexity. A considerable simplification in (4.14) is due to the fact that one has only to consider the subspace of tensors $\Phi_{\nu_{1} \nu_{2} \cdots \nu_{J}}$ which has no indices along the $z$-direction. In fact, a physical hadron has polarization indices along the $3+1$ physical coordinates, $\Phi_{\nu_{1} \nu_{2} \cdots \nu_{J}}$, all other components must vanish identically

$$
\Phi_{z N_{2} \cdots N_{J}}=0
$$

\footnotetext{
${ }^{5}$ With warped spaces we denote curved spaces which deviate from AdS spaces either by a dilaton term or by a modified metric.
} 
As will be seen later, the constraints imposed by the mapping of the AdS equations of motion to the LF Hamiltonian in physical space-time for the hadronic bound-state system at fixed LF time, will give further insights in the description of higher spin states, since it allows an explicit distinction between kinematical and dynamical aspects.

As a practical procedure, one starts from an effective action, which includes a $z$ dependent effective AdS mass $\mu_{\text {eff }}(z)$ [91]

$$
\begin{aligned}
S_{e f f}=\int d^{d} x d z \sqrt{g} e^{\varphi(z)} g^{N_{1} N_{1}^{\prime}} \cdots g^{N_{J} N_{J}^{\prime}}\left(g^{M M^{\prime}} D_{M} \Phi_{N_{1} \ldots N_{J}}^{*} D_{M^{\prime}} \Phi_{N_{1}^{\prime} \ldots N_{J}^{\prime}}\right. \\
\left.-\mu_{e f f}^{2}(z) \Phi_{N_{1} \ldots N_{J}}^{*} \Phi_{N_{1}^{\prime} \ldots N_{J}^{\prime}}\right) .
\end{aligned}
$$

Again, for $\varphi \equiv 0$ and a constant mass term $\mu$, the action is AdS invariant. The function $\mu_{\text {eff }}(z)$, which can absorb the contribution from different contractions in (4.14), is $a$ priori unknown. But, as shall be shown below, the additional symmetry breaking due to the $z$-dependence of the effective AdS mass allows a clear separation of kinematical and dynamical effects. In fact, its $z$-dependence can be determined either by the precise mapping of AdS to light-front physics, or by eliminating interference terms between kinematical and dynamical effects [91]. The agreement between the two methods shows how the light-front mapping and the explicit separation of kinematical and dynamical effects are intertwined.

The equations of motion are obtained from the Euler-Lagrange equations in the subspace defined by (4.15)

$$
\frac{\delta S_{e f f}}{\delta \Phi_{\nu_{1} \nu_{2} \cdots \nu_{J}}^{*}}=0
$$

and

$$
\frac{\delta S_{\text {eff }}}{\delta \Phi_{z N_{2} \cdots N_{J}}^{*}}=0 .
$$

The wave equations for hadronic modes follow from the Euler-Lagrange equation (4.17), whereas (4.18) will yield the kinematical constraints required to eliminate the lower-spin states.

The appearance of covariant derivatives in the action for higher spin fields, (4.14) and (4.16), leads to multiple sums and rather complicated expressions. As shown in [91] (See also Appendix D), these expressions simplify considerably if one does not use generally covariant tensors but goes intermediately to a local inertial frame with Lorentz (tangent) indices. The final expression for the equation of motion for AdS fields with all polarizations in the physical directions is derived from (4.17) and one obtains (Sec. 
D.1.1)

$$
\left[\partial_{\mu} \partial^{\mu}-\frac{z^{d-1-2 J}}{e^{\varphi(z)}} \partial_{z}\left(\frac{e^{\varphi(z)}}{z^{d-1-2 J}} \partial_{z}\right)+\frac{(m R)^{2}}{z^{2}}\right] \Phi_{\nu_{1} \ldots \nu_{J}}=0
$$

with

$$
(m R)^{2}=\left(\mu_{e f f}(z) R\right)^{2}-J z \varphi^{\prime}(z)+J(d-J+1),
$$

which agrees with the result found in Refs. [74, 176] by rescaling the wave equation for a scalar field. As will be shown in Chapter 5 mapping to the light front implies that the quantity $m$ is independent of the variable $z$ (See Eq. (5.4)).

Terms in the action which are linear in tensor fields, with one or more indices along the holographic direction, $\Phi_{z N_{2} \cdots N_{J}}$ (See Appendix D), yield from (4.18) the results [91]

$$
\eta^{\mu \nu} \partial_{\mu} \Phi_{\nu \nu_{2} \cdots \nu_{J}}=0, \quad \eta^{\mu \nu} \Phi_{\mu \nu \nu_{3} \cdots \nu_{J}}=0
$$

These are just the kinematical constraints required to eliminate the states with spin lower than $J$ from the symmetric tensors $\Phi_{\nu_{1} \nu_{2} \cdots \nu_{J}}$.

The conditions (4.21) are independent of the conformal symmetry breaking terms $\varphi(z)$ and $\mu(z)$ in the effective action (4.16); they are a consequence of the purely kinematical aspects encoded in the AdS metric. It is remarkable that, although one has started in AdS space with unconstrained symmetric spinors, the non-trivial affine connection of AdS geometry gives precisely the subsidiary conditions needed to eliminate the lower spin states $J-1, J-2, \cdots$ from the fully symmetric AdS tensor field $\Phi_{\nu_{1} \ldots \nu_{J}}$.

In order to make contact with the LF Hamiltonian, one considers hadronic states with momentum $P$ and a $z$-independent spinor $\epsilon_{\nu_{1} \cdots \nu_{J}}(P)$. In holographic QCD such a state is described by a $z$-dependent wave function and a plane wave propagating in physical space-time representing a free hadron

$$
\Phi_{\nu_{1} \cdots \nu_{J}}(x, z)=e^{i P \cdot x} \Phi_{J}(z) \epsilon_{\nu_{1} \cdots \nu_{J}}(P)
$$

with invariant hadron mass $P_{\mu} P^{\mu} \equiv \eta^{\mu \nu} P_{\mu} P_{\nu}=M^{2}$. Inserting (4.22) into the wave equation (4.19) one obtains the bound-state eigenvalue equation

$$
\left[-\frac{z^{d-1-2 J}}{e^{\varphi(z)}} \partial_{z}\left(\frac{e^{\varphi(z)}}{z^{d-1-2 J}} \partial_{z}\right)+\frac{(m R)^{2}}{z^{2}}\right] \Phi_{J}(z)=M^{2} \Phi_{J}(z),
$$


where the normalizable solution of (4.23) is normalized according to

$$
R^{d-1-2 J} \int_{0}^{\infty} \frac{d z}{z^{d-1-2 J}} e^{\varphi(z)} \Phi_{J}^{2}(z)=1
$$

One also recovers from (4.21) and (4.22) the usual kinematical constraints

$$
\eta^{\mu \nu} P_{\mu} \epsilon_{\nu \nu_{2} \cdots \nu_{J}}=0, \quad \eta^{\mu \nu} \epsilon_{\mu \nu \nu_{3} \cdots \nu_{J}}=0
$$

One sees that the wave equation (4.4) and (4.11) for the scalar and vector field, respectively, are special cases of the equation for general spin (4.19) with the mass (4.20). In the case of a scalar field, the covariant derivative is the usual partial derivative, and there are no additional contractions in the action; thus $\mu_{\text {eff }}=\mu=m$ is a constant. For a spin-1 wave equation, there is one additional term from the antisymmetric contraction, and the contribution from the parallel transport cancels out. It is also simple in this case to determine the effective mass $\mu_{\text {eff }}$ in (4.16) by the comparison with the full expression for the action of a vector field which includes the antisymmetric contraction (See Eq. (4.11)). Thus for spin-1, one has $\mu=m$ and $\left(\mu_{\text {eff }}(z) R\right)^{2}=(\mu R)^{2}+z \varphi^{\prime}(z)-d$.

In general, the AdS mass $m$ in the wave equation (4.19) or (4.23) is determined from the mapping to the light-front Hamiltonian (Chaper 5). Since $m$ maps to the Casimir operator of the orbital angular momentum in the light front (a kinematical quantity) it follows that $m$ should be a constant. Consequently, the $z$-dependence of the effective mass (4.20) in the AdS action (4.16) is determined a posteriori by kinematical constraints in the light front, namely by the requirement that the mass $m$ in (4.19) or (4.23) must be a constant.

The relation (4.20) can also be derived independently a priori, if one demands that the kinematical effects from AdS and the dynamical effects due to the breaking of maximal symmetry are clearly separated in the equations of motion [91]. In general, the presence of a dilaton in the effective action (4.16) and the quadratic appearance of covariant derivatives lead to a mixture of kinematical and dynamical effects. However, by choosing the appropriate $z$ dependence of the effective mass $\mu_{\text {eff }}(z)$ the interference terms cancel. This requirement determines $\mu_{\text {eff }}(z)$ completely and one recovers (4.20).

In the case where the maximal symmetry of AdS is not broken by a dilaton, $\varphi(z)=0$, no z-dependent AdS mass is necessary, and one can start with a constant mass term in (4.16). This is the case in the hard-wall model, where dynamical effects are introduced by the boundary conditions and indeed no mixing between kinematical and dynamical 
aspects does occur.

\subsubsection{Confining interaction and warped metrics}

As an alternative to introducing a dilaton term into the action (4.16) in order to break the maximal symmetry of AdS space, the approach presented in this section allows one to equivalently modify the AdS metric by a $J$-independent warp factor

$$
\tilde{g}_{M N}=e^{2 \tilde{\varphi}(z)} g_{M N},
$$

where $g_{M N}$ is the metric tensor of AdS in Poincaré coordinates (Sec. A.2.2). The effective action is then given by

$$
\begin{aligned}
\tilde{S}_{e f f}=\int d^{d} x d z \sqrt{\tilde{g}} \tilde{g}^{N_{1} N_{1}^{\prime}} \ldots \tilde{g}^{N_{J} N_{J}^{\prime}}\left(\tilde{g}^{M M^{\prime}} D_{M} \Phi_{N_{1} \ldots N_{J}}^{*} D_{M^{\prime}} \Phi_{N_{1}^{\prime} \ldots N_{J}^{\prime}}\right. & \\
& \left.-\tilde{\mu}_{e f f}^{2}(z) \Phi_{N_{1} \ldots N_{J}}^{*} \Phi_{N_{1}^{\prime} \ldots N_{J}^{\prime}}\right)
\end{aligned}
$$

where $\sqrt{\tilde{g}}=\left(R e^{\tilde{\varphi}(z)} / z\right)^{d+1}$ and the effective mass $\tilde{\mu}_{\text {eff }}(z)$ is again an a priori unknown function.

The use of warped metrics is useful for visualizing the overall confinement behavior by following an object in warped AdS space as it falls to the infrared region by the effects of gravity. The gravitational potential energy for an object of mass $M$ in general relativity is given in terms of the time-time component of the metric tensor $g_{00}$

$$
V=M c^{2} \sqrt{\tilde{g}_{00}}=M c^{2} R \frac{e^{\tilde{\varphi}(z)}}{z}
$$

thus, one may expect a potential that has a minimum at the hadronic scale $z_{0} \sim 1 / \Lambda_{\mathrm{QCD}}$ and grows fast for larger values of $z$ to confine effectively a particle in a hadron within distances $z \sim z_{0}$. In fact, according to Sonnenschein [177], a background dual to a confining theory should satisfy the conditions for the metric component $g_{00}$

$$
\left.\partial_{z}\left(g_{00}\right)\right|_{z=z_{0}}=0,\left.\quad g_{00}\right|_{z=z_{0}} \neq 0
$$

to display the Wilson loop-area law for confinement of strings.

The action with a warped metric (4.27) and the effective action with a dilaton field (4.16) lead to identical results for the equations of motion for arbitrary spin (4.23), provided that one identifies the metric warp factor $\tilde{\varphi}(z)$ in (4.26) and the effective mass 


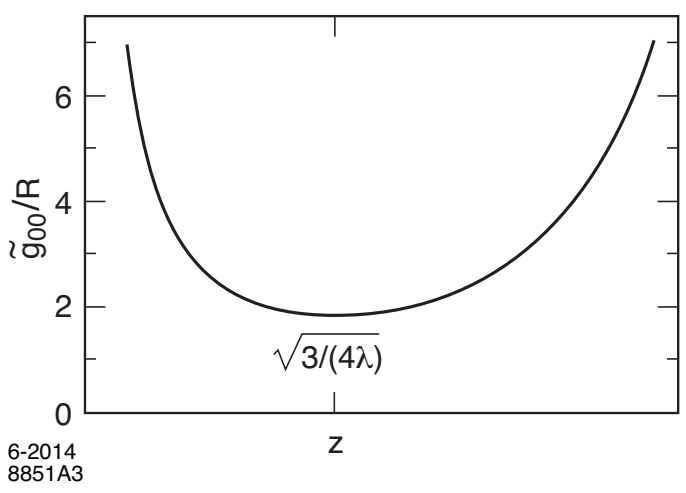

Figure 4.1: $\tilde{g}_{00}(z) / R$ for a positive dilaton profile $\tilde{\varphi}(z)=\lambda z^{2} / 3$.

$\tilde{\mu}_{\text {eff }}(z)$ with the dilaton profile $\varphi(z)$ and the mass $m$ in (4.23) according to

$$
\begin{aligned}
\tilde{\varphi}(z) & =\frac{\varphi(z)}{d-1} \\
\left(\tilde{\mu}_{e f f}(z) R\right)^{2} & =\left(m^{2}+J z \frac{\tilde{\varphi}^{\prime}(z)}{d-1}-J z^{2} \tilde{\Omega}^{2}(z)-J(d-J)\right) e^{-2 \tilde{\varphi}(z)}
\end{aligned}
$$

where $\tilde{\Omega}(z)$ is the warp factor of the affine connection for the metric $(4.26), \tilde{\Omega}(z)=$ $1 / z-\partial_{z} \tilde{\varphi}$ (For more details see Ref. [91] and Sec. D.1.2). As an example, we show in Fig. 4.1 the metric component $\tilde{g}_{00} / R$ for the positive profile $\tilde{\varphi}(z)=\lambda z^{2} / 3$. The corresponding potential (4.28) satisfies Eq. (4.29) which leads to the Wilson loop-area condition for confinement. This type of solution is also expected from simple arguments based on stability considerations, since the potential energy should display a deep minimum as a function of the holographic variable $z$ [178].

\subsubsection{Higher spin in a gauge invariant AdS model}

In their seminal paper [61] Karch et. al., introduced the soft wall model for the treatment of higher spin states in AdS/QCD. They also started from the covariant action (4.14), in $d=4$ dimensions, but in addition demanded that it is invariant under gauge transformations in $\mathrm{AdS}_{5}$

$$
\begin{aligned}
\Phi_{N_{1} \cdots N_{J}} & \rightarrow \Phi_{N_{1} \cdots N_{J}}+\delta \Phi_{N_{1} \cdots N_{J}} \\
\delta \Phi_{N_{1} \cdots N_{J}} & =D_{N_{1}} \xi_{N_{2} \cdots N_{J}}+D_{N_{2}} \xi_{N_{1} \cdots N_{J}}+\cdots
\end{aligned}
$$


where $\xi_{N_{2} \cdots N_{J}}$ is a symmetric tensor of rank $J-1$. In order to achieve gauge invariance, the genuine AdS mass $\mu$ in (4.14) must be $z$-dependent in order to cancel terms in the gauge-transformed action, arising from the affine connection in the covariant derivatives $D_{N}$.

The assumed gauge invariance of the equations of motion allows one to simplify the action (4.14) considerably: one first chooses a gauge in which $\Phi_{z \ldots}=0$. This choice does not fix the gauge uniquely, but there still exist nontrivial tensors $\tilde{\xi}_{N_{2} \cdots N_{J}}$ which leave this condition invariant, i.e., for which

$$
\delta \Phi_{z, \mu_{2} \cdots \mu_{J}}=D_{z} \tilde{\xi}_{N_{2} \cdots N_{J}}+\cdots=0
$$

One can show that invariance of the action under these gauge transformations demands for the rescaled fields

$$
\tilde{\Phi}_{\mu_{1} \cdots \mu_{J}}=\left(\frac{z}{R}\right)^{2 J-2} \Phi_{\mu_{1} \cdots \mu_{J}}
$$

and the following form of the action [61]

$$
S_{\mathrm{KKSS}}=\frac{1}{2} \int d^{4} x d z e^{\lambda z^{2}}\left(\frac{R}{z}\right)^{2 J-1} \eta^{N N^{\prime}} \eta^{\mu_{1} \mu_{1}^{\prime}} \cdots \eta^{\mu_{J} \mu_{J}^{\prime}} \partial_{N} \tilde{\Phi}_{\mu_{1} \cdots \mu_{J}} \partial_{N^{\prime}} \tilde{\Phi}_{\mu_{1}^{\prime} \cdots \mu_{J}^{\prime}}
$$

Applying the variational principle (4.17) and introducing $z$-independent spinors, such as the spinors in (4.22), one obtains the KKSS equation of motion

$$
-\frac{z^{2 J-1}}{e^{\lambda z^{2}}} \partial_{z}\left(\frac{e^{\lambda z^{2}}}{z^{2 J-1}} \tilde{\Phi}_{J}(z)\right)=M^{2} \tilde{\Phi}_{J}(z)
$$

The comparison of (4.36) with (4.23) for $\varphi(z)=\lambda z^{2}$ and $d=4$ shows that the structure is the same, but $m=0$ and the $J$ dependence of the exponents of $z$ are completely different. The phenomenological consequences of the differences, especially concerning the sign of $\lambda$ will be discussed in the next Chapter. In contrast with the method presented in Sec. 4.2.1, the dilaton cannot be absorbed into an additional warp factor in the AdS metric in the treatment based on gauge invariance [61] discussed in this section.

\subsection{Arbitrary half-integer spin}

Fields with half-integer spin, $J=T+\frac{1}{2}$, are conveniently described by RaritaSchwinger spinors [179], $\left[\Psi_{N_{1} \cdots N_{T}}\right]_{\alpha}$, objects which transform as symmetric tensors of 
rank $T$ with indices $N_{1} \ldots N_{T}$, and as Dirac spinors with index $\alpha$. The Lagrangian of fields with arbitrary half-integer spin in a higher-dimensional space is in general more complicated than the integer-spin case. General covariance allows for a superposition of terms of the form

$$
\bar{\Psi}_{N_{1} \ldots N_{T}} \Gamma^{\left[N_{1} \ldots N_{T} M N_{1}^{\prime} \ldots N_{T}^{\prime}\right]} D_{M} \Psi_{N_{1}^{\prime} \ldots N_{T}^{\prime}}
$$

and mass terms

$$
\mu \bar{\Psi}_{N_{1} \ldots N_{T}} \Gamma^{\left[N_{1} \ldots N_{T} N_{1}^{\prime} \ldots N_{T}^{\prime}\right]} \Psi_{N_{1} \ldots N_{T}^{\prime}}
$$

where the tensors $\Gamma^{[\cdots]}$ are antisymmetric products of Dirac matrices and a sum over spinor indices is understood. The maximum number of independent Dirac matrices depends on the dimensionality of space. As a specific example, we present in Sec. D.2.2 the case of $\operatorname{spin} \frac{3}{2}$.

In flat space-time, the equations describing a free particle with spin $T+\frac{1}{2}$ are [179]

$$
\left(i \gamma^{\mu} \partial_{\mu}-M\right) \Psi_{\nu_{1} \cdots \nu_{T}}=0, \quad \gamma^{\nu} \Psi_{\nu \nu_{2} \cdots \nu_{T}}=0
$$

Because of the symmetry of the tensor indices of the spinor $\Psi_{\nu_{1} \cdots \nu_{T}}$ and the anticommutation relation $\gamma^{\mu} \gamma^{\nu}+\gamma^{\nu} \gamma^{\mu}=2 \eta^{\mu \nu}$, the relations in (4.37) imply the subsidiary conditions of the integral spin theory for the $T$ tensor indices (4.21)

$$
\eta^{\mu \nu} \partial_{\mu} \Psi_{\nu \nu_{2} \cdots \nu_{T}}=0, \quad \eta^{\mu \nu} \Psi_{\mu \nu \nu_{3} \cdots \nu_{T}}=0
$$

The actual form of the Dirac equation for Rarita-Schwinger spinors (4.37) in flat space-time motivates us to start with a simple effective action for arbitrary half-integer spin in AdS space which, in the absence of dynamical terms, preserves maximal symmetry of AdS in order to describe the correct kinematics and constraints in physical space-time.

We start with the effective action in $\operatorname{AdS}_{d+1}$

$$
\begin{aligned}
S_{F e f f}=\frac{1}{2} \int d^{d} x d z & \sqrt{g} e^{\varphi(z)} g^{N_{1} N_{1}^{\prime}} \cdots g^{N_{T} N_{T}^{\prime}} \\
& {\left[\bar{\Psi}_{N_{1} \cdots N_{T}}\left(i \Gamma^{A} e_{A}^{M} D_{M}-\mu-\rho(z)\right) \Psi_{N_{1}^{\prime} \cdots N_{T}^{\prime}}+\text { h.c. }\right], }
\end{aligned}
$$

including a dilaton term $\varphi(z)$ and an effective interaction $\rho(z)$ (See also Refs. [180] and [181]). In (4.39) $\sqrt{g}=\left(\frac{R}{z}\right)^{d+1}$ and $e_{A}^{M}$ is the inverse vielbein, $e_{A}^{M}=\left(\frac{z}{R}\right) \delta_{A}^{M}$. The covariant derivative $D_{M}$ of a Rarita-Schwinger spinor includes the affine connection 
and the spin connection (Sec. A.1.3) and the tangent-space Dirac matrices obey the usual anticommutation relation $\left\{\Gamma^{A}, \Gamma^{B}\right\}=2 \eta^{A B}$. For $\varphi(z)=\rho(z)=0$ the effective action (4.39) preserves the maximal symmetry of AdS space. The reason why one has to introduce an additional symmetry breaking term $\rho(z)$ in (4.39) will become clear soon. Similarly to the integer-spin case, where the subsidiary conditions follow from the simple AdS effective action (4.16), it will be shown below that the action (4.39) indeed implies that the Rarita-Schwinger condition given in (4.37), and thus the subsidiary conditions (4.38), follow from the non-trivial kinematics of the higher dimensional gravity theory [91].

Since only physical polarizations orthogonal to the holographic dimension are relevant for fields corresponding to hadron bound states we put

$$
\Psi_{z N_{2} \ldots N_{T}}=0
$$

The equations of motion are derived in a similar way from the effective action (4.39) as in the case of integer spin [91]. Since the covariant derivatives occur only linearly, the expressions are considerably simpler [91]. The equations of motion follow from the variation of the effective action

$$
\frac{\delta S_{F e f f}}{\delta \bar{\Psi}_{\nu_{1} \nu_{2} \cdots \nu_{J}}}=0
$$

and

$$
\frac{\delta S_{F e f f}}{\delta \bar{\Psi}_{z N_{2} \cdots N_{J}}}=0
$$

One then obtains the AdS Dirac-like wave equation (Appendix D)

$$
\left[i\left(z \eta^{M N} \Gamma_{M} \partial_{N}+\frac{d-z \varphi^{\prime}-2 T}{2} \Gamma_{z}\right)-\mu R-R \rho(z)\right] \Psi_{\nu_{1} \ldots \nu_{T}}=0
$$

and the Rarita-Schwinger condition in physical space-time (4.37)

$$
\gamma^{\nu} \Psi_{\nu \nu_{2} \ldots \nu_{T}}=0
$$

Although the dilaton term $\varphi^{\prime}(z)$ shows up in the equation of motion (4.43), it actually does not lead to dynamical effects, since it can be absorbed by rescaling the RaritaSchwinger spinor according to $\Psi_{\nu_{1} \ldots \nu_{T}} \rightarrow e^{\varphi(z) / 2} \Psi_{\nu_{1} \ldots \nu_{T}}$. Thus, from (4.43) one obtains

$$
\left[i\left(z \eta^{M N} \Gamma_{M} \partial_{N}+\frac{d-2 T}{2} \Gamma_{z}\right)-\mu R-R \rho(z)\right] \Psi_{\nu_{1} \ldots \nu_{T}}=0
$$


Therefore, for fermion fields in AdS a dilaton term has no dynamical effects on the spectrum since it can be rotated away [182]. This is a consequence of the linear covariant derivatives in the fermion action, which also prevents a mixing between dynamical and kinematical effects, and thus, in contrast to the effective action for integer spin fields (4.16), the AdS mass $\mu$ in Eq. (4.39) is constant. As a result, one must introduce an effective confining interaction $\rho(z)$ in the fermion action to break conformal symmetry and generate a baryon spectrum $[138,180]$. This interaction can be constrained by the condition that the 'square' of the Dirac equation leads to a potential which matches the dilaton-induced potential for integer spin.

The Rarita-Schwinger condition (4.44) in flat four-dimensional space also entails, with the extended Dirac equation (4.45), the subsidiary conditions for the tensor indices required to eliminate the lower spins [91]. The results from the effective action (4.39) for spin- $\frac{3}{2}$ are in agreement with the results from Refs. [183] and [184] (Sec. D.2.2).

\section{Warped metric}

Identical results for the equations of motion for arbitrary half-integer spin are obtained if one starts with the modified metric (4.26). One finds that the effective fermion action with a dilaton field (4.39) is equivalent to the corresponding fermion action with modified AdS metrics, provided that one identifies the dilaton profile according to $\tilde{\varphi}(z)=\varphi(z) / d$ and the effective mass $\tilde{\mu}(z)$ with the mass $\mu$ in (4.39) according to $\tilde{\mu}(z)=e^{-\tilde{\varphi}(z)} \mu$. Thus, one cannot introduce confinement in the effective AdS action for fermions either by a dilaton profile or by additional warping of the AdS metrics in the infrared. In both cases one requires an additional effective interaction, as introduced in the effective action (4.39), with $\rho(z) \neq 0$. 


\section{Chapter 5}

\section{Light-Front Holographic Mapping and Hadronic Spectrum}

The study of the internal structure and excitation spectrum of mesons and baryons is one of the most challenging aspects of hadronic physics. In fact, an important goal of computations in lattice QCD is the reliable extraction of the excited hadron mass spectrum. Lattice calculations of the ground state of hadron masses agree well with experimental values [185]. However, the excitation spectrum of the light hadrons, and particularly nucleons, represent a formidable challenge to lattice QCD due to the enormous computational complexity required for the extraction of meaningful data beyond the leading ground state configuration [186]. In addition to the presence of continuous thresholds, a large basis of interpolating operators is required since excited hadronic states are classified according to irreducible representations of the lattice, not the total angular momentum. Furthermore, it is not simple to distinguish the different radial excitations by following the propagation of modes in the euclidean lattice because of the identical short distance behavior of radial states. In contrast, the semiclassical light-front holographic wave equation (2.45) describes relativistic bound states at equal light-front time with an analytic simplicity comparable to the Schrödinger equation of atomic physics at equal instant time, where the excitation spectrum follows from the solution of an eigenvalue equation. Also, it is simple to identify the radial excitations in the spectrum as they corresponds to the number of nodes in the eigenfunctions.

The structure of the QCD light-front Hamiltonian (2.7) for the hadron bound state $|\psi(P)\rangle$ formulated at equal light-front time is similar to the structure of the wave equation (4.23) for the $J$-mode $\Phi_{\mu_{1} \cdots \mu_{J}}$ in AdS space; they are both frame independent and have identical eigenvalues $M^{2}$, the mass spectrum of the color-singlet states of QCD. 
This provides the basis for a profound connection between physical QCD formulated in the light front and the physics of hadronic modes in AdS space. However, important differences are also apparent: Eq. (2.7) is a linear quantum-mechanical equation of states in Hilbert space, whereas Eq. (4.23) is a classical gravity equation; its solutions describe spin- $J$ modes propagating in a higher-dimensional warped space. In order to establish a connection between both approaches, it is important to realize that physical hadrons are inexorably endowed with orbital angular momentum since they are composite. Thus, the identification of orbital angular momentum is of primary interest in making such a connection. As we discuss below, this identification follows from the precise mapping between the one-dimensional semiclassical approximation to light-front dynamics found in Chapter 2 and the equations of motion of hadronic spin modes described in Chapter 4. Furthermore, if one imposes the requirement that the action of the corresponding one-dimensional effective theory remains conformal invariant (See Chapter 3), this fixes uniquely the form of the effective potential; and, as we will show below, the dilaton profile to have the specific form $\varphi(z)=\lambda z^{2}$. This remarkable result follows from the dAFF construction of conformally invariant quantum mechanics [94, 95] and the mapping of AdS to light-front physics. The resultant effective theory possess an $S O(2,1)$ algebraic structure, which, as we shall discuss in the present chapter, encodes fundamental dynamical aspects of confinement and reproduces quite well the systematics of the light-hadron excitation spectrum.

In the usual AdS/CFT correspondence the baryon is an $S U\left(N_{C}\right)$ singlet bound state of $N_{C}$ quarks in the large- $N_{C}$ limit. Since there are no quarks in this theory, quarks are introduced as external sources at the AdS asymptotic boundary [37, 187]. The baryon is constructed as an $N_{C}$ baryon vertex located in the interior of AdS. In this top-down string approach baryons are usually described as solitons or Skyrmion-like objects [188, 189, 190]. In contrast, the light-front holographic approach is based on the precise mapping of AdS expressions to the light front in physical space-time. Consequently, we will describe in this review physical baryons corresponding to $N_{C}=3$ not $N_{C} \rightarrow \infty$. In fact, the light-front approach to relativistic bound-state dynamics corresponds to strongly correlated multiple-particle states in the Fock expansion, and we may expect that the large number of degrees of freedom, required to have a valid description in terms of a semiclassical gravity theory, would correspond to the very large number of components in the large $n$-Fock expansion [95]. The enormous complexity arising as a result of the strong interaction dynamics of an infinite number of components and Fock states is encoded in the effective potential $U$. To a first semiclassical approximation, 
this confining potential is determined by the underlying conformal symmetry of the classical QCD Lagrangian incorporated in the effective one-dimensional effective theory. As it turns out, the analytical exploration of the baryon spectrum using gauge/gravity duality ideas is not as simple, or as well understood yet, as the meson case, and further work beyond the scope of the present review is required. However, as we shall discuss below, even a relatively simple approach provides a framework for a useful analytical exploration of the strongly-coupled dynamics of baryons which gives important insights into the systematics of the light-baryon spectrum using simple analytical methods.

\section{$5.1 \quad$ Integer spin}

An essential step is the mapping of the equation of motion describing a hadronic mode in a warped AdS space to the light front. To this end, we compare the relativistic one-dimensional light-front wave equation (2.45) with the spin- $J$ wave equation in AdS (4.23), and factor out the scale $(1 / z)^{J-(d-1) / 2}$ and dilaton factors from the AdS field as follows

$$
\Phi_{J}(z)=\left(\frac{R}{z}\right)^{J-(d-1) / 2} e^{-\varphi(z) / 2} \phi_{J}(z) .
$$

Upon the substitution of the holographic variable $z$ by the light-front invariant variable $\zeta$ and replacing (5.1) into the AdS eigenvalue equation (4.23), we find for $d=4$ the QCD light-front frame-independent wave equation (2.45)

$$
\left(-\frac{d^{2}}{d \zeta^{2}}-\frac{1-4 L^{2}}{4 \zeta^{2}}+U(\zeta)\right) \phi(\zeta)=M^{2} \phi(\zeta)
$$

with the effective potential in the front form of dynamics [191]

$$
U(\zeta, J)=\frac{1}{2} \varphi^{\prime \prime}(\zeta)+\frac{1}{4} \varphi^{\prime}(\zeta)^{2}+\frac{2 J-3}{2 \zeta} \varphi^{\prime}(\zeta)
$$

The AdS mass $m$ in (4.23) is related to the light-front internal orbital angular momentum $L$ and the total angular momentum $J$ of the hadron according to

$$
(m R)^{2}=-(2-J)^{2}+L^{2}
$$

where the critical value $L=0$ corresponds to the lowest possible stable solution. Lightfront holographic mapping thus implies that the AdS mass $m$ in (4.23) is not a free 
parameter but scales according to (5.4), thus giving a precise expression for the AdS effective mass $\mu_{\text {eff }}(z)$ in the AdS effective action (4.16). For $J=0$ the five dimensional AdS mass $m$ is related to the orbital angular momentum of the hadronic bound state by $(m R)^{2}=-4+L^{2}$ and thus $(m R)^{2} \geq-4$. The quantum mechanical stability condition $L^{2} \geq 0$ is thus equivalent to the Breitenlohner-Freedman stability bound in AdS [192].

\subsubsection{A light-front holographic model for mesons}

The simplest holographic example is the truncated model of Polchinski and Strassler whereas the confinement dynamics is included by the boundary conditions at $1 / \Lambda_{\mathrm{QCD}}[55]$. This "hard-wall" model was introduced to study high-energy fixed-angle hard scattering of glueballs using the gauge/gravity duality in confining gauge theories [55]. It was then realized by Boschi-Filho and Braga [193, 194] that this simple model could be used advantageously to compute the glueball mass spectrum and obtain results which compare favorably with more elaborated computations based, for example, on lattice QCD [195, 196] or supergravity in an AdS blackhole geometry background [197, 198, 199] using the AdS/CFT correspondence. The hard-wall model was extended by two of the authors of this report to light hadrons in Ref. [200], where it was shown that the pattern of orbital excitations of light mesons and baryons is well described in terms of a single parameter, the QCD scale $\Lambda_{\mathrm{QCD}}$. In Refs. $[69,70,71]$ it was shown by different authors, including another of the authors of this report, how to construct a five-dimensional holographic model which incorporates chiral symmetry and other properties of light mesons, including quark masses, decay rates and couplings (See Sect. 7.2).

In the light-front version of the hard-wall model [75], the holographic variable $z$ corresponds exactly with the impact variable $\zeta$, which represents the invariant measure of transverse separation of the constituents within the hadrons, and quarks propagate freely in the hadronic interior up to the confinement scale. This model provides an analog of the MIT bag model [201] where quarks are permanently confined inside a finite region of space. In contrast to bag models, boundary conditions are imposed on the boost-invariant variable $\zeta$, not on the bag radius at fixed time. The resulting model is a manifestly Lorentz invariant model with confinement at large distances, while incorporating conformal behavior at small physical separation. The eigenvalues of the LF wave equation (2.45) for the hard-wall model $(U=0)$ are determined by the boundary conditions $\phi\left(z=1 / \Lambda_{\mathrm{QCD}}\right)=0$, and are given in terms of the roots $\beta_{L, k}$ of the Bessel functions: $M_{L, k}=\beta_{L, k} \Lambda_{\mathrm{QCD}}$. By construction, the hard wall model has a simple separation of kinematical and dynamical aspects, but it has shortcomings when trying to 
describe the observed meson spectrum $[200,176]$. The model fails to account for the pion as a chiral $M=0$ state and it is degenerate with respect to the orbital quantum number $L$, thus leading to identical trajectories for pseudoscalar and vector mesons. It also fails to account for the important splitting for the $L=1 \mathrm{a}$-meson states for different values of $J$. Furthermore, for higher quantum excitations the spectrum behaves as $M \sim 2 n+L$, in contrast to the Regge dependence $M^{2} \sim n+L$ found experimentally [202, 203]. As a consequence, the radial modes are not well described in the truncated-space model. For example the first radial AdS eigenvalue has a mass $1.77 \mathrm{GeV}$, which is too high compared to the mass of the observed first radial excitation of the meson, the $\pi(1300)$.

The shortcomings of the hard-wall model are evaded with the "soft-wall" model [61] where the sharp cutoff is modified by a a dilaton profile $\varphi(z) \sim z^{2}$. The soft-wall model leads to linear Regge trajectories [61] and avoids the ambiguities in the choice of boundary conditions at the infrared wall. From the relation (5.3) it follows that the harmonic potential is holographically related to a unique dilaton profile, $\varphi(z)=$ $\lambda z^{2}$ provided that $\varphi(z) \rightarrow 0$ as $z \rightarrow 0$. This choice follows from the requirements of conformal invariance as described in Chapter 3, and leads through (5.3) to the effective LF potential $^{1}$

$$
U(\zeta, J)=\lambda^{2} \zeta^{2}+2 \lambda(J-1)
$$

which corresponds to a transverse oscillator in the light-front. The term $\lambda^{2} \zeta^{2}$ is determined uniquely by the underlying conformal invariance incorporated in the onedimensional effective theory, and the constant term $2 \lambda(J-1)$ by the spin representations in the embedding space.

For the effective potential (5.5) equation (2.45) has eigenfunctions (Appendix G.1)

$$
\phi_{n, L}(\zeta)=|\lambda|^{(1+L) / 2} \sqrt{\frac{2 n !}{(n+L) !}} \zeta^{1 / 2+L} e^{-|\lambda| \zeta^{2} / 2} L_{n}^{L}\left(|\lambda| \zeta^{2}\right)
$$

and eigenvalues

$$
M^{2}=(4 n+2 L+2)|\lambda|+2 \lambda(J-1)
$$

The LF wave functions $\phi(\zeta)=\langle\zeta \mid \phi\rangle$ are normalized as $\langle\phi \mid \phi\rangle=\int d \zeta \phi^{2}(\zeta)=1$ in accordance with (4.24).

Except for $J=1$ the spectrum predictions are significantly different for $\lambda>0$ or

\footnotetext{
${ }^{1}$ The notation $\lambda=\kappa^{2}$ is used in Ref. [176].
} 

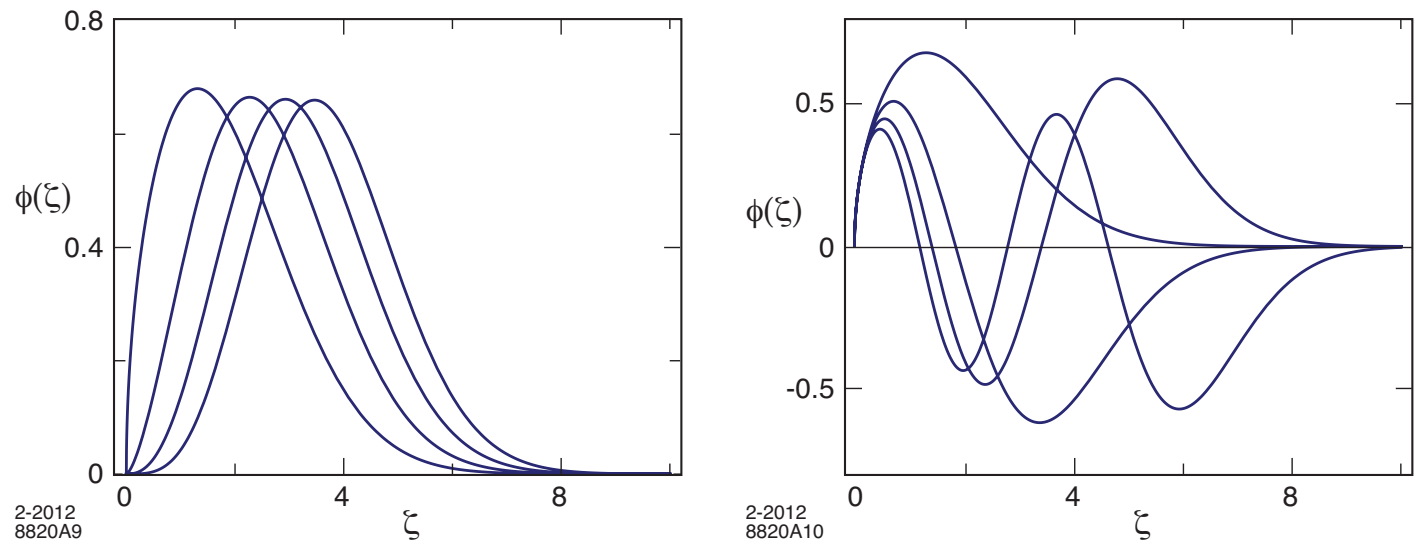

Figure 5.1: Light-front wave functions $\phi_{n, L}(\zeta)$ in physical space-time corresponding to a dilaton profile $\exp \left(\lambda z^{2}\right)$ : (left) orbital modes $(n=0)$ and (right) radial modes $(L=0)$.

$\lambda<0$. The predicted spectrum for $\lambda>0$ is

$$
M_{n, J, L}^{2}=4 \lambda\left(n+\frac{J+L}{2}\right)
$$

For the lowest possible solution $n=L=J=0$ there is an exact cancellation of the LF kinetic and potential energy and the ground state eigenvalue turns out to be $M^{2}=0^{2}$. This is a bound state of two massless quarks and scaling dimension 2, which we identify with the lowest state, the pion. This result not only implies linear Regge trajectories and a massless pion but also the relation between the $\rho$ and $a_{1}$ mass usually obtained from the Weinberg sum rules $[204]^{3}$

$$
m_{\pi}^{2}=M_{0,0,0}^{2}=0, m_{\rho}^{2}=M_{0,1,0}^{2}=2 \lambda, m_{a_{1}}^{2}=M_{0,1,1}^{2}=4 \lambda .
$$

The meson spectrum (5.8) has a string-model Regge form [206]. In fact, the linear dependence of the squared masses in both the angular momentum $L$ and radial quantum number $n, M^{2} \sim n+L$, and thus the degeneracy in the quantum numbers $n+L$, was first predicted using semiclassical quantization of effective strings in Ref. [207]. The LFWFs (5.6) for different orbital and radial excitations are depicted in Fig. 5.1. Constituent quark and antiquark separate from each other as the orbital and radial quantum numbers

\footnotetext{
${ }^{2}$ One can easily write the LF eigenvalue equation for general dimension $d$. The dimension has no influence on the confining term $\lambda^{2} z^{2}$ but it determines the constant term in the potential. For $J=0$ this term is $(2-d) \lambda$. Only in $d=4$ dimensions the vacuum energy is exactly compensated by a constant term in the potential and the $J=0, L=0$ state is massless.

${ }^{3} \mathrm{~A}$ discussion of chiral symmetry breaking in the light-front is given in Ref. [205].
} 
increase. The number of nodes in the light-front wave function depicted in Fig. 5.1 (right) correspond to the radial excitation quantum number $n$. The result (5.8) was also found in Ref. [181].

To compare the LF holographic model predictions with experiment, we list in Table 5.1 confirmed (3-star and 4-star) meson states corresponding to the light-unflavored meson sector from the Particle Data Group (PDG) [16]. The Table includes isospin $I=0$ and $I=1$ vector mesons and the $I=1$ pseudoscalar mesons. We have included the assigned internal spin, orbital angular momentum and radial quantum numbers from the quark content $|u \bar{d}\rangle, \frac{1}{\sqrt{2}}|u \bar{u}-d \bar{d}\rangle$ and $|d \bar{u}\rangle$. The $I=1$ mesons are the $\pi, b, \rho$ and $a$ mesons. We have not listed in Table 5.1 the $I=0$ mesons for the pseudoscalar sector which are a mix of $u \bar{u}, d \bar{d}$ and $s \bar{s}$, thus more complex entities. The light $I=0$ mesons are $\eta, \eta^{\prime}, h, h^{\prime} \omega, \phi, f$ and $f^{\prime}$. This list comprises the puzzling $I=0$ scalar $f$-mesons [208], which may be interpreted as a superposition of tetra-quark states with a $q \bar{q}$ configuration with $L=1, S=1$, which couple to a $J=0$ state [203] ${ }^{4}$. We also include in Table 5.1 the $I=0$ vector-mesons the $f$ and $\omega$, which are well described as a $q \bar{q}$ system with no mixings with the strange sector. Likewise, in Sec. 5.1.3 we compute the masses of the $\phi$ mesons which are well described as an $s \bar{s}$ bound state. We will also discuss in Sec. 5.1.3 light hadronic bound states composed of $u$ or $d$ with an $s$-quark: the $K$ meson and $K^{*}$ vector-meson families.

For the $J=L+S$ meson families Eq. (5.8) becomes

$$
M_{n, L, S}^{2}=4 \lambda\left(n+L+\frac{S}{2}\right) .
$$

The lowest possible stable solution for $n=L=S=0$, the pion, has eigenvalue $M^{2}=0$. Thus one can compute the full $J=L+S$, mass spectrum $M^{2}$ in Table 5.1 , by simply adding $4 \lambda$ for a unit change in the radial quantum number, $4 \lambda$ for a change in one unit in the orbital quantum number and $2 \lambda$ for a change of one unit of spin to the ground state value. The spectral predictions for the $J=L+S$ light unflavored meson states, listed in Table. 5.1, are compared with experimental data in Fig. 5.2 (a) and Fig. 5.3 (a) for the positive sign dilaton model discussed here. The data is from PDG [16].

In contrast to the hard-wall model, the soft-wall model with positive dilaton accounts for the mass pattern observed in radial excitations, as well as for the triplet splitting for the $L=1, J=0,1,2$ observed for the vector meson $a$-states. As we will discuss in the

\footnotetext{
${ }^{4}$ The interpretation of the $\pi_{1}(1400)$ is not very clear [203] and is not included in Table 5.1. Similarly, we do not include the $\pi_{1}(1600)$ in the present analysis.
} 


\begin{tabular}{ccccc}
\hline \hline$L$ & $S$ & $n$ & $J^{P C}$ & Meson State \\
\hline 0 & 0 & 0 & $0^{-+}$ & $\pi(140)$ \\
0 & 0 & 1 & $0^{-+}$ & $\pi(1300)$ \\
0 & 0 & 2 & $0^{-+}$ & $\pi(1800)$ \\
0 & 1 & 0 & $1^{--}$ & $\rho(770)$ \\
0 & 1 & 0 & $1^{--}$ & $\omega(782)$ \\
0 & 1 & 1 & $1^{--}$ & $\omega(1420)$ \\
0 & 1 & 1 & $1^{--}$ & $\rho(1450)$ \\
0 & 1 & 2 & $1^{--}$ & $\omega(1650)$ \\
0 & 1 & 2 & $1^{--}$ & $\rho(1700)$ \\
\hline 1 & 0 & 0 & $1^{+-}$ & $b_{1}(1235)$ \\
1 & 1 & 0 & $0^{++}$ & $a_{0}(980)$ \\
1 & 1 & 1 & $0^{++}$ & $a_{0}(1450)$ \\
1 & 1 & 0 & $1^{++}$ & $a_{1}(1260)$ \\
1 & 1 & 0 & $2^{++}$ & $f_{2}(1270)$ \\
1 & 1 & 0 & $2^{++}$ & $a_{2}(1320)$ \\
1 & 1 & 2 & $2^{++}$ & $f_{2}(1950)$ \\
1 & 1 & 3 & $2^{++}$ & $f_{2}(2300)$ \\
\hline 2 & 0 & 0 & $2^{-+}$ & $\pi_{2}(1670)$ \\
2 & 0 & 1 & $2^{-+}$ & $\pi_{2}(1880)$ \\
2 & 1 & 0 & $3^{--}$ & $\omega_{3}(1670)$ \\
2 & 1 & 0 & $3^{--}$ & $\rho_{3}(1690)$ \\
\hline 3 & 1 & 0 & $4^{++}$ & $a_{4}(2040)$ \\
3 & 1 & 0 & $4^{++}$ & $f_{4}(2050)$ \\
\hline \hline & & & & \\
\hline
\end{tabular}

Table 5.1: Confirmed mesons listed by PDG [16]. The labels $L, S$ and $n$ refer to assigned internal orbital angular momentum, internal spin and radial quantum number respectively. For a $q \bar{q}$ state $P=(-1)^{L+1}, C=(-1)^{L+S}$. For the pseudoscalar sector only the $I=1$ states are listed. 


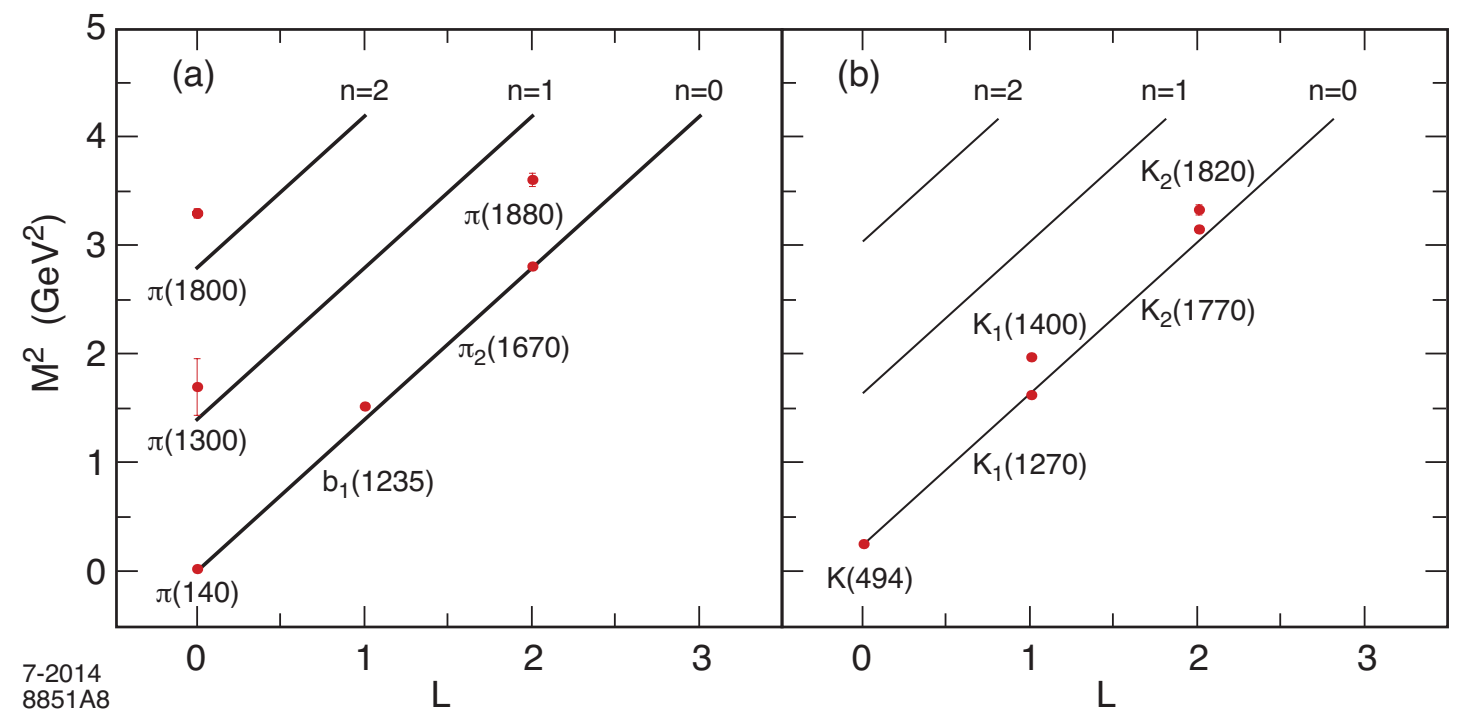

Figure 5.2: Orbital and radial excitation spectrum for the light pseudoscalar mesons: (a) $I=0$ unflavored mesons and (b) strange mesons, for $\sqrt{\lambda}=0.59 \mathrm{GeV}$.

next section, a spin-orbit effect is only predicted for mesons not baryons, as observed in experiment [203, 209]; it thus becomes a crucial test for any model which aims to describe the systematics of the light hadron spectrum. Using the spectral formula (5.8) we find [176]

$$
M_{a_{2}(1320)}>M_{a_{1}(1260)}>M_{a_{0}(980)} .
$$

The predicted values are $0.76,1.08$ and $1.32 \mathrm{GeV}$ for the masses of the $a_{0}(980), a_{1}(1260)$ and $a_{2}(1320)$ vector mesons, compared with the experimental values $0.98,1.23$ and 1.32 $\mathrm{GeV}$ respectively. The prediction for the mass of the $L=1, n=1$ state $a_{0}(1450)$ is 1.53 $\mathrm{GeV}$, compared with the observed value $1.47 \mathrm{GeV}$. Finally, we would like to mention the recent precision measurement at COMPASS [210] which found a new resonance named the $a_{1}(1420)$ with a mass $1.42 \mathrm{GeV}$, the origin of which remains unclear. In the present framework the $a_{1}(1420)$ is interpreted as a $J=1, S=1, L=1, n=1$ vector-meson state with a predicted mass of $1.53 \mathrm{GeV}$. For other calculations of the hadronic spectrum in the framework of AdS/QCD, see Refs. [211, 212, 213, 214, 215, 216, 217, 218, 219, 220, 221, 222, 223, 224, 225, 226, 227, 228, 229, 230, 231, 232, 233, 234, 235, 236, 237, 238, 239] ${ }^{5}$.

The LF holographic model with $\lambda>0$ accounts for the mass pattern observed in the radial and orbital excitations of the light mesons, as well as for the triplet splitting for

\footnotetext{
${ }^{5}$ For recent reviews see, for example, Refs. [114, 240]. One can also use the AdS/QCD framework to study hadrons at finite temperature (See, for example Refs. [241, 242, 243] and references therein) or in a hadronic medium [244].
} 


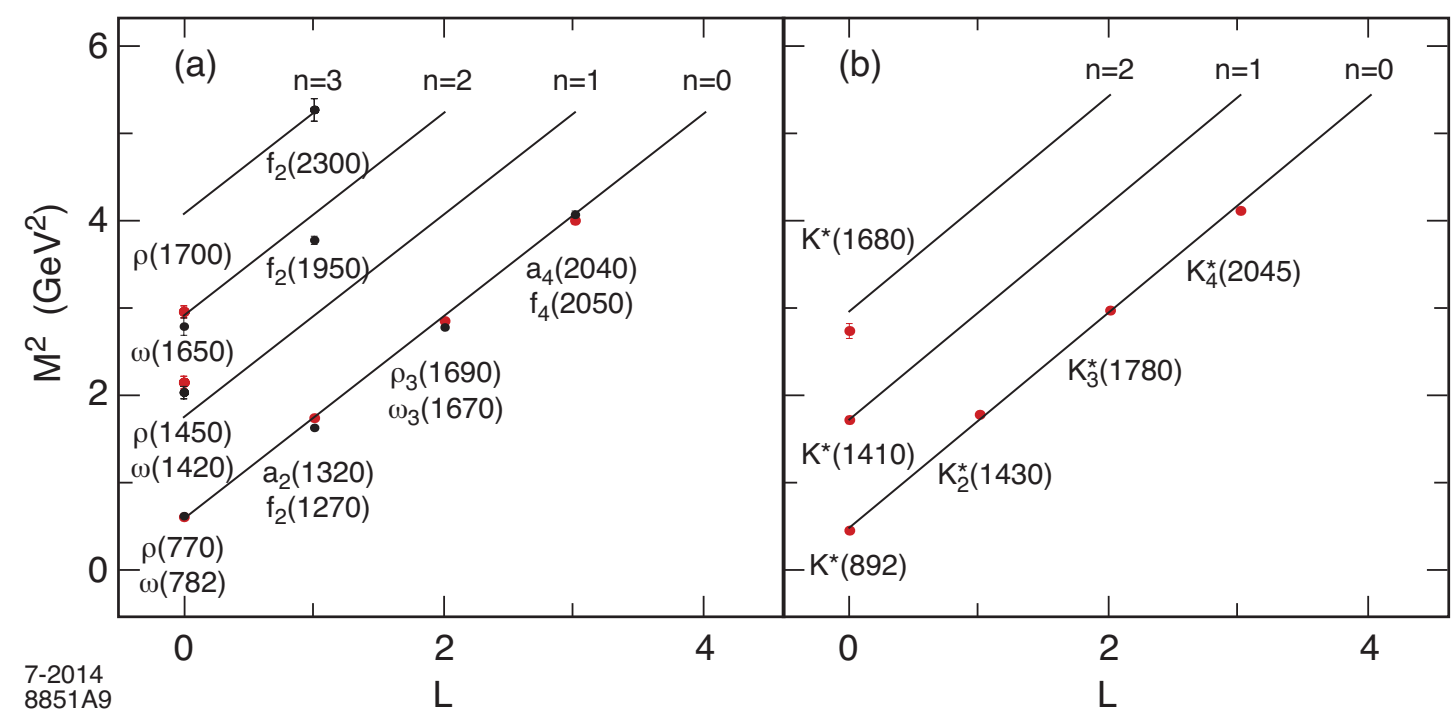

Figure 5.3: Orbital and radial excitation spectrum for the light vector mesons: (a) $I=0$ and $I=1$ unflavored mesons and (b) strange mesons, for $\sqrt{\lambda}=0.54 \mathrm{GeV}$.

the $L=1, J=0,1,2$, vector meson $a$-states [176]. The slope of the Regge trajectories gives a value $\sqrt{\lambda} \simeq 0.5 \mathrm{GeV}$, but the value of $\lambda$ required for describing the pseudoscalar sector is slightly higher that the value of $\lambda$ extracted from the vector sector. In general the description of the vector sector is better than the pseudoscalar sector. However, the prediction for the observed spin-orbit splitting for the $L=1 a$-vector mesons is overestimated by the model.

The solution for $\lambda<0$ leads to a pion mass heavier than the $\rho$ meson and a meson spectrum given by $M^{2}=4 \lambda(n+1+(L-J) / 2$, in clear disagreement with the observed spectrum. Thus the solution $\lambda<0$ is incompatible with the light-front constituent interpretation of hadronic states. We also note that the solution with $\lambda>0$ satisfies the stability requirements from the Wilson loop area condition for confinement [177] discussed in Sec. 4.2.1.

\subsubsection{Meson spectroscopy in a gauge invariant AdS model}

Like the AdS wave equation for arbitrary spin (4.23), the AdS wave equation which follows from a gauge invariant construction described in Sec. 4.2.2 (See Ref. [61]) can be brought into a Schrödinger-like form by rescaling the AdS field in (4.36) according 
to $\tilde{\Phi}_{J}(z)=z^{J-1 / 2} e^{-\lambda z^{2} / 2} \tilde{\phi}_{J}(z)$. The result is

$$
\left(-\frac{d^{2}}{d z^{2}}-\frac{1-4 J^{2}}{4}+\lambda^{2} z^{2}-2 J \lambda\right) \tilde{\phi}_{J}(z)=M^{2} \tilde{\phi}_{J}(z)
$$

and yields the spectrum

$$
M^{2}=(4 n+2 J+2)|\lambda|-2 J \lambda .
$$

Besides the difference in sign in the dilaton profile, there are conceptual differences in the treatment of higher spin given by KKSS [61] in Sec. 4.2.2, as compared with the treatment given in Sec. 4.2. The mapping of the AdS equation of motion (4.36) onto the Schrödinger equation (5.12) reveals that $J=L$ and therefore an essential kinematical degree of freedom is missing in the light-front interpretation of the KKSS AdS wave equation. In particular the $\rho$ meson would be an $L=1$ state. Furthermore the method of treating higher spin, based on gauge invariance, can only be applied to the vector meson trajectory, not pseudoscalar mesons. Generally speaking, one can say that insisting on gauge invariance in $\mathrm{AdS}_{d+1}$ favours a negative dilaton profile $(\lambda<0)$, whereas the mapping onto the LF equation demands an AdS mass $\mu \neq 0$ and a positive profile $(\lambda>0)$.

\subsubsection{Light quark masses and meson spectrum}

In general, the effective interaction depends on quark masses and the longitudinal momentum fraction $x$ in addition to the transverse invariant variable $\zeta$. However, if the confinement potential is unchanged for small quark masses it then only depends on the transverse invariant variable $\zeta$, and the transverse dynamics are unchanged (See Sect. 2.4.1). This is consistent with the fact that the potential is determined from the conformal symmetry of the effective one-dimensional quantum field theory, which is not badly broken for small quark masses.

In the limit of zero quark masses the effective LFWF for a two-parton ground state in impact space is

$$
\psi_{\bar{q} q / \pi}(x, \zeta) \sim \sqrt{x(1-x)} e^{-\frac{1}{2} \lambda \zeta^{2}}
$$

where the invariant transverse variable $\zeta^{2}=x(1-x) \mathbf{b}_{\perp}^{2}$ and $\lambda>0$. The factor $\sqrt{x(1-x)}$ is determined from the precise holographic mapping of transition amplitudes in the limit 
of massless quarks (Chapter 6). The Fourier transform of (5.14) in momentum-space is

$$
\psi_{\bar{q} q / \pi}\left(x, \mathbf{k}_{\perp}\right) \sim \frac{1}{\sqrt{x(1-x)}} e^{-\frac{\mathbf{k}_{\perp}^{2}}{2 \lambda x(1-x)}}
$$

where the explicit dependence of the wave function in the LF off shell-energy is evident.

For the effective two-body bound state the inclusion of light quark masses ${ }^{6}$ amounts to the replacement in (5.15) of the $q-\bar{q}$ invariant mass (2.50), the key dynamical variable which describes the off energy-shell behavior of the bound state [138],

$$
\psi_{\bar{q} q / \pi}\left(x, \mathbf{k}_{\perp}\right) \sim \frac{1}{\sqrt{x(1-x)}} e^{-\frac{1}{2 \lambda}\left(\frac{\mathbf{k}_{\perp}^{2}}{x(1-x)}+\frac{m_{q}^{2}}{x}+\frac{m_{\bar{q}}^{2}}{1-x}\right)},
$$

which has the same exponential form of the succesful phenomenological LFWF introduced in Ref. [245]. The Fourier transform of (5.16) gives the LFWF in impact space including light-quark masses

$$
\psi_{\bar{q} q / \pi}(x, \zeta) \sim \sqrt{x(1-x)} e^{-\frac{1}{2 \lambda}\left(\frac{m_{q}^{2}}{x}+\frac{m_{\bar{q}}^{2}}{1-x}\right)} e^{-\frac{1}{2} \lambda \zeta^{2}}
$$

which factorizes neatly into transverse and longitudinal components. The holographic LFWF (5.17) has been successfully used in the description of diffractive vector meson electroproduction at HERA [246] by extending the LF holographic approach to the longitudinal component of the $\rho \mathrm{LFWF}$, in $B \rightarrow \rho \gamma$ [247] and $B \rightarrow K^{*} \gamma$ [248] decays as well as in the prediction of $B \rightarrow \rho$ [249], $B \rightarrow K^{*}$ form factors [250] and $B \rightarrow K^{*} \mu^{+} \mu^{-}$ decays [251]. This LFWF has also been used to study the spectrum [231] and the distribution amplitudes [252] of light and heavy mesons.

For excited meson states we can follow the same procedure by replacing the key invariant mass variable in the polynomials in the LFWF using (2.50). An explicit calculation shows, however, that the essential modification in the hadronic mass, from small quark masses, comes from the shift in the exponent of the LFWF. The corrections from the shift in the polynomials accounts for less than $3 \%$. This can be understood from the fact that to first order the transverse dynamics is unchanged, and consequently the

\footnotetext{
${ }^{6}$ The light quark masses introduced here are not the constituent masses of the nonrelativistic quark model, but effective quark masses from the reduction of higher Fock states as functionals of the valence state (See Sec. 2.4.1). In the chiral limit, however, these masses should be zero.
} 
transverse LFWF is also unchanged to first order. Thus our expression for the LFWF

$$
\psi_{n, L}(x, \zeta) \sim \sqrt{x(1-x)} e^{-\frac{1}{2 \lambda}\left(\frac{m_{q}^{2}}{x}+\frac{m_{\bar{q}}^{2}}{1-x}\right)} \zeta^{2} e^{-\frac{1}{2} \lambda \zeta^{2}} L_{n}^{L}\left(\lambda \zeta^{2}\right),
$$

and from (2.49) the hadronic mass shift $\Delta M^{2}$ for small quark masses [253]

$$
\Delta M_{m_{q}, m_{\bar{q}}}^{2}=\frac{\int_{0}^{1} d x e^{-\frac{1}{\lambda}\left(\frac{m_{q}^{2}}{x}+\frac{m_{\bar{q}}^{2}}{1-x}\right)}\left(\frac{m_{q}^{2}}{x}+\frac{m_{\bar{q}}^{2}}{1-x}\right)}{\int_{0}^{1} d x e^{-\frac{1}{\lambda}\left(\frac{m_{q}^{2}}{x}+\frac{m_{\bar{q}}^{2}}{1-x}\right)}}
$$

which is independent of $L, S$ and $n$. For light quark masses, the hadronic mass is the longitudinal $1 / x$ average of the square of the effective quark masses, $i$. e., the effective quark masses from the reduction of higher Fock states as functionals of the valence state [161]. The final result for the hadronic spectrum of mesons modified by light quark masses is thus

$$
M_{n, J, L, m_{q}, m_{\bar{q}}}^{2}=\Delta M_{m_{q}, m_{\bar{q}}}^{2}+4 \lambda\left(n+\frac{J+L}{2}\right)
$$

with identical slope $4 \lambda$ from the limit of zero quark masses. In particular, we obtain from (5.20), respectively, the spectral prediction for the unflavored meson and strange meson mass spectrum. We have

$$
M_{n, L, S}^{2}=M_{\pi^{ \pm}}^{2}+4 \lambda\left(n+\frac{J+L}{2}\right)
$$

for the $\pi$ and $b$ pseudoscalar and $\rho, \omega, a, f$ vector mesons, and

$$
M_{n, L, S}^{2}=M_{K^{ \pm}}^{2}+4 \lambda\left(n+\frac{J+L}{2}\right)
$$

for the $K$ and $K^{*}$ meson spectrum. The PDG values are [16] $M_{\pi^{ \pm}} \cong 140 \mathrm{MeV}$ and $M_{K^{ \pm}} \cong 494 \mathrm{MeV}$.

We list in Table 5.2 the confirmed (3-star and 4-star) strange mesons from the Particle Data Group [16]. The predictions for the $J=L+S$ strange pseudoscalar and vector mesons are compared with experimental data in Figs. 5.2 (b) and 5.3 (b) respectively. The data is from PDG [16]. The spectrum is well reproduced with the same values of the mass scale as for the massless case, $\sqrt{\lambda}=0.59 \mathrm{GeV}$ for the light pseudoscalar meson sector and $\sqrt{\lambda}=0.54 \mathrm{GeV}$ for the light vector sector. It is clear 


\begin{tabular}{ccccc}
\hline \hline$L$ & $S$ & $n$ & $J^{P}$ & Meson State \\
\hline 0 & 0 & 0 & $0^{-}$ & $K(494)$ \\
0 & 1 & 0 & $1^{-}$ & $K^{*}(892)$ \\
0 & 1 & 1 & $1^{-}$ & $K^{*}(1410)$ \\
0 & 1 & 2 & $1^{-}$ & $K^{*}(1680)$ \\
\hline 1 & 0 & 0 & $1^{+}$ & $K_{1}(1270)$ \\
1 & 0 & 1 & $1^{+}$ & $K_{1}(1400)$ \\
1 & 1 & 0 & $2^{+}$ & $K_{2}^{*}(1430)$ \\
1 & 1 & 1 & $0^{+}$ & $K_{0}^{*}(1430)$ \\
\hline 2 & 0 & 0 & $2^{-}$ & $K_{2}(1770)$ \\
2 & 0 & 1 & $2^{-}$ & $K_{2}(1820)$ \\
2 & 1 & 0 & $3^{-}$ & $K_{3}^{*}(1780)$ \\
\hline 3 & 1 & 0 & $3^{-}$ & $K_{4}^{*}(2045)$ \\
\hline \hline
\end{tabular}

Table 5.2: Confirmed strange mesons listed by PDG [16]. The labels $L, S$ and $n$ refer to assigned internal orbital angular momentum, internal spin and radial quantum number respectively. For a $q \bar{q}$ state $P=(-1)^{L+1}$. 
from Table 5.2 or Fig. 5.2 (b) that the interpretation of the states $K_{1}(1400)$ and $K_{2}(1820)$ as $n=1$ radial excitations is not straightforward as their masses are very close to the $n=0$ states. As in the case of the light unflavored $q \bar{q}$ mesons, the model predictions are much better for the vector sector. In fact, the model predictions for the $K^{*}$ sector shown in Fig. 5.3 (b) are very good [254]. We note, however, from Table 5.2 that the states $K_{0}^{*}(1430)$ and $K_{2}^{*}(1430)$ - which belong to the $J=0, J=1$ and $J=2$ triplet for $L=1$, are degenerate. This result is in contradiction with the spin-orbit coupling predicted by the LF holographic model for mesons; a possible indication of mixing of the $K_{0}^{*}$ with states which carry the vacuum quantum numbers.

Fitting the quark masses to the observed masses of the $\pi$ and $K$ we obtain for $\sqrt{\lambda}=0.54 \mathrm{MeV}$ the average effective light quark mass $m_{q}=46 \mathrm{MeV}, q=u, d$, and $m_{s}=357 \mathrm{MeV}$, values between the current $\overline{M S}$ Lagrangian masses normalized at $2 \mathrm{GeV}$ and typical constituent masses. With these values one obtains $\Delta M_{m_{q}, m_{\bar{q}}}^{2}=$ $0.067 \lambda, \Delta M_{m_{q}, m_{\bar{s}}}^{2}=0.837 \lambda, \Delta M_{m_{s}, m_{\bar{s}}}^{2}=2.062 \lambda$, for $\sqrt{\lambda}=0.54 \mathrm{GeV}$.

\begin{tabular}{ccccc}
\hline \hline$L$ & $S$ & $n$ & $J^{P}$ & Meson State \\
\hline 0 & 1 & 0 & $1^{-}$ & $\phi(1020)$ \\
0 & 1 & 1 & $1^{-}$ & $\phi(1680)$ \\
0 & 1 & 3 & $1^{-}$ & $\phi(2170)$ \\
\hline 2 & 1 & 0 & $3^{-}$ & $\phi_{3}(1850)$ \\
\hline \hline
\end{tabular}

Table 5.3: Confirmed $\phi$ mesons listed by PDG [16]. The labels $L, S$ and $n$ refer to assigned internal orbital angular momentum, internal spin and radial quantum number respectively. The parity assignment is given by $P=(-1)^{L+1}$.

Since the $\phi$ vector mesons are essentially $s \bar{s}$ bound-states, we can use our previous results to predict the $\phi$ spectrum without introducing any additional parameter. To this end we list in Table 5.3 the confirmed $\phi$ mesons from PDG [16]. The model predictions shown in Fig. 5.4 follow from (5.20) with $\Delta M_{m_{s}, m_{\bar{s}}}^{2}=2.062 \lambda$.

For heavy mesons conformal symmetry is strongly broken and there is no reason to assume that the LF potential in that case is similar to the massless one. Indeed, a simple computation shows that the model predictions for heavy quarks (without introducing additional elements in the model) is not satisfactory. In fact, the data for heavy mesons can only be reproduced at the expense of introducing vastly different values for the scale $\lambda[231,255,256]$. Another important point are the leptonic decay widths. For 


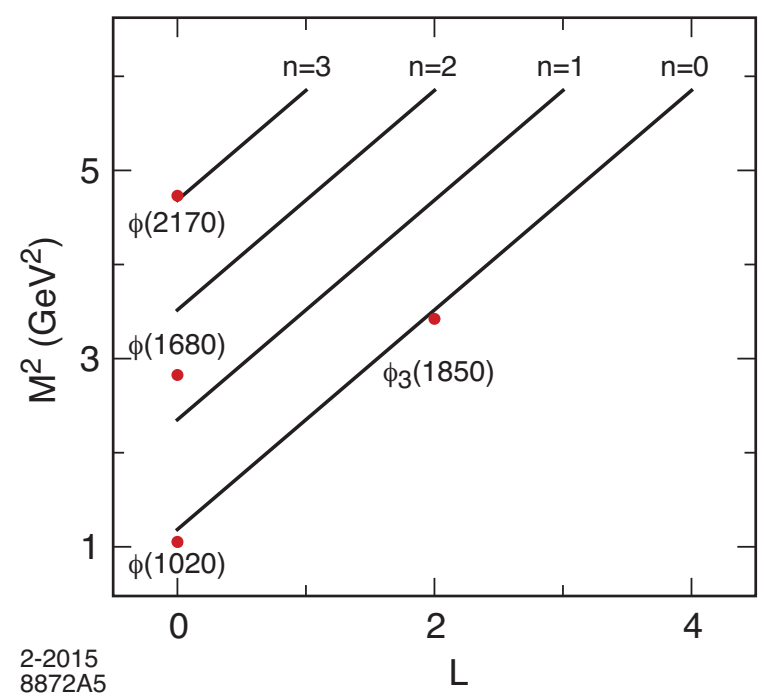

Figure 5.4: Orbital and radial excitation spectrum for the $\phi$ vector mesons for $\sqrt{\lambda}=0.54$ $\mathrm{GeV}$.

light quarks the quark masses have little influence on the result, only about $2 \%$ for the $\pi$ meson and $5 \%$ for the $K$ meson, but using the formalism also for the $B$ and $D$ mesons leads to widely different values when compared with experiment. For large quark masses the form of the LF confinement potential $U$ cannot be obtained from the conformal symmetry of the effective one-dimensional quantum field theory [95]. In this case an important dependence on the heavy quark mass is expected, as suggested by the relation given by Eq. (2.46) between the effective potentials in the front form and instant form of dynamics [97].

\subsection{Half-integer spin}

One can also take as starting point the construction of light-front wave equations in physical space-time for baryons by studying the LF transformation properties of spin- $\frac{1}{2}$ states $[138,257]$. The light-front wave equation describing baryons is a matrix eigenvalue equation $D_{L F}|\psi\rangle=M|\psi\rangle$ with $H_{L F}=D_{L F}^{2}$. In a $2 \times 2$ chiral spinor component representation, the light-front equations are given by the coupled linear differential equations 
(See Appendix H)

$$
\begin{aligned}
-\frac{d}{d \zeta} \psi_{-}-\frac{\nu+\frac{1}{2}}{\zeta} \psi_{-}-V(\zeta) \psi_{-} & =M \psi_{+}, \\
\frac{d}{d \zeta} \psi_{+}-\frac{\nu+\frac{1}{2}}{\zeta} \psi_{+}-V(\zeta) \psi_{+} & =M \psi_{-},
\end{aligned}
$$

where the invariant variable $\zeta$ for an $n$-parton bound state is the transverse impact variable of the $n-1$ spectator system given by (2.47).

As we will consider below, we can identify $\nu$ with the light-front orbital angular momentum $L$, the relative angular momentum between the active and the spectator cluster, but this identification is less straightforward than the relation for mesons, since it involves the internal spin and parity of the 3 -quark baryon configuration. Note that $L$ is the maximal value of $\left|L^{z}\right|$ in a given LF Fock state. An important feature of boundstate relativistic theories is that hadron eigenstates have in general Fock components with different $L$ components. By convention one labels the eigenstate with its minimum value of $L$. For example, the symbol $L$ in the light-front holographic spectral prediction for mesons (5.8) refers to the minimum $L$ (which also corresponds to the leading twist) and $J$ is the total angular momentum of the hadron.

A physical baryon has plane-wave solutions with four-momentum $P_{\mu}$, invariant mass $P_{\mu} P^{\mu}=M^{2}$, and polarization indices along the physical coordinates. It thus satisfies the Rarita-Schwinger equation for spinors in physical space-time (4.37)

$$
\left(i \gamma^{\mu} \partial_{\mu}-M\right) u_{\nu_{1} \cdots \nu_{T}}(P)=0, \quad \gamma^{\nu} u_{\nu \nu_{2} \cdots \nu_{T}}(P)=0 .
$$

Factoring out from the AdS spinor field $\Psi$ the four-dimensional plane-wave and spinor dependence, as well as the scale factor $(1 / z)^{T-d / 2}$, we write

$$
\Psi_{\nu_{1} \cdots \nu_{T}}^{ \pm}(x, z)=e^{i P \cdot x} u_{\nu_{1} \cdots \nu_{T}}^{ \pm}(P)\left(\frac{R}{z}\right)^{T-d / 2} \psi_{T}^{ \pm}(z),
$$

where $T=J-\frac{1}{2}$ and the chiral spinor $u_{\nu_{1} \ldots \nu_{T}}^{ \pm}=\frac{1}{2}\left(1 \pm \gamma_{5}\right) u_{\nu_{1} \ldots \nu_{T}}$ satisfies the fourdimensional chirality equations

$$
\gamma \cdot P u_{\nu_{1} \ldots \nu_{T}}^{ \pm}(P)=M u_{\nu_{1} \ldots \nu_{T}}^{\mp}(P), \quad \gamma_{5} u_{\nu_{1} \ldots \nu_{T}}^{ \pm}(P)= \pm u_{\nu_{1} \ldots \nu_{T}}^{ \pm}(P) .
$$

Upon replacing the holographic variable $z$ by the light-front invariant variable $\zeta$ and 
substituting (5.25) into the AdS wave equation (4.45) for arbitrary spin $J$, we recover the LF expression (5.23), provided that $|\mu R|=\nu+\frac{1}{2}$ and $\psi_{T}^{ \pm}=\psi_{ \pm}$, independent of the value of $J=T+\frac{1}{2}$. We also find that the effective LF potential in the light-front Dirac equation (5.23) is determined by the effective interaction $\rho(z)$ in the effective action $(4.39)$,

$$
V(\zeta)=\frac{R}{\zeta} \rho(\zeta)
$$

which is a $J$-independent potential. This is a remarkable result, since it implies that independently of the specific form of the potential, the value of the baryon masses along a given Regge trajectory depends only on the LF orbital angular momentum $L$, and thus, in contrast with the vector mesons, there is no spin-orbit coupling, in agreement with the observed near-degeneracy in the baryon spectrum [203, 209]. Equation (5.23) is equivalent to the system of second order equations

$$
\left(-\frac{d^{2}}{d \zeta^{2}}-\frac{1-4 \nu^{2}}{4 \zeta^{2}}+U^{+}(\zeta)\right) \psi_{+}=\mathcal{M}^{2} \psi_{+},
$$

and

$$
\left(-\frac{d^{2}}{d \zeta^{2}}-\frac{1-4(\nu+1)^{2}}{4 \zeta^{2}}+U^{-}(\zeta)\right) \psi_{-}=\mathcal{M}^{2} \psi_{-}
$$

where

$$
U^{ \pm}(\zeta)=V^{2}(\zeta) \pm V^{\prime}(z)+\frac{1+2 \nu}{\zeta} V(\zeta)
$$

\subsubsection{A light-front holographic model for baryons}

As for the case of mesons, the simplest holographic model of baryons is the hardwall model, where confinement dynamics is introduced by the boundary conditions at $z \simeq 1 / \Lambda_{\mathrm{QCD}}$. To determine the boundary conditions we integrate (4.39) by parts for $\varphi(z)=\rho(z)=0$ and use the equations of motion. We then find

$$
S_{F}=-\left.\lim _{\epsilon \rightarrow 0} R^{d} \int \frac{d^{d} x}{2 z^{d}}\left(\bar{\Psi}_{+} \Psi_{-}-\bar{\Psi}_{-} \Psi_{+}\right)\right|_{\epsilon} ^{z_{0}}
$$

where $\Psi_{ \pm}=\frac{1}{2}\left(1 \pm \gamma_{5}\right) \Psi$. Thus in a truncated-space holographic model, the light-front modes $\Psi_{+}$or $\Psi_{-}$should vanish at the boundary $z=0$ and $z_{0}=1 / \Lambda_{\mathrm{QCD}}$. This condition fixes the boundary conditions and determines the baryon spectrum in the truncated hardwall model [200]: $M^{+}=\beta_{\nu, k} \Lambda_{\mathrm{QCD}}$, and $M^{-}=\beta_{\nu+1, k} \Lambda_{\mathrm{QCD}}$, with a scale-independent mass ratio determined by the zeros of Bessel functions $\beta_{\nu, k}$. Equivalent results follow 
from the hermiticity of the LF Dirac operator $D_{L F}$ in the eigenvalue equation $D_{L F}|\psi\rangle=$ $\mathcal{M}|\psi\rangle$. The orbital excitations of baryons in this model are approximately aligned along two trajectories corresponding to even and odd parity states [176, 200]. The spectrum shows a clustering of states with the same orbital $L$, consistent with a strongly suppressed spin-orbit force. As for the case for mesons, the hard-wall model predicts $M \sim 2 n+L$, in contrast to the usual Regge behavior $M^{2} \sim n+L$ found experimentally [203, 209]. The radial modes are also not well described in the truncated-space model.

Let us now examine a model similar to the soft-wall dilaton model for mesons by introducing an effective potential, which also leads to linear Regge trajectories in both the orbital and radial quantum numbers for baryon excited states. As we have discussed in Sec. 4.3, a dilaton factor in the fermion action can be scaled away by a field redefinition. We thus choose instead an effective linear confining potential $V=\lambda_{F} \zeta$ which reproduces the linear Regge behavior for baryons $[138,180]$. Choosing $V=\lambda_{F} \zeta$ we find from (5.30)

$$
\begin{aligned}
& U^{+}(\zeta)=\lambda_{F}^{2} \zeta^{2}+2(\nu+1) \lambda_{F}, \\
& U^{-}(\zeta)=\lambda_{F}^{2} \zeta^{2}+2 \nu \lambda_{F}
\end{aligned}
$$

and from (5.28) and (5.29) the two-component solution

$$
\begin{aligned}
& \psi_{+}(\zeta) \sim \zeta^{\frac{1}{2}+\nu} e^{-\left|\lambda_{F}\right| \zeta^{2} / 2} L_{n}^{\nu}\left(\left|\lambda_{F}\right| \zeta^{2}\right) \\
& \psi_{-}(\zeta) \sim \zeta^{\frac{3}{2}+\nu} e^{-\left|\lambda_{F}\right| \zeta^{2} / 2} L_{n}^{\nu+1}\left(\left|\lambda_{F}\right| \zeta^{2}\right)
\end{aligned}
$$

We can compute separately the eigenvalues for the wave equations (5.28) and (5.29) for arbitrary $\lambda_{F}$ and compare the results for consistency, since the eigenvalues determined from both equations should be identical. For the potential (5.32) the eigenvalues of (5.28) are

$$
M_{+}^{2}=(4 n+2 \nu+2)\left|\lambda_{F}\right|+2(\nu+1) \lambda_{F},
$$

whereas for the potential (5.33) the eigenvalues of (5.29) are

$$
M_{-}^{2}=(4 n+2(\nu+1)+2)\left|\lambda_{F}\right|+2 \nu \lambda_{F} .
$$

For $\lambda_{F}>0$ we find $M_{+}^{2}=M_{-}^{2}=M^{2}$ where

$$
M^{2}=4 \lambda_{F}(n+\nu+1),
$$


identical for plus and minus eigenfunctions. For $\lambda_{F}<0$ it follows that $M_{+}^{2} \neq M_{-}^{2}$ and no solution is possible. Thus the solution $\lambda_{F}<0$ is discarded. Note that, as expected, the oscillator form $\lambda_{F}^{2} \zeta^{2}$ in the second order equations (5.28) and (5.29), matches the softwall potential for mesons prescribed by the underlying conformality of the classical QCD Lagrangian as discussed in Chapter 3. We thus set $\lambda_{F}=\lambda$ reproducing the universality of the Regge slope for mesons and baryons. Notice that in contrast with the meson spectrum (5.8), the baryon spectrum (5.38) in the soft wall does not depend on $J$, an important result also found in Ref. [181].

It is important to notice that the solutions (5.34) and (5.35) of the second-order differential equations (5.28) and (5.29) are not independent since the solutions must also obey the linear Dirac equation (5.23) [258]. This fixes the relative normalization. Using the relation $L_{n-1}^{\nu+1}(x)+L_{n}^{\nu}(x)=L_{n}^{\nu+1}(x)$ between the associated Laguerre functions we find for $\lambda=\lambda_{F}>0$

$$
\begin{aligned}
& \psi_{+}(\zeta)=\lambda^{(1+\nu) / 2} \sqrt{\frac{2 n !}{(n+\nu-1) !}} \zeta^{\frac{1}{2}+\nu} e^{-\lambda \zeta^{2} / 2} L_{n}^{\nu}\left(\lambda \zeta^{2}\right), \\
& \psi_{-}(\zeta)=\lambda^{(2+\nu) / 2} \frac{1}{\sqrt{n+\nu+1}} \sqrt{\frac{2 n !}{(n+\nu-1) !}} \zeta^{\frac{3}{2}+\nu} e^{-\lambda \zeta^{2} / 2} L_{n}^{\nu+1}\left(\lambda \zeta^{2}\right),
\end{aligned}
$$

with equal probability

$$
\int d \zeta \psi_{+}^{2}(\zeta)=\int d \zeta \psi_{-}^{2}(\zeta)=1
$$

Equation (5.41) implies that the spin $S_{q}^{z}$ of the quark in the proton has equal probability to be aligned or anti-aligned with the proton's spin $J^{z}$. Thus there is equal probability for states with $L_{q}^{z}=0$ and $L_{q}^{z}= \pm 1$. This remarkable equality means that in the chiral limit the proton's spin $J^{z}$ is carried by the quark orbital angular momentum: $J^{z}=\left\langle L_{q}^{z}\right\rangle= \pm 1 / 2$. This equality also holds for the hard-wall model.

We list in Table 5.4 the confirmed (3-star and 4-star) baryon states from updated Particle Data Group [16] ${ }^{7}$. To determine the internal spin, internal orbital angular momentum and radial quantum number assignment of the $N$ and $\Delta$ excitation spectrum from the total angular momentum-parity PDG assignment, it is convenient to use the conventional $S U(6) \supset S U(3)_{\text {flavor }} \times S U(2)_{\text {spin }}$ multiplet structure [260], but other model choices are also possible $[209]^{8}$.

\footnotetext{
${ }^{7} \mathrm{~A}$ recent exploration of the properties of baryon resonances derived from a multichannel partial wave analysis [259] report additional resonances not included in the Review of Particle Properties [16].

${ }^{8}$ In particular the $\Delta \frac{5}{2}^{-}(1930)$ state (also shown in Table 5.4) has been given the non- $S U(6)$ assign-
} 


\begin{tabular}{|c|c|c|c|c|}
\hline$S U(6)$ & $S$ & $L$ & $n$ & Baryon State \\
\hline \multirow[t]{2}{*}{56} & $\frac{1}{2}$ & 0 & 0 & $N \frac{1}{2}^{+}(940)$ \\
\hline & $\frac{3}{2}$ & 0 & 0 & $\Delta_{2^{3}}^{+}(1232)$ \\
\hline \multirow[t]{2}{*}{56} & $\frac{1}{2}$ & 0 & 1 & $N \frac{1}{2}^{+}(1440)$ \\
\hline & $\frac{3}{2}$ & 0 & 1 & $\Delta \frac{3}{2}^{+}(1600)$ \\
\hline \multirow[t]{3}{*}{70} & $\frac{1}{2}$ & 1 & 0 & $N \frac{1}{2}^{-}(1535) \quad N \frac{3}{2}^{-}(1520)$ \\
\hline & $\frac{3}{2}$ & 1 & 0 & $N \frac{1}{2}^{-}(1650) \quad N \frac{3}{2}^{-}(1700) \quad N \frac{5}{2}^{-}(1675)$ \\
\hline & $\frac{1}{2}$ & 1 & 0 & $\Delta \frac{1}{2}^{-}(1620) \quad \Delta_{\frac{3}{2}^{-}}^{-}(1700)$ \\
\hline \multirow[t]{3}{*}{56} & $\frac{1}{2}$ & 0 & 2 & $N \frac{1}{2}^{+}(1710)$ \\
\hline & $\frac{1}{2}$ & 2 & 0 & $N_{\frac{3}{2}^{+}}{ }^{+}(1720) \quad \mathrm{N}^{\frac{5}{2}}{ }^{+}(1680)$ \\
\hline & $\frac{3}{2}$ & 2 & 0 & $\Delta \frac{1}{2}^{+}(1910) \quad \Delta_{\frac{3}{2}}^{+}(1920) \quad \Delta_{\frac{5}{2}}^{+}(1905) \quad \Delta_{\frac{7}{2}}^{+}(1950)$ \\
\hline \multirow[t]{2}{*}{70} & $\frac{3}{2}$ & 1 & 1 & $N \frac{3}{2}^{-}(1875) \quad N \frac{5}{2}^{-}$ \\
\hline & $\frac{3}{2}$ & 1 & 1 & $\Delta \frac{5}{2}^{-}(1930)$ \\
\hline 56 & $\frac{1}{2}$ & 2 & 1 & $N \frac{3}{2}^{+}(1900) \quad N \frac{5}{2}^{+}$ \\
\hline \multirow[t]{3}{*}{70} & $\frac{1}{2}$ & 3 & 0 & $N \frac{5}{2}^{-} \quad N \frac{7}{2}^{-}$ \\
\hline & $\frac{3}{2}$ & 3 & 0 & $N \frac{5}{2}^{-} \quad N \frac{7}{2}^{-}(2190) \quad N \frac{9}{2}^{-}(2250)$ \\
\hline & $\frac{1}{2}$ & 3 & 0 & $\Delta \frac{5}{2}^{-} \quad \Delta \frac{7}{2}^{-}$ \\
\hline \multirow[t]{2}{*}{56} & $\frac{1}{2}$ & 4 & 0 & $N \frac{9}{2}^{+}(2220)$ \\
\hline & $\frac{3}{2}$ & 4 & 0 & $\Delta \frac{7}{2}^{+} \quad \Delta \frac{9}{2}^{+} \quad \Delta \frac{11}{2}^{+}(2420)$ \\
\hline \multirow[t]{2}{*}{70} & $\frac{1}{2}$ & 5 & 0 & $N \frac{11}{2}^{-}$ \\
\hline & $\frac{3}{2}$ & 5 & 0 & $N \frac{9}{2}^{-} \quad N \frac{11}{2}^{-}(2600) \quad N \frac{13}{2}^{-}$ \\
\hline
\end{tabular}

Table 5.4: Classification of confirmed baryons listed by the PDG [16]. The labels $L, S$ and $n$ refer to the internal orbital angular momentum, internal spin and radial quantum number respectively. The even-parity baryons correspond to the $\mathbf{5 6}$ multiplet of $S U(6)$ and the oddparity to the $\mathbf{7 0}$. 

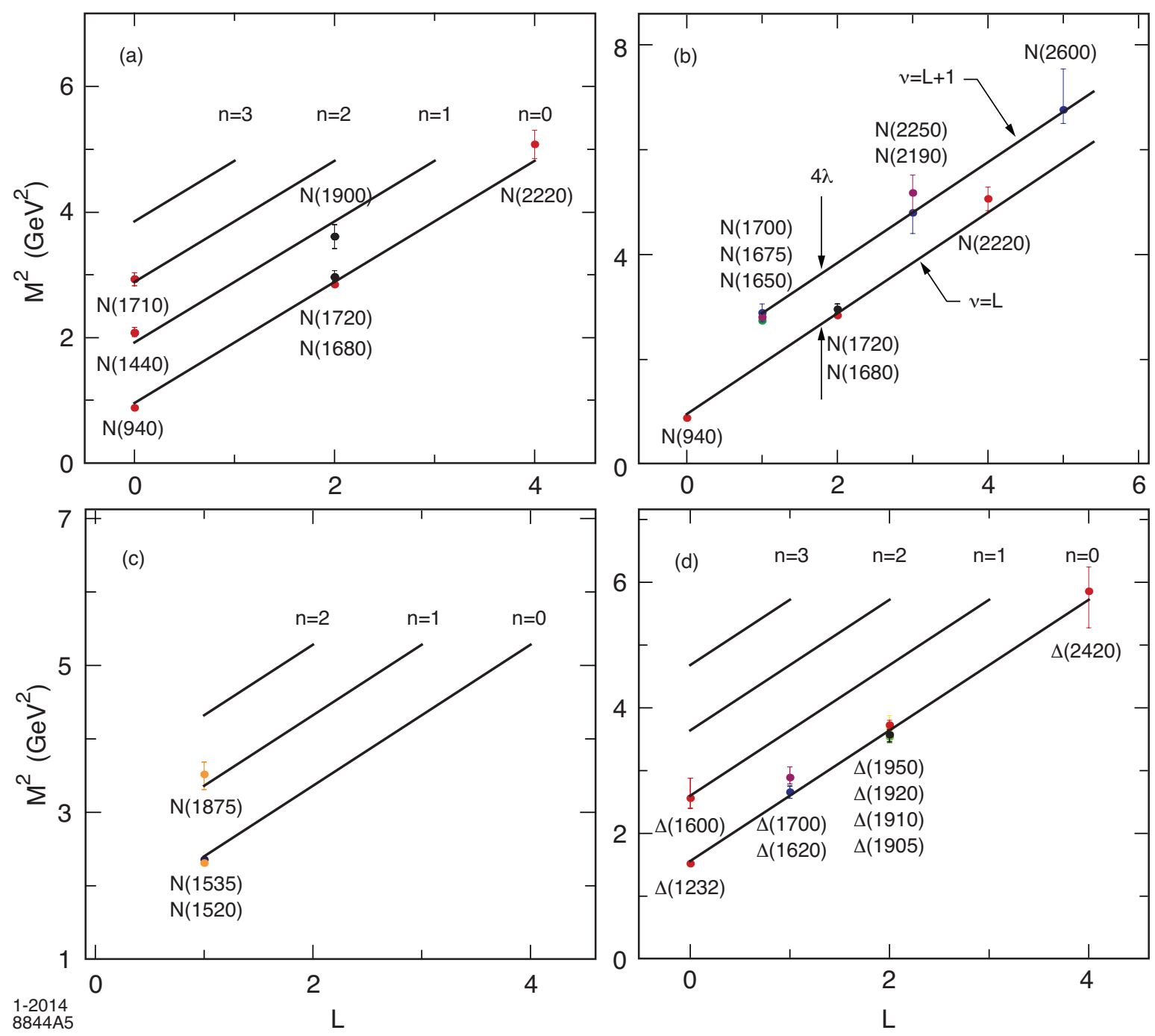

Figure 5.5: Orbital and radial baryon excitation spectrum. Positive-parity spin- $\frac{1}{2}$ nucleons (a) and spectrum gap between the negative-parity spin- $\frac{3}{2}$ and the positive-parity spin- $\frac{1}{2}$ nucleons families (b). Negative parity $N$ (c) and positive and negative parity $\Delta$ families (d). The values of $\sqrt{\lambda}$ are $\sqrt{\lambda}=0.49 \mathrm{GeV}$ (nucleons) and $0.51 \mathrm{GeV}$ (deltas). 
As for the case of mesons, our first task is to identify the lowest possible stable state, the proton, which corresponds to $n=0$ and $\nu=0$. This fixes the scale $\sqrt{\lambda} \simeq 0.5$ $\mathrm{GeV}$. The resulting predictions for the spectroscopy of the positive-parity spin- $\frac{1}{2}$ light nucleons are shown in Fig. 5.5 (a) for the parent Regge trajectory for $n=0$ and $\nu=0,2,4, \cdots, L$, where $L$ is the relative LF angular momentum between the active quark and the spectator cluster. The predictions for the daughter trajectories for $n=1$, $n=2, \cdots$ are also shown in this figure. Only confirmed PDG [16] states are shown. The Roper state $N(1440)$ and the $N(1710)$ are well accounted for in this model as the first and second radial excited states of the proton. The newly identified state, the $N(1900)$ [16] is depicted here as the first radial excitation of the $N(1720)$. The model is successful in explaining the parity degeneracy observed in the light baryon spectrum, such as the $L=2, N(1680)-N(1720)$ degenerate pair in Fig. 5.5 (a).

In Fig. 5.5 (b) we compare the positive parity spin- $\frac{1}{2}$ parent nucleon trajectory with the negative parity spin- $\frac{3}{2}$ nucleon trajectory. It is remarkable that the gap scale $4 \lambda$ determines not only the slope of the trajectories, but also the spectrum gap between the positive-parity spin- $\frac{1}{2}$ and the negative-parity spin- $\frac{3}{2}$ nucleon families, as indicated by arrows in this figure. This means the respective assignment $\nu=L$ and $\nu=L+1$ for the lower and upper trajectories in Fig. 5.5 (b). We also note that the degeneracy of states with the same orbital quantum number $L$ is also well described, as for example the degeneracy of the $L=1$ states $N(1650), N(1675)$ and $N(1700)$ in Fig. 5.5 (b).

We have also to take into account baryons with negative parity and internal spin $S=\frac{1}{2}$, such as the $N(1535)$, as well as baryon states with positive parity and internal spin $S=\frac{3}{2}$, such as the $\Delta(1232)$. Those states are well described by the assignment $\nu=L+\frac{1}{2}$. This means, for example, that $M_{n, L, S=\frac{3}{2}}^{2(+)}=M_{n, L, S=\frac{1}{2}}^{2(-)}$ and consequently the positive and negative-parity $\Delta$ states lie in the same trajectory consistent with the experimental results, as depicted in Fig. 5.5 (d). The newly found state, the $N(1875)$ [16], depicted in Fig. 5.5 (c) is well described as the first radial excitation of the $N(1520)$, and the near degeneracy of the $N(1520)$ and $N(1535)$ is also well accounted. Likewise, the $\Delta(1660)$ corresponds to the first radial excitation of the $\Delta(1232)$ as shown in Fig. 5.5 (d). The model explains the important degeneracy of the $L=2, \Delta(1905), \Delta(1910)$, $\Delta(1920), \Delta(1950)$ states which are degenerate within error bars. The parity degeneracy of the light baryons is also a property of the hard-wall model, but in that case the radial states are not well described [200]. Our results for the $\Delta$ states agree with those of Ref. [216]. "Chiral partners" [261] such as the $N(1535)$ and the $N(940)$ with the same

ment $S=3 / 2, L=1, n=1$ in Ref. [209]. This assignment will be further discussed below. 
total angular momentum $J=\frac{1}{2}$, but with different orbital angular momentum are nondegenerate from the onset ${ }^{9}$. To recapitulate, the parameter $\nu$, which is related to the fifth dimensional AdS mass by the relation $|\mu R|=\nu+1$, has the internal spin $S$ and parity $P$ assignment given in Table 5.5 shown below [262].

\begin{tabular}{c|cc} 
& $S=\frac{1}{2}$ & $S=\frac{3}{2}$ \\
\hline $\mathrm{P}=+$ & $\nu=L$ & $\nu=L+\frac{1}{2}$ \\
$\mathrm{P}=-$ & $\nu=L+\frac{1}{2}$ & $\nu=L+1$ \\
\hline
\end{tabular}

Table 5.5: Orbital assignment for baryon trajectories according to parity and internal spin.

The assignment $\nu=L$ for the lowest trajectory, the proton trajectory, is straightforward and follows from the mapping of AdS to light-front physics. The assignment for other spin and parity baryons states given in Table 5.5 is phenomenological. It is expected that further analysis of the different quark configurations and symmetries of the baryon wave function, as suggested by the model discussed in Ref. [221], will indeed explain the actual assignment given in this table. This particular assignment successfully describes the full light baryon orbital and radial excitation spectrum, and in particular the gap between trajectories with different parity and internal spin [262]. If we follow the non- $S U(6)$ quantum number assignment for the $\Delta(1930)$ given in Ref. [209], namely $S=3 / 2, L=1, n=1$ we find the value $\mathcal{M}_{\Delta(1930)}=4 \sqrt{\lambda} \simeq 2 \mathrm{GeV}$, consistent with the experimental result $1.96 \mathrm{GeV}[16]$.

An important feature of light-front holography is that it predicts a similar multiplicity of states for mesons and baryons, consistent with what is observed experimentally [203]. This remarkable property could have a simple explanation in the cluster decomposition of the holographic variable (2.47), which labels a system of partons as an active quark plus a system of $n-1$ spectators. From this perspective, a baryon with $n=3$ looks in light-front holography as a quark-diquark system. It is also interesting to notice that in the hard-wall model the proton mass is entirely due to the kinetic energy of the light quarks, whereas in the soft-wall model described here, half of the invariant mass squared $M^{2}$ of the proton is due to the kinetic energy of the partons, and half is due to the confinement potential.

\footnotetext{
${ }^{9}$ Since our approach is based on a semiclassical framework, the Regge trajectories remain linear and there is no chiral symmetry restoration for highly excited states [62].
} 


\section{Chapter 6}

\section{Light-Front Holographic Mapping and Transition Amplitudes}

A form factor in QCD is defined by the transition matrix element of a local quark current between hadronic states. The great advantage of the front form - as emphasized in Chapter 2 - is that boost operators are kinematical. Unlike in the instant form, the boost operators in the front form have no interaction terms. The calculation of a current matrix element $\left\langle P+q\left|J^{\mu}\right| P\right\rangle$ requires boosting the hadronic eigenstate from $|P\rangle$ to $|P+q\rangle$, a task which becomes hopelessly complicated in the instant form which includes changes even in particle number for the boosted state [263, 264]. In fact, the boost of a composite system at fixed time $x^{0}$ is only known at weak binding $[265,266]$. In addition, the virtual photon couples to connected currents which arise from the instantform vacuum.

In AdS space form factors are computed from the overlap integral of normalizable modes with boundary currents which propagate in AdS space. The AdS/CFT duality incorporates the connection between the twist-scaling dimension of the QCD boundary interpolating operators with the falloff of the normalizable modes in AdS near its conformal boundary [55]. If both quantities represent the same physical observable, a precise correspondence can be established at any momentum transfer between the string modes $\Phi$ in AdS space and the light front wave functions of hadrons $\psi$ in physical four-dimensional space-time [75]. In fact, light-front holographic methods were originally derived by observing the correspondence between matrix elements obtained in AdS/CFT with the corresponding formula using the light-front representation [75]. As shown in Chapter 4 the same results follow from comparing the relativistic light-front Hamiltonian equation describing bound states in $\mathrm{QCD}$ with the wave equations describing the 
propagation of modes in a warped AdS space for arbitrary spin [74, 91].

Form factors are among the most basic observables of hadrons, and thus central for our understanding of hadronic structure and dynamics. The physics includes the important interplay of perturbative and nonperturbative elements, which if properly taken into account, should allow us to study the transition from perturbative dynamics at large momentum transfer $q^{2}$ to non-perturbative dynamics at moderate and small $q^{2}$. Thus, the transition from quark and gluon degrees of freedom to hadronic degrees of freedom, which is not a simple task.

As will become clear from our discussion in this Chapter, holographic QCD incorporates important elements for the study of hadronic form factors which encompasses perturbative and nonperturbative elements, such as the connection between the twist of the hadron to the fall-off of its current matrix elements for large $q^{2}$, and essential aspects of vector meson dominance which are relevant at lower energies. This framework is also useful for analytically continuing the space-like results to the time-like region using simple analytic formulas expressed in terms of vector meson masses.

\subsection{Meson electromagnetic form factor}

\subsubsection{Meson form factor in AdS space}

In the higher dimensional gravity theory, the hadronic transition matrix element corresponds to the coupling of an external electromagnetic (EM) field $A^{M}(x, z)$, for a photon propagating in AdS space, with the extended field $\Phi_{P}(x, z)$ describing a hadron in AdS [77]. To simplify the discussion we treat here first the electromagnetic form factor for a spinless particle in a model with a wall at a finite distance $z=1 / \Lambda_{\mathrm{QCD}}$ - the hard wall model, which limits the propagation of the string modes in AdS space beyond the IR boundary, and also sets the hadronic mass scale [55]. The coupling of the EM field $A^{M}(x, z)$ follows from minimal coupling by replacing in (4.14) or (4.16) the covariant derivative $D_{M}$ by $D_{M}-i \mathrm{e}_{5} A_{M}$, where $\mathrm{e}_{5}$ is the charge in the bulk theory. To first order in the EM field the interaction term is

$$
S_{i n t}=\mathrm{e}_{5} \int d^{4} x d z \sqrt{g} g^{M M^{\prime}} \Phi^{*}(x, z) i{\overleftrightarrow{\partial_{M}}} \Phi(x, z) A_{M^{\prime}}(x, z)
$$

where $g \equiv\left|\operatorname{det} g_{\mathrm{MN}}\right|$. We recall from Chapter 4 that the coordinates of $\mathrm{AdS}_{5}$ are the Minkowski coordinates $x^{\mu}$ and $z$ labeled $x^{M}=\left(x^{\mu}, z\right)$, with $M, N=0, \cdots 4$, and $g$ is the 
determinant of the metric tensor. The hadronic transition matrix element has thus the form $[77]$

$$
\mathrm{e}_{5} \int d^{4} x d z \sqrt{g} \Phi_{P^{\prime}}^{*}(x, z) \overleftrightarrow{\partial}_{M} \Phi_{P}(x, z) A^{M}(x, z) \sim(2 \pi)^{4} \delta^{4}\left(P^{\prime}-P-q\right) \epsilon_{\mu}\left(P+P^{\prime}\right)^{\mu} \mathrm{e} F\left(q^{2}\right)
$$

where, the pion has initial and final four momentum $P$ and $P^{\prime}$ respectively and $q$ is the four-momentum transferred to the pion by the photon with polarization $\epsilon_{\mu}$. The expression on the right-hand side of (6.2) represents the space-like QCD electromagnetic transition amplitude in physical space-time

$$
\left\langle P^{\prime}\left|J^{\mu}(0)\right| P\right\rangle=\left(P+P^{\prime}\right)^{\mu} F\left(q^{2}\right)
$$

It is the EM matrix element of the quark current $J^{\mu}=e_{q} \bar{q} \gamma^{\mu} q$, and represents a local coupling to pointlike constituents. Although the expressions for the transition amplitudes look very different, one can show that a precise mapping of the matrix elements can be carried out at fixed light-front time for an arbitrary number of partons in the bound-state $[75,76]$.

The propagation of the pion in AdS space is described by a normalizable mode $\Phi_{P}\left(x^{\mu}, z\right)=e^{i P \cdot x} \Phi(z)$ with invariant mass $P_{\mu} P^{\mu}=M^{2}$ and plane waves along the physical coordinates $x^{\mu}$. The physical incoming electromagnetic probe (no physical polarizations along the AdS coordinate $z$ ) propagates in AdS according to

$$
A_{\mu}\left(x^{\mu}, z\right)=e^{i q \cdot x} V\left(q^{2}, z\right) \epsilon_{\mu}(q), \quad A_{z}=0
$$

where $\epsilon$ is the EM polarization vector in 4 dimensions, with $q \cdot \epsilon=0$. The function $V\left(q^{2}, z\right)$ - the bulk-to-boundary propagator, has the value 1 at zero momentum transfer, since we are normalizing the solutions to the total charge operator. It also has the value 1 at $z=0$, since the boundary limit is the external current: $A_{\mu}\left(x^{\mu}, z \rightarrow 0\right)=e^{i q \cdot x} \epsilon_{\mu}(q)$. Thus

$$
V\left(q^{2}=0, z\right)=V\left(q^{2}, z=0\right)=1 .
$$

Extracting the overall factor $(2 \pi)^{4} \delta^{4}\left(P^{\prime}-P-q\right)$ from momentum conservation at the vertex, which arises from integration over Minkowski variables in (6.2), we find [77]

$$
F\left(q^{2}\right)=R^{3} \int_{0}^{1 / \Lambda_{\mathrm{QCD}}} \frac{d z}{z^{3}} V\left(q^{2}, z\right) \Phi^{2}(z)
$$


where $F(0)=1$. The pion form factor in AdS is the overlap of the normalizable modes corresponding to the incoming and outgoing hadrons $\Phi_{P}$ and $\Phi_{P^{\prime}}$ with the nonnormalizable mode $V\left(q^{2}, z\right)$, corresponding to the external EM current [77] ${ }^{1}$.

\subsubsection{Meson form factor in light-front QCD}

The light-front formalism provides an exact Lorentz-invariant representation of current matrix elements in terms of the overlap of light-front wave functions. The electromagnetic current has elementary couplings to the charged constituents since the full Heisenberg current can be replaced in the interaction picture by the free quark current $J^{\mu}(0)$, evaluated at fixed light-cone time $x^{+}=0$ in the $q^{+}=0$ frame [78]. In contrast to the covariant Bethe-Salpeter equation, in the light front Fock expansion one does not need to include the contributions to the current from an infinite number of irreducible kernels, or the interactions of the electromagnetic current with vacuum fluctuations [73].

In the front-form, the EM form factor is most conveniently computed from the matrix elements of the plus component of the current component $J^{+}$at LF time $x^{+}=0$

$$
\left\langle P^{\prime}\left|J^{+}(0)\right| P\right\rangle=\left(P+P^{\prime}\right)^{+} F\left(q^{2}\right)
$$

This component of the current does not couple to Fock states with different numbers of constituents in the $q^{+}=0$ frame [78]. We express the plus component of the current operator

$$
J^{+}(x)=\sum_{q} e_{q} \bar{\psi}(x) \gamma^{+} \psi(x)
$$

in the particle number representation from the momentum expansion of $\psi(x)$ in terms of creation and annihilation operators $(2.14)^{2}$. The matrix element (6.7) is then computed by expanding the initial and final meson states $\left|\psi_{M}\left(P^{+}, \mathbf{P}_{\perp}\right)\right\rangle$ in terms of its Fock components (2.23). Using the normalization condition (2.22) for each individual constituent, and after integration over the intermediate variables in the $q^{+}=0$ frame we obtain the Drell-Yan-West expression [78, 79]

$$
F_{M}\left(q^{2}\right)=\sum_{n} \int\left[d x_{i}\right]\left[d^{2} \mathbf{k}_{\perp i}\right] \sum_{j} e_{j} \psi_{n / M}^{*}\left(x_{i}, \mathbf{k}_{\perp i}^{\prime}, \lambda_{i}\right) \psi_{n / M}\left(x_{i}, \mathbf{k}_{\perp i}, \lambda_{i}\right)
$$

\footnotetext{
${ }^{1}$ The equivalent expression for a spin- $J$ meson is $F\left(q^{2}\right)=R^{3-2 J} \int \frac{d z}{z^{3-2 J}} V\left(q^{2}, z\right) \Phi_{J}^{2}(z)$, where the hadronic mode $\Phi_{J}$ is normalized according to (4.24) for $d=4$.

${ }^{2}$ Notice that $\gamma^{+}$conserves the spin component of the struck quark (Appendix B), and thus the current $J^{+}$only couples Fock states with the same number of constituents.
} 
where the phase space momentum integration $\left[d x_{i}\right]\left[d^{2} \mathbf{k}_{\perp i}\right]$ is given by (2.24) and (2.25), and the variables of the light-front Fock components in the final state are given by $\mathbf{k}_{\perp i}^{\prime}=\mathbf{k}_{\perp i}+\left(1-x_{i}\right) \mathbf{q}_{\perp}$ for a struck constituent quark and $\mathbf{k}_{\perp i}^{\prime}=\mathbf{k}_{\perp i}-x_{i} \mathbf{q}_{\perp}$ for each spectator. The formula is exact if the sum is over all Fock states $n$.

The form factor can also be conveniently written in impact space as a sum of overlap of LFWFs of the $j=1,2, \cdots, n-1$ spectator constituents [167]. Suppose that the charged parton $n$ is the active constituent struck by the current, and the quarks $i=1,2, \cdots, n-1$ are spectators. We substitute (2.35) in the DYW formula (6.9). Integration over $k_{\perp}$ phase space gives us $n-1$ delta functions to integrate over the $n-1$ intermediate transverse variables with the result

$$
F_{M}\left(q^{2}\right)=\sum_{n} \prod_{j=1}^{n-1} \int d x_{j} d^{2} \mathbf{b}_{\perp j} \exp \left(i \mathbf{q}_{\perp} \cdot \sum_{j=1}^{n-1} x_{j} \mathbf{b}_{\perp j}\right)\left|\psi_{n / M}\left(x_{j}, \mathbf{b}_{\perp j}\right)\right|^{2}
$$

corresponding to a change of transverse momentum $x_{j} \mathbf{q}_{\perp}$ for each of the $n-1$ spectators. This is a convenient form for comparison with AdS results, since the form factor is expressed in terms of the product of light-front wave functions with identical variables.

\subsubsection{Light-front holographic mapping}

We now have all the elements to establish a connection of the AdS and light-front formulas. For definiteness we shall consider the $\pi^{+}$valence Fock state $|u \bar{d}\rangle$ with charges

$e_{u}=\frac{2}{3}$ and $e_{\bar{d}}=\frac{1}{3}$. For $n=2$, there are two terms which contribute to Eq. (6.10). Integrating over angles and exchanging $x \leftrightarrow 1-x$ in the second integral we find

$$
F_{\pi^{+}}\left(q^{2}\right)=2 \pi \int_{0}^{1} \frac{d x}{x(1-x)} \int \zeta d \zeta J_{0}\left(\zeta q \sqrt{\frac{1-x}{x}}\right)\left|\psi_{u \bar{d} / \pi}(x, \zeta)\right|^{2}
$$

where $\zeta^{2}=x(1-x) \mathbf{b}_{\perp}^{2}$ and $F_{\pi^{+}}(0)=1$.

We now compare this result with the electromagnetic form factor in AdS. Conserved currents are not renormalized and correspond to five-dimensional massless fields propagating in $\mathrm{AdS}_{5}$ space according to the relation $(\mu R)^{2}=(\Delta-p)(\Delta-4+p)$ for a $p$-form field in AdS space (I.22). This corresponds for $\mu=0$ and $p=1$ to either $\Delta=3$ or 1 , the canonical dimensions of an EM current and the massless gauge field respectively. The equation of motion describing the propagation of the electromagnetic field in AdS space 
is obtained from the action

$$
S_{e m}=\int d^{d} x d z \sqrt{g} g^{M M^{\prime}} g^{N N^{\prime}} F_{M N} F_{M^{\prime} N^{\prime}}
$$

with the covariant field tensor $F_{M N}=\partial_{M} A_{N}-\partial_{N} A_{M}$. It gives for $V\left(Q^{2}, z\right)($ Eq. (6.4)) the wave equation

$$
\left[\frac{d^{2}}{d z^{2}}-\frac{1}{z} \frac{d}{d z}-Q^{2}\right] V\left(Q^{2}, z\right)=0
$$

where $Q^{2}=-q^{2}>0$. Its solution, subject to the boundary conditions $(6.5)$, is

$$
V\left(Q^{2}, z\right)=z Q K_{1}(z Q)
$$

which decays exponentially for large values of $Q^{2}$ : here $K_{1}(Q z) \sim \sqrt{\frac{\pi}{2 Q z}} e^{-Q z 3}$. Using the integral representation of $V\left(Q^{2}, z\right)$ from (G.17)

$$
V\left(Q^{2}, z\right)=\int_{0}^{1} d x J_{0}\left(z Q \sqrt{\frac{1-x}{x}}\right)
$$

we can write the AdS electromagnetic form-factor as

$$
F\left(Q^{2}\right)=R^{3} \int_{0}^{1} d x \int \frac{d z}{z^{3}} J_{0}\left(z Q \sqrt{\frac{1-x}{x}}\right) \Phi^{2}(z) .
$$

To compare with the light-front QCD form factor expression (6.11) we use the expression of the light-front wave function (2.40)

$$
\psi(x, \zeta, \varphi)=e^{i L \varphi} X(x) \frac{\phi(\zeta)}{\sqrt{2 \pi \zeta}}
$$

which we use to factor out the longitudinal and transverse modes $\phi(\zeta)$ and $X(x)$ in (6.11). Both expressions for the form factor have to be identical for arbitrary values of $Q$. We obtain the result $[75]$

$$
\phi(\zeta)=\left(\frac{R}{\zeta}\right)^{-3 / 2} \Phi(\zeta) \quad \text { and } \quad X(x)=\sqrt{x(1-x)}
$$

\footnotetext{
${ }^{3}$ This solution corresponds to a "free" EM current in physical space. Confined EM currents in AdS correspond to "dressed" currents in QCD. This will be discussed in the next section.
} 
where we identify the transverse impact LF variable $\zeta$ with the holographic variable $z, z \rightarrow \zeta=\sqrt{x(1-x)}\left|\mathbf{b}_{\perp}\right| .{ }^{4}$ Thus, in addition of recovering the expression found in Chapter 5, which relates the transverse mode $\phi(\zeta)$ in physical space-time to the field $\Phi(z)$ in AdS space from the mapping to the LF Hamiltonian equations, we find a definite expression for the longitudinal LF mode $X(x)^{5}$. The identical result follows from mapping the matrix elements of the energy-momentum tensor [81] (See Appendix E).

Although the expression for the form-factor (6.6) is derived in the simple hard-wall model, the power falloff for large $Q^{2}$ is model independent. This follows from the fact that the leading large- $Q^{2}$ behavior of form factors in AdS/QCD arises from the small $z \sim 1 / Q$ kinematic domain in AdS space. According to the AdS/CFT duality (See Chapter 1), this corresponds to small distances $x_{\mu} x^{\mu} \sim 1 / Q^{2}$ in physical space-time, the domain where the current matrix elements are controlled by the conformal twistdimension $\tau$ of the hadron's interpolating operator. In the case of the front form, where $x^{+}=0$, this corresponds to small transverse separation $x_{\mu} x^{\mu}=-\mathbf{x}_{\perp}^{2}$. In general, the short-distance behavior of a hadronic state is characterized by its twist (dimension minus spin) $\tau=\Delta-\sigma$, where $\sigma$ is the sum over the constituent's spin $\sigma=\sum_{i=1}^{n} \sigma_{i}$. Twist is also equal to the number of partons $\tau=n^{6}$.

In a high-energy electron-proton elastic collision experiment, for example, the photon propagation is near to the light-cone, and thus its short space-like interval maps to the boundary of AdS near $z=0$ (Chapter 1). This means that the photon propagation function $V\left(Q^{2}, z\right)$ is strongly suppressed in the AdS interior. At large enough $Q^{2}$ the important contribution to the integral in (6.6) is from the asymptotic boundary region near $z \sim 1 / Q$ where the function $V\left(Q^{2}, z\right)$ has its important support. At small $z$ the string modes scale as $\Phi \sim z^{\Delta}$, and the ultraviolet point-like power-scaling behavior (instead of a soft collision amplitude) is recovered [55]

$$
F\left(Q^{2}\right) \rightarrow\left[\frac{\Lambda_{\mathrm{QCD}}^{2}}{Q^{2}}\right]^{\Delta-1}
$$

\footnotetext{
${ }^{4}$ Extension of the results to arbitrary $n$ follows from the $x$-weighted definition of the transverse impact variable of the $n-1$ spectator system given by Eq. (2.47). In general the mapping relates the AdS density $\Phi^{2}(z)$ to an effective LF single particle transverse density [75].

${ }^{5}$ It is interesting to notice that computations based on lattice QCD and rainbow-ladder truncation of Dyson-Schwinger equations of twist-two parton distribution amplitudes give similar results for the longitudinal component $X(x)[267,268]$.

${ }^{6}$ For a hadronic state with relative orbital angular momentum $L$ the twist is $\tau=n+L$.
} 
upon the substitution $\Delta \rightarrow n$.

It is remarkable that the QCD dimensional counting rules $[56,57,58]$ are also a key feature of nonperturbative models [55] based on the gauge/gravity duality. If fact, the phenomenological success of dimensional scaling laws implies that QCD is a strongly coupled conformal theory at moderate, but not asymptotic, energies ${ }^{7}$. In hard exclusive scattering there is little sign of the logarithmic running of the QCD coupling from QCD perturbative predictions [159]. For example, the measured proton Dirac form factor $F_{1}$ scales as $Q^{4} F_{1}\left(Q^{2}\right) \simeq$ constant, up to $Q^{2} \leq 35 \mathrm{GeV}^{2}$ [269]. This puzzling behavior could have an explanation in the fact that in exclusive reactions the virtualty of the gluons exchanged in the hard QCD processes is typically much less than the momentum transfer scale $Q$, since several gluons share the total momentum transfer, and thus the $Q^{2}$-independence of the strong coupling is tested in the conformal IR window. Since the simple nonperturbative counting rules (6.19) encode the conformal aspects of the theory, the holographic predictions seem to explain quite well the exclusive data in this large, but not asymptotically large energy range.

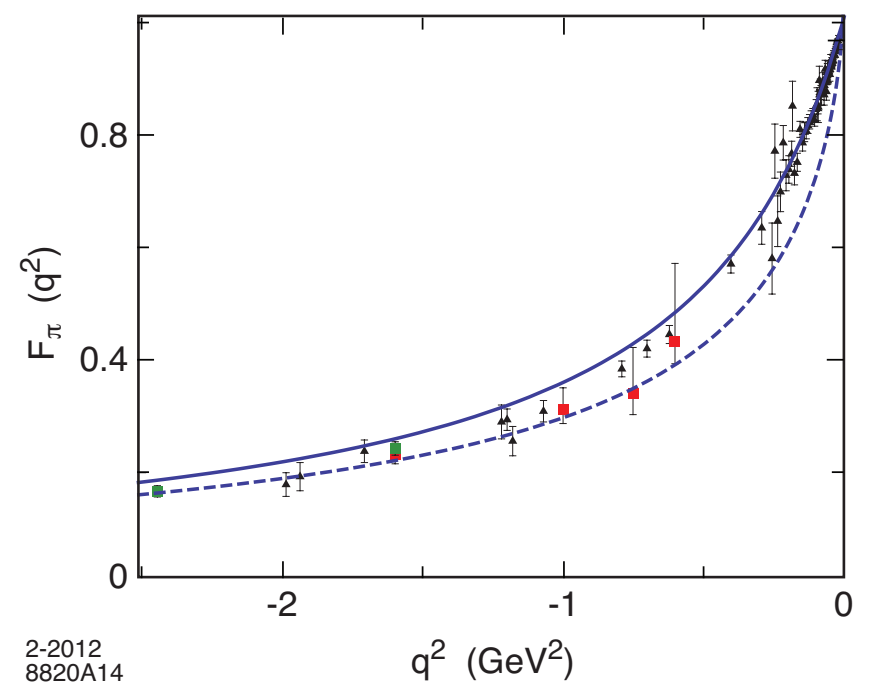

Figure 6.1: Space-like electromagnetic pion form factor $F_{\pi}\left(q^{2}\right)$. Continuous line: confined current, dashed line: free current. Triangles are the data compilation from Baldini [271], boxes are JLAB data [272, 273].

The results for the elastic form factor described above correspond to a 'free' current propagating on AdS space. It is dual to the electromagnetic point-like current in the Drell-Yan-West light-front formula $[78,79]$ for the pion form factor. The DYW formula

\footnotetext{
${ }^{7}$ For small $z$ it follows that $\phi_{J} \sim z^{1 / 2+L}$ and thus from $(5.1) \Phi_{J} \sim z^{3 / 2-J} \phi_{J} \sim z^{2+L-J}$, in agreement with the pion twist $\tau=n+L$ for $n=2$.
} 
is an exact expression for the form factor. It is written as an infinite sum of an overlap of LF Fock components with an arbitrary number of constituents. This allows one to map state-by-state to the effective gravity theory in AdS space. However, this mapping has the shortcoming that the nonperturbative pole structure of the time-like form factor does not appear in the time-like region unless an infinite number of Fock states is included. Furthermore, the moments of the form factor at $Q^{2}=0$ diverge term-by-term; for example one obtains an infinite charge radius [270] as shown in Fig. $6.1^{8}$ In fact, infinite slopes also occur in chiral theories when coupling to a massless pion.

\section{Pion form factor with confined AdS current}

The description of form factors in AdS has the feature that the time-like pole structure is incorporated in the EM current when the current is 'confined', $i$. e., the EM current is modified as it propagates in an IR modified AdS space to incorporate confinement. In this case, the confined current in AdS is dual to a hadronic EM current which includes any number of virtual $q \bar{q}$ components. The confined EM current also leads to finite moments at $Q^{2}=0$, since a hadronic scale is incorporated in the EM current. This is illustrated in Fig. 6.1 for the EM pion form factor.

As a specific example, consider the hard-wall model with a wall at a finite distance $z=1 / \Lambda_{\mathrm{QCD}}{ }^{9}$. The gauge-invariant boundary conditions for the confined EM field lead to the expression [274]

$$
V\left(Q^{2}, z\right)=z Q\left[K_{1}(z Q)+I_{1}(z Q) \frac{K_{0}\left(Q / \Lambda_{\mathrm{QCD}}\right)}{I_{0}\left(Q / \Lambda_{\mathrm{QCD}}\right)}\right],
$$

for the bulk-to-boundary propagator, where an infinite series of time-like poles in the confined AdS current corresponds to the zeros of the Bessel function $I_{0}\left(Q / \Lambda_{\mathrm{QCD}}\right)$. This is conceptually very satisfying, since by using the relation $J_{\alpha}(i x)=e^{i \alpha \pi / 2} I_{\alpha}(x)$ for the modified Bessel function $I_{\alpha}(x)$, it follows that the poles in (6.20) are determined by the dimension 2 solution of the hadronic wave equation for $L=0$, even if the EM current itself scales with dimension 3. Thus, the poles in the current correspond to the mass spectrum of radial excitations computed in the hard-wall model. Notice that the scaling dimension 2 corresponds to leading twist $\tau=2$ quark-antiquark bound state,

\footnotetext{
${ }^{8}$ This deficiency is solved by taking into account finite quark masses. In this case the charge radius becomes finite for free currents in the DYW formula.

${ }^{9} \mathrm{~A}$ logarithmically divergent result for the pion radius does not appear in the hard-wall model if one uses Neumann boundary conditions for the EM current. In this case the EM current is confined and $\left\langle r_{\pi}^{2}\right\rangle \sim 1 / \Lambda_{\mathrm{QCD}}^{2}$
} 
whereas the scaling dimension 3 corresponds to the naive conformal dimension of the EM conserved current. The downside of the hard-wall model, however, is that the spectrum of radial excitations in this model behaves as $M \sim 2 n$, and thus this model is not able to describe correctly the radial vector-meson excitations, and consequently neither the time-like form factor data. The observed $\rho$ vector-meson radial trajectory has instead the Regge behavior $M^{2} \sim n$. In the limit for large $Q^{2}$ we recover the 'free' current propagating in AdS, given by (6.14). A discussion of pion and vector form factors in the hard-wall model is given in Refs. [274, 275, 276, 277, 278, 279].

\subsubsection{Soft-wall form factor model}

We can extend the computation of form factors with a confined current for the soft wall model. For a general dilaton profile one need to introduce a $z$-dependent AdS effective coupling $e_{5}(z)$. This procedure does not affect gauge symmetry in asymptotic physical Minkowski space, and generally for any fixed value of the holographic variable $z$. As it turns out, the functional dependence on $z$ is determined by the requirements of charge conservation in Minkowski space at $Q^{2}=0$. This is analogous to the introduction of a $z$-dependent mass in (4.16), which was fixed by the requirement of a separation of dynamical and kinematical features. Following the same steps as for the hard-wall model discussed above, we find for the pion form factor

$$
\mathrm{e} F\left(Q^{2}\right)=R^{3} \int \frac{d z}{z^{3}} e^{\varphi(z)} \mathrm{e}_{5}(z) V\left(Q^{2}, z\right) \Phi^{2}(z)
$$

with boundary conditions

$$
\frac{1}{\mathrm{e}} \lim _{Q^{2} \rightarrow 0} e_{5}(z) V\left(Q^{2}, z\right)=\frac{1}{\mathrm{e}} \lim _{z \rightarrow 0} e_{5}(z) V\left(Q^{2}, z\right)=1
$$

To find the behavior of the bulk-to-boundary propagator we consider the dilatonmodified action for the EM field in AdS

$$
S_{e m}=\int d^{d} x d z \sqrt{g} e^{\varphi(z)} g^{M M^{\prime}} g^{N N^{\prime}} F_{M N} F_{M^{\prime} N^{\prime}}
$$

Its variation gives the wave equation

$$
\left[\frac{d^{2}}{d z^{2}}-\left(\frac{1}{z}-\varphi^{\prime}(z)\right) \frac{d}{d z}-Q^{2}\right] V\left(Q^{2}, z\right)=0
$$


For the harmonic dilaton profile $\varphi(z)=\lambda z^{2}$ its non-normalizable solution is the EM bulk-to-boundary propagator [76, 280] (See Appendix G.1)

$$
V\left(Q^{2}, z\right)=e^{(-|\lambda|-\lambda) z^{2} / 2} \Gamma\left[1+\frac{Q^{2}}{4|\lambda|}\right] U\left[\frac{Q^{2}}{4|\lambda|}, 0,|\lambda| z^{2}\right]
$$

where $U(a, b, c)$ is the Tricomi confluent hypergeometric function

$$
\Gamma(a) U(a, b, z)=\int_{0}^{\infty} e^{-z t} t^{a-1}(1+t)^{b-a-1} d t .
$$

The current (6.25) has the limiting values $V(0, z)=e^{(-|\lambda|-\lambda) z^{2} / 2}$ and $V\left(Q^{2}, 0\right)=1$.

We can determine the $z$-dependence of the AdS coupling $\mathrm{e}_{5}$ from charge conservation, $F(0)=1$, in the limit $Q \rightarrow 0$ using (6.22). This requirements fixes for $\lambda<0$ the $z$ dependence of $e_{5}(z)=\mathrm{e}$ independent of $z$, and for $\lambda>0$ to $\mathrm{e}_{5}=\mathrm{e} e^{\lambda z^{2}}$. Thus the effective current is

$$
\tilde{V}\left(Q^{2}, z\right)=\Gamma\left[1+\frac{Q^{2}}{4|\lambda|}\right] U\left[\frac{Q^{2}}{4|\lambda|}, 0,|\lambda| z^{2}\right]
$$

where $\tilde{V}(z)=\frac{1}{\mathrm{e}} \mathrm{e}_{5} V(z)$. The modified current $\tilde{V}\left(Q^{2}, z\right)$, Eq. (6.27), has the same boundary conditions (6.5) as the free current (6.14), and reduces to (6.14) in the limit $Q^{2} \rightarrow \infty[76]$.

The soft-wall model of confinement [61] also has important analytical properties which are particularly useful for the study of transition amplitudes. As shown in Ref. [280] the bulk-to-boundary propagator (6.27) has the integral representation

$$
\tilde{V}\left(Q^{2}, z\right)=|\lambda| z^{2} \int_{0}^{1} \frac{d x}{(1-x)^{2}} x^{Q^{2} / 4|\lambda|} e^{-|\lambda| z^{2} x /(1-x)} .
$$

Since the integrand in (6.28) contains the generating function of the associated Laguerre polynomials

$$
\frac{e^{-|\lambda| z^{2} x /(1-x)}}{(1-x)^{k+1}}=\sum_{n=0}^{\infty} L_{n}^{k}\left(|\lambda| z^{2}\right) x^{n}
$$

$\tilde{V}\left(Q^{2}, z\right)$ can thus be expressed as a sum of poles [280]

$$
\tilde{V}\left(Q^{2}, z\right)=4 \lambda^{2} z^{2} \sum_{n=0}^{\infty} \frac{L_{n}^{1}\left(|\lambda| z^{2}\right)}{M_{n}^{2}+Q^{2}}
$$


with $M_{n}^{2}=4|\lambda|(n+1)$.

For negative values of $Q^{2}$ (time-like), the poles of the dressed current (6.30) occur at $-Q^{2}=4|\lambda|(n+1)$. On the other hand, the poles of the observed vector mesons with quantum numbers $J=1, L=0$ according to the bound-state equation (5.8), should occur at $-Q^{2}=4|\lambda|\left(n+\frac{1}{2}\right)^{10}$. From here on, we will shift the vector mesons mass poles to their physical twist-2 location to obtain a meaningful comparison with measurements. When this is done, the agreement with data is very good [176].

Let us now compute the elastic EM form factor corresponding to the lowest radial $n=0$ and orbital $L=0$ state for an arbitrary twist $\tau$ described by the hadronic state

$$
\Phi_{\tau}(z)=\sqrt{\frac{2}{\Gamma(\tau-1)}} \kappa^{\tau-1} z^{\tau} e^{-\kappa^{2} z^{2} / 2}
$$

with normalization.

$$
\left\langle\Phi_{\tau} \mid \Phi_{\tau}\right\rangle=\int \frac{d z}{z^{3}} \Phi_{\tau}(z)^{2}=1 .
$$

This agrees with the fact that the field $\Phi_{\tau}$ couples to a local hadronic interpolating operator of twist $\tau$ defined at the asymptotic boundary of AdS space, and thus the scaling dimension of $\Phi_{\tau}$ is $\tau$. For convenience we have redefined the wave function to absorb the dilaton profile. To compute the form factor

$$
F_{\tau}\left(Q^{2}\right)=R^{3} \int \frac{d z}{z^{3}} \tilde{V}\left(Q^{2}, z\right) \Phi_{\tau}^{2}(z)
$$

we substitute in (6.33) the field (6.31) and the bulk-to-boundary propagator (6.28). Upon integration over the variable $z$ we find the result [76]

$$
F_{\tau}\left(Q^{2}\right)=\int_{0}^{1} d x \rho_{\tau}(x, Q)
$$

where

$$
\rho_{\tau}(x, Q)=(\tau-1)(1-x)^{\tau-2} x^{\frac{Q^{2}}{4 \kappa^{2}}}
$$

\footnotetext{
${ }^{10}$ For a negative dilaton profile $[61]$ the vector meson radial trajectory corresponds to the quantum numbers $J=L=1$ and thus the poles are located at $-Q^{2}=4|\lambda|(n+1)$. This identification, however, is not compatible with light-front QCD.
} 
The integral (6.34) can be expressed in terms of Gamma functions

$$
F_{\tau}\left(Q^{2}\right)=\Gamma(\tau) \frac{\Gamma\left(1+\frac{Q^{2}}{4 \kappa^{2}}\right)}{\Gamma\left(\tau+\frac{Q^{2}}{4 \kappa^{2}}\right)} .
$$

For integer twist- $\tau$ (the number of constituents $N$ ) for a given Fock component we find $[76]$

$$
F_{\tau}\left(Q^{2}\right)=\frac{1}{\left(1+\frac{Q^{2}}{\mathcal{M}_{\rho}^{2}}\right)\left(1+\frac{Q^{2}}{\mathcal{M}_{\rho^{\prime}}^{2}}\right) \cdots\left(1+\frac{Q^{2}}{\mathcal{M}_{\rho^{\tau-2}}^{2}}\right)},
$$

which is expressed as a $\tau-1$ product of poles along the vector meson Regge radial trajectory. For a pion, for example, its lowest Fock state - the valence state - is a twist-2 state, and thus the form factor is the well known monopole form [76]. For the proton, the minimal Fock state is a twist-3 state, and the corresponding form factor is the product of two monopoles, corresponding to the two lowest vector meson states. It is important to notice that even if the confined EM dressed current (6.30) contains an infinite number of poles, the actual number of poles appearing in the expression for the elastic form factor (6.37) is determined by the twist of the Fock component: the resulting form factor is given by a product of $N-1$ poles for an $N$-component Fock state. The remarkable analytical form of (6.37), expressed in terms of the lowest vector meson mass and its radial excitations, incorporates not only the correct leading-twist scaling behavior expected from the constituent's hard scattering with the photon but also vector meson dominance (VMD) al low energy [281] and a finite mean-square charge radius $\left\langle r^{2}\right\rangle \sim \frac{1}{\lambda}^{11}$. The light-front holographic approach extends the traditional Sakurai form of vector meson dominance [281] to a product of vector poles. Since the LF holographic amplitude encodes the power-law behavior for hard-scattering [56, 57, 58], the result (6.37) can be extended naturally to high-energies, thus overcoming the limitations of the original VMD model [282] ${ }^{12}$.

\section{Effective wave function from holographic mapping of a current}

It is also possible to find a precise mapping of a confined EM current propagating in a warped AdS space to the light-front QCD Drell-Yan-West expression for the form

\footnotetext{
${ }^{11}$ In contrast, the computation with a free current gives the logarithmically divergent result $\left\langle r^{2}\right\rangle \sim$ $\left.\frac{1}{\lambda} \ln \left(\frac{4 \kappa^{2}}{Q^{2}}\right)\right|_{Q^{2} \rightarrow 0}$.

${ }^{12}$ Other extensions are discussed for example in Ref. [283].
} 
factor. In this case we find an effective LFWF, which corresponds to a superposition of an infinite number of Fock states generated by the "dressed" confined current. For the soft-wall model this mapping can be done analytically.

The form factor in light-front QCD can be expressed in terms of an effective singleparticle density [167]

$$
F\left(Q^{2}\right)=\int_{0}^{1} d x \rho(x, Q),
$$

where $\rho(x, Q)=2 \pi \int_{0}^{\infty} b d b J_{0}(b Q(1-x))|\psi(x, b)|^{2}$, for a two-parton state $\left(b=\left|\mathbf{b}_{\perp}\right|\right)$. By direct comparison with (6.35) for arbitrary values of $Q^{2}$ we find the effective two-parton LFWF [284]

$$
\psi_{\text {eff }}\left(x, \mathbf{b}_{\perp}\right)=\kappa \frac{(1-x)}{\sqrt{\pi \ln \left(\frac{1}{x}\right)}} e^{-\frac{1}{2} \kappa^{2} \mathbf{b}_{\perp}^{2}(1-x)^{2} / \ln \left(\frac{1}{x}\right)},
$$

in impact space. The momentum space expression follows from the Fourier transform of (6.39) and it is given by [284]

$$
\psi_{\text {eff }}\left(x, \mathbf{k}_{\perp}\right)=4 \pi \frac{\sqrt{\ln \left(\frac{1}{x}\right)}}{\kappa(1-x)} e^{-\mathbf{k}_{\perp}^{2} / 2 \kappa^{2}(1-x)^{2} \ln \left(\frac{1}{x}\right)} .
$$

The effective LFWF encodes nonperturbative dynamical aspects that cannot be determined from a term-by-term holographic mapping, unless one includes an infinite number of terms. However, it has the correct analytical properties to reproduce the bound state vector meson pole in the pion time-like EM form factor. Unlike the "true" valence LFWF, the effective LFWF, which represents a sum of an infinite number of Fock components in the EM current, is not symmetric in the longitudinal variables $x$ and $1-x$ for the active and spectator quarks, respectively.

\section{Higher Fock components and form factors}

One can extend the formalism in order to examine the contribution of higher-Fock states in the nonperturbative analytic structure of time-like hadronic form factors. In fact, as we have shown above for the soft-wall model, there is a precise non-trivial relation between the twist (number of components) of each Fock state in a hadron and the number of poles from the hadronized $q \bar{q}$ components in the electromagnetic current inside the hadron. In general, the pion state is a superposition of an infinite number of 
Fock components $|N\rangle,|\pi\rangle=\sum_{N} \psi_{N}|N\rangle$, and thus the full pion form factor is given by

$$
F_{\pi}\left(q^{2}\right)=\sum_{\tau} P_{\tau} F_{\tau}\left(q^{2}\right)
$$

since the charge is a diagonal operator. Normalization at $Q^{2}=0, F_{\pi}(0)=1$, implies that $\sum_{\tau} P_{\tau}=1$ if all possible states are included.

Conventionally the analysis of form factors is based on the generalized vector meson dominance model

$$
F_{\pi}\left(q^{2}\right)=\sum_{\lambda} C_{\lambda} \frac{M_{\lambda}^{2}}{M_{\lambda}^{2}-q^{2}},
$$

with a dominant contribution from the $\rho$ vector meson plus contributions from the higher resonances $\rho^{\prime}, \rho^{\prime \prime}, \rho^{\prime \prime \prime}, \ldots$, etc [285]. Comparison with (6.41) and (6.37) allow us to determine the coefficients $C_{\lambda}$ in terms of the of the probabilities $P_{\tau}$ for each Fock state and the vector meson masses $M_{n}^{2}$. However, no fine tuning of the coefficients $C_{\lambda}$ is necessary in the holographic LF framework, since the correct scaling is incorporated in the model.

\subsubsection{Time-like form factors in holographic QCD}

The computation of form factors in the time-like region is in general a complex task. For example, lattice results of Minkowski observables, such as time-like hadronic form factors cannot be obtained directly from numerical Euclidean-space lattice simulations, and Dyson-Schwinger computations are often specific to the space-like region. Typically models based on hadronic degrees of freedom involve sums over a large number of intermediate states and thus require a large number of hadronic parameters [285, 286, 287, 288, 289]. In contrast, as we shall show below for an specific example, the analytical extension of the holographic model to $s>4 m_{\pi}^{2}$ encodes most relevant dynamical aspects of the time-like form factor, including the relative phases between different vector-meson resonance contributions.

In the strongly coupled semiclassical gauge/gravity limit, hadrons have zero widths and are stable, as in the $N_{C} \rightarrow \infty$ limit of QCD ${ }^{13}$. In a realistic theory, the resonances have widths due to their mixing with the continuum, e.g., two pions. As a practical

\footnotetext{
${ }^{13}$ In Refs. $[76,284]$ the computation of the pion leptonic decay constant in LF holography is examined. A computation of decay constants in the framework of bottom-up AdS/QCD models will be given in Chapter 5. See also [290] for a recent computation of the decay constant of the pion and its excited states.
} 
approach, we modify (6.37) by introducing finite widths in the expression for $F_{\pi}(s)$ according to

$$
F_{\tau}(s)=\frac{M_{\rho}^{2} M_{\rho^{\prime}}^{2} \cdots M_{\rho^{\tau-2}}^{2}}{\left(M_{\rho}^{2}-s-i \sqrt{s} \Gamma_{\rho}(s)\right)\left(M_{\rho^{\prime}}^{2}-s-i \sqrt{s} \Gamma_{\rho^{\prime}}(s)\right) \cdots\left(M_{\rho^{\tau-2}}^{2}-s-i \sqrt{s} \Gamma_{\rho^{\tau-2}}(s)\right)}
$$

with normalization $F_{\tau}(0)=1$.

The effect of multiparticle states coupled to $\rho$-resonances is to introduce an $s$ dependent width $\Gamma(s)$. The modified width include the kinematical factors from the mixing of the vector mesons with the continuum - which occurs mainly to pion pairs, although higher multiparticle states also occur at large $s^{14}$.

\section{A simple holographic model}

In holographic QCD, the Fock states of hadrons can have any number of extra $q \bar{q}$ pairs created by the confining potential; however, there are no constituent dynamical gluons [51]. This result is consistent with the flux-tube interpretation of QCD [49] where soft gluons interact strongly to build a color confining potential for quarks. Gluonic degrees of freedom only arise at high virtuality and gluons with smaller virtuality are sublimated in terms of the effective confining potential. This unusual property of QCD may explain the dominance of quark interchange [292] over quark annihilation or gluon exchange contributions in large-angle elastic scattering [293]. In fact, empirical evidence confirming gluonic degrees of freedom at small virtualities or constituent gluons is lacking [203, 294].

In a complete treatment the unstable hadron eigenvalues and widths should emerge due to mixing with the continuum. This is a formidable dynamical problem; thus to illustrate the relevance of higher Fock states in the analytic structure of the pion form factor, we will consider a simple phenomenological model where the widths are constant and basically taken from the Particle Data Group and the probabilities are taken as adjustable parameters. We will consider a simplified model [295] where we only include the first two components in a Fock expansion of the LF pion wave function

$$
|\pi\rangle=\psi_{q \bar{q} / \pi}|q \bar{q}\rangle_{\tau=2}+\psi_{q \bar{q} q \bar{q}}|q \bar{q} q \bar{q}\rangle_{\tau=4}+\cdots
$$

and no constituent dynamical gluons [51]. The $J^{P C}=0^{-+}$twist-2 and twist-4 states are

\footnotetext{
${ }^{14}$ An alternative form given by Gounaris and Sakurai is often used [291].
} 
created by the interpolating operators $\mathcal{O}_{2}=\bar{q} \gamma^{+} \gamma_{5} q$ and $\mathcal{O}_{4}=\bar{q} \gamma^{+} \gamma_{5} q \bar{q} q$. Up to twist-4 the corresponding expression for the pion form factor is

$$
F_{\pi}\left(q^{2}\right)=(1-\gamma) F_{\tau=2}\left(q^{2}\right)+\gamma F_{\tau=4}\left(q^{2}\right),
$$

where we have labeled the twist- 4 probability $P_{q \bar{q} q \bar{q}}=\gamma$, the admixture of the $|q \bar{q} q \bar{q}\rangle$ state.

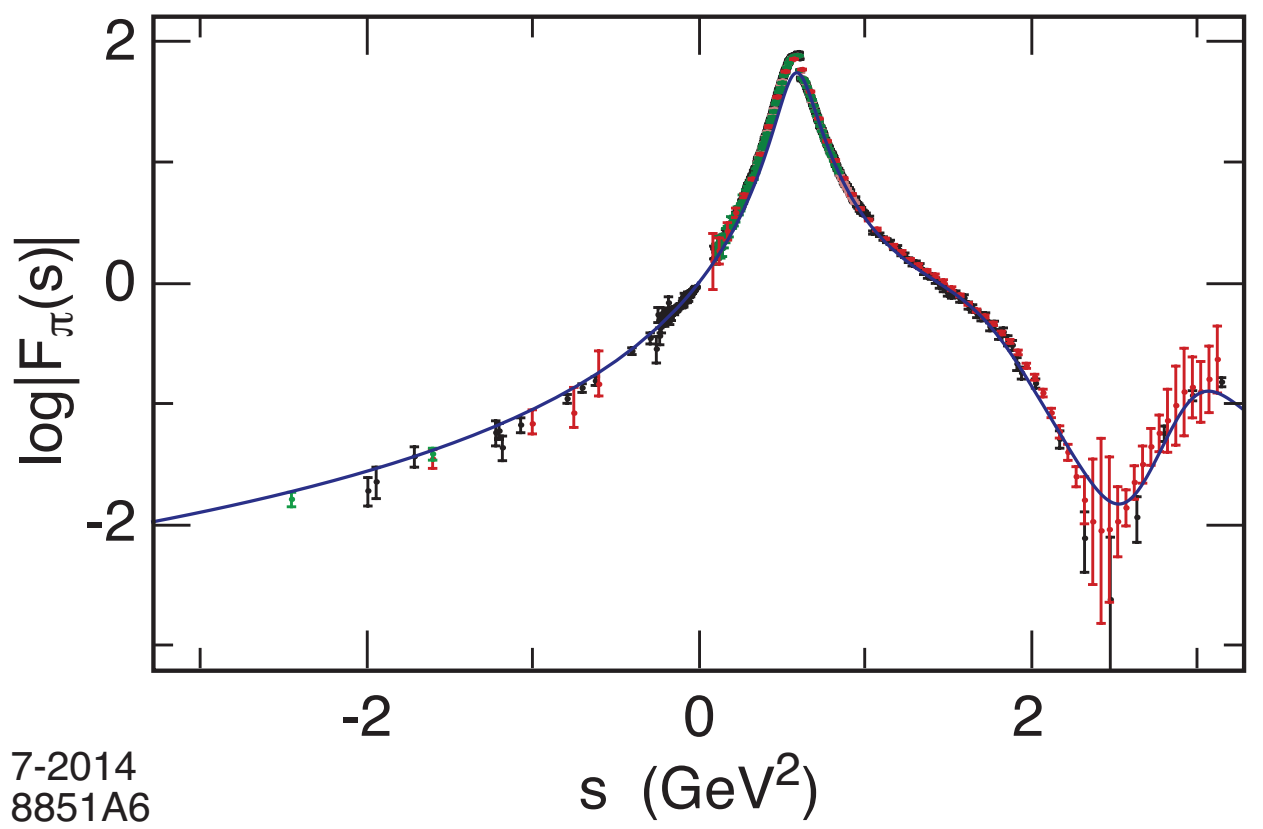

Figure 6.2: The structure of the space-like $\left(s=-Q^{2}<0\right)$ and time-like $\left(s=q^{2}>4 m_{\pi}^{2}\right)$ pion form factor in light-front holographic QCD for a truncation of the pion wave function up to twist four. The space-like data are taken from the compilation from Baldini et al. [271] (black) and JLAB data $[272,273]$ (red and green). The time-like data are from the precise measurements from KLOE [296, 297, 298] (dark green and dark red), BABAR [299, 300] (black) and BELLE [301] (red).

The predictions of the light-front holographic model up to twist-4 (6.45) for the space-like and time-like pion elastic form factor are shown in Fig. 6.2. We choose the values $\Gamma_{\rho}=149 \mathrm{MeV}, \Gamma_{\rho^{\prime}}=360 \mathrm{MeV}$ and $\Gamma_{\rho^{\prime \prime}}=160 \mathrm{MeV}$. The chosen values for the width of the $\rho^{\prime}$ and $\rho^{\prime \prime}$ are on the lower side of the PDG values listed in Ref. [16]. The results correspond to the probability $P_{q \bar{q} q \bar{q}}=12.5 \%$. The values of $P_{q \bar{q} q \bar{q}}$ (and the corresponding widths) are inputs in the model. We use the value of $\sqrt{\lambda}=0.5482 \mathrm{GeV}$ determined from the $\rho$ mass: $\sqrt{\lambda}=M_{\rho} / \sqrt{2}$, and the masses of the radial excitations follow from setting the poles at their physical locations, $M^{2} \rightarrow 4 \lambda(n+1 / 2)$. The 
main features of the pion form factor in the space-like and time-like regions are well described by the same physical picture with a minimal number of parameters. The value for the pion radius is $\left\langle r_{\pi}\right\rangle=0.644 \mathrm{fm}$, compared with the experimental value $\left\langle r_{\pi}\right\rangle=0.672 \pm 0.008 \mathrm{fm}$ from Ref. [16]. Since we are interested in the overall behavior of the model, we have not included $\omega-\rho$ mixing and kinematical threshold effects. This simple model, however, reproduces quite well the space- and time-like structure in the momentum transfer regime where the model is valid: this is, up to the second radial excitation of the $\rho, s \simeq M_{\rho^{\prime \prime}}^{2} \simeq 3 \mathrm{GeV}^{2}$ in the time like region (which covers the Belle data). Above this energy interference with higher twist contributions and a detailed study of the effects of the mixing of the vector mesons with the continuum should be incorporated, as well as the effects of $s$-dependent widths from multiparticle states coupled to the $\rho$-resonances.

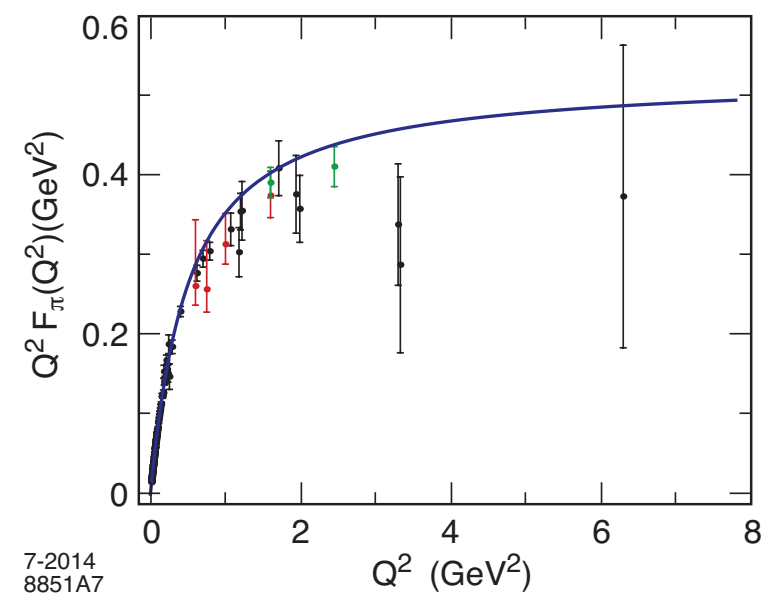

Figure 6.3: Scaling predictions for $Q^{2} F_{\pi}\left(Q^{2}\right)$. The space-like data is from the compilation of Baldini et al. (black) [271] and JLAB data [272, 273] (red and green).

The analytical structure of the holographic model encodes essential dynamical aspects of the pion form factor, including its pole and relative phase dependence as derived from multiple vector meson resonances, leading-twist scaling at high virtuality, as well as the transition from the hard-scattering domain to the long-range confining hadronic region. The scaling behavior in the space-like region is illustrated in Fig. 6.3 where we plot $Q^{2} F_{\pi}\left(Q^{2}\right)$.

At very large time-like momentum transfer we expect that higher twist contributions and the effects of the mixing with the continuum are not important. This follows from the structure of the hadronic form-factor $F_{\tau}(6.37)$, which is the product of $(N-1)$-poles for the twist $\tau=N$ component in the light-front Fock expansion of the wave function. As 
a result, the resonant contributions for twist $N+1$ is decoupled by a factor $1 / Q^{2}$ for large $Q^{2}$, compared to the twist $N$ contribution. Thus at large time-like momentum transfer, the resonant structure should be less and less visible and melts to a smooth curve. This is indeed the case for the Babar data which is well reproduced above $s \simeq 6 \mathrm{GeV}^{2}$ by the leading twist-2 amplitude. However, a recent measurement from CLEO [302] at $s=14.2$ and $17.4 \mathrm{GeV}^{2}$ gives results significantly higher than those expected from QCD scaling considerations. One can also extend the LF holographic approach to describe other processes, as for example the photon-to-meson transition form factors, such as $\gamma^{*} \gamma \rightarrow \pi^{0}$, a reaction which has been of intense experimental and theoretical interest ${ }^{15}$.

\subsection{Nucleon electromagnetic form factors}

Proton and neutron electromagnetic form factors are among the most basic observables of the nucleon, and thus central for our understanding of the nucleon's structure and dynamics ${ }^{16}$. In general two form factors are required to describe the elastic scattering of electrons by spin- $\frac{1}{2}$ nucleons, the Dirac and Pauli form factors, $F_{1}$ and $F_{2}$

$$
\left\langle P^{\prime}\left|J^{\mu}(0)\right| P\right\rangle=u\left(P^{\prime}\right)\left[\gamma^{\mu} F_{1}\left(q^{2}\right)+\frac{i \sigma^{\mu \nu} q^{\nu}}{2 \mathcal{M}} F_{2}\left(q^{2}\right)\right] u(P),
$$

where $q=P^{\prime}-P$. In the light-front formalism one can identify the Dirac and Pauli form factors from the LF spin-conserving and spin-flip current matrix elements of the $J^{+}$current [80]:

$$
\left\langle P^{\prime}, \uparrow\left|\frac{J^{+}(0)}{2 P^{+}}\right| P, \uparrow\right\rangle=F_{1}\left(q^{2}\right)
$$

and

$$
\left\langle P^{\prime}, \uparrow\left|\frac{J^{+}(0)}{2 P^{+}}\right| P, \downarrow\right\rangle=-\frac{\left(q^{1}-i q^{2}\right)}{2 M} F_{2}\left(q^{2}\right) .
$$

On the higher dimensional gravity theory on the bulk, the spin-non-flip amplitude for the EM transition corresponds to the coupling of an external EM field $A^{M}(x, z)$ propagating in AdS with a fermionic mode $\Psi_{P}(x, z)$, given by the left-hand side of the

\footnotetext{
${ }^{15}$ See for example Ref. [284] and references therein.

${ }^{16}$ For a recent review see Ref. [303].
} 
equation below

$$
\begin{aligned}
\int d^{4} x d z \sqrt{g} \bar{\Psi}_{P^{\prime}}(x, z) e_{M}^{A} \Gamma_{A} A^{M}(x, z) \Psi_{P}(x, z) & \\
& \sim(2 \pi)^{4} \delta^{4}\left(P^{\prime}-P-q\right) \epsilon_{\mu} u\left(P^{\prime}\right) \gamma^{\mu} F_{1}\left(q^{2}\right) u(P),
\end{aligned}
$$

where $e_{M}^{A}=\left(\frac{R}{z}\right) \delta_{M}^{A}$ is the vielbein with curved space indices $M, N=0, \cdots 4$ and tangent indices $A, B=0, \cdots, 4$. The expression on the right-hand side represents the Dirac EM form factor in physical space-time. It is the EM spin-conserving matrix element (6.47) of the local quark current $J^{\mu}=e_{q} \bar{q} \gamma^{\mu} q$ with local coupling to the constituents. In this case one can also show that a precise mapping of the $J^{+}$elements can be carried out at fixed LF time, providing an exact correspondence between the holographic variable $z$ and the LF impact variable $\zeta$ in ordinary space-time with the result [138]

$$
G_{ \pm}\left(Q^{2}\right)=g_{ \pm} R^{4} \int \frac{d z}{z^{4}} V\left(Q^{2}, z\right) \Psi_{ \pm}^{2}(z)
$$

for the components $\Psi_{+}$and $\Psi_{-}$with angular momentum $L^{z}=0$ and $L^{z}=+1$ respectively. The effective charges $g_{+}$and $g_{-}$are determined from the spin-flavor structure of the theory.

A precise mapping for the Pauli form factor using light-front holographic methods has not been carried out. To study the spin-flip nucleon form factor $F_{2}$ (6.48) using holographic methods, Abidin and Carlson [180] propose to introduce a non-minimal electromagnetic coupling with the 'anomalous' gauge invariant term

$$
\int d^{4} x d z \sqrt{g} \bar{\Psi} e_{M}^{A} e_{N}^{B}\left[\Gamma_{A}, \Gamma_{B}\right] F^{M N} \Psi
$$

in the five-dimensional action, since the structure of (6.49) can only account for $F_{1}$. Although this is a practical avenue, the overall strength of the new term has to be fixed by the static quantities and thus some predictivity is lost.

Light-front holographic QCD methods have also been used to obtain hadronic momentum densities and generalized parton distributions (GPDs) of mesons and nucleons in the zero skewness limit [304, 305, 306, 307]. GPDs are nonperturbative, and thus holographic methods are well suited to explore their analytical structure ${ }^{17}$. LF holographic methods have been used to model transverse momentum dependent (TMD)

\footnotetext{
${ }^{17}$ A computation of GPDs and nucleon structure functions at small $x$ using gravity duals has been carried out in Refs. [308] and [309] respectively.
} 
parton distribution functions and fragmentation functions [310]. LF holography can also be used to study the flavor separation of the elastic nucleon form factors [311] which have been determined recently up to $Q^{2}=3.4 \mathrm{GeV}^{2}$ [312]. One can also use the holographic framework to construct light-front wave functions and parton distribution functions (PDFs) by matching quark counting rules [313]. Recently, models of nucleon and flavor form factors and GPDs has been discussed using LF holographic ideas and AdS/QCD [314, 315, 316, 317]. The Dirac and Pauli weak neutral nucleon form factors have also been examined using the framework of light-front holographic QCD in Ref. [318]. LF holography has also been used to describe nucleon transition form factors, such as $\gamma^{*} N \rightarrow N^{*}[319]^{18}$.

\subsubsection{Computing nucleon form factors in light-front holographic QCD}

In computing nucleon form factors we should impose the asymptotic boundary conditions by the leading fall-off of the form factors to match the twist of the hadron's interpolating operator, $i . \quad$ e. $\tau=3$, to represent the fact that at high energies the nucleon is essentially a system of 3 weakly interacting partons. However, as discussed at the end of Chapter 5, at low energies the strongly correlated bound state of $n$ quarks behaves as a system of an active quark vs. the $n-1$ spectators. This means, for example, that for a proton the nonperturbative bound state behaves as a quark-diquark system, $i$. e., a twist-2 system. In this simple picture, at large momentum transfer, or at small distances, where the cluster is resolved into its individual constituents, the baryon is governed by twist-3, whereas in the long-distance nonperturbative region by twist-2. Thus, at the transition region the system should evolve from twist-3 to twist- 2 . In practice, since the behavior of the form factors at very low energy is much constrained by its normalization, we will use a simple approximation where the nucleon form factor is twist-3 at all momentum transfer scales (In fact, twist-3 for the Dirac form factor, and twist-4 for the Pauli form factor to account for the $L=1$ orbital angular momentum). As in the case of the pion form factor described in the previous section, the vector-meson poles should be shifted to their physical locations for a meaningful comparison with data. With this limitations in mind, we describe below a simple model approximation to describe the space-like nucleon form factors.

\footnotetext{
${ }^{18} \mathrm{~A}$ computation of nucleon transition form factors has been carried out in the framework of the Sakai-Sugimoto model in Refs. [320] and [321]. Baryon form factors have also been computed using the SS framework in Refs. [322, 323].
} 
In order to compute the individual features of the proton and neutron form factors one needs to incorporate the spin-flavor structure of the nucleons, properties which are absent in models of the gauge/gravity correspondence. The spin-isospin symmetry can be readily included in light-front holography by weighting the different Fock-state components by the charges and spin-projections of the quark constituents; e.g., as given by the $S U(6)$ spin-flavor symmetry. We label by $N_{q \uparrow}$ and $N_{q \downarrow}$ the probability to find the constituent $q$ in a nucleon with spin up or down respectively. For the $S U(6)$ wave function [260] we have

$$
N_{u \uparrow}=\frac{5}{3}, \quad N_{u \downarrow}=\frac{1}{3}, \quad N_{d \uparrow}=\frac{1}{3}, \quad N_{d \downarrow}=\frac{2}{3},
$$

for the proton and

$$
N_{u \uparrow}=\frac{1}{3}, \quad N_{u \downarrow}=\frac{2}{3}, \quad N_{d \uparrow}=\frac{5}{3}, \quad N_{d \downarrow}=\frac{1}{3},
$$

for the neutron. The effective charges $g_{+}$and $g_{-}$in (6.50) are computed by the sum of the charges of the struck quark convoluted by the corresponding probability for the $L^{z}=0$ and $L^{z}=+1$ components $\Psi_{+}$and $\Psi_{-}$respectively. We find $g_{p}^{+}=1, g_{p}^{-}=0$, $g_{+}^{n}=-\frac{1}{3}$ and $g_{-}^{n}=\frac{1}{3}$. The nucleon Dirac form factors in the $S U(6)$ limit are thus given by

$$
\begin{aligned}
& F_{1}^{p}\left(Q^{2}\right)=R^{4} \int \frac{d z}{z^{4}} V\left(Q^{2}, z\right) \Psi_{+}^{2}(z) \\
& F_{1}^{n}\left(Q^{2}\right)=-\frac{1}{3} R^{4} \int \frac{d z}{z^{4}} V\left(Q^{2}, z\right)\left[\Psi_{+}^{2}(z)-\Psi_{-}^{2}(z)\right],
\end{aligned}
$$

where $F_{1}^{p}(0)=1$ and $F_{1}^{n}(0)=0$.

In the soft-wall model the plus and minus components of the leading twist-3 nucleon wave function are

$$
\Psi_{+}(z)=\frac{\sqrt{2} \kappa^{2}}{R^{2}} z^{7 / 2} e^{-\kappa^{2} z^{2} / 2}, \quad \Psi_{-}(z)=\frac{\kappa^{3}}{R^{2}} z^{9 / 2} e^{-\kappa^{2} z^{2} / 2},
$$

where we have absorbed the dilaton exponential dependence by a redefinition of the AdS wave function, and the bulk-to-boundary propagator $V\left(Q^{2}, z\right)$ is given by (6.28). The results for $F_{1}^{p, n}$ follow from the analytic form (6.37) for any twist $\tau$. We find

$$
F_{1}^{p}\left(Q^{2}\right)=F_{+}\left(Q^{2}\right)
$$



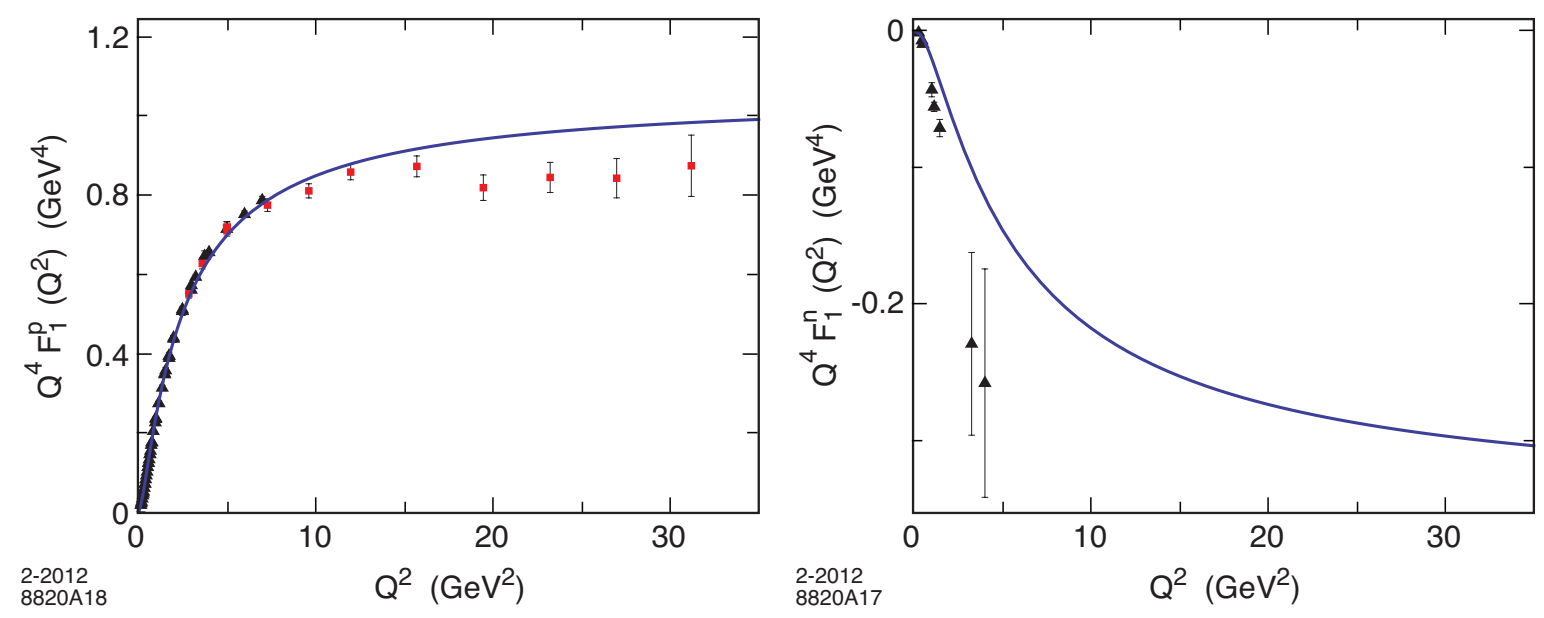

Figure 6.4: Light-front holographic predictions for $Q^{4} F_{1}^{p}\left(Q^{2}\right)$ (left) and $Q^{4} F_{1}^{n}\left(Q^{2}\right)$ (right) in the $S U(6)$ limit. Data compilation from Diehl [269].

and

$$
F_{1}^{n}\left(Q^{2}\right)=-\frac{1}{3}\left(F_{+}\left(Q^{2}\right)-F_{-}\left(Q^{2}\right)\right)
$$

where we have, for convenience, defined the twist-3 and twist-4 form factors

$$
F_{+}\left(Q^{2}\right)=\frac{1}{\left(1+\frac{Q^{2}}{\mathcal{M}_{\rho}^{2}}\right)\left(1+\frac{Q^{2}}{\mathcal{M}_{\rho^{\prime}}^{2}}\right)}
$$

and

$$
F_{-}\left(Q^{2}\right)=\frac{1}{\left(1+\frac{Q^{2}}{\mathcal{M}_{\rho}^{2}}\right)\left(1+\frac{Q^{2}}{\mathcal{M}_{\rho^{\prime}}^{2}}\right)\left(1+\frac{Q^{2}}{\mathcal{M}_{\rho^{\prime \prime}}^{2}}\right)},
$$

with the multiple pole structure derived from the soft-wall dressed EM current propagating in AdS space. The results for $Q^{4} F_{1}^{p}\left(Q^{2}\right)$ and $Q^{4} F_{1}^{n}\left(Q^{2}\right)$ are shown in Fig. 6.4. The value $\sqrt{\lambda}=0.548 \mathrm{GeV}$ is determined from the $\rho$ mass.

The expression for the elastic nucleon form factor $F_{2}^{p, n}$ follows from (6.46) and (6.51).

$$
F_{2}^{p, n}\left(Q^{2}\right) \sim \int \frac{d z}{z^{3}} \Psi_{+}(z) V\left(Q^{2}, z\right) \Psi_{-}(z)
$$

Using the twist-3 and twist-4 nucleon soft-wall wave functions $\Psi_{+}$and $\Psi_{-}$(6.56) we find

$$
F_{2}^{p, n}\left(Q^{2}\right)=\chi_{p, n} F_{-}\left(Q^{2}\right)
$$



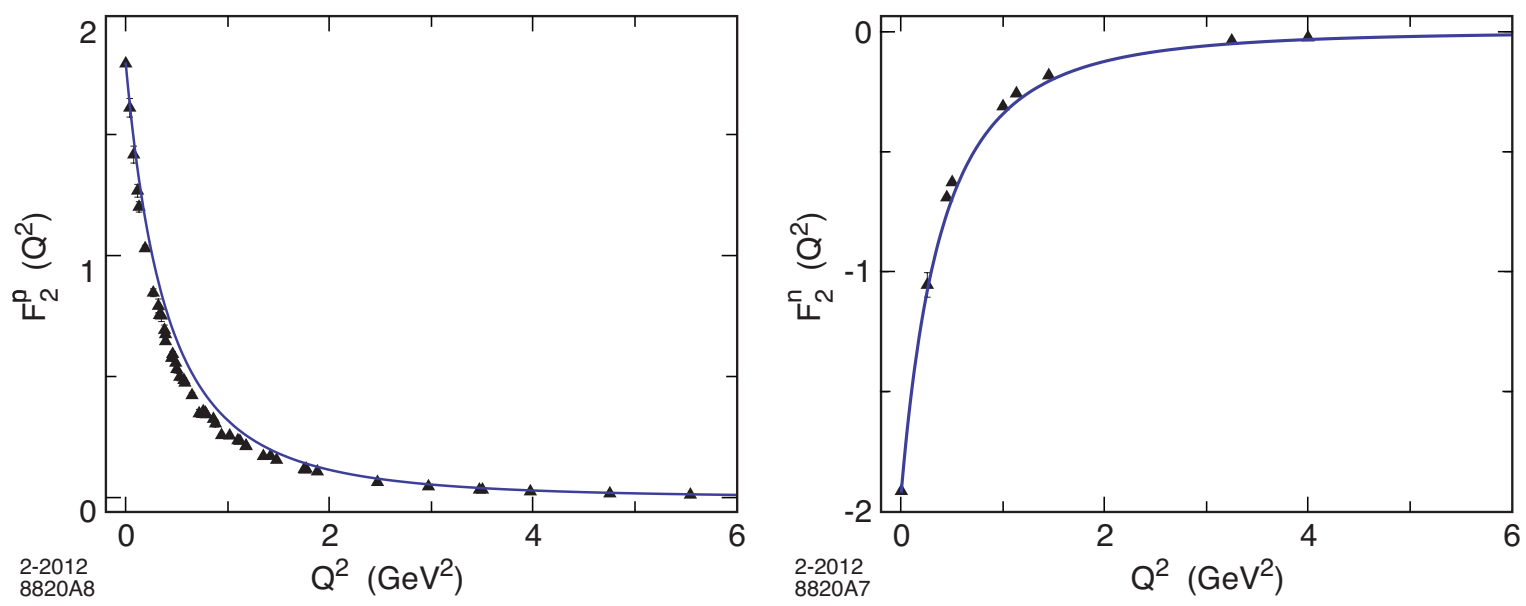

Figure 6.5: Light-front holographic predictions for $F_{2}^{p}\left(Q^{2}\right)$ (left) and $F_{2}^{n}\left(Q^{2}\right)$ (right). The value of the nucleon anomalous magnetic moment $\chi$ is taken from experiment. Data compilation from Diehl [269].

where the amplitude (6.61) has been normalized to the static quantities $\chi_{p}$ and $\chi_{n}$ and $F_{-}\left(Q^{2}\right)$ is given by (6.60). The experimental values $\chi_{p}=1.793$ and $\chi_{n}=-1.913$ are consistent with the $S U(6)$ prediction [324] $\mu_{P} / \mu_{N}=-3 / 2$. In fact $\left(\mu_{P} / \mu_{N}\right)_{\exp }=-1.46$, where $\mu_{P}=1+\chi_{p}$ and $\mu_{N}=\chi_{n}$. The results for $F_{2}^{p}\left(Q^{2}\right)$ and $F_{2}^{n}\left(Q^{2}\right)$ are shown in Fig. 6.5. The vector meson masses are given by $M^{2}=4 \lambda\left(n+\frac{1}{2}\right)$ with the value $\sqrt{\lambda}=0.548$ GeV obtained from the $\rho$ mass.

We compute the charge and magnetic root-mean-square (rms) radius from the usual electric and magnetic nucleon form factors

$$
G_{E}\left(q^{2}\right)=F_{1}\left(q^{2}\right)+\frac{q^{2}}{4 \mathcal{M}^{2}} F_{2}\left(q^{2}\right)
$$

and

$$
G_{M}\left(q^{2}\right)=F_{1}\left(q^{2}\right)+F_{2}\left(q^{2}\right)
$$

Using the definition

$$
\left\langle r^{2}\right\rangle=-\left.\frac{6}{F(0)} \frac{d F\left(Q^{2}\right)}{d Q^{2}}\right|_{Q^{2}=0}
$$

we find the values $\sqrt{\left\langle r_{E}\right\rangle_{p}}=0.802 \mathrm{fm}, \sqrt{\left\langle r_{M}^{2}\right\rangle_{p}}=0.758 \mathrm{fm},\left\langle r_{E}^{2}\right\rangle_{n}=-0.10 \mathrm{fm}^{2}$ and $\sqrt{\left\langle r_{M}^{2}\right\rangle_{n}}=0.768 \mathrm{fm}$, compared with the experimental values $\sqrt{\left\langle r_{E}\right\rangle_{p}}=(0.877 \pm$ $0.007) \mathrm{fm}, \sqrt{\left\langle r_{M}^{2}\right\rangle_{p}}=(0.777 \pm 0.016) \mathrm{fm},\left\langle r_{E}^{2}\right\rangle_{n}=(-0.1161 \pm 0.0022) \mathrm{fm}^{2}$ and $\sqrt{\left\langle r_{M}^{2}\right\rangle_{n}}=$ 
$(0.862 \pm 0.009) \mathrm{fm}$ from electron-proton scattering experiments [16]. ${ }^{19}$ The muonic hydrogen measurement gives $\sqrt{\left\langle r_{E}\right\rangle_{p}}=0.84184(67) \mathrm{fm}$ from Lamb-shift measurements [325]. ${ }^{20}$

Chiral effective theory predicts that the slopes of the form factors are singular for zero pion mass. For example, the slope of the Pauli form factor of the proton at $q^{2}=0$ computed by Beg and Zepeda diverges as $1 / m_{\pi}$ [326]. This result comes from the triangle diagram $\gamma^{*} \rightarrow \pi^{+} \pi^{-} \rightarrow p \bar{p}$. One can also argue from dispersion theory that the singular behavior of the form factors as a function of the pion mass comes from the two-pion cut. Lattice theory computations of nucleon form factors require a strong dependence at small pion mass to extrapolate the predictions to the physical pion mass [327]. The two-pion calculation [326] is a Born computation which probably does not exhibit vector dominance. To make a reliable computation in the hadronic basis of intermediate states one evidently has to include an infinite number of states. On the other hand, divergences do not appear in light-front holographic QCD even for massless pions when we use the dressed current. In fact, the holographic analysis with a dressed EM current in AdS generates a nonperturbative multi-vector meson pole structure. ${ }^{21}$

\footnotetext{
${ }^{19}$ The neutron charge radius is defined by $\left\langle r_{E}^{2}\right\rangle_{n}=-\left.6 \frac{d G_{E}\left(Q^{2}\right)}{d Q^{2}}\right|_{Q^{2}=0}$.

${ }^{20}$ Other soft and hard-wall model predictions of the nucleon rms radius are given, for example, in Refs. [180, 306, 307].

${ }^{21}$ In the case of a free propagating current in AdS, we obtain logarithmic divergent results in the chiral limit.
} 


\section{Chapter 7}

\section{Other Bottom-Up and Top-Down Holographic Models}

Here we review some of the other holographic approaches to hadronic physics, and after discussing generic features of these models we focus on particular top-down and bottom-up models based on chiral symmetry breaking. Top-down models are derived from brane configurations in string theory, while bottom-up models, like Light Front Holographic QCD, are more phenomenological and are not restricted by the constraints of string theory-based models. All holographic QCD models include degrees of freedom which are identified with Standard Model hadrons via their quantum numbers, and predictions of QCD observables may be compared to experiment and to other models, often with remarkable quantitative success accurate to within the 10-15\% level.

By now there are enough examples of gauge theories with established gravity duals that a dictionary exists between features of the gauge theory and corresponding properties of the higher-dimensional gravity dual. The AdS/CFT correspondence relates operators in the lower-dimensional theory to fields in the higher-dimensional dual theory [22]. The quantum numbers and conformal dimensions of the gauge theory operators dictate the nature of the corresponding fields [27, 28]. Global symmetries in the $3+1$ dimensional theory become gauge invariances of the $4+1$ dimensional theory. Hence, symmetry currents are related to gauge fields in the gravity dual. In the case of $\mathcal{N}=4$ Yang-Mills theory, the $S O(6) R$-symmetry, which is a global symmetry of the theory, is associated with the $S O(6)$ isometry of the five-sphere in the $\operatorname{AdS}_{5} \times S^{5}$ supergravity background. A Kaluza-Klein decomposition of the gravitational fluctuations on the 5-sphere include spin-1 $S O(6)$ gauge fields in the effective $4+1$ dimensional theory on $\mathrm{AdS}_{5}$. In addition to $R$-symmetries, there may be flavor symmetries due to the ad- 
dition of probe branes [328], on which gauge fields propagate as fluctuations of open strings ending on the probe branes. The $3+1$ dimensional interpretation of the theory follows either from the behavior of the solutions to the equations of motion near the boundary of $\mathrm{AdS}_{5}$, or from a further Kaluza-Klein decomposition of the $4+1$ dimensional theory on $\mathrm{AdS}_{5}$. The boundary conditions do not allow massless Kaluza-Klein modes of the gauge fields to propagate, so the gauge invariance is absent in the $3+1$ dimensional effective theory while a global symmetry typically remains. In AdS/QCD models, isospin is the remnant of a $4+1$ dimensional gauge invariance with the same gauge group as the isospin symmetry group. The notion that boundary conditions in an extra dimension may be responsible for breaking of gauge invariances is also the basis of Higgsless models of electroweak symmetry breaking [329] and holographic technicolor models [330, 331, 332].

In order to describe a confining theory the conformal invariance encoded in the isometries of $\mathrm{AdS}_{5}$ must be broken. In bottom-up models the breaking of conformal symmetry is most easily modeled by way of a hard wall [32], so that the space-time geometry becomes a slice of $\mathrm{AdS}_{5}$. The location of the hard wall determines the typical scale of hadronic masses in hard-wall AdS/QCD models. While phenomenological AdS/QCD models often take the slice of $\mathrm{AdS}_{5}$ as the background space-time, in models based on string theory the space-time geometry is dictated by the corresponding brane configuration and may be quite different from $\operatorname{AdS}_{5}[53,38,39]$. A comparison of the predictions of AdS/QCD models in various space-time backgrounds appears in Ref. [54]. The result of such a comparison is that, for a wide class of space-time geometries, naive predictions of the classical 5D models (ignoring quantum corrections) agree with experiment at the 10-30\% level, with the slice of $\mathrm{AdS}_{5}$ often producing among the most accurate predictions. It is not known whether there is an underlying reason for the phenomenological success of models based on the AdS geometry, though there are arguments based on decoupling of high dimension operators at low energies [333] and suggestions of scale invariance in QCD at low energies [46] that may help to understand this.

\subsection{Bottom-up models}

Bottom-up holographic QCD models, like Light-Front Holographic QCD described in previous chapters, are loosely related to string-theory examples of holographic dualities, but are phenomenological and not constrained by the restrictions of string theory. 
The flexibility in bottom-up model building allows for matching of various aspects of QCD, for example the pattern of explicit and spontaneous chiral symmetry breaking and certain aspects of QCD at high energies (e.g. operator product expansions [69, 334]). Bottom-up models are motivated by the AdS/CFT dictionary relating properties of a non-gravitational theory to a gravitational theory in one additional dimension. Interpolating operators for QCD states map into fields in the higher-dimensional model. For example, as described in earlier sections, the rho mesons are created by the $S U(2)$ vector isospin current, so in order to model the spectrum of rho mesons the higher-dimensional model would include a field with the appropriate quantum numbers to couple to the vector current, namely an $S U(2)$ gauge field. For simplicity, and motivated by conformal symmetry, the higher-dimensional space-time in bottom-up holographic models is typically chosen to be Anti-de Sitter space, with metric that we repeat here:

$$
d s^{2}=\frac{R^{2}}{z^{2}}\left(\eta_{\mu \nu} d x^{\mu} d x^{\nu}-d z^{2}\right)
$$

where the $z$ coordinate describes the extra spatial dimension. In order to break the conformal symmetry the AdS space-time may be chosen to end at some value of $z$, as in hard-wall models, or else some field may have a non-vanishing background with a dimensionful parameter, as in the soft-wall model. Both hard-wall and soft-wall models maintain the $3+1$ dimensional Poincaré invariance of the space-time.

Consider an $S U(2)$ gauge theory on the slice of $\mathrm{AdS}_{5}$ with metric Eq. (7.1) between the boundary at $z=0$ and an infrared cutoff at $z=z_{m}$. The 5 -dimensional $S U(2)$ gauge fields are related via the AdS/CFT correspondence to a 4-dimensional $S U(2)$ current which may be identified with the isospin current of QCD. The action for this theory is

$$
\begin{aligned}
S & =-\frac{1}{4 g_{5}^{2}} \int d^{4} x d z \sqrt{g} F_{M N}^{a} F_{P Q}^{a} g^{M P} g^{N Q} \\
& =-\frac{1}{4 g_{5}^{2}} \int d^{4} x d z \frac{R}{z}\left(F_{\mu \nu}^{a} F_{\rho \sigma}^{a} \eta^{\mu \rho} \eta^{\nu \sigma}-2 F_{\mu z}^{a} F_{\nu z}^{a} \eta^{\mu \nu}\right) \\
& =-\frac{1}{4 g_{5}^{2}} \int d^{4} x d z \frac{R}{z}\left(F_{\mu \nu}^{a} F^{a \mu \nu}+2 F_{z \mu}^{a} F^{a} z \mu\right),
\end{aligned}
$$

where $F_{M N}^{a}=\partial_{M} V_{N}^{a}-\partial_{N} V_{M}^{a}+\epsilon^{a b c} V_{M}^{b} V_{N}^{c}$ is the field strength tensor for the gauge fields $V_{M}^{a}, g_{5}$ is the 5-dimensional gauge coupling, and the $S U(2)$ gauge index $a$ runs from 1 to 3. As usual, Greek indices are contracted with the 4-dimensional Minkowski tensor $\eta_{\mu \nu}$ as in the last line of Eq. (7.2), while capital Latin indices run from 0 to 4 and contractions of capital Latin indices with $g_{M N}$ are made explicit so as to avoid confusion. 
The linearized equations of motion are

$$
\begin{gathered}
\frac{1}{z} \partial_{\mu} F^{a \mu \nu}+\partial_{z}\left(\frac{1}{z} F^{a z \nu}\right)=0, \\
\frac{1}{z} \partial_{\mu} F^{a \mu z}=0
\end{gathered}
$$

where for the present discussion the field strengths are to be linearized. Note that $R$ and $g_{5}$ factor out of the linearized equations of motion, and otherwise they appear in the dimensionless combination $R / g_{5}^{2}$. At the infrared boundary $z=z_{m}$ there is freedom in the choice of boundary conditions. We will impose the simplest gaugeinvariant boundary conditions, $F_{\mu z}^{a}\left(x, z_{m}\right)=0$. The Kaluza-Klein modes must also be normalizable as $z \rightarrow 0$, i.e. the integral over $z$ in the action Eq. (7.2) must remain finite upon replacing the gauge fields by a Kaluza-Klein mode. As we will review, a Weinberg sum rule is an automatic consequence of the choice of Dirichlet boundary conditions on the gauge fields in a UV-regulated boundary at some $z=\epsilon$, where $\epsilon \rightarrow 0$.

Suppose we fix a gauge $V_{z}^{a}=0$. In that case the linearized equations of motion become,

$$
\frac{1}{z} \partial_{\mu} F^{a \mu \nu}-\partial_{z}\left(\frac{1}{z} \partial_{z} V^{a \nu}\right)=0
$$

together with a transverseness condition $\partial_{z} \partial_{\mu} V^{a \mu}=0$. From the transverseness condition follows that $\partial_{\mu} V^{a \mu}$ is independent of $z$, and then by a $z$-independent gauge transformation condition consistent with our choice $V^{a z}=0$, we may also set $\partial_{\mu} V^{a \mu}=0$. The 4-dimensional-transverse Kaluza-Klein modes are solutions to the linearized equations of motion of the form

$$
V_{n}^{a \mu}(x, z)=\varepsilon_{n}^{a \mu} e^{i q \cdot x} \psi_{n}(z),
$$

where $q^{2}=m_{n}^{2}, q \cdot \varepsilon^{a}=0$. The wavefunction $\psi_{n}(z)$ then satisfies the equation

$$
\frac{d}{d z}\left(\frac{1}{z} \frac{d \psi_{n}(z)}{d z}\right)+\frac{m_{n}^{2}}{z} \psi_{n}(z)=0
$$

with boundary conditions $\psi^{\prime}\left(z_{m}\right)=0$ and normalizability, with normalization

$$
\int_{\epsilon}^{z_{m}} d z \psi_{n}(z)^{2} / z=1
$$

The subscript $n$ denotes the discrete set of eigenvalues $m_{n}^{2}$ of the Sturm-Liouville system. In practice we impose Dirichlet $\left(\psi_{n}(\epsilon)=0\right)$ boundary conditions on the modes at an 
unphysical ultraviolet cutoff $z=\epsilon \ll z_{m}$. As $\epsilon \rightarrow 0$ the spectrum becomes independent of the specific form of the Sturm-Liouville boundary condition except for the addition of a zero mode in the Neumann case. The extra zero mode decouples from the rest of the spectrum in the $\epsilon \rightarrow 0$ limit, as can be seen from the fact that the integral over $z$ in the action for the zero mode diverges in this limit.

The equations of motion Eq. (7.5) imply that the Kaluza-Klein modes satisfy the Proca equation,

$$
\partial_{\mu} F^{a \mu \nu}=-m_{n}^{2} V^{a \nu}
$$

so the eigenvalue $m_{n}$ is identified with the mass of the corresponding $3+1$ dimensional field.

By construction the Kaluza-Klein modes have quantum numbers conjugate to the corresponding $3+1$ dimensional current $J_{\mu}^{a}=\bar{q} \gamma^{\mu} T^{a} q$, where $T^{a}$ are the $S U(2)$ isospin generators normalized such that $\operatorname{Tr} T^{a} T^{b}=\frac{1}{2} \delta^{a b}$. Both the gauge fields $V_{\mu}^{a}$ and the current $J_{\mu}^{a}$ transform in the adjoint representation of $S U(2)$. Under charge conjugation $V_{\mu}^{a}$ is odd, and parity acts as $V_{i}^{a}(t, \mathbf{x}, z) \rightarrow-V_{i}^{a}(t,-\mathbf{x}, z)$, while $V_{0}^{a}$ and $V_{z}^{a}$ are even under parity. These charge assignments identify the Kaluza-Klein modes with states obtained by acting with the current $J_{\mu}^{a}$ on the vacuum.

A useful object to consider is the bulk-to-boundary propagator, which is related to the solution of the equations of motion subject to the boundary condition $V^{a \mu}(x, z) \rightarrow$ $V_{0}^{a \mu}(x)$ as $z \rightarrow 0$ for arbitrary fixed $V_{0}^{a \mu}(x)[27,28]$. We have already encountered this object in Chapter 6, where it was used in the calculation of meson and nucleon electromagnetic form factors. The boundary profile of the field, $V_{0}^{a \mu}(x)$, plays the role of the source of the current $J_{\mu}^{a}(x)$ in the AdS/CFT correspondence. Fourier transforming in $3+1$ dimensions, with a slight abuse of notation we equivalently have $V^{a \mu}(q, z) \rightarrow V_{0}^{a \mu}(q)$ as $z \rightarrow 0$. With

$$
V^{a \mu}(x, z)=\int \frac{d^{4} q}{(2 \pi)^{4}} e^{-i q \cdot x} V_{0}^{a \mu}(q) V(q, z),
$$

the bulk-to-boundary propagator $V(q, z)$ satisfies,

$$
\partial_{z}\left(\frac{1}{z} \partial_{z} V(q, z)\right)+\frac{q^{2}}{z} V(q, z)=0,
$$

with $V(q, \epsilon)=1$ and $\left.\partial_{z} V(q, z)\right|_{z=\epsilon}=0$. The bulk-to-boundary propagator determines solutions to the equations of motion that have a prescribed form on the UV boundary of the space-time, $z=\epsilon$.

The bulk-to-boundary propagator can be decomposed in terms of the normaliz- 
able eigensolutions as follows [274]: Define the Green function $G\left(q, z, z^{\prime}\right)$ with Dirichlet boundary conditions at $z=\epsilon$,

$$
\begin{gathered}
\left(\partial_{z} \frac{1}{z} \partial_{z}+\frac{q^{2}}{z}\right) G\left(q, z, z^{\prime}\right)=\delta\left(z-z^{\prime}\right), \\
G\left(q, \epsilon, z^{\prime}\right)=0,\left.\quad \partial_{z} G\left(q, z, z^{\prime}\right)\right|_{z=z_{m}}=0 .
\end{gathered}
$$

Now consider the integral

$$
\int_{\epsilon}^{z_{m}} d z V(q, z)\left(\partial_{z} \frac{1}{z} \partial_{z}+\frac{q^{2}}{z}\right) G\left(q, z, z^{\prime}\right)=V\left(q, z^{\prime}\right) .
$$

Integrating by parts twice, we have,

$$
\begin{aligned}
V\left(q, z^{\prime}\right)= & \int_{\epsilon}^{z_{m}} d z G\left(q, z, z^{\prime}\right)\left(\partial_{z} \frac{1}{z} \partial_{z}+\frac{q^{2}}{z}\right) V(q, z) \\
& +\left.\left(V(q, z) \frac{1}{z} \partial_{z} G\left(q, z, z^{\prime}\right)-G\left(q, z, z^{\prime}\right) \frac{1}{z} \partial_{z} V(q, z)\right)\right|_{z=\epsilon} ^{z_{m}} \\
= & -\left.\frac{1}{z} \partial_{z} G\left(q, z, z^{\prime}\right)\right|^{z=\epsilon} .
\end{aligned}
$$

By Eqs.(7.12) and (7.13), the Green function can be decomposed in the complete set of normalizable solutions $\psi_{n}(z)$ defined earlier:

$$
G\left(q, z, z^{\prime}\right)=\sum_{n} \frac{\psi_{n}(z) \psi_{n}\left(z^{\prime}\right)}{q^{2}-m_{n}^{2}}
$$

where the wavefunctions are normalized as in Eq. (7.8). Hence, we obtain

$$
V\left(q, z^{\prime}\right)=-\sum_{n} \frac{\psi_{n}^{\prime}(\epsilon)}{\epsilon} \frac{\psi_{n}\left(z^{\prime}\right)}{q^{2}-m_{n}^{2}}
$$

as long as $z^{\prime} \neq \epsilon$. A consequence of the hard-wall boundary condition on the KaluzaKlein modes is the Gibbs phenomenon at $z^{\prime}=\epsilon$, which is the discontinuity in the Fourier series of a function with a jump discontinuity. In our case the discontinuity can be seen by comparing Eq. (7.17) with $\psi_{n}(\epsilon)=0$ and the condition $V(q, \epsilon)=1$. However, limits as $z^{\prime} \rightarrow \epsilon$ are well defined. This situation is analogous to the problem in Fourier transforming a square waveform in terms of modes which vanish at the boundaries of the square. In that case, the Fourier transform strictly vanishes at the discontinuity, 
and for any sum over a finite number of modes there is a deviation in the function from the desired waveform in the neighborhood of the discontinuity.

In the context of the hard-wall model, the precise statement of the AdS/CFT correspondence in the gravity limit is that the generating functional of connected correlation functions of products of currents, $W\left[V_{0}^{a \mu}(x)\right]$ in $3+1$ physical space-time, is identified with the $4+1$ dimensional action $S$ evaluated on solutions to the equations of motion such that $V^{a \mu}(x, z) \rightarrow V_{0}^{a \mu}(x)$ as $z \rightarrow \epsilon[27,28,175]$. The gravity limit is the limit of infinite $N$ and large 't Hooft coupling [22], but in the context of the hard-wall model the same analysis is equivalent to studying the effective $3+1$ dimensional theory derived from the $4+1$ dimensional model in the classical limit, i.e. having absorbed quantum corrections into the definition of the model.

Integrating the action by parts, the action vanishes by the equations of motion except for a boundary term at $z=\epsilon$. Hence, the generating functional has the form,

$$
\begin{aligned}
& W\left[V_{0}^{a \mu}(x)\right]=S\left[V_{0}^{a \mu}(x)\right]=-\left.\frac{R}{2 g_{5}^{2}} \int d^{4} x\left[V^{a \mu}(x, z)\left(g_{\mu \nu}-\frac{\partial_{\mu} \partial_{\nu}}{\partial^{2}}\right) \frac{1}{z} \partial_{z} V^{a \nu}(x, z)\right]\right|_{z=\epsilon}+\ldots \\
& =-\left.\frac{R}{2 g_{5}^{2}} \int d^{4} x \int \frac{d^{4} \bar{q}}{(2 \pi)^{4}} \frac{d^{4} \bar{q}^{\prime}}{(2 \pi)^{4}}\left[V_{0}^{a \mu}\left(\bar{q}^{\prime}\right) e^{-i\left(\bar{q}^{\prime}+\bar{q}\right) \cdot x} V\left(\bar{q}^{\prime}, z\right)\left(g_{\mu \nu}-\frac{\bar{q}_{\mu} \bar{q}_{\nu}}{\bar{q}^{2}}\right) V_{0}^{a \nu}(\bar{q}) \frac{1}{z} \partial_{z} V(\bar{q}, z)\right]\right|_{z=\epsilon}+\ldots \\
& =-\left.\frac{R}{2 g_{5}^{2}} \int \frac{d^{4} \bar{q}}{(2 \pi)^{4}}\left[V_{0}^{a \mu}(-\bar{q})\left(g_{\mu \nu}-\frac{\bar{q}_{\mu} \bar{q}_{\nu}}{\bar{q}^{2}}\right) V^{a \nu}(\bar{q}) \frac{1}{\epsilon} \partial_{z} V(\bar{q}, z)\right]\right|_{z=\epsilon}+\ldots \\
& =-\left.\frac{R}{2 g_{5}^{2}} \int \frac{d^{4} \bar{q}}{(2 \pi)^{4}} \int d^{4} \bar{x} d^{4} \bar{x}^{\prime}\left[e^{i \bar{q} \cdot\left(\bar{x}-\bar{x}^{\prime}\right)} V_{0}^{a \mu}\left(\bar{x}^{\prime}\right)\left(g_{\mu \nu}-\frac{\bar{q}_{\mu} \bar{q}_{\nu}}{\bar{q}^{2}}\right) V_{0}^{a \nu}(\bar{x}) \frac{1}{z} \partial_{z} V(\bar{q}, z)\right]\right|_{z=\epsilon}+\ldots,
\end{aligned}
$$

where the ellipsis represents terms more than quadratic in $V_{0}^{a \mu}$. The transverse projection operator enforces the transverseness of field-theory correlators derived from this generating functional.

The AdS/CFT prediction for the current-current correlator follows:

$$
\begin{aligned}
\left\langle J_{\mu}^{a}(x) J_{\nu}^{b}(0)\right\rangle & =\frac{\delta^{2} W}{\delta V^{a \mu}(x) \delta V^{b \nu}(0)} \\
& =-\left.\delta^{a b} \frac{R}{g_{5}^{2}} \int \frac{d^{4} \bar{q}}{(2 \pi)^{4}} \int d^{4} \bar{x} d^{4} \bar{x}^{\prime} e^{i \bar{q} \cdot\left(\bar{x}^{\prime} \bar{x}^{\prime}\right)} \delta^{4}\left(\bar{x}^{\prime}-x\right)\left(g_{\mu \nu}-\frac{\bar{q}_{\mu} \bar{q}_{\nu}}{\bar{q}^{2}}\right) \delta^{4}(\bar{x}) \frac{1}{z} \partial_{z} V(\bar{q}, z)\right|_{z=\epsilon} \\
& =-\left.\delta^{a b} \frac{R}{g_{5}^{2}} \int \frac{d^{4} \bar{q}}{(2 \pi)^{4}} e^{-i \bar{q} \cdot x}\left(g_{\mu \nu}-\frac{\bar{q}_{\mu} \bar{q}_{\nu}}{\bar{q}^{2}}\right) \frac{1}{z} \partial_{z} V(\bar{q}, z)\right|_{z=\epsilon}
\end{aligned}
$$


Fourier transforming,

$$
\int d^{4} x e^{i q \cdot x}\left\langle J_{\mu}^{a}(x) J_{\nu}^{b}(0)\right\rangle=-\left.\delta^{a b} \frac{R}{g_{5}^{2}}\left(g_{\mu \nu}-\frac{q_{\mu} q_{\nu}}{q^{2}}\right) \frac{1}{z} \partial_{z} V(q, z)\right|_{z=\epsilon} .
$$

Expressing the bulk-to-boundary propagator $V(\bar{q}, z)$ in terms of the Kaluza-Klein modes as in Eq. (7.17), we obtain the decomposition

$$
\int d^{4} x e^{i q \cdot x}\left\langle J_{\mu}^{a}(x) J_{\nu}^{b}(0)\right\rangle=\delta^{a b} \frac{R}{g_{5}^{2}}\left(g_{\mu \nu}-\frac{q_{\mu} q_{\nu}}{q^{2}}\right) \lim _{z \rightarrow \epsilon} \sum_{n} \frac{\left(\psi_{n}^{\prime}(\epsilon) / \epsilon\right)\left(\psi_{n}^{\prime}(z) / z\right)}{q^{2}-m_{n}^{2}} .
$$

The polarization $\Pi_{V}\left(q^{2}\right)$ is defined in terms of the two-point function of currents via

$$
\int d^{4} x e^{i q \cdot x}\left\langle J_{\mu}^{a}(x) J_{\nu}^{b}(0)\right\rangle=-\delta^{a b}\left(g_{\mu \nu} q^{2}-q_{\mu} q_{\nu}\right) \Pi_{V}\left(q^{2}\right)
$$

so we identify

$$
\begin{aligned}
\Pi_{V}\left(q^{2}\right) & =\left.\frac{R}{g_{5}^{2}} \frac{1}{q^{2}} \frac{\partial_{z} V(q, z)}{z}\right|_{z=\epsilon} \\
& =-\frac{R}{g_{5}^{2}} \lim _{z \rightarrow \epsilon} \sum_{n} \frac{\left(\psi_{n}^{\prime}(\epsilon) / \epsilon\right)\left(\psi_{n}^{\prime}(z) / z\right)}{q^{2}\left(q^{2}-m_{n}^{2}\right)} \\
& =-\frac{R}{g_{5}^{2}} \sum_{n} \frac{\left[\psi_{n}^{\prime}(\epsilon) / \epsilon\right]^{2}}{m_{n}^{2}\left(q^{2}-m_{n}^{2}\right)}
\end{aligned}
$$

up to a contact interaction from the replacement of the factor of $1 / q^{2}$ in Eq. (7.24) with $1 / m_{n}^{2}$ in Eq. (7.25).

The analytic solution for $V(q, z)$ as the regulator $\epsilon \rightarrow 0$ is

$$
V(q, z)=\frac{\pi q z}{2 J_{0}\left(q z_{m}\right)}\left(J_{1}(q z) Y_{0}\left(q z_{m}\right)-Y_{1}(q z) J_{0}\left(q z_{m}\right)\right) .
$$

so we obtain an analytic solution for the polarization $\Pi_{V}[335,330,70]$,

$$
\Pi_{V}\left(q^{2}\right)=-\frac{\pi R\left(J_{0}\left(q z_{m}\right) Y_{0}(q \epsilon)-Y_{0}\left(q z_{m}\right) J_{0}(q \epsilon)\right)}{2 g_{5}^{2} J_{0}\left(q z_{m}\right)} .
$$

The rho masses $m_{\rho_{n}}$ are determined by the poles of $\Pi_{V}\left(q^{2}\right)$, at which

$$
J_{0}\left(m_{\rho_{n}} z_{m}\right)=0 .
$$


Analytically continuing the bulk-to-boundary propagator $V(q, z)$ in $q$ and expanding for large Euclidean momentum, $-q^{2} \gg 1$, near the boundary of $\mathrm{AdS}_{5}$,

$$
V(q, z)=1+\frac{\left(-q^{2} z^{2}\right)}{4} \log \left(-q^{2} z^{2}\right)+\ldots
$$

and

$$
\Pi_{V}\left(q^{2}\right)=-\frac{R}{2 g_{5}^{2}} \log \left(-q^{2} \epsilon^{2}\right)+\text { const.. }
$$

This is the correct logarithmic behavior for the current-current correlator in the conformal theory. The identification of Eqs. (7.30) and (7.25) is reminiscent of a Weinberg sum rule. The sum over Kaluza-Klein modes in Eq. (7.25) can be identified with a sum over resonances $\rho_{n}^{a \mu}$ carrying the quantum numbers of the current $J_{\mu}^{a}$. With this identification, we can read off the decay constants $F_{n}$ such that $\left\langle 0\left|J_{\mu}^{a}(0)\right| \rho_{n}^{b}\right\rangle=F_{n} \delta^{a b} \varepsilon_{\mu}$ for a resonance with transverse polarization $\varepsilon_{\mu}$ :

$$
F_{n}^{2}=\frac{R}{g_{5}^{2}}\left[\psi_{n}^{\prime}(\epsilon) / \epsilon\right]^{2}
$$

If we choose to match this result to the perturbative QCD result $\Pi_{V}\left(q^{2}\right) \approx-\frac{N_{c}}{24 \pi^{2}} \log \left(-q^{2}\right)$ as in Ref. [69, 70], we then identify

$$
g_{5}^{2}=\frac{12 \pi^{2}}{N_{c}}
$$

If $z_{m}$ is chosen so that $m_{\rho}=776 \mathrm{MeV}$, then the model predicts the decay constant for the rho to be $F_{\rho}^{1 / 2}=329 \mathrm{MeV}$ [69], to be compared to the experimental value of $345 \pm 8$ $\mathrm{MeV}$ [16]. In principle this model predicts properties of an infinite spectrum of radial excitations of the rho, though the model also ignores all resonances other than these, and it is not surprising that predictions are inaccurate at scales significantly higher than the mass of the lightest rho meson.

A generic feature of holographic models based on classical equations of motion is that he Kaluza-Klein resonances are infinitely narrow, as can be seen in this example by the fact that the poles in $\Pi_{V}$ are at real values of $q^{2}$. The classical limit, or supergravity limit, of the AdS/CFT correspondence is a large- $N_{C}$ limit of the gauge theory. The width of mesonic resonances in QCD goes to zero as the number of colors goes to infinity, so this feature of holographic models may be considered a remnant of the large- $N_{C}$ limit, even as $N_{C}$ is set to 3 in results such as Eq. (7.32). In more elaborate models, couplings 
of the rho meson to pions can be calculated, from which the width of the rho meson can be inferred. This begins a bootstrap approach to holographic model building in which quantum corrections are self-consistently included. This approach has not been elaborated on in the literature.

\subsection{A bottom-up model with chiral symmetry break- ing}

To reproduce the approximate $S U(2) \times S U(2)$ chiral symmetry of QCD, the hard-wall model of Refs. [69, 70] includes a higher-dimensional $S U(2) \times S U(2)$ gauge invariance. Treating isospin as unbroken, we consider the breaking of the chiral symmetry due to the chiral condensate $\sigma=\left\langle\left(\bar{u}_{L} u_{R}+\bar{u}_{R} u_{L}\right)\right\rangle=\left\langle\left(\bar{d}_{L} d_{R}+\bar{d}_{R} d_{L}\right)\right\rangle$. Correspondingly, the model includes a set of 5 -dimensional fields $X_{I i}, I, i \in(1,2)$, which transform as a bifundamental under the $S U(2) \times S U(2)$ gauge invariance. A non-vanishing background for $X_{I i}$ breaks the chiral symmetry via the Higgs mechanism. If we assume the breaking is such that

$$
\left\langle X^{I i}(x, z)\right\rangle=X_{0}(z) \delta^{I i}
$$

then the diagonal subgroup of the gauge group is unbroken by the background. This unbroken group is identified with isospin, and Kaluza-Klein modes of the corresponding gauge fields are identified with the rho meson and its radial excitations. The broken sector describes the axial-vector mesons and pions. The AdS/CFT correspondence motivates an identification of quark mass and chiral condensate as coefficients in the background $X_{0}(z)$. If no other sources of symmetry breaking are introduced in the model, this pattern of symmetry breaking ensures that approximate relations like the Gell-Mann-Oakes-Renner relation [336], which are due to the pattern of chiral symmetry breaking, are satisfied in the model [69]. However, it is important to ensure that the boundary conditions on fluctuations about the background are consistent with the pattern of chiral symmetry breaking [337].

The symmetry-breaking background solves the equations of motion for the fields $X^{I i}$ in the background space-time. For simplicity we assume that the scalar fields have a mass $m^{2}$ but otherwise no potential, though nontrivial potentials have been considered (for example, Ref. [222]). We further assume that there is little backreaction from the profile $X_{0}(z)$ on the space-time geometry. The action describing non-gravitational fluctuations 
in the model is $[69,70]$ :

$$
S=\int d^{5} x \sqrt{g} \operatorname{Tr}\left\{|D X|^{2}-m^{2}|X|^{2}-\frac{1}{2 g_{5}^{2}}\left(F_{L}^{2}+F_{R}^{2}\right)\right\}
$$

where $D_{\mu} X=\partial_{\mu} X-i A_{L \mu} X+i X A_{R \mu}, A_{L, R}=A_{L, R}^{a} T^{a}$, and $F_{L, R}^{\mu \nu}=\partial^{\mu} A_{L, R}^{\nu}-i\left[A_{L, R}^{\mu}, A_{L, R}^{\nu}\right]$, and indices are contracted with the AdS metric of Eq. (7.1).

The scalar field equations of motion for the background $X_{0}(z)$ are:

$$
\frac{d}{d z}\left(\frac{1}{z^{3}} \frac{d}{d z} X_{0}\right)+\frac{m^{2} R^{2}}{z^{5}} X_{0}=0
$$

with solutions

$$
X_{0}(z)=m_{q} z^{4-\Delta}+\frac{\sigma}{4(\Delta-2)} z^{\Delta},
$$

where we assume $2<\Delta<4$ and we take $m_{q}$ and $\sigma$ real.

According to the AdS/CFT dictionary, the mass $m$ of a 5-dimensional $p$-form field depends on the scaling dimension $\Delta$ of the corresponding operator, according to (I.22)

$$
m^{2}=(\Delta-p)(\Delta+p-4) .
$$

This relation also follows from demanding the correct scaling behavior of correlation functions deduced by the AdS/CFT correspondence in the conformal field theory [27, 28], or in the deep Euclidean regime in models of asymptotically free confining theories. For example, for a conserved current, $\Delta=3$ and $p=1$, corresponding to a massless 5dimensional gauge field as discussed above. In AdS/QCD models it is reasonable to leave the effective scaling dimension $\Delta$ of most operators as adjustable parameters, because renormalization modifies the scaling dimension of operators at low energies. However, conserved currents are not renormalized and always correspond to massless 5-dimensional gauge fields in the dual theory.

For scalar fields like $X_{I i}, p=0$ and the exponent $\Delta$ is related to the mass $m$ via,

$$
m^{2}=\Delta(\Delta-4)
$$

A QCD operator with appropriate quantum numbers to be dual to $X_{I i}$ is the scalar quark bilinear $\left\langle\bar{q}_{L}^{I} q_{R}^{i}\right\rangle$ where $\left(q^{1}, q^{2}\right)=(u, d)$, which in the UV has dimension $\Delta=3$.

In the bottom-up approach we treat the 5-dimensional scalar mass squared, $\mathrm{m}^{2}$, as 
a free parameter ${ }^{1}$. However, for definiteness we can fix $m^{2}=-3 / R^{2}$, so that $\Delta=3$. The action Eq. (7.34) with $m^{2}=-3 / R^{2}$, together with the background space-time geometry Eq. (7.1) between $z=0$ and $z_{m}$, defines one version of the hard-wall AdS/QCD model $[69,70]$.

The solution $X_{0}(z) \propto z^{4-\Delta}$ is not normalizable in the sense defined earlier: For scalar field profiles of the form $X_{I i}(x, z)=X_{0}(z) X_{I i}(x)$, the integral over $z$ in the action is divergent if $X_{0}(z) \propto z^{4-\Delta}$ if $\Delta>2$. On the other hand, the solution $X_{0}(z) \propto z^{\Delta}$ is normalizable. The coefficients of the two solutions are defined in anticipation of their physical interpretation via the AdS/CFT correspondence. The normalizable solution to the equation of motion corresponds to the state of the system [338, 339]. In this case the vacuum expectation value of the operator corresponding to the field $X$, which is proportional to the chiral condensate $\sigma$ (We assume that $\sigma$ in Eq. (7.36) is real). The nonnormalizable solution corresponds to the source for the corresponding operator, in this case the (isospin-preserving) quark mass [27, 28]. The factor of $1 / 4(\Delta-2)$ in Eq. (7.36) is suggested by the AdS/CFT correspondence [339, 113]. As noted by Cherman, Cohen and Werbos [340], the interpretation of $m_{q}$ and $\sigma$ with quark mass and chiral condensate is only up to a rescaling of $m_{q}$ and $\sigma$. Matching the holographic prediction of the two-point correlator of scalar operators $\bar{q} q$ with the QCD prediction at large Euclidean momentum, the parameter $m_{q}$ should be rescaled by $\sqrt{N_{C}} / 2 \pi$ and $\sigma$ by $2 \pi / \sqrt{N_{C}}$ in order for $m_{q}$ and $\sigma$ to represent the quark mass and chiral condensate, respectively [340]. Although the identification of parameters in the scalar field background with QCD parameters would follow from the AdS/CFT correspondence, they may more conservatively be regarded as model parameters allowed to vary in order to fit experimental data.

We define the vector and axial-vector combinations of the gauge field $V_{M}^{a}=\left(L_{M}^{a}+\right.$ $\left.R_{M}^{a}\right) / \sqrt{2}$ and $A_{M}^{a}=\left(L_{M}^{a}-R_{M}^{a}\right) / \sqrt{2}$, respectively. Under parity the gauge fields transform as $L_{i}^{a}(t, \mathbf{x}, z) \leftrightarrow-R_{i}^{a}(t,-\mathbf{x}, z), L_{0}^{a}(t, \mathbf{x}, z) \leftrightarrow R_{0}^{a}(t,-\mathbf{x}, z), L_{z}^{a}(t, \mathbf{x}, z) \leftrightarrow R_{z}^{a}(t,-\mathbf{x}, z)$. The vector combination $V_{i}^{a}=\left(L_{i}^{a}+R_{i}^{a}\right) / \sqrt{2}$ is odd under parity; the axial-vector combination $A_{i}^{a}=\left(L_{i}^{a}-R_{i}^{a}\right) / \sqrt{2}$ is even. Expanding the scalar fields about the background, we may write

$$
X(x, z)=\left(X_{0}(z)+\sigma(x, z)\right) \exp \left[2 i T^{a} \pi^{a}(x, z)\right],
$$

where $T^{a}$ are $S U(2)$ generators normalized so that $\operatorname{Tr} T^{a} T^{b}=\delta^{a b} / 2$. In this matrix notation, we also define $L_{M}=L_{M}^{a} T^{a}$ and $R_{M}=R_{M}^{a} T^{a}$, and similarly for $V_{M}$ and $A_{M}$. The Hermitian matrix of fields $\sigma(x, z)$ are scalar under Lorentz invariance and

\footnotetext{
${ }^{1}$ In the light-front holographic approach the 5 -dimensional mass $m$ is not free parameter, but it is fixed by the holographic mapping to the light-front (See Chapters 4 and 5).
} 
parity, and for the time being we set these fields to zero. The equations of motion for $\sigma, \pi$ and the gauge fields, with appropriate boundary conditions [337], determine the spectrum of Kaluza-Klein modes, which are identified with the corresponding meson states. The quantum numbers of the mesons are determined by transformations of the 5-dimensional fields under symmetries of the 5-dimensional theory. The gauge fields are odd under charge conjugation. Under 5-dimensional parity the vector $V_{\mu}^{a}$ is odd; the axial-vector $A_{\mu}^{a}$ is even. Hence, the Kaluza-Klein modes of the transverse part of $V_{\mu}^{a}$ are identified with the tower of radial excitations of rho mesons; the Kaluza-Klein modes of the transverse part of $A_{\mu}^{a}$ are identified with the tower of radial excitations of $a_{1}$ mesons. The fields $\pi^{a}$, which mix with the longitudinal part of $A_{\mu}^{a}$, are odd under charge conjugation and parity. The solutions to the coupled equations of motion for $\pi^{a}$ and the longitudinal part of $A_{\mu}^{a}$, with appropriate boundary conditions, are identified with the pions.

The decay constants for the vector and axial-vector mesons may be calculated either from the AdS/CFT correspondence as described above, or by examination of the effective action for the Kaluza-Klein modes upon integration of the action over the $z$-coordinate [38]. The model defined above has three free parameters: $m_{q}, \sigma$, and $z_{m}$. A global fit for the lightest pion, rho and $a_{1}$ masses and decay constants yields agreement with data at better than $10 \%$ [69]. The effective action also determines couplings like $\rho-\pi-\pi$, which generally do not fare as well. However, the existence of a non-vanishing $\rho-\pi-\pi$ coupling in the model predicts a non-vanishing width for the rho meson, which would then shift the corresponding pole of the vector current two-point correlator off the real axis.

The addition of the strange quark is straightforward. The $S U(2) \times S U(2)$ gauge group becomes $S U(3) \times S U(3)$, and to separate the strange quark mass from the up and down quark masses one can expand about the background $X_{0}(z)=\operatorname{diag}\left(m_{q}, m_{q}, m_{s}\right) z+$ $\sigma z^{3} / 4$, as in Ref. [341]. The result of a five-parameter model to fourteen observables is given in Table 7.1 from Ref. [341].

\subsection{Top-down models}

Top-down AdS/QCD models are based on configurations of D-branes in string theory are more restrictive than bottom-up models. Strings ending on D-branes have a fieldtheoretic interpretation at low energies, and the AdS/CFT correspondence provides a dual description of the field theory in terms of a supergravity or string theory in a fixed background space-time with fluxes of certain fields. The class of QCD-like theories which 


\begin{tabular}{|c|c|c|}
\hline Observable & $\begin{array}{c}\text { Measured } \\
\text { (Central Value-MeV) }\end{array}$ & $\begin{array}{c}\text { Model Fit } \\
(\mathrm{MeV})\end{array}$ \\
\hline$m_{\pi}$ & 139.6 & 134 \\
$f_{\pi}$ & 92.4 & 86.6 \\
$m_{K}$ & 496 & 514 \\
$f_{K}$ & 113 & 101 \\
$m_{K_{0}^{*}}$ & 672 & 697 \\
$f_{K_{0}^{*}}$ & & 36 \\
$m_{\rho}$ & 776 & 789 \\
$F_{\rho}^{1 / 2}$ & 345 & 335 \\
$m_{K^{*}}$ & 894 & 821 \\
$F_{K^{*}}$ & & 337 \\
$m_{a_{1}}$ & 1230 & 1270 \\
$F_{a_{1}}^{1 / 2}$ & 433 & 453 \\
$m_{K_{1}}$ & 1272 & 1402 \\
$F_{K_{1}^{1 / 2}}$ & & 488 \\
\hline
\end{tabular}

Table 7.1: Fit of a five-parameter hard-wall model for meson masses and decay constants, from Ref. [341].

emerge from D-brane configurations with supergravity duals is rather limited, and those theories each differs from QCD in a number of important ways. Top-down models are motivated rather directly by the AdS/CFT correspondence, so this is an appropriate point to briefly review that motivation.

In the top-down approach, a brane configuration in string theory is engineered whose low-energy spectrum of open-string fluctuations has a known field-theoretic interpretation. Via the AdS/CFT correspondence, for some brane constructions describing large$N_{C}$ gauge theories with large 't Hooft coupling $g^{2} N_{C}$, a dual description exists in terms of supergravity on a fixed space-time background supported by fluxes of certain fields [22]. The prototypical example is $\mathcal{N}=4$ supersymmetric gauge theory, which has as its dual (in the supergravity limit $N_{C} \rightarrow \infty$ and $g^{2} N_{C} \gg 1$ ) Type IIB supergravity on an $\mathrm{AdS}_{5} \times S^{5}$ space-time background with non-vanishing five-form flux and constant dilaton.

For this prototypical example Maldacena considered the dynamics of a stack of over- 
lapping D3-branes in Type IIB string theory [29]. The number 3 in "D3-branes" indicates that the branes span three spatial dimensions. Quantizing the open strings which end on the $N_{C}$ D3-branes, one finds the massless spectrum of $\mathcal{N}=4$ supersymmetric $S U\left(N_{C}\right)$ gauge theory in $3+1$ dimensions. The massless spectrum of the closed strings describes Type IIB supergravity, which includes a scalar dilaton field and a spin-2 graviton, in addition to various $p$-form and fermionic fields. The gauge coupling $g$ in the action for the $S U\left(N_{C}\right)$ gauge theory on the D3-branes is also related to the string coupling $g_{s}$, by $g_{s}=g^{2}$.

If $g_{s} \rightarrow 0$ then perturbative string theory corrections are negligible, and classical supergravity is an appropriate description of the theory. In addition to a non-vanishing constant dilaton, the solutions to the supergravity equations of motion include a nonvanishing five-form flux (for which the D3-branes act as sources) and a space-time with horizon. The near-horizon geometry of this space-time is the aforementioned $\operatorname{AdS}_{5} \times S^{5}$. The curvature of both the anti-de Sitter factor and the five-sphere is given by a distance scale $R_{5}=l_{s}\left(g_{s} N_{C}\right)^{1 / 4}$, where $l_{s}$ is the string length. Hence, if $g_{s} N_{C} \gg 1$ then the geometries are smooth on scales of the string length, and massive string modes can be neglected so that fluctuations are described by classical supergravity without higherderivative operators induced by the massive modes. In this case the 't Hooft coupling $g^{2} N$ is large in the gauge theory on the D3-branes. The supergravity limit, in which classical supergravity describes the fluctuations of the background created by the stack of D-branes, is what we have just described: $g_{s} \rightarrow 0$ with $g_{s} N_{C} \gg 1$, or equivalently, $N_{C} \rightarrow \infty$ with $g^{2} N_{C} \gg 1$.

Maldacena's great conceptual leap was to identify the physics of the open strings (i.e. $\mathcal{N}=4$ Yang-Mills theory) with the physics of the closed strings in the nearhorizon geometry (i.e. $\operatorname{AdS}_{5} \times S^{5}$ ). Some evidence for the duality is provided by a matching of symmetries, in particular the $S O(2,4)$ conformal symmetry and the $S O(6)$ $R$-symmetry of the $\mathcal{N}=4$ Yang-Mills theory, which are identical to the isometries of $A d S_{5}$ and $S^{5}$, respectively. Certain classes of operators in the $\mathcal{N}=4$ theory naturally map onto the spherical harmonics on the $S^{5}$. The explicit dictionary between the dual theories was proposed independently by Witten [28] and Gubser, Klebanov and Polyakov [27]. Operators in the field theory correspond to supergravity fields on $\mathrm{AdS}_{5}$. In the supergravity limit $N_{C} \rightarrow \infty$ with $g^{2} N_{C} \gg 1$, the generating functional for connected correlation functions in the $\mathcal{N}=4$ theory is identified with the action in the supergravity on the $\mathrm{AdS}_{5} \times S^{5}$ background, with the constraint that the supergravity fields approach $(3+1)$-dimensional configurations on the boundary of $\mathrm{AdS}_{5}$ that are the sources for the 
corresponding operators in the $\mathcal{N}=4$ theory.

Karch and Katz [328] suggested the possibility to add to the basic scenario a small number of matter fields that transform in the fundamental representation of the $S U\left(N_{C}\right)$ gauge group by adding to the $N$ D3-branes a small number of D7-branes. The light fluctuations of strings which stretch from the D7-branes to the D3-branes include scalar fields and fermions which transform in the fundamental representation of the $S U\left(N_{C}\right)$ gauge group, in analogy to the quarks of $S U(3)$ QCD. The resulting theory is not asymptotically free, but progress is made by treating the D7-branes as probes while ignoring their backreaction on the supergravity background, in which case the difficulties associated with the loss of asymptotic freedom are conveniently evaded.

There now exists a large number of examples of field theories with supergravity duals, and the basic dictionary has been expanded to include theories with interesting phenomenological features. Conformal invariance and supersymmetry are not essential. The field theory can be confining with chiral symmetry breaking, which in those respects is similar to QCD. Some examples of confining theories with known supergravity duals are the $\mathcal{N}=1^{*}$ theory of Polchinski and Strassler [32], the Klebanov-Strassler cascading gauge theory [33], the D4-D6 system of Kruczenski et al. [53], and the D4-D8 system of Sakai and Sugimoto [38, 39]. The predictions of a top-down model pertain to the specific theory dual to the particular supergravity background defining the model. The benefit of top-down models is that both sides of the duality can be described, at least in part, independently of the duality. Top-down AdS/QCD models are engineered to have certain similarities to QCD, but always suffer from a difficulty in separating scales of the desired degrees of freedom from additional degrees of freedom and interactions not present in QCD.

Although the AdS/CFT correspondence is conjectured to extend to smaller $N_{C}$ by extending the supergravity to the full string theory, for the sake of calculability AdS/QCD models generally ignore the string-theoretic corrections and naively extend the supergravity description of the AdS/CFT correspondence to QCD, with $N_{C}=3$. Certain remnants of the large- $N_{C}$ approximation remain at first approximation in both top-down and bottom-up AdS/QCD models, such as infinitely narrow resonances. Additional difficulties in matching aspects of QCD, such as the distribution of jets at high energies, have been discussed by various authors, for example [115, 342]. 

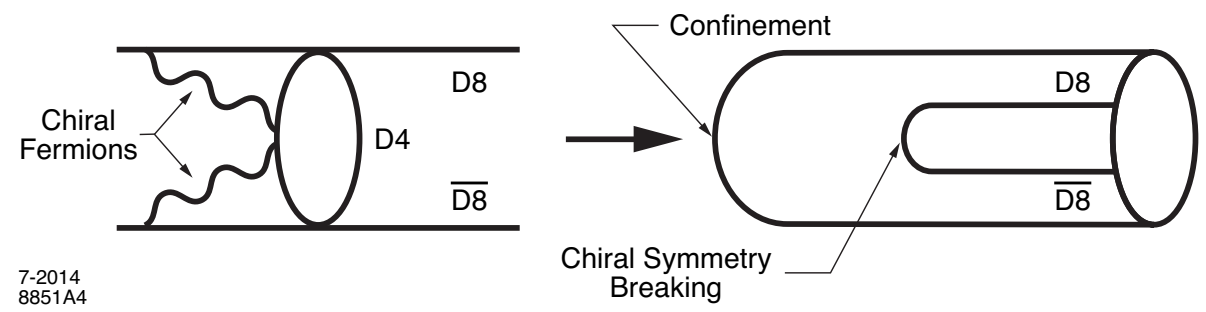

Figure 7.1: Brane configuration in the Sakai-Sugimoto model. The D4-branes create a cigarlike geometry, and the D8 and $\overline{\mathrm{D} 8}$-branes connect. A mass gap is created by the cigar-like box, and chiral symmetry breaking arises from the joining of the branes responsible for the left and right-handed chiral fermions.

\subsection{The Sakai-Sugimoto model}

The Sakai-Sugimoto model is so far the top-down application of the AdS/CFT correspondence most closely related to QCD. The system includes a stack of $N$ D4-branes in Type IIA string theory [29] wrapped on a circle on which fermions satisfy antiperiodic boundary conditions which break the supersymmetry of the theory. From an effective $3+1$ dimensional point of view, the massless spectrum includes the $S U(N)$ gauge fields, but the fermions and scalars are massive. At low energies, the theory is described by pure Yang-Mills theory. Without the additional flavor branes introduced by Sakai and Sugimoto, this model was discussed by Witten shortly after the initial AdS/CFT conjecture [34] in the context of the deconfinement transition in QCD. In that case the circle is the compactified Euclidean time, whose size is inversely related to the temperature of the system. Kruczenski et al. [53] suggested the addition of probe D6 and anti-D6 $(\overline{\mathrm{D} 6})$ branes to the model, which gives rise to fermionic quark fields in the low-energy spectrum, but without the pattern of chiral symmetry breaking observed in nature. Instead of the probe D6-branes, the Sakai-Sugimoto model includes $N_{f}$ D8-branes and $N_{f}$ $\overline{\mathrm{D}} 8$-branes transverse to the circle on which the D4-branes wrap. The D8 and $\overline{\mathrm{D} 8}$ branes span all of the nine spatial dimensions of the string theory except the circle on which the D4-branes are wrapped, and they intersect the D4-branes on 3+1 dimensional manifolds at definite positions along the circle, as in Fig. 7.1. The massless fluctuations of open strings connecting the D4 and D8 or D8-branes at their intersections describe $3+1$ dimensional chiral fermions, with opposite chirality at the D8 and $\overline{\mathrm{D}} 8$-branes. This is the Sakai-Sugimoto model $[38,39]$. There are two "ultraviolet" regions on the D8-branes, for which the geometry is asymptotically different than AdS, and the gauge theory dual to the Sakai-Sugimoto model is not asymptotically free. In fact, since the geometry 
in this model is not asymptotically AdS, it cannot account for dimensional power scaling $[55,56,57,58]$ in QCD for hard scattering. The Sakai-Sugimoto model should be considered a model of QCD only at low energies.

In the supergravity limit the D4-branes generate a space-time with a cigar-like topology which effectively cuts off the space-time geometry at the tip of the cigar. The $9+1$ dimensional space-time metric generated by the D4-branes wrapped on a circle is [34]

$$
d s^{2}=\left(\frac{U}{R}\right)^{3 / 2}\left(\eta_{\mu \nu} d x^{\mu} d x^{\nu}+f(U) d \tau^{2}\right)+\left(\frac{R}{U}\right)^{3 / 2}\left(\frac{d U}{f(U)}+U^{2} d \Omega_{4}^{2}\right)
$$

where the Greek indices span $3+1$ dimensions, $\tau$ is the coordinate on the circle, and $d \Omega_{4}^{2}$ is the metric on the unit four-sphere. The coordinate $U$ plays a role analogous to the radial coordinate $z$ of Anti-de Sitter space in the holographic interpretation of this model. The function $f(U)$ describes the horizon at some $U=U_{\mathrm{KK}}$ :

$$
f(U)=1-\frac{U_{\mathrm{KK}}^{3}}{U^{3}}
$$

As $U$ approaches the horizon at $U_{K K}$ the proper size of the circle in the $\tau$-direction shrinks to zero size. For the geometry to be smooth, the location of the tip of the cigar is correlated with the size of the compact circle on which the D4-branes wrap [34, 53]. This is one reason that non-QCD states are not decoupled from the hadronic states of interest. The dilaton $\phi$ also has a profile in the D4-brane background, and plays a role in the action on the D8-branes:

$$
e^{\phi}=g_{s}\left(\frac{U}{R}\right)^{3 / 4}
$$

where $g_{s}$ is the string coupling. The D4-branes also generate a 4-form flux which supports the space-time geometry, but otherwise that flux does not play a role in the present discussion.

In the D4-brane background, ignoring the backreaction of the D8 and $\overline{\mathrm{D} 8}$-branes on the geometry, the D8 and $\overline{\mathrm{D} 8}$-branes bend as a consequence of their tension so as to minimize the Dirac-Born-Infeld action on the branes. The D8-brane profile is described by a curve in the $U-\tau$ coordinates, which is specified by a function $U=U(\tau)$. The result is that the $\mathrm{D} 8$ and $\overline{\mathrm{D}} 8$-branes connect at some minimum value of $U$, which corresponds to the spontaneous breaking of the $S U\left(N_{f}\right) \times S U\left(N_{f}\right)$ chiral symmetry of the theory to a diagonal $S U\left(N_{f}\right)$ isospin symmetry. 
The perturbative massless spectrum is that of $\mathrm{SU}(N)$ QCD with $N_{f}$ flavors of quarks. However, Kaluza-Klein modes associated with the circle direction have masses comparable to the confining scale in this theory, so the massive spectrum of QCD-like bound states are not separated in mass from the spectrum of non-QCD-like KaluzaKlein modes. As a result, the five-dimensional nature of the effective theory on the D4-branes becomes apparent at the same scale as the hadron masses we are interested in. This is an important distinction between the Sakai-Sugimoto model and QCD, and typically the unwanted states are ignored when comparing the model to experiment.

If we ignore the Kaluza-Klein modes around the circle, and integrate out the four dimensions along the D8-branes but transverse to the D4-branes, then the fluctuations of the $4+1$ dimensional $S U\left(N_{f}\right) \times S U\left(N_{f}\right)$ gauge fields on the $\mathrm{D} 8$ and $\overline{\mathrm{D} 8}$-branes are identified with vector mesons, axial-vector mesons, and pions. The quantum numbers of the corresponding states can be identified with symmetries of the D-brane system. The $3+1$ dimensional parity symmetry, for example, is identified with a parity symmetry in the $4+1$ dimensional theory, which also exchanges the two sets of $S U\left(N_{f}\right)$ gauge fields. If we ignore the extra circle direction, then the effective $3+1$ dimensional action on the D8-branes at the intersection with the D4-branes describes the effective action for the light mesons, and allows for the comparison of decay constants $\left(f_{\pi}, F_{\rho}\right.$, etc. $)$ and couplings (e.g. $g_{\rho \pi \pi}$ ), or alternatively some of the chiral low-energy coefficients, with QCD. At this stage the setup is similar to that of bottom-up models, except that the D8-branes have two asymptotic UV boundaries (one for each $S U\left(N_{f}\right)$ factor), and the $\mathrm{AdS}_{5}$ space-time is replaced by the induced metric on the D8-branes,

$$
d s^{2}=\left(\frac{U}{R}\right)^{3 / 2} \eta_{\mu \nu} d x^{\mu} d x^{\nu}-\left(\frac{R}{U}\right)^{3 / 2}\left[\frac{1}{f(U)}+\left(\frac{U}{R}\right)^{3} \frac{f(U)}{U^{\prime}(\tau)^{2}}\right] d U^{2}-\left(\frac{R}{U}\right)^{3 / 2} U^{2} d \Omega_{4}^{2}
$$

and the four-sphere is integrated over in the effective 4+1-dimensional action, after which it no longer plays an important role. (This is analogous to the role of the five-sphere in the prototypical $\mathrm{AdS}_{5} \times S^{5}$ geometry in the $\mathrm{AdS} / \mathrm{CFT}$ correspondence.)

Once the effective $4+1$ dimensional action for the gauge fields on the D8-brane is evaluated, the calculation of meson observables proceeds as in bottom-up models. One difference of note is that there are two UV boundaries in the Sakai-Sugimoto model, one for each factor of $S U\left(N_{f}\right)$ in the chiral symmetry. There is one set of $S U\left(N_{f}\right)$ gauge fields in the geometry with two ultraviolet regions rather than two sets of $S U\left(N_{f}\right)$ gauge fields in the hard-wall model, with one ultraviolet region. The potential to enhance 
the symmetries of a theory by way of additional ultraviolet regions was exploited in unrelated models in Ref. [343].

Several related descriptions of light baryons in AdS/QCD have been identified. The effective 4+1 dimensional gauge theory on the D8-branes, after integrating out the additional 4-sphere in the D8-brane geometry, has small instanton solutions which correspond to D4-branes wrapped on the 4-sphere [190, 189]. Also integrating out the remaining extra dimension along the induced geometry on the D8-branes, the Skyrme term in the effective $3+1$ dimensional pion Lagrangian emerges directly from the kinetic terms in the action for the $S U(2)_{L} \times S U(2)_{R}$ gauge fields [38, 39]. The baryon number of the Skyrmion is equivalent to the instanton number in the $4+1$ dimensional effective theory [190], so the Sakai-Sugimoto model provides a connection between various solitonic descriptions of the baryon [344]. A similar discussion in a bottom-up model was given in Ref. [345].

Despite the differences between the Sakai-Sugimoto model and QCD, the qualitative, and sometimes quantitative, success of a naive application of the AdS/CFT correspondence when compared with QCD has helped to further the hope that interesting features of QCD might be better understood by consideration of holographic models. The geometry on which the D8-branes live is similar to one considered earlier by Son and Stephanov, albeit in latticized form, in the context of extended hidden local symmetry models [346]. The Sakai-Sugimoto model is also similar to the hard-wall model with chiral symmetry breaking, although the quarks in the Sakai-Sugimoto model are exactly massless. An alternative approach to chiral symmetry breaking with massive quarks in a system with D7 branes in a nontrivial background was suggested in Ref. [347]. 


\section{Chapter 8}

\section{Summary and Conclusion}

The AdS/CFT correspondence (or gauge/gravity duality) introduced by Maldacena [22] has given rise to a completely new set of tools for studying the dynamics of strongly coupled quantum field theories such as QCD. In effect, the strong interactions of quarks and gluons are represented by a simpler classical gravity theory in a higher-dimensional space. Anti-de Sitter space in five dimensions plays a special role in elementary particle physics because it provides an exact geometrical representation of the conformal group.

Although a perfect string theory dual of QCD is not yet known, bottom-up approaches, starting from a QCD description and searching for a higher-dimensional theory, of which QCD is the boundary theory, has already provided many new and remarkable insights into QCD. In particular one has to break by some mechanism the maximal symmetry of AdS space, because its holographic dual quantum field theory is conformally invariant and cannot incorporate a mass scale like $\Lambda_{\mathrm{QCD}}$.

In this report we mainly concentrate on light-front holographic QCD [74, 75, 91]. This approach to strongly coupled quantum field theory exploits the remarkable holographic duality between classical gravity in $\mathrm{AdS}_{5}$ and the semiclassical approximation to light-front quantized QCD. Light-front Hamiltonian theory, derived from the quantization of the QCD Lagrangian at fixed light-front time $x^{+}=x^{0}+x^{3}$, provides an ideal framework for describing bound-states in relativistic theory (Chapter 2). Light-front holography leads to a precise relation between the holographic variable $z$ of the fifth dimension of AdS space, and the light-front variable $\zeta$, the argument of the boost-invariant light-front wave functions describing the internal structure of hadrons in physical spacetime [75].

For massless quarks the classical QCD Lagrangian contains no scale and is con- 
formal invariant. However, the appearance of a scale, or a mass gap, is necessary for confinement. Therefore the origin of a mass scale in an originally conformal theory is a fundamental unsolved problem in the theory of strong interactions. An indication of the origin of the QCD mass scale can be drawn from a remarkable paper by V. de Alfaro et. al. [94] in the context of one-dimensional quantum field theory. Starting from a conformally invariant action these authors showed how to construct a generalized Hamiltonian from the the three generators of the conformal group in one dimension. This remarkable result is based in the fact that the $S O(2,1)$ algebra can be realized in conformal quantum mechanics: one of the generators of $S O(2,1)$, the rotation in the 2-dimensional space, is compact and has therefore a discrete spectrum. Mathematically, this result is based on the isomorphism of the one-dimensional conformal group $\operatorname{Conf}\left(R^{1}\right)$ with the group $S O(2,1)$. Since the generators of the conformal group $H, D$ and $K$ have different dimensions their connection with the generators of $S O(2,1)$ require the introduction of a scale [94], which plays a prominent role in the dAFF procedure. The evolution parameter corresponding to the $\mathrm{dAFF}$ generalized Hamiltonian is proportional to the light front-time and has a finite range [95].

The threefold connection of light-front dynamics, classical gravity in a higher-dimensional space, and a conformal invariant one-dimensional quantum field theory provides new insights into the origin of a fundamental mass scale and the physics underlying confinement dynamics in QCD [95]. The mapping of the generalized dAFF Hamiltonian to the light front fixes the effective instantaneous light-front potential to a harmonic oscillator form (See Chapter 3). This corresponds to a quadratic dilaton profile in the embedding AdS space. For large separation distances the quadratic effective potential in the front form of dynamics corresponds to a linear potential in the usual instant form of dynamics [97]. The final result is a relativistic light-front wave equation for arbitrary spin which incorporates essential spectroscopic and dynamical features of hadron physics.

The light-front Hamiltonian equation predicts that the pion has zero mass for massless quarks, and the resulting Regge trajectories have equal constant slope in the radial and orbital quantum numbers as observed experimentally. There is only one input, the constant $\sqrt{\lambda}$ setting the QCD mass scale (See Chapter 5). Our procedure can be extended to non-zero light quarks without modifying to first approximation the transverse dynamics and the universality of the Regge slopes (See Sec. 5.1.3). The predicted meson light-front wave function accurately describes diffractive vector meson electroproduction [246] and other observables. The shape of form factors is successfully described (See Chapter 6). 
We have derived hadronic bound-state equations for hadrons with arbitrary spin starting from an effective invariant action in the higher-dimensional classical gravitational theory. The mapping of the equations of motion from the gravitational theory to the Hamiltonian equations in light-front quantized QCD has been an important guide to construct the effective actions in the bulk and to separate kinematical and dynamical aspects [91]. For mesons, this separation determines a $J$-dependent constant term in the effective potential. The unmodified AdS geometry reproduces the kinematical aspects of the light-front Hamiltonian, notably the emergence of the light-front angular momentum which is holographically related to the AdS mass in the gravitational theory, and the modification of AdS space in the infrared region encodes the dynamics, including confinement (See Chapter 4).

For baryons the 3-body state is described by an effective two-body light-front Hamiltonian, where the holographic variable is mapped to the invariant separation of one constituent (the active constituent) to the cluster of the rest (the spectators). Therefore, the mapping of AdS equations to the light-front bound-state equations imply that there is only one relevant angular momentum, the light-front orbital angular momentum $L$ between the active and the spectator cluster, an effective approximation which captures much of the strongly-coupled dynamics. Furthermore, since the action for fermions is linear in the covariant derivatives, no mixing between dynamical and kinematical aspects occurs. Thus, for baryons there is no explicit $J$ dependence in the light-front equations of motion, and consequently the bound-state spectrum of baryons can only depend on $L$ (See Sec. 5.2.1). These remarkable predictions, which are inferred from the geometry of AdS space, are independent of the specific dynamics and account for many striking similarities and differences observed in the systematics of the meson and baryon spectra [91]. The equality of the slopes of the linear Regge trajectories and the multiplicity of states for mesons and baryons is explained. We also explain the observed differences in the meson versus the baryon spectra that are due to spin-orbit coupling. For example, the predicted triplet spin-orbit splitting for vector mesons is in striking contrast with the empirical near-degeneracy of baryon states of different total angular momentum $J$; the baryons are classified by the internal orbital angular momentum quantum number $L$ along a given Regge trajecory, not $J$ (See Chapter 5).

The semiclassical approximation described here is not restricted to a specific number of colors. Indeed, in this effective theory the color quantum number does not appear explicitly. However, since the model is an offspring of the original AdS/CFT correspondence [22], it is reminiscent of an $N_{C} \rightarrow \infty$ theory. This interpretation is also in 
accordance with the zero width of all states, including the excited ones.

The treatment of the chiral limit in the LF holographic approach to strongly coupled QCD is substantially different from the standard approach. In the standard approach spontaneous chiral symmetry breaking plays the crucial role. The massless pion is the Goldstone boson of the broken symmetry and the mass differences between the parity doublets, for example the $\rho(770)-a_{1}(1260)$ and the $N(939)-N(1535)$ doublets, is a consequence of the spontaneous chiral symmetry breaking. In light-front holographic QCD discussed here, the vanishing of the pion mass in the chiral limit follows from the precise cancellation of the light-front kinetic energy and light-front potential energy terms for the quadratic confinement potential (See Sec. 5.1.1). This effective potential results from the triple correspondence of light-front quantized QCD, gravity in $\mathrm{AdS}_{5}$ space and conformal quantum mechanics [95]. The mass differences between the parity doublets also follows from this specific potential. The parity splitting in this framework depends crucially on the light-front orbital quantum numbers. Therefore, in this approach the parity doublets are not degenerate and the trajectories remain parallel as observed experimentally (See Chapter 5).

The mapping of transition amplitudes in the gravity theory to the light front is also an important aspect of light-front holography (See Chapter 6). In addition to reproducing the essential elements of the transverse dynamics found by the light-front mapping of the Hamiltonian equations, one also obtains new information on the longitudinal dynamics which is relevant, for example, to compute QCD distribution amplitudes and extend the formalism to include light-quark masses (See Sec. 5.1.3).

In addition to describing hadronic bound states (normalizable solutions), the gravity theory allows to extend the confining dynamics to external currents (non-normalizable solutions). The "dressed" or confined current corresponds to sum an infinite class of Fock states containing $q \bar{q}$ pairs in the hadronized current. This leads to the remarkable results that for the soft-wall model the current is expressed as an infinite sum of poles and the form factor as a product of poles (See Sec. 6.1.4). Notably, the actual number of poles in form factor is determined by the twist of the hadronic state (the number of constituents). At large space- and time-like $q^{2}$ the form factor incorporates the correct power-law fall-off for hard scattering independent of the specific dynamics and is dictated by the twist [55]. At low $q^{2}$ the form factor leads to vector dominance, and therefore there are no divergences in the limit of zero quark masses. Furthermore the analytic expressions obtained for the form factors allows an analytic continuation into the timelike region (See Sec. 6.1.5). 
Finally, in Chapter 7 we reviewed bottom-up and top-down holographic models motivated by chiral symmetry breaking in QCD. We described in this chapter the most relevant aspects of this approach and practical limitations. Hopefully future studies will help to understand better the connections between both approaches.

\subsection{Open problems and future applications}

The light-front holographic model discussed here gives a satisfactory first-order description of a large bulk of light-hadronic data using essentially a single scale. It is of course desirable to improve the agreement with observations and to better understand some aspects of the framework. We collect the most noticeable points that from our perspective should be addressed.

1. The agreement for the meson trajectories of the $\rho$ and $K^{*}$ is very satisfactory. For the mesons on the $\pi$ and $K$ trajectory the agreement is less satisfactory and the scale $\sqrt{\lambda}$ has to be increased by $10 \%$. The triplet splitting of the a mesons is qualitatively correct, but the predicted splitting is too large.

2. An extended computation should also include the isoscalar mesons, the description of which is problematic in most models [208]. A proper description should probably include higher twist components. For example, the $f_{0}$ could be a superposition of two quarks in a $P$ wave and four quarks in an $S$ wave.

3. In the soft-wall model the dilaton profile in the AdS action for fermions does not lead to an effective potential, since it can be absorbed by a field redefinition [182]. Thus confinement must be imposed by introducing an additional term in the action (See Sect. 4.3).

4. The agreement in the full baryon sector is also very satisfactory. Indeed, two newly discovered states [16], the $N(1875)$ and the $N(1900)$ are well described by the model. However, the quantum number assignment for the $\nu$ quantum number (See Sec. 5.2.1) is only fixed for the proton trajectory. For the other families the assignment is phenomenological. We expect that a further investigation of the light-front mapping to baryonic states with different quark configurations will explain this successful assignment.

5. The $\nu$-assignment of the proton mentioned above is based on the fact that it is the ground state. In our approach it corresponds to a bound state of an active quark 
and the remaining cluster. Therefore the number of effective constituents in the nonperturbative domain is 2 , corresponding to an effective twist 2 . In the nucleon this corresponds to a quark-diquark cluster decomposition. At short distances all constituents in the proton are resolved and therefore the fall-off of the form factor at high $q^{2}$ is governed by the number of all constituents, $i$. e., twist 3 . It is desirable to understand better the dynamics of the cluster formation in the nucleon and thus get further insight into the transition region of the nonperturbative to the perturbative regime.

6. The description of electromagnetic form factors in the soft-wall model is very satisfactory if the poles of the confining electromagnetic current $(J=1, L=1)$ are shifted to their physical locations (See Sec. 6.1.4), which corresponds to the predicted bound-state poles of the $\rho$ ground state and its radial excitations $(J=$ $1, L=0)$.

7. To extend the computation of form-factors in the time-like region for larger values of $q^{2}$ one has to include finite decay widths with a correct threshold behavior. The present analysis only included constant decay widths and Fock states up to twist four (See Sec. 6.1.5).

8. We have used successfully the $S U(6)$ spin-flavor symmetry only for the computation of the Dirac form factor. The computation of the Pauli form factor was carried out using the generic expression for a twist-4 hadronic state normalized to the experimental anomalous magnetic moment (See Sec. 6.2.1). In fact, the value of anomalous magnetic moment and the actual form of the Pauli form factor should result from the theory.

9. For particles with spin, the AdS wave function is factorized as product of a $z$ independent polarization tensor and a z-dependent scalar function (See Chapter 4). This implies that the light-front wave function is the same for all polarizations, which is not a realistic result. This does not affect the spectrum, since the mass eigenvalue is independent of the polarization. But for electroproduction of vector mesons, for instance, the different polarizations give different contributions, and therefore their precise form is important. A more elaborate model, for instance starting from a Duffin-Kummer-Petiau equations [348, 349, 350] in AdS, could solve this problem. 
10. An essential feature for the construction of a confining Hamiltonian from a conformal action is the transition from the original evolution parameter $t$ to a new evolution parameter $\tau$, which is proportional to the light-front lime $x^{+}$and has a finite range (See Chapter 3). It is natural to identify this $\tau$ as the LF time difference of the confined $q$ and $\bar{q}$ in the hadron, a quantity which is naturally of finite range and in principle could be measured in double-parton scattering processes.

11. The emergence of a confining light-front Hamiltonian here was obtained by rather formal arguments. One would like to relate this derivation to more conventional methods used in QCD like the Dyson-Schwinger approach or the summation of H diagrams (two-gluon exchange with gluonic multi-rungs) which are infrared divergent $[163,164]$.

We have discussed and successfully applied an extension of the light-front mapping to include light-quark masses in the Hamiltonian and the light-front wave functions (LFWFs). The formal procedure could be extended to heavy masses, but then conformal symmetry can no longer be a guiding principle and there is no reason that the harmonic oscillator light-front potential remains valid. Furthermore additional contributions as the one gluon-exchange potential become important. There are many applications in light-front QCD [351] that require knowledge of the AdS/light-front wave functions. We list a few of them:

a) The nucleon transition form factor to the first radial excitation using light-front holographic methods has been computed in Ref. [319]. With the $12 \mathrm{GeV}$ upgrade at Jefferson Lab, it will be possible to measure different nucleon form factors to higher excited states at high virtuality [352]. The methods of Ref. [319] can be extended to compute these quantities.

b) The shape of the pion light-front wave function is measured in diffractive dijet reactions $\pi A \rightarrow$ Jet Jet $X$ [353]. The data shows a Gaussian fall-off in $k_{\perp}$ and a transition at high $k_{\perp}$ to power-law fall-off. This could be a testing ground of LF holography and the confinement potential. Using the holographic LFWFs, we can predict, for example, the slope in $k_{\perp}^{2}$ and the change in the shape in $k_{\perp}$ with $x$.

c) Using the light-front holographic wave functions obtained here one can in principle compute hadronization at the amplitude level [354]. 
d) The holographic light-front wave functions can be used to compute the quark interchange contributions to exclusive hadronic amplitudes [292].

e) Since the transverse holographic light-front wave functions form a complete basis, they can be used as a starting point to compute higher order corrections, as for example using the Lippmann-Schwinger equation or the coupled-cluster method [355]. The light-front wave functions also provide a convenient basis for numerical computations as in the BFLQ approach [356].

There are two conceptually essential points which need further clarification. First, the existence of a weakly-coupled classical gravity with negligible quantum corrections requires that the corresponding dual field theory has a large number of degrees of freedom. In the prototypical AdS/CFT duality [22] this is realized by taking the limit of a large number of colors $N_{C}$. In the light front, the bound-state dynamics corresponds to strongly correlated multiple-particle states in the Fock expansion, and the large $N_{C}$ limit is not a natural concept. The mapping of the AdS bound-state equations to the light-front Hamiltonian is carried out for $N_{C}=3$, with remarkable phenomenological success. Following the original holographic ideas $[23,24]$ it is thus tempting to conjecture that the required large number of degrees of freedom is provided in this case by the large number of Fock states in light-front dynamics. In fact, in the light-front approach, the effective potential is the result of integrating out all higher Fock states, corresponding to an infinite number of degrees of freedom, thus absorbing all the quantum effects. The reduction of higher-Fock states to an effective potential is not related to the value of $N_{C}$. It should also be noted that the main objective of this report, namely to find a bound state equation in QCD, is bound to incorporate an essential feature of $N_{C} \rightarrow \infty$ QCD: the feature that all bound states, also the excited ones, are stable.

There is another important relation which we have not fully exploited here: the relation between the generators of the one-dimensional conformal group and those of the isometries of Anti-de Sitter space in 2 dimensions, $\mathrm{AdS}_{2}$. The relations between the generators of the triple isomorphism of $\operatorname{Conf}\left(R^{1}\right), S O(2)$, and $\mathrm{AdS}_{2}$ are given in Secs. A.2.3 and 3.3. The connection between the isometries of $\mathrm{AdS}_{2}$ space and the $S O(2,1)$ group of conformal quantum mechanics is the basis of the $\mathrm{AdS}_{2} / \mathrm{CFT}_{1}$ correspondence [173]. In Chapter 3 we have shown that the introduction of the scale leading to a confining Hamiltonian corresponds to a "detuning" of the relation between the Hamiltonian and the $S O(2,1)$ transformations, see $(3.34)$. This detuning, which corresponds to the introduction of a scale and the appearance of a harmonic oscillator potential in the LF 
Hamiltonian, has a very simple geometrical interpretation [254] on the $\mathrm{AdS}_{2}$ hyperboloid embedded in a three-dimensional Euclidean space (Fig. A.1).

Recently, the relevance of $\mathrm{AdS}_{2}$ and the emergence of its IR one-dimensional dual quantum field theory [357] has become manifest through holographic renormalization in the bulk [129], the geometric version of the the Wilson renormalization group [128]. In this approach, the holographic flow in the bulk geometry from the boundary theory to the resulting low energy behavior is associated with the holographic coordinate which represents the energy scale [358]. Of particular interest is the formulation of holographic renormalization flows for strongly interacting theories with a cut-off in the bulk, where integration of high energy modes corresponds to integrate the bulk geometry up to some intermediate scale to extract the low-energy effective theory of the initial boundary theory [359, 360]. A particularly interesting example, and a line of research worth pursuing, is the holographic flow of boundary theories to $\mathrm{AdS}_{2}$ geometry in the infrared [360, 361, 362], since its dual one-dimensional conformal quantum field theory is realized as conformal quantum mechanics (Sect. 3.3) with remarkable features which, intertwined to light-front dynamics and holography as explained in this report, captures quite well important properties of the hadronic world. 


\section{Acknowledgements}

We would like to thank Fu-Guang Cao, Carl Carlson, Alexandre Deur, Stanislaw Glazek, Diego Hofman, Eberhard Klempt, Volkhard Müller, Joseph Polchinski, Craig Roberts, Robert Shrock, Matthew Strassler, Peter Tandy and Arkadiusz Trawiński for valuable discussions and communications. The work of SJB was supported by the Department of Energy contract DE-AC02-76SF00515. The work of JE was supported by the NSF under Grant PHY-1068008. 


\section{Addendum}

After completion of this work, it has become apparent that the striking phenomenological similarities between the baryon and meson spectra, described in this review, reflect underlying supersymmetric relations responsible for these important features. In fact, it has been shown recently that the extension of superconformal quantum mechanics to the light front has important applications to hadronic physics [363, 364].

In particular, it has been shown [363] that a comparison of the half-integer LF bound-state equations with the Hamiltonian equations of superconformal quantum mechanics $[172,365]$ fixes the form of the LF potential, in full agreement with the phenomenologically deduced form $V(\zeta)=\lambda_{B} \zeta$. This new development addresses one of the open problems of the approach to strongly-coupled QCD described in this report (See Sec. 8.1). In contrast to conformal quantum mechanics without supersymmetry, which is dual to the bosonic sector of AdS only up to a constant term, which in turn is fixed by embedding the LF wave equations for arbitrary integer spin into AdS. This procedure, originally developed by Fubini and Rabinovici [172], is the superconformal extension of the procedure applied by de Alfaro et al. [94]. Following this procedure a new evolution Hamiltonian is constructed from a generalized supercharge, which is a superposition of the original supercharge together with a spinor operator which occurs only in the superconformal algebra. The resulting one-dimensional effective theory applied to the fermionic LF bound-state equations is equivalent to the semiclassical approximation to strongly coupled dynamics which, as described in this report, follows from the lightfront clustering properties of the semiclassical approximation to strongly coupled-QCD dynamics and its holographic embedding in AdS space.

In Ref. [363] superconformal quantum mechanics was used to describe baryonic states. In this case, the supercharges relate the positive and negative chirality components of the baryon wave functions, consistent with parity conservation. Light-front superconformal quantum mechanics can also be used to relate hadronic states with different fermion number [364]. In this approach the nucleon trajectory is the super- 
partner of the pion trajectory, but the pion, which is massless in the chiral limit, has no supersymmetric partner. It is important to notice that the quantum-mechanical supersymmetric relations derived in $[363,364]$ are not a consequence of a supersymmetry of the underlying quark and gluon fields; they are instead a consequence of the superconformal-confining dynamics of the semiclassical theory described in this Review and the clustering inherent in light-front holographic QCD. 


\section{Appendix A}

\section{Riemannian Geometry and Anti-de Sitter Space}

We briefly review in this appendix relevant elements of Riemannian geometry useful in the discussion of Anti-de Sitter space and applications of the AdS/CFT correspondence.

\section{A.1 Basics of non-Euclidean geometry}

The geometric properties of a $D$-dimensional curved space with coordinates $x^{M}=$ $\left(x^{0}, x^{1} \cdots x^{D-1}\right)$ are described by the metric tensor $g_{M N}(x)$ which defines the space-time metric

$$
d s^{2}=g_{M N} d x^{M} d x^{N}
$$

in each reference frame. In D-dimensional Minkowski space in Cartesian coordinates, the metric tensor $\eta_{M N}$ has diagonal components $(1,-1, \cdots,-1)$. In non-Euclidean geometry the metric tensor varies from point to point and its form depends on the coordinate choice. Since (A.1) is invariant, the change of the metric induced by a coordinate transformation $x^{M} \rightarrow x^{M}(x)$

$$
d x^{M}=\frac{\partial x^{M}}{\partial x^{N}} d x^{N}=\partial_{N} x^{M} d x^{N}
$$

is

$$
g_{M N}^{\prime}\left(x^{\prime}\right)=\partial_{M}^{\prime} x^{R} \partial_{N}^{\prime} x^{S} g_{R S}(x),
$$


where we define $\partial_{M} \equiv \partial / \partial x^{M}, \partial_{M}^{\prime} \equiv \partial / \partial x^{M}$. A $D$-components object $V^{M}$ which transforms as the differential quantities (A.2) is said to be a contravariant vector

$$
V^{M}(x) \rightarrow V^{M}\left(x^{\prime}\right)=\partial_{N} x^{M} V^{N}
$$

The gradient $\partial_{M} \phi$ of a scalar function $\phi(x)=\phi^{\prime}\left(x^{\prime}\right)$ transforms according to

$$
\partial_{M}^{\prime} \phi^{\prime}\left(x^{\prime}\right)=\partial_{M}^{\prime} x^{N} \partial_{N} \phi(x)
$$

A $D$-components object $V_{M}$ which transforms as the gradient of a scalar function (A.5) is said to be a covariant vector

$$
V_{M}(x) \rightarrow V_{M}^{\prime}\left(x^{\prime}\right)=\partial_{M}^{\prime} x^{N} V_{N}
$$

Thus, contravariant vectors are denoted by upper components, and covariant ones by lower components. A covariant or contravariant or mixed tensor has several indices which transform according to (A.6) or (A.4). For example a covariant tensor of rank two transforms as

$$
V_{M N} \rightarrow V_{M N}^{\prime}=\partial_{M}^{\prime} x^{S} \partial_{N}^{\prime} x^{R} V_{R S}
$$

The inverse of $g_{M N}$ is the contravariant metric tensor $g^{M N} ; g_{M R} g^{R N}=\delta_{M}^{N}$, where $\delta_{M}^{N}$ is the mixed unit tensor: $\delta_{N}^{M}=1, N=M ; \delta_{N}^{M}=0, N \neq M$. In Minkowski space-time the inverse metric tensor in Cartesian coordinates is equal to the original one: $\eta_{M N}=\eta^{M N}$, but this is not the case for a general non-Euclidean metric. We can transform contravariant vectors in covariant ones and vice versa. Indices are lowered or raised by the metric tensor $V_{M}=g_{M N} V^{N}, V^{M}=g^{M N} V_{N}$. The scalar product of two vectors $A^{M}=\left(A^{0}, A^{1} \cdots A^{D-1}\right)$ and $B^{M}=\left(B^{0}, B^{1} \cdots B^{D-1}\right)$

$$
A \cdot B=A_{N} B^{M}=A^{M} B_{N}=g_{M N} A^{M} B^{N}=g^{M N} A_{M} B_{N},
$$

is invariant under coordinate transformations as one can easily verify from (A.4) and (A.6).

When integrating in a curved space time, the volume element $d V$ should behave as an invariant upon integration over a D-dimensional domain. In curved space

$$
d V=\sqrt{g} d x^{0} d x^{1} \cdots d x^{D-1}
$$


where $g$ is the absolute value of the metric determinant $g \equiv\left|\operatorname{det} g_{\mathrm{MN}}\right|$.

\section{A.1.1 Covariant derivative and parallel transport}

The next step is to define a covariant derivative $D_{M}$ which transforms covariantly. For example, when $D_{M}$ acts on a covariant vector $V_{N}$ the resulting rank-two covariant tensor $D_{M} V_{N}$ should transform as

$$
D_{M}^{\prime} V_{N}^{\prime}=\partial_{M}^{\prime} x^{R} \partial_{N}^{\prime} x^{S} D_{R} V_{S}
$$

This is not the case for the usual partial derivative

$$
\partial_{M}^{\prime} V_{N}^{\prime}=\partial_{M}^{\prime} x^{S} \partial_{N}^{\prime} x^{R} \partial_{S} V_{R}+V_{R} \partial_{M}^{\prime} \partial_{N}^{\prime} x^{R}
$$

since the second term spoils the general covariance unless the second derivatives vanish: $\partial^{\prime}{ }_{M} \partial^{\prime}{ }_{N} x^{\prime R}=0$. This happens only for linear transformations: $x^{M}=\Lambda_{N}^{M} x^{N}+a^{M}$, where $\Lambda_{N}^{M}$ and $a^{M}$ are constants. In non-Euclidean geometry a vector changes its components under parallel transport when comparing two vectors at the same point (for example when computing the change in the velocity of a particle). One has to make a parallel transport of a vector $V^{M}$ from $x^{M}$ to an infinitesimally close point $x^{M}+\epsilon^{M}$

$$
V^{M}(x) \rightarrow V^{M}(x+\epsilon)=V^{M}(x)+\Gamma_{K L}^{M} V^{K}(x) \epsilon^{L},
$$

where the $\Gamma_{K L}^{M}$, so-called Christoffel symbols for the connection, are functions of the coordinates. Taking into account parallel transport, the total change expressed by the covariant derivative is thus

$$
D_{M} V^{N}=\partial_{M} V^{N}+\Gamma_{K M}^{N} V^{K}
$$

The expression for the covariant derivative of a covariant vector follows from (A.14) and the fact that the scalar product of two vectors is invariant under parallel transport. The result is

$$
D_{M} V_{N}=\partial_{M} V_{N}-\Gamma_{M N}^{K} V_{K}
$$

It is possible to choose a local inertial coordinate system $x^{M}$ at a given point with metric $\eta_{M N}$. Under a coordinate transformation (A.3)

$$
g_{M N}^{\prime}=\partial_{M}^{\prime} x^{R} \partial_{N}^{\prime} x^{S} \eta_{R S}
$$


Differentiating (A.15) with respect to $x^{\prime K}$ and permuting indices

$$
\partial^{\prime}{ }_{M} \partial^{\prime}{ }_{N} x^{R} \partial^{\prime}{ }_{K} x^{S} \eta_{R S}=\frac{1}{2}\left(\partial^{\prime}{ }_{M} g_{N K}^{\prime}+\partial_{N}^{\prime} g_{M K}^{\prime}-\partial_{K}^{\prime} g_{M N}^{\prime}\right)
$$

From the inverse transformation of $g_{M N}$ to $\eta_{M N}$ and the relation $\delta_{M}^{N}=\partial^{\prime}{ }_{M} x^{R} \partial_{R} x^{\prime N}$, we find the useful relation

$$
\partial^{\prime}{ }_{M} \partial^{\prime}{ }_{N} x^{R}=\frac{1}{2} g^{\prime K L}\left(\partial_{M}^{\prime} g_{N L}^{\prime}+\partial_{N}^{\prime} g_{M L}^{\prime}-\partial_{L}^{\prime} g_{M N}^{\prime}\right) \partial^{\prime}{ }_{K} x^{R}
$$

Using (A.17) it is straightforward to prove that the covariant derivative has indeed the right transformation properties under a general coordinate transformation (A.10). Furthermore, we find from (A.17) and (A.14) an expression for the the Christoffel symbols $\Gamma_{M N}^{K}$ in terms of the metric tensor

$$
\Gamma_{M N}^{K}=\frac{1}{2} g^{K L}\left(\partial_{M} g_{N L}+\partial_{N} g_{M L}-\partial_{L} g_{M N}\right)
$$

where $\Gamma_{M N}^{K}=\Gamma_{N M}^{K}$. Eq. (A.18) can also be obtained from the condition that the covariant derivative of the metric tensor $g_{M N}$ is zero

$$
D_{K} g_{M N}=0
$$

It follows from (A.18) that $\Gamma_{M R}^{R}=\frac{1}{2} g^{R S} \partial_{M} g_{R S}=\partial_{M} \ln \sqrt{g}$, where $g$ is the absolute value of the metric determinant $g \equiv\left|\operatorname{det} g_{\mathrm{MN}}\right|$. We thus find the expression for the divergence of a vector in curved space-time

$$
D_{M} V^{M}=\frac{1}{\sqrt{g}} \partial_{M}\left(\sqrt{g} V^{M}\right)
$$

It also follows from the symmetry of the $\Gamma_{M N}^{K}$ that the rank-two antisymmetric tensor $D_{M} V_{N}-D_{N} V_{M}=\partial_{M} V_{N}-\partial_{N} V_{M}$.

\section{A.1.2 Space-time curvature}

Unlike flat space, the second covariant derivative in curved space depends on the order of derivation:

$$
\left[D_{N}, D_{K}\right] V_{M}=-R_{M N K}^{L} V_{L},
$$


where the fourth-order Riemann tensor or curvature tensor $R_{M N K}^{L}$,

$$
R_{M N K}^{L}=\partial_{N} \Gamma_{M K}^{L}-\partial_{K} \Gamma_{M N}^{L}+\Gamma_{N R}^{L} \Gamma_{M K}^{R}-\Gamma_{K R}^{L} \Gamma_{M N}^{R},
$$

depends on the coordinate system chosen. Likewise for a contravariant vector one obtains

$$
\left[D_{N}, D_{K}\right] V^{M}=R_{L N K}^{M} V^{L}
$$

The curvature tensor in antisymmetric in the indices $N$ and $K ; R_{M N K}^{L}=-R_{M K N}^{L}$. The tensor is null in Euclidean space $R_{M N K}^{L}=0$, and conversely, if $R_{M N K}^{L}=0$ the space is Euclidean. Is is useful to express the Riemann tensor in covariant form

$$
R_{K M L N}=g_{K R} R_{M L N}^{R}
$$

with the symmetry properties

$$
\begin{aligned}
& R_{K M L N}=-R_{M K L N}, \\
& R_{K M L N}=-R_{K M N L}, \\
& R_{K M L N}=R_{L N K M} .
\end{aligned}
$$

The rank two tensor

$$
R_{M N}=g^{K L} R_{K M L N}=R_{M L N}^{L}
$$

is the Ricci tensor $R_{M N}=R_{N M}$. Contracting $R_{M N}$ we obtain the scalar curvature $\mathcal{R}$

$$
\mathcal{R}=g^{M N} R_{M N}=g^{K L} g^{M N} R_{K M L N}
$$

which encodes intrinsic properties of space-time. The Einstein tensor given by

$$
G_{M N}=R_{M N}-\frac{1}{2} \mathcal{R} g_{M N}
$$

has the property that $D_{N} G_{M}^{N}=0$, known as the Bianchi identity.

To have a better intuition of parallel transport and the Ricci tensor consider the parallel transport of a vector between two points along different paths in a non Euclidean 
space. In particular the change $\Delta V_{M}$ of a vector along a closed infinitesimal loop is

$$
\Delta V_{M}=\oint \Gamma_{M N}^{L} V_{L} d x^{N}
$$

Using Stokes theorem one obtains

$$
\Delta V_{M}=\frac{1}{2} R_{M N K}^{L} V_{L} \Delta S^{N K}
$$

where $\Delta S^{N K}$ is the infinitesimal area enclosed by the integration contour. The result of parallel transporting a vector is path dependent.

\section{A.1.3 Spinors in non-Euclidean geometry}

To describe spin in curved space-time we attach an orthonormal frame, the vielbein, at each point in space-time [366]. The vielbein at a point $P$ is a local inertial system. The spin connection gives us information on how the vielbein is rotated when it moves along a curve. It turns out that the vielbein is essential for treating spinors in non-Euclidean geometry. ${ }^{1}$

Let $\xi^{A}(x)$ be the coordinates of the point $P$ in the local inertial frame. The vielbein is a set of $D$ orthonormal tangent vectors $e_{M}^{A}(x)=\partial_{M} \xi^{A}(x)$, where $x$ are the coordinates of the same point $P$ in a general frame with metric tensor $g_{M N}(x)$, and the index $A$ is a Lorentz index in tangent space. Lorentz indices are denoted by $A, B \cdots$ and curved space indices by $M, N \cdots$. Since the metric tensor in the inertial frame is the Minkowski tensor $\eta_{M N}$, the metric tensor in the general frame is according to (A.3) $g_{M N}(x)=\partial_{M} \xi^{A} \partial_{N} \xi^{B} \eta_{A B}$. Thus

$$
g_{M N}(x)=\eta_{A B} e_{M}^{A}(x) e_{N}^{B}(x)=e_{M}^{A}(x) e_{A N}(x)
$$

where $e_{A M}=\eta_{A B} e_{M}^{B}$, since the tangent indices are raised or lowered by the Minkowski metric $\eta_{A B}$. The inverse vielbein is denoted $e_{A}^{M}$, thus $e_{M}^{A}(x) e_{B}^{M}(x)=\delta_{B}^{A}$. The vectors $e_{M}^{A}$ form a complete basis at each point $P$. The vielbein is not uniquely determined since

\footnotetext{
${ }^{1}$ Technically the quantities $\partial x^{M} / \partial x^{N}$ in (A.4) for transforming a vector field $V^{M}$ are the elements of the general linear group $G L(D, R)$, the group of invertible real $D \times D$ matrices. Thus a vector $V^{M}$ transforms in the fundamental representation of $G L(D, R)$. Spinors transform under the special orthogonal group $S O(D)$, a subgroup of $G L(D, R)$, but the spinor representations are not comprised in the representations of $G L(D, R)$. The vielbein formalism allows us to replace the $G L(D, R)$ matrices by a $S O(D)$ matrix representation.
} 
in each point there is an infinity of equivalent inertial coordinate systems, all related through Lorentz transformations; $e_{M}^{\prime A}(x)=\Lambda_{B}^{A}(x) e_{M}^{B}(x)$, where $\Lambda_{B}^{A}(x)$ is a local Lorentz transformation at the point $P$.

For spinors, the analogue of the Christoffel symbols is the spin connection denoted $\omega_{M}^{A}$. Thus the covariant derivative of a vector with tangent indices $V^{A}=e_{M}^{A} V^{M}$

$$
D_{M} V^{A}=\partial_{M} V^{A}+\omega_{M}^{A}{ }_{B} V^{B}
$$

Consistency with (A.19) and (A.33) implies that the covariant derivative of the vielbein is zero $^{2}$

$$
D_{M} e_{N}^{A}=0,
$$

and thus $D_{M} e_{N}^{A}=\partial_{M} e_{N}^{A}-\Gamma_{M N}^{L} e_{L}^{A}+\omega_{M}^{A}{ }_{B} e_{N}^{B}=0$. The spin connection then follows from (A.35)

$$
\omega_{M}^{A B}=e_{N}^{A} \partial_{M} e^{N B}+e_{L}^{A} e^{N B} \Gamma_{N M}^{L}
$$

Equations (A.14) and (A.34) for the covariant derivative are equivalent. To see this we contract (A.34) with $e_{A}^{N}$ and use (A.36):

$$
\begin{aligned}
D_{M} V^{N}=e_{A}^{N} D_{M} V^{A} & =e_{A}^{N} \partial_{M} V^{A}+e_{A}^{N} e_{K}^{A}\left(\partial_{M} e_{B}^{K}\right) V^{B}+e_{A}^{N} e_{L}^{A} e_{B}^{K} \Gamma_{K M}^{L} V^{B} \\
& =\partial_{M}\left(e_{A}^{N} V^{A}\right)-\left(\partial_{M} e_{A}^{N}\right) V^{A}+\left(\partial_{M} e_{B}^{N}\right) V^{B}+\Gamma_{K M}^{N}\left(e_{B}^{K} V^{B}\right) \\
& =\partial_{M} V^{N}+\Gamma_{K M}^{N} V^{K} .
\end{aligned}
$$

We now come to spinors. We consider a spinor field $\psi(x)$ in the spinor representation of the Lorentz group. The generators of the Lorentz group $\Sigma_{A B}$ in the spinor representation are

$$
\Sigma_{A B}=\frac{i}{4}\left[\Gamma_{A}, \Gamma_{B}\right]
$$

where the flat space gamma matrices $\Gamma_{A}$ are constant matrices obeying the usual anticommutation relations

$$
\left\{\Gamma^{A}, \Gamma^{B}\right\}=2 \eta^{A B} .
$$

The covariant derivative of $\psi$ is defined by

$$
D_{M} \psi=\left(\partial_{M}-\frac{i}{2} \omega_{M}^{A B} \Sigma_{A B}\right) \psi .
$$

\footnotetext{
${ }^{2}$ The condition (A.35) corresponds to minimal coupling of the spinors. Deviations from minimal coupling are measured by the torsion $T_{M N}^{A}$ defined by $T_{M N}^{A}=D_{M} e_{N}^{A}-D_{N} e_{M}^{A}$.
} 
Under a local Lorentz transformation $\psi(x) \rightarrow \Lambda(x) \psi(x)$, with $\Lambda=\exp \left(-\frac{i}{2} \omega_{A B} \Sigma^{A B}\right)$, the covariant derivative $D_{M} \psi(x)$ also transforms as a spinor, $D_{M} \psi(x) \rightarrow \Lambda(x) D_{M} \psi(x)$, thus general covariance is maintained.

The vielbein allows us also to construct space-dependent $\Gamma$-matrices in non-Euclidean space

$$
\Gamma^{M}(x)=e_{A}^{M}(x) \Gamma^{A} .
$$

From (A.33) and (A.38) it follows that the matrices $\Gamma^{M}$ obey $\left\{\Gamma^{M}(x), \Gamma^{N}(x)\right\}=$ $2 g^{M N}(x)$. It can also be proven from (A.35) that the matrices $\Gamma^{M}(x)$ are covariantly constant, $\left[D_{M}, \Gamma^{N}(x)\right]=0$. Thus the Dirac operator $\not D$ can be defined as

$$
\not D=\Gamma^{M}(x) D_{M}=e_{A}^{M}(x) \Gamma^{A} D_{M} .
$$

\section{A.2 Maximally symmetric spaces}

\section{A.2.1 Definition}

A space-time manifold is said to be maximally symmetric if it has the same number of isometries as Euclidean space. A $D$-dimensional Euclidean space is isotropic and homogeneous. Its metric tensor is invariant under $D(D-1) / 2$ rotations (isotropy) and $D$ translations (homogeneity). We have thus altogether $D(D+1) / 2$ isometries i.e., transformations which leave the metric invariant. This is also the maximal number of isometries of a $D$-dimensional Minkowski space, therefore flat space-time is a maximally symmetric space. This concept of isometry and maximal symmetry can be translated into non-Euclidean geometry.

If the metric does not change its form under a coordinate transformation $x \rightarrow x^{\prime}$, that is $g_{M N}(x) \rightarrow g_{M N}^{\prime}\left(x^{\prime}\right)=g_{M N}\left(x^{\prime}\right)$, then it follows from (A.3) that

$$
g_{M N}\left(x^{\prime}\right)=\partial_{M}^{\prime} x^{R} \partial_{N}^{\prime} x^{S} g_{R S}(x) .
$$

Any change of coordinates that satisfies A.42 is an isometry. In particular, if one looks for local isometries under infinitesimal transformations: $x^{\prime M}=x^{M}+\epsilon \xi^{M}(x)$, one can show easily that the requirement to be an isometry is given by the covariant structure

$$
D_{M} \xi_{N}+D_{N} \xi_{M}=0,
$$

the Killing equation. Any vector field $\xi_{M}(x)$ satisfying (A.43) is said to form a Killing 
vector of the metric $g_{M N}(x)$. The maximum number of independent Killing vectors in a $D$-dimensional space is $D(D+1) / 2$, thus the Killing vectors are the infinitesimal generators of isometries. A space with a metric that admits this maximal number of Killing vectors is maximally symmetric: it is homogeneous and isotropic for every point.

\section{A.2.2 Anti-de Sitter space-time $\operatorname{AdS}_{d+1}$}

Anti-de Sitter space is the maximally symmetric space-time with negative scalar curvature. We can construct the metric of a maximally symmetric space by embedding it in a flat space of one dimension higher. Anti-de Sitter space-time in $d+1$ dimensions, $\mathrm{AdS}_{d+1}$, can be described as a hyperboloid embedded in a flat $d+2$ dimensional spacetime with an additional time-like direction, i.e. the surface

$$
X_{-1}^{2}+X_{0}^{2}-X_{1}^{2}-\cdots-X_{d}^{2} \equiv \sum_{M=-1, d} X^{M} X_{M}=R^{2}
$$

with metric induced by the $(d+2)$-dimensional flat metric with signature $(2, d)$

$$
d s^{2}=d X_{-1}^{2}+d X_{0}^{2}-d X_{1}^{2}-\cdots-d X_{d}^{2}
$$

The isometries of the embedded space are those which leave the hyperboloid invariant; that is, they are isomorphic to the group $S O(2, d)$. This group in turn is isomorphic to the conformal group $\operatorname{Conf}\left(R^{1, d-1}\right)$. These groups have have $d(d+1) / 2$ generators. The isometries of $\mathrm{AdS}_{d+1}$ can be either obtained via the Killing vectors (A.43), or, expressed in the embedding coordinates $X$ through the transformations of $S O(2, d)$.

The AdS space time contains closed time-like curves. For example, for fixed coordinates $\left\{X_{1}, \ldots, X_{d}\right\}$, any closed path along the circle in the $\left(X_{0}, X_{1}\right)$ plane is a closed time-like curve. Global coordinates (i.e. coordinates which cover the entire space time) are $\rho, \tau$, and $\Omega_{i}$ with $0 \leq \rho<\pi / 2,-\pi<\tau \leq \pi$, and $-1 \leq \Omega_{i} \leq 1$ with $\sum_{i=1}^{d} \Omega_{i}^{2}=1$. Global coordinates are related to the embedding coordinates $X$ by:

$$
\begin{aligned}
X_{-1} & =R \frac{\sin \tau}{\cos \rho}, \\
X_{0} & =R \frac{\cos \tau}{\cos \rho} \\
X_{i} & =R \Omega_{i} \tan \rho .
\end{aligned}
$$


In global coordinates the metric is

$$
d s^{2}=\frac{R^{2}}{\cos ^{2} \rho}\left(d \tau^{2}-d \rho^{2}-\sin ^{2} \rho d \Omega^{2}\right) .
$$

The universal cover of Anti-de Sitter space is obtained by unwrapping the hyperboloid along the time-like circle and repeatedly gluing the resulting space-time to itself along the seam ad infinitum in order to eliminate the periodicity, which also eliminates the closed time like curves.

Poincaré coordinates naturally split the AdS space time into smaller patches. The plane $X_{-1}=X_{d}$ splits the full $\mathrm{AdS}_{d+1}$ space in two patches. On these patches we can introduce coordinates by first defining the light-cone coordinates

$$
u \equiv \frac{1}{R^{2}}\left(X_{-1}-X_{d}\right), \quad v \equiv \frac{1}{R^{2}}\left(X_{-1}+X_{d}\right)
$$

and by introducing the Poincaré coordinates $x^{0}=t, x^{i}, i=1 \ldots d-1$ and $z$, which are related to the embedding coordinates by

$$
x^{i}=\frac{X_{i}}{R u}, \quad x^{0}=\frac{X_{0}}{R u}, \quad z=\frac{1}{u} .
$$

In order to obtain the metric tensor in the Poincare coordinates we eliminate $v$ by the embedding condition (A.44) and obtain

$$
\begin{aligned}
X_{-1} & =\frac{1}{2 z}\left(z^{2}+R^{2}+\mathbf{x}^{2}-t^{2}\right), \\
X_{0} & =\frac{R t}{z} \\
X_{i} & =\frac{R x^{i}}{z} \\
X_{d} & =\frac{1}{2 z}\left(z^{2}-R^{2}+\mathbf{x}^{2}-t^{2}\right) .
\end{aligned}
$$

The coordinate $z=R^{2} /\left(X_{-1}-X_{d}\right)$ is referred to as the holographic coordinate, and separates the AdS space-time into two distinct regions: $z>0$ and $z<0$. Each region is absent of closed time-like curves. The region $z=0$ belongs to the boundary of the AdS space time, and we will be interested in the Poincaré patch $z>0$. In Poincaré coordinates the AdS metric with coordinates $x^{M}=\left(x^{\mu}, z\right), \mu, \nu=0,1,2, \cdots, d-1$, takes 
the form

$$
d s^{2}=g_{M N} d x^{M} d x^{N}=\frac{R^{2}}{z^{2}}\left(\eta_{\mu \nu} d x^{\mu} d x^{\nu}-d z^{2}\right)
$$

and thus

$$
g_{M N}=\frac{R^{2}}{z^{2}} \eta_{N M}, \quad g^{M N}=\frac{z^{2}}{R^{2}} \eta^{N M}, \quad e_{M}^{A}=\frac{R}{z} \delta_{M}^{A}, \quad e_{A}^{M}=\frac{z}{R} \delta_{A}^{M},
$$

where $\eta_{N M}$ has diagonal components $(1,-1, \cdots,-1)$. The metric determinant $g=$ $\left|\operatorname{det} g_{M N}\right|$ is $g=(R / z)^{2 d+2}$. Additional details regarding various coordinates on anti-de Sitter space time and its Poincaré patches can be found in Ref. [367].

The Christoffel symbols (A.18) for the metric (A.57) are:

$$
\begin{aligned}
\Gamma_{M N}^{L} & =\frac{1}{2} g^{L K}\left(\partial_{M} g_{K N}+\partial_{N} g_{K M}-\partial_{K} g_{M N}\right) \\
& =\frac{-1}{z}\left(\delta_{M}^{z} \delta_{N}^{L}+\delta_{N}^{z} \delta_{M}^{L}-\eta^{L z} \eta_{M N}\right),
\end{aligned}
$$

and the covariant derivative (A.14) for a vector is

$$
\begin{aligned}
D_{z} V_{M} & =\left(\partial_{z}+\frac{1}{z}\right) V_{M}, \\
D_{\mu} V_{z} & =\partial_{\mu} V_{z}+\frac{1}{z} V_{\mu}, \\
D_{\mu} V_{\nu} & =\partial_{\mu} V_{\nu}+\frac{1}{z} \eta_{\mu \nu} V_{z} .
\end{aligned}
$$

Every maximally symmetric space has constant curvature $\mathcal{R}$. In fact, for an isotropic and homogeneous space the Ricci tensor $R_{M N K}^{L}$ can only depend on the metric tensor, and thus by virtue of its symmetry properties (A.25) - (A.27) it should have the form $R_{K M L N}=\lambda\left(g_{K L} g_{M N}-g_{K N} g_{M L}\right)$, where $\lambda$ is a constant. For $\mathrm{AdS}_{d+1}$ the Ricci tensor (A.28) is $R_{M N}=\lambda d g_{M N}$ and the scalar curvature (A.29) $\mathcal{R}=d(d+1) \lambda$. A simple but tedious computation using the expression (A.22) for the Riemann tensor and (A.58) for the Christoffel symbols gives $\mathcal{R}=\eta^{z z} d(d+1) / R^{2}$ and thus ${ }^{3}$

$$
R_{K M L N}=-\frac{1}{R^{2}}\left(g_{K L} g_{M N}-g_{K N} g_{M L}\right)
$$

From the realization of $\mathrm{AdS}_{d+1}$ as an hyperboloid in a $d+2$ dimensional space, (See Eq. (A.44)), it follows that the isometries of $\mathrm{AdS}_{d+1}$ are isomorphic to the trans-

\footnotetext{
${ }^{3}$ This relation shows that the signum of the holographic variable in the metric determines the sign of the scalar curvature and hence if the space is a de Sitter or an anti-de Sitter space.
} 
formations which leave this hyperboloid invariant, i.e. the elements of $S O(2, d)$. The Killing vectors of $A d S_{d+1}$ in Poincaré coordinates can be constructed from the $S O(2, d)$ generators

$$
L^{M, N}=i\left(X^{N} \frac{\partial}{\partial X_{M}}-X^{M} \frac{\partial}{\partial X_{N}}\right),
$$

where $M, N=-1,0, \ldots d$, with the relations (A.52) - (A.55) between the different coordinates. In the next subsection this is done for the case $d=1$.

\section{A.2.3 Relation between $\operatorname{Conf}\left(R^{1}\right), S O(2,1)$ and the isometries of $\mathrm{AdS}_{2}$}

Since the conformal group plays an eminent role in our treatment, we give here a more extensive discussion of the relation between the generators of the conformal group and those of the isometries of Anti-de Sitter space in two dimensions, $\mathrm{AdS}_{2}{ }^{4}$. In Figure A.1 we show the embedding of the $\mathrm{AdS}_{2}$, i.e. $d=1$, into a three dimensional space. The surface of the hyperboloid is the space $\mathrm{AdS}_{2}$. The intersection with the plane $X_{1}-X_{-1}=0$ corresponds to the value $z= \pm \infty$ which separates the $\mathrm{AdS}_{2}$ space into two patches, none of them containing time-like closed curves. The value $z=0$ correspond to the limit $X_{1} \rightarrow-\infty$.

The conformal group in one dimension $\operatorname{Conf}\left(R^{1}\right)$ is locally isomorphic not only to the group $S O(2,1)$, but also to the isometries of $\mathrm{AdS}_{2}$. The three generators of the latter, $A^{(i)}$, can be constructed with the Killing vectors $\xi^{(i)}$ as (See Sec. A.2.1)

$$
A^{(i)}=\xi^{(i) M} \frac{\partial}{\partial x^{M}}, \quad x^{1}=t, x^{2}=z
$$

The conditions for the Killing vectors of $\mathrm{AdS}_{2}$ follow from the Killing equation (A.43)

$$
\partial_{t} \xi^{(i) 1}-\frac{1}{z} \xi^{(i) 2}=0, \quad \partial_{z} \xi^{(i) 2}-\frac{1}{z} \xi^{(i) 1}=0, \quad \partial_{t} \xi^{(i) 2}-\partial_{z} \xi^{(i) 1}=0
$$

with the solutions

$$
\xi^{(1)}=R(1,0), \quad \xi^{(2)}=(t, z), \quad \xi^{(3)}=\frac{1}{R}\left(\left(t^{2}+z^{2}\right), 2 t z\right) .
$$

The relation between the Killing vectors and the generators of $S O(2,1)$ is not

\footnotetext{
${ }^{4}$ The relation between the conformal group, $\mathrm{AdS}_{2}$ and the generators of $S O(2,1)$ is described with great detail in the Senior Thesis of T. Levine at Brown University [368]. We thank Antal Jevicki for pointing us this work.
} 


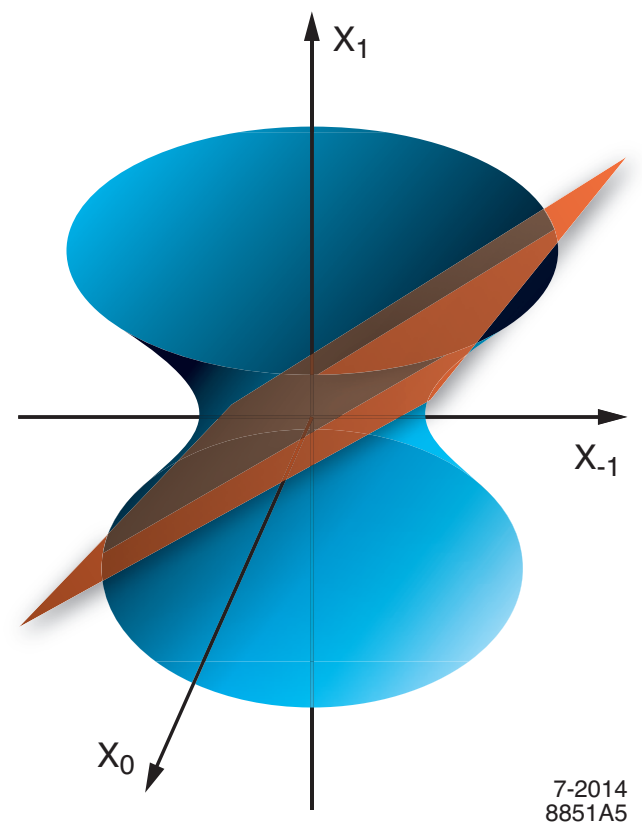

Figure A.1: The space $\mathrm{AdS}_{2}$ embedded as the hyperboloid $X_{-1}^{2}+X_{0}^{2}-X_{1}^{2}=R^{2}$ into a three dimensional space and the plane $X_{1}-X_{-1}=0$.

uniquely fixed by the commutation relations (A.43), therefore it is advantageous to construct them explicitly by going back to $\mathrm{AdS}_{2}$ as a 2-dimensional hyperboloid embedded in a three dimensional space (Fig. A.1). The embedding space has two time coordinates $\left\{X_{-1}, X_{0}\right\}$ and one space coordinate $X_{1}$. The space $\mathrm{AdS}_{2}$ is given by the surface $X_{-1}^{2}+X_{0}^{2}-X_{1}^{2}=R^{2}$ and its isometries are the transformations which leave the hyperboloid invariant, that is the rotation $L^{0,-1}$ in the $\left(X_{0}, X_{-1}\right)$ plane and the two boosts $L^{1,0}$ and $L^{1,-1}$. They obey the commutation relations of the $\operatorname{SO}(2,1)$ algebra (Sect. 3.1)

$$
\begin{aligned}
{\left[L^{0,-1}, L^{1,0}\right] } & =i L^{1,-1} \\
{\left[L^{0,-1}, L^{1,-1}\right] } & =-i L^{1,0} \\
{\left[L^{1,0}, L^{1,-1}\right] } & =-i L^{0,-1} .
\end{aligned}
$$

The Poincaré coordinates are given by (See (A.50)-(A.55))

$$
t=\frac{X_{0} R}{X_{-1}-X_{1}}, \quad z=\frac{R^{2}}{X_{-1}-X_{1}} .
$$

The relation between the generators of $S O(2,1)$ and the isometries of $\mathrm{AdS}_{2}$ given by 
(A.62), can now be obtained directly by expressing the rotation and the boost generators in Poincaré coordinates

$$
\begin{aligned}
& L^{0,-1}=i\left(X_{-1} \frac{\partial}{\partial X_{0}}-X_{0} \frac{\partial}{\partial X_{-1}}\right)=\frac{i}{2}\left\{\left(\frac{t^{2}+z^{2}}{R}+R\right) \partial_{t}+2 \frac{t z}{R} \partial_{z}\right\}, \\
& L^{1,0}=i\left(X_{0} \frac{\partial}{\partial X_{1}}+X_{1} \frac{\partial}{\partial X_{0}}\right)=\frac{i}{2}\left\{\left(\frac{t^{2}+z^{2}}{R}-R\right) \partial_{t}+2 \frac{t z}{R} \partial_{z}\right\}, \\
& L^{1,-1}=i\left(X_{-1} \frac{\partial}{\partial X_{1}}+X_{1} \frac{\partial}{\partial X_{-1}}\right)=i\left(t \partial_{t}+z \partial_{z}\right),
\end{aligned}
$$

where $X_{0}=X^{0}, X_{-1}=X^{-1}$ and $X_{1}=-X^{1}$. In terms of the generators of the isometries of $\mathrm{AdS}_{2}$ (A.62),

$$
L^{0,-1}=\frac{i}{2}\left(A^{(3)}+A^{(1)}\right), \quad L^{1,0}=\frac{i}{2}\left(A^{(3)}-A^{(1)}\right), \quad L^{1,-1}=i A^{(2)} .
$$

Notice that the asymmetry between the representation of the two boosts is due to the choice of the Poincaré coordinates in (A.66). In Table A.1 the relation between the generators of the isometries of $\mathrm{AdS}_{2}$, the generators of $S O(2,1)$ and the generators of the conformal group $H, D$, and $K$ (See Chapter 3) are displayed.

\begin{tabular}{lll}
\hline \hline $\operatorname{Conf}\left(R^{1}\right)$ & \multicolumn{1}{c}{$S O(2,1)$} & \multicolumn{1}{c}{$\operatorname{AdS}_{2}$} \\
\hline$H$ & $\frac{1}{a}\left(L^{0,-1}-L^{1,0}\right)$ & $\frac{i}{a} A^{(1)}=\frac{i R}{a} \partial_{t}$ \\
$D$ & $L^{1,-1}$ & $i A^{(2)}=i\left(t \partial_{t}+z \partial_{z}\right)$ \\
$K$ & $a\left(L^{0,-1}+L^{1,0}\right)$ & $i a A^{(3)}=\frac{i a}{R}\left(\left(t^{2}+z^{2}\right) \partial_{t}+2 z t \partial_{z}\right)$ \\
\hline \hline
\end{tabular}

Table A.1: Relation between the generators of the conformal group in one dimension Conf $\left(R^{1}\right)$, the generators of $S O(2,1)$ and the Killing vectors of $\mathrm{AdS}_{2}$ expressed in Poincaré coordinates.

The generators of the $\mathrm{AdS}_{2}$ isometries depend on the $\mathrm{AdS}_{2}$ Poincaré coordinates $t$ and $z$. Using Table A.1, one can see explicitly the equivalence of the generators of $\mathrm{AdS}_{2}$ isometries at the $\mathrm{AdS}_{2}$ boundary, $z=0$, with the representation of the conformal generators $H, D$ and $K$ in conformal quantum mechanics given by (C.14) in Appendix $\mathrm{C}: H=i \partial_{t}, D=i t \partial_{t}$ and $H=i t^{2} \partial_{t}$, provided that $a=R$. 


\section{Appendix B}

\section{Light-Front Metric Conventions and Spinors}

The Minkowski metric is written in terms of light-front coordinates

$$
d \sigma^{2}=d x^{+} d x^{-}-d \mathbf{x}_{\perp}^{2},
$$

with time-like and space-like components $x^{+}=x^{0}+x^{3}$ and $x^{-}=x^{0}-x^{3}$ respectively. We write contravariant four-vectors such as $x^{\mu}$ as

$$
x^{\mu}=\left(x^{+}, x^{-}, x^{1}, x^{2}\right)=\left(x^{+}, x^{-}, \mathbf{x}_{\perp}\right) .
$$

Scalar products are

$$
\begin{aligned}
x \cdot p & =x_{\mu} p^{\nu}=\mathrm{g}_{\mu \nu} x^{\mu} p^{\nu} \\
& =x_{+} p^{+}+x_{-} p^{-}+x_{1} p^{1}+x_{2} p^{2} \\
& =\frac{1}{2}\left(x^{+} p^{-}+x^{-} p^{+}\right)-\mathbf{x}_{\perp} \cdot \mathbf{p}_{\perp},
\end{aligned}
$$

with front-form metrics

$$
\mathrm{g}_{\mu \nu}=\left(\begin{array}{cccc}
0 & \frac{1}{2} & 0 & 0 \\
\frac{1}{2} & 0 & 0 & 0 \\
0 & 0 & -1 & 0 \\
0 & 0 & 0 & -1
\end{array}\right), \quad \mathrm{g}^{\mu \nu}=\left(\begin{array}{cccc}
0 & 2 & 0 & 0 \\
2 & 0 & 0 & 0 \\
0 & 0 & -1 & 0 \\
0 & 0 & 0 & -1
\end{array}\right) .
$$


A covariant vector such as $\partial_{\mu}$ is

$$
\partial_{\mu}=\left(\partial_{+}, \partial_{-}, \partial_{1}, \partial_{2}\right)=\left(\partial_{+}, \partial_{-}, \vec{\partial}_{\perp}\right)
$$

Thus $\partial^{+}=2 \partial_{-}$and $\partial^{-}=2 \partial_{+}$.

Useful Dirac matrix elements for light-front helicity spinors with spin component along the $z$-axis $(\lambda= \pm 1)$ are $[159]$

$$
\begin{aligned}
\bar{u}(p) \gamma^{+} u(q) \delta_{\lambda_{p}, \lambda_{q}} & =2 \sqrt{p^{+} q^{+}} \\
\bar{u}(p) \gamma^{+} u(q) \delta_{\lambda_{p},-\lambda_{q}} & =0
\end{aligned}
$$

with $\bar{v}_{\alpha}(\ell) \gamma^{\mu} v_{\beta}(k)=\bar{u}_{\beta}(k) \gamma^{\mu} u_{\alpha}(\ell)$. Other useful matrix elements for LF spinors are given in [159]. 


\section{Appendix C}

\section{Notes on Conformal Quantum Mechanics}

In this appendix we examine specific features of the framework introduced by de Alfaro et al. [94] which are useful for the discussions in Chapter 3. For the relation between the conformal group and the isometries of $\mathrm{AdS}_{2}$ see Sec. (A.2.3). We start with the dAFF action

$$
A=\frac{1}{2} \int d t\left(\dot{Q}^{2}-\frac{g}{Q^{2}}\right),
$$

and the corresponding Lagrangian

$$
L[Q]=\frac{1}{2}\left(\dot{Q}^{2}-\frac{g}{Q^{2}}\right),
$$

which is, up to a total derivative, conformally invariant i.e.,

$$
L\left[Q^{\prime}\right] d t^{\prime}=\left(L[Q]-\frac{d \Omega[Q]}{d t}\right) d t
$$

We use the notation $\dot{Q}=d Q(t) / d t, \quad \dot{Q}^{\prime}=d Q^{\prime}\left(t^{\prime}\right) / d t^{\prime}$. To proof (C.3) we perform a general conformal transformation

$$
t^{\prime}=\frac{\alpha t+\beta}{\gamma t+\delta}, \quad Q^{\prime}\left(t^{\prime}\right)=\frac{Q(t)}{\gamma t+\delta}, \quad \alpha \delta-\beta \gamma=1,
$$

with

$$
d t^{\prime}=\frac{d t}{(\gamma t+\delta)^{2}}, \quad \dot{Q}^{\prime}\left(t^{\prime}\right)=(\delta+\gamma t) \dot{Q}-\gamma Q .
$$


One obtains immediately

$$
A=\frac{1}{2} \int d t^{\prime}\left(\left(\dot{Q}^{\prime}\right)^{2}-\frac{g}{{Q^{\prime}}^{2}}\right)=\frac{1}{2} \int d t\left(\dot{Q}^{2}-\frac{g}{Q^{2}}-\frac{d \Omega}{d t}\right)
$$

with

$$
\Omega=\frac{1}{2} \gamma \frac{Q^{2}}{\delta+\gamma t}
$$

Therefore the action $A$ (C.1) is invariant, up to a surface term, under the group of conformal transformations in one dimension.

The constants of motion, which follow from the invariance of the action (C.1) under conformal transformations, are also the generators of the conformal group expressed in terms of the field operators $Q$. The conserved generators can be obtained from Noether's theorem. If the Lagrangian is form invariant under infinitesimal transformations i.e.,

$$
L^{\prime}\left[Q^{\prime}\right]=L\left[Q^{\prime}\right]+\frac{d \Omega}{d t}
$$

then $J$ obtained from

$$
\delta J=\left(L-\frac{\partial L}{\partial \dot{Q}} \dot{Q}\right) \delta t+\frac{\partial L}{\partial \dot{Q}} \delta Q+\Omega
$$

is a constant of motion.

The Noether currents of the three independent transformations of the conformal group in one dimension are:

1. For translations $t^{\prime}-t=\delta t=\epsilon$ and $\delta Q=0$ in (C.4). The group parameters are $\alpha=\delta=1, \gamma=0, \beta=\epsilon$. The Noether current is $J \epsilon=\left(L-\frac{\partial L}{\partial \dot{Q}} \dot{Q}\right) \epsilon$ and the translation operator in the variable $t$

$$
H=-J=\frac{1}{2}\left(\dot{Q}^{2}+\frac{g}{Q^{2}}\right)
$$

2. For dilatations $t^{\prime}-t=\delta t=\epsilon t$ and $\delta Q=Q^{\prime}-Q=\frac{1}{2} \epsilon Q+O\left(\epsilon^{2}\right)$ in (C.4). The group parameters are $\alpha=\sqrt{1+\epsilon}, \delta=1 / \sqrt{1+\epsilon}, \gamma=\beta=0$. The Noether current is $J \epsilon=\left(L-\frac{\partial L}{\partial \dot{Q}} \dot{Q}\right) \epsilon t+\frac{\partial L}{\partial \dot{Q}} \frac{\epsilon Q}{2}$ and the dilatation operator

$$
D=-J=\frac{1}{2}\left(\dot{Q}^{2}+\frac{g}{Q^{2}}\right) t-\frac{1}{4}(\dot{Q} Q+Q \dot{Q}) .
$$


3. For special conformal transformations $t^{\prime}-t=\delta t=\epsilon t^{2}+O\left(\epsilon^{2}\right)$ and $\delta Q=$ $Q^{\prime}-Q=\epsilon t Q+O\left(\epsilon^{2}\right)$ in (C.4). From (C.7) we have $\Omega=-\frac{1}{2} \epsilon \frac{Q^{2}}{1-\epsilon t}$. The group parameters in this case are $\alpha=\delta=1, \beta=0, \gamma=-\epsilon$. The Noether current is $J \epsilon=\left(L-\frac{\partial L}{\partial \dot{Q}} \dot{Q}\right) \epsilon t^{2}+\frac{\partial L}{\partial \dot{Q}} \epsilon t Q-\frac{1}{2} \epsilon \frac{Q^{2}}{1-\epsilon t}$ and the generator of the special conformal transformations

$$
K=-J=\frac{1}{2}\left(\dot{Q}^{2}+\frac{g}{Q^{2}}\right) t^{2}-\frac{1}{2}(\dot{Q} Q+Q \dot{Q}) t+\frac{1}{2} Q^{2} .
$$

Since the operators must be Hermitean, one has to write the classical product $\dot{Q} Q$ as the symmetrized expression $\frac{1}{2}(\dot{Q} Q+Q \dot{Q})$. Note that the crucial term in $K$, namely $Q^{2}$, stems from the derivative $d \Omega / d t$ in the transformed Lagrangian (C.6).

One can check explicitly that the generators $H_{t}, D$ and $K$ obey the algebra of the generators of the conformal group

$$
[H, D]=i H, \quad[H, K]=2 i D, \quad[K, D]=-i K .
$$

To proof this, one has to use repeatedly the commutation relation $[Q, \dot{Q}]=i$, e.g.

$$
\begin{aligned}
{\left[\dot{Q}^{2}, \dot{Q} Q\right] } & =\dot{Q}^{3} Q-\dot{Q} Q \dot{Q}^{2}=\dot{Q}^{3} Q-\dot{Q}(i+\dot{Q} Q) \dot{Q} \\
& =\dot{Q}^{3} Q-i \dot{Q}^{2}-\dot{Q}^{2}(i+\dot{Q} Q)=-2 i \dot{Q}^{2} .
\end{aligned}
$$

A useful relation is $\dot{Q} Q^{-1}=Q^{-1} \dot{Q}+i Q^{-2}$ which can be proved by multiplying both sides by $Q$.

We can now examine the action of the generators of the conformal group on the state-space vectors

$$
\begin{aligned}
e^{-i H \epsilon}|\psi(t)\rangle & =|\psi(t+\epsilon)\rangle=|\psi(t)\rangle+\frac{d}{d t}|\psi(t)\rangle \epsilon+O\left(\epsilon^{2}\right), \\
e^{-i D \epsilon}|\psi(t)\rangle & =|\psi(t+t \epsilon)\rangle=|\psi(t)\rangle+\frac{d}{d t}|\psi(t)\rangle \epsilon t+O\left(\epsilon^{2}\right), \\
e^{-i K \epsilon}|\psi(t)\rangle & =\left|\psi\left(\frac{t}{1-\epsilon t}\right)\right\rangle=|\psi(t)\rangle+\frac{d}{d t}|\psi(t)\rangle \epsilon t^{2}+O\left(\epsilon^{2}\right) .
\end{aligned}
$$

Using the canonical commutation relation for the fields, $[Q, \dot{Q}]=i$, we can also 
obtain the evolution generated by the operators $H, D$ and $K$ in the Heisenberg picture

$$
\begin{aligned}
i[H, Q(t)] & =\frac{d Q(t)}{d t}, \\
i[D, Q(t)] & =t \frac{d Q(t)}{d t}-\frac{1}{2} Q(t), \\
i[K, Q(t)] & =t^{2} \frac{d Q(t)}{d t}-t Q(t) .
\end{aligned}
$$

In terms of the new time variable $\tau(3.19)$ and the new field $q(\tau)(3.22)$

$$
d \tau=\frac{d t}{u+v t+w t^{2}}, \quad q(\tau)=\frac{Q(t)}{\sqrt{u+v t+w t^{2}}},
$$

one obtains

$$
\begin{aligned}
A & =\frac{1}{2} \int d t\left(\dot{Q}^{2}-\frac{g}{Q^{2}}\right) \\
& =\frac{1}{2} \int d \tau\left(\dot{q}^{2}-\frac{g}{q^{2}}+\frac{v^{2}-4 u w}{4} q^{2}+\frac{1}{2} \frac{d}{d \tau}\left[(v+2 w t(\tau)) q^{2}\right]\right),
\end{aligned}
$$

Where we have used the identity

$$
(v+2 w t) q \dot{q}=\frac{1}{2} \partial_{\tau}\left[(v+2 w t) q^{2}\right]-\frac{1}{2} q^{2} \partial_{\tau}(v+2 w t(\tau)) .
$$

Thus, the transformed action differs from the original action only by a surface term which does not modify the equations of motion. 


\section{Appendix D}

\section{Useful Formulas for Higher Spin Equations in Anti-de Sitter Space}

\section{D.1 Arbitrary integer spin}

\section{D.1.1 The action in the local Lorentz frame}

Using the Christoffel symbols in A.2.2 one obtains from Eq. (A.58)

$$
\begin{aligned}
D_{M} \Phi_{N_{1} \cdots N_{J}}=\partial_{M} \Phi_{\{N\}}+\Omega(z) & \sum_{j}\left(\delta_{M}^{z} \Phi_{N_{j}, N_{1} \cdots N_{j-1} N_{j+1} \cdots N_{J}}+\right. \\
& \left.\delta_{N_{j}}^{z} \Phi_{M N_{1} \cdots N_{j-1} N_{j+1} \cdots N_{J}}+\eta_{M N_{j}} \Phi_{z N_{1} \cdots N_{j-1} N_{j+1} \cdots N_{J}}\right),
\end{aligned}
$$

with he warp factor $\Omega(z)=1 / z$ in AdS space.

The appearance of covariant derivatives (D.1) in the action for higher spin fields $(4.14,4.16)$ leads to multiple sums and quite complicated expressions. These, however simplify considerably if one goes to a local inertial frame with (Minkowskian) tangent indices. The transformation from general covariant indices to those with components in the local tangent space is achieved by the vielbeins, see (A.57)

$$
\hat{\Phi}_{A_{1} A_{2} \cdots A_{J}}=e_{A_{1}}^{N_{1}} e_{A_{2}}^{N_{2}} \cdots e_{A_{J}}^{N_{J}} \Phi_{N_{1} N_{2} \cdots N_{J}},
$$

and thus

$$
\hat{\Phi}_{N_{1} \ldots N_{J}}=\left(\frac{z}{R}\right)^{J} \Phi_{N_{1} \ldots N_{J}} .
$$

Notably, one can express the covariant derivatives in a general frame in terms of partial 
derivatives in a local tangent frame. One finds for the AdS metric

$$
D_{z} \Phi_{N_{1} \ldots N_{J}}=\left(\frac{R}{z}\right)^{J} \partial_{z} \hat{\Phi}_{N_{1} \ldots N_{J}}
$$

and

$$
\begin{aligned}
g^{\mu \mu^{\prime}} g^{\nu_{1} \nu_{1}^{\prime}} \ldots & g^{\nu_{J} \nu_{J}^{\prime}} D_{\mu} \Phi_{\nu_{1} \ldots \nu_{J}} D_{\mu^{\prime}} \Phi_{\nu_{1}^{\prime} \ldots \nu_{J}^{\prime}}= \\
& g^{\mu \mu^{\prime}} \eta^{\nu_{1} \nu_{1}^{\prime}} \ldots \eta^{\nu_{J} \nu_{J}^{\prime}}\left(\partial_{\mu} \hat{\Phi}_{\nu_{1} \ldots \nu_{J}} \partial_{\mu^{\prime}} \hat{\Phi}_{\nu_{1}^{\prime} \ldots \nu_{J}^{\prime}}+g^{z z} J \Omega^{2}(z) \hat{\Phi}_{\nu_{1} \ldots \nu_{J}} \hat{\Phi}_{\nu_{1}^{\prime} \ldots \nu_{J}^{\prime}}\right),
\end{aligned}
$$

where $\Omega(z)=1 / z$ is the AdS warp factor in the affine connection.

It is convenient for the application of the Euler-Lagrange equations $(4.17,4.18)$ to split action (4.16) into three terms:

1. A term $S_{\text {eff }}^{[0]}$ which contains only fields $\Phi_{\nu_{1} \ldots \nu_{J}}$ orthogonal to the holographic direction.

2. A term $S_{e f f}^{[1]}$, which is linear in the fields $\Phi_{z N_{2} \cdots N_{J}}^{*}, \Phi_{N_{1} z \cdots N_{J}}^{*}, \cdots, \Phi_{N_{1} N_{2} \cdots z}^{*}$.

3. The remainder, which is quadratic in fields with $z$-components, $i$. e., it contains terms such as $\Phi_{z N_{2} \ldots N_{J}}^{*} \Phi_{z N_{2}^{\prime} \ldots N_{J}^{\prime}}$. This last term does not contribute to the EulerLagrange equations (4.18), since, upon variation of the action, a vanishing term subject to the condition (4.15) is left.

Using (4.16), (D.4) and (D.5), and making use of the symmetry of the tensor fields, one finds,

$$
\begin{aligned}
S_{e f f}^{[0]}= & \int d^{d} x d z\left(\frac{R}{z}\right)^{d-1} e^{\varphi(z)} \eta^{\nu_{1} \nu_{1}^{\prime}} \cdots \eta^{\nu_{J} \nu_{J}^{\prime}}\left(-\partial_{z} \hat{\Phi}_{\nu_{1} \ldots \nu_{J}}^{*} \partial_{z} \hat{\Phi}_{\nu_{1}^{\prime} \ldots \nu_{J}^{\prime}}\right. \\
& \left.+\eta^{\mu \mu^{\prime}} \partial_{\mu} \hat{\Phi}_{\nu_{1} \ldots \nu_{J}}^{*} \partial_{\mu^{\prime}} \hat{\Phi}_{\nu_{1}^{\prime} \ldots \nu_{J}^{\prime}}-\left[\left(\frac{\mu_{e f f}(z) R}{z}\right)^{2}+J \Omega^{2}(z)\right] \hat{\Phi}_{\nu_{1} \ldots \nu_{J}}^{*} \hat{\Phi}_{\nu_{1}^{\prime} \ldots \nu_{J}^{\prime}}\right)
\end{aligned}
$$

and

$$
\begin{aligned}
S_{e f f}^{[1]}=\int d^{d} x d z\left(\frac{R}{z}\right)^{d-1} & e^{\varphi(z)}\left(-J \Omega(z) \eta^{\mu \mu^{\prime}} \eta^{N_{2} \nu_{2}^{\prime}} \cdots \eta^{N_{J} \nu_{J}^{\prime}} \partial_{\mu} \hat{\Phi}_{z N_{2} \ldots N_{J}}^{*} \hat{\Phi}_{\mu^{\prime} \nu_{2}^{\prime} \ldots \nu_{J}^{\prime}}\right. \\
& +J \Omega(z) \eta^{\mu \nu} \eta^{N_{2} \nu_{2}^{\prime}} \cdots \eta^{N_{J} \nu_{J}^{\prime}} \hat{\Phi}_{z N_{2} \ldots N_{J}}^{*} \partial_{\mu} \hat{\Phi}_{\nu \nu_{2}^{\prime} \ldots \nu_{J}^{\prime}} \\
& \left.-J(J-1) \Omega^{2}(z) \eta^{\mu \nu} \eta^{N_{3} \nu_{3}^{\prime}} \cdots \eta^{N_{J} \nu_{J}^{\prime}} \hat{\Phi}_{z z N_{3} \cdots N_{J}}^{*} \hat{\Phi}_{\mu \nu \nu_{3}^{\prime} \cdots \nu_{J}^{\prime}}\right) .
\end{aligned}
$$


As can be seen from the presence of the affine warp factor $\Omega(z)$ in (D.7), this term is only due to the affine connection and thus should only contribute to kinematical constraints.

From (D.6) one obtains, upon variation with respect to $\hat{\Phi}_{\nu_{1} \ldots \nu_{J}}^{*}(4.17)$, the equation of motion in the local tangent space

$$
\left[\partial_{\mu} \partial^{\mu}-\frac{z^{d-1}}{e^{\varphi(z)}} \partial_{z}\left(\frac{e^{\varphi(z)}}{z^{d-1}} \partial_{z}\right)+\frac{\left(\mu_{e f f}(z) R\right)^{2}+J}{z^{2}}\right] \hat{\Phi}_{\nu_{1} \ldots \nu_{J}}=0
$$

where $\partial_{\mu} \partial^{\mu} \equiv \eta^{\mu \nu} \partial_{\mu} \partial_{\nu}$. Using (D.7) one finds by variation with respect to $\hat{\Phi}_{N_{1} \cdots z \cdots N_{J}}^{*}$ (4.18) the kinematical constraints

$$
\eta^{\mu \nu} \partial_{\mu} \hat{\Phi}_{\nu \nu_{2} \cdots \nu_{J}}=0, \quad \eta^{\mu \nu} \hat{\Phi}_{\mu \nu \nu_{3} \cdots \nu_{J}}=0
$$

From (D.8) and (D.9) one obtains using (D.3) the wave equation in a general frame in terms of the original covariant tensor field $\Phi_{N_{1} \cdots N_{J}}$ given in $(4.19,4.20)$ and the kinematical constraints given by (4.21).

\section{D.1.2 Warped metric}

In the warped metric (4.26) the vielbein has the form

$$
\tilde{e}_{M}^{A}=\frac{R}{z} e^{\tilde{\varphi}(z)} \delta_{M}^{A}
$$

The Christoffel symbols for the warped metric (4.26) have the same form as (A.58)

$$
\Gamma_{M N}^{L}=-\tilde{\Omega}(z)\left(\delta_{M}^{5} \delta_{N}^{L}+\delta_{N}^{5} \delta_{M}^{L}-\eta^{L 5} \eta_{M N}\right)
$$

with the warp factor $\tilde{\Omega}(z)=1 / z-\partial_{z} \tilde{\varphi}(z)$.

The effective action, invariant with respect to the warped metric $\tilde{g}_{M N}$ is

$$
\tilde{S}_{e f f}=\int d^{d} x d z \sqrt{\tilde{g}} \tilde{g}^{\left\{N N^{\prime}\right\}}\left(\tilde{g}^{M M^{\prime}} D_{M} \Phi_{\{N\}}^{*} D_{M^{\prime}} \Phi_{\left\{N^{\prime}\right\}}-\tilde{\mu}_{e f f}^{2}(z) \Phi_{\{N\}}^{*} \Phi_{\left\{N^{\prime}\right\}}\right)
$$

where $\tilde{\mu}_{\text {eff }}(z)$ is again an effective mass.

Again one can go to a local tangent frame

$$
\hat{\Phi}_{A_{1} \cdots A_{J}}=e_{A_{1} \cdots A_{J}}^{N_{1} \cdots N_{J}} \Phi_{N_{1} \cdots N_{J}}=\left(\frac{z}{R}\right)^{J} e^{-J \tilde{\varphi}(z)} \Phi_{A_{1} \cdots A_{J}}
$$


and obtain

$$
\begin{aligned}
\tilde{S}_{\text {eff }}^{[0]}= & \int d^{d} x d z\left(\frac{R e^{\tilde{\varphi}(z)}}{z}\right)^{d-1} \eta^{\nu_{1} \nu_{1}^{\prime}} \cdots \eta^{\nu_{J} \nu_{J}^{\prime}}\left(-\partial_{z} \hat{\Phi}_{\{\nu\}}^{*} \partial_{z} \hat{\Phi}_{\left\{\nu^{\prime}\right\}}+\right. \\
& \left.\eta^{\mu \mu^{\prime}} \partial_{\mu} \hat{\Phi}_{\nu_{1} \cdot \nu_{J}}^{*} \partial_{\mu^{\prime}} \hat{\Phi}_{\nu_{1}^{\prime} \cdot \nu_{J}^{\prime}}-\left[\left(\frac{\mu_{e f f}(z) R e^{\tilde{\varphi}(z)}}{z}\right)^{2}+J \tilde{\Omega}^{2}(z)\right] \hat{\Phi}_{\nu_{1} \cdot \nu_{J}}^{*} \hat{\Phi}_{\nu_{1}^{\prime} \cdot \nu_{J}^{\prime}}\right) .
\end{aligned}
$$

Comparing (D.13) with the AdS action (D.6), one sees that both forms of the action are equivalent provided that one sets

$$
\tilde{\varphi}(z)=\frac{1}{d-1} \varphi(z) \quad \text { and } \quad\left(\tilde{\mu}_{e f f}(z) R\right)^{2} e^{2 \tilde{\varphi}}=\left(\mu_{e f f}(z) R\right)^{2}+\tilde{\Omega}^{2}(z)(J-1) .
$$

Also $\tilde{S}_{e f f}^{[1]}$ is equivalent to (D.7), only $\Omega$ is replaced by $\tilde{\Omega}$; since these warp factors factor out, their special form is not relevant for the kinematical conditions derived from (4.18). Therefore the kinematical constraints (4.21) follow also from the warped action (4.27).

\section{D.2 Arbitrary half integer spin}

\section{D.2.1 General treatment}

The covariant derivative of a Rarita-Schwinger spinor $\Psi_{\{N\}}$ is given by

$$
D_{M} \Psi_{N_{1} \cdots N_{T}}=\partial_{M} \Psi_{N_{1} \cdots N_{T}}-\frac{i}{2} \omega_{M}^{A B} \Sigma_{A B} \Psi_{N_{1} \cdots N_{T}}-\sum_{j} \Gamma_{M N_{j}}^{L} \Psi_{N_{1} N_{j-1} L N_{j=1} \cdots N_{T}}
$$

where $\Sigma_{A B}$ are the generators of the Lorentz group in the spinor representation

$$
\Sigma_{A B}=\frac{i}{4}\left[\Gamma_{A}, \Gamma_{B}\right]
$$

and the tangent space Dirac matrices obey the usual anti-commutation relation

$$
\Gamma^{A} \Gamma^{B}+\Gamma^{B} \Gamma^{A}=2 \eta^{A B}
$$

The spin connection in AdS is

$$
w_{M}^{A B}=\Omega(z)\left(\eta^{A z} \delta_{M}^{B}-\eta^{B z} \delta_{M}^{A}\right)
$$


with $\Omega(z)=1 / z$.

For even $d$ one can choose the set of gamma matrices $\Gamma^{A}=\left(\Gamma^{\mu}, \Gamma^{z}\right)$ with $\Gamma^{z}=$ $\Gamma^{0} \Gamma^{1} \cdots \Gamma^{d-1}$. For $d=4$ one has

$$
\Gamma^{\mu}=\gamma^{\mu}, \quad \Gamma^{z}=-\Gamma_{z}=-i \gamma^{5}
$$

where $\gamma^{\mu}$ and $\gamma^{5}$ are the usual 4-dimensional Dirac matrices with $\gamma^{5} \equiv i \gamma^{0} \gamma^{1} \gamma^{2} \gamma^{3}$ and $\left(\gamma^{5}\right)^{2}=+1$. The spin connections are given by

$$
\omega_{\mu}^{z \alpha}=-\omega_{\mu}^{\alpha z}=\Omega(z) \delta_{\mu}^{\alpha}
$$

all other components $\omega_{M}^{A B}$ are zero.

The covariant derivatives of a Rarita-Schwinger spinor in AdS are

$$
\begin{aligned}
D_{z} \Psi_{N_{1} \cdots N_{T}}= & \partial_{z} \Psi_{N_{1} \cdots N_{T}}+T \Omega(z) \Psi_{N_{1} \cdots N_{T}}, \\
D_{\mu} \Psi_{N_{1} \cdots N_{T}}= & \partial_{\mu} \Psi_{N_{1} \cdots N_{T}}+\frac{1}{2} \Omega(z) \Gamma_{\mu} \Gamma_{z} \Psi_{N_{1} \cdots N_{T}}+ \\
& \Omega(z) \sum_{j}\left(\delta_{N_{j}}^{z} \Psi_{N_{1} N_{j-1}, \mu, N_{j+1}, \cdots N_{T}}+\eta_{\mu N_{j}} \Psi_{N_{1} N_{j-1}, z, N_{j+1}, \cdots N_{T}}\right) .
\end{aligned}
$$

where, as usual the index $z$ denotes the $(d+1)$ holographic direction.

The derivation of the equation of motion follows the lines outlined in Secs. 4.2 and D.1. One introduces fields with tangent indices using a local Lorentz frame as in Sec. D.1.1

$$
\hat{\Psi}_{A_{1} \ldots A_{T}}=e_{A_{1}}^{N_{1}} \cdots e_{A_{T}}^{N_{T}} \Psi_{N_{1} \ldots N_{T}}=\left(\frac{z}{R}\right)^{T} \Psi_{A_{1} \ldots A_{T}},
$$

and separate the action into a part $S_{F e f f}^{[0]}$ containing only spinors orthogonal to the holographic direction, and a term $S_{F e f f}^{[1]}$, containing terms linear in $\bar{\Psi}_{z N_{2} \ldots N_{T}}$; the remainder does not contribute to the Euler-Lagrange equations (4.42). Since the fermion action is linear in the derivatives, the calculations are considerably simpler than for the integer spin. One obtains from (D.21)

$$
\begin{array}{rl}
S_{F \text { eff }}^{[0]}=\int d^{d} x d & z\left(\frac{R}{z}\right)^{d+1} e^{\varphi(z)} \eta^{\nu_{1} \nu_{1}^{\prime}} \ldots \eta^{\nu_{T} \nu_{T}^{\prime}}\left(\frac{i}{2} e_{A}^{M} \overline{\hat{\Psi}}_{\nu_{1} \cdots \nu_{T}} \Gamma^{A} \partial_{M} \hat{\Psi}_{\nu_{1}^{\prime} \ldots \nu_{T}^{\prime}}\right. \\
& \left.-\frac{i}{2} e_{A}^{M}\left(\partial_{M} \overline{\hat{\Psi}}_{\nu_{1} \ldots \nu_{T}}\right) \Gamma^{A} \hat{\Psi}_{\nu_{1}^{\prime} \cdots \nu_{T}^{\prime}}-(\mu+\rho(z)) \overline{\hat{\Psi}}_{\nu_{1} \cdots \nu_{T}} \hat{\Psi}_{\nu_{1}^{\prime} \cdots \nu_{T}^{\prime}}\right)
\end{array}
$$


and

$$
\begin{aligned}
S_{F e f f}^{[1]}=-\int d^{d} x d z\left(\frac{R}{z}\right)^{d} e^{\varphi(z)} \eta^{N_{2} N_{2}^{\prime}} \cdots \eta^{N_{T} N_{T}^{\prime}} & \\
& T \Omega(z)\left(\overline{\hat{\Psi}}_{z N_{2} \cdots N_{T}} \Gamma^{\mu} \hat{\Psi}_{\mu N_{2}^{\prime} \cdots N_{T}^{\prime}}+\overline{\hat{\Psi}}_{\mu N_{2} \cdots N_{T}} \Gamma^{\mu} \hat{\Psi}_{z N_{2}^{\prime} \ldots N_{T}^{\prime}}\right),
\end{aligned}
$$

where the factor of the affine connection $\Omega(z)=1 / z$ follows from Eqs. (A.58) and (D.18). Performing a partial integration, the action (D.24) becomes:

$$
\begin{array}{rl}
S_{F \text { eff }}^{[0]}=\int d^{d} x & d z\left(\frac{R}{z}\right)^{d} e^{\varphi(z)} \eta^{\nu_{1} \nu_{1}^{\prime}} \cdots \eta^{\nu_{T} \nu_{T}^{\prime}} \\
& \hat{\hat{\Psi}}_{\nu_{1} \cdots \nu_{T}}\left(i \eta^{N M} \Gamma_{M} \partial_{N}+\frac{i}{2 z} \Gamma_{z}\left(d-z \varphi^{\prime}(z)\right)-\mu R-\rho(z)\right) \hat{\Psi}_{\nu_{1}^{\prime} \ldots \nu_{T}^{\prime}}
\end{array}
$$

plus surface terms.

The variation of (D.25) yields indeed the Rarita-Schwinger condition in physical space-time (4.37)

$$
\gamma^{\nu} \hat{\Psi}_{\nu \nu_{2} \ldots \nu_{T}}=0
$$

and the variation of (D.26) provides the AdS Dirac-like wave equation

$$
\left[i\left(z \eta^{M N} \Gamma_{M} \partial_{N}+\frac{d-z \varphi^{\prime}}{2} \Gamma_{z}\right)-\mu R-R \rho(z)\right] \hat{\Psi}_{\nu_{1} \ldots \nu_{T}}=0 .
$$

Going back to covariant Rarita-Schwinger spinors $\Psi_{\nu \nu_{2} \ldots \nu_{T}}$ one obtains immediately (4.43) and (4.44).

\section{D.2.2 Spin- $\frac{3}{2}$ Rarita-Schwinger field in AdS space}

The generalization $[183,184]$ of the Rarita-Schwinger action [179] to $\mathrm{AdS}_{d+1}$ is

$$
S=\int d^{d} x d z \sqrt{g} \bar{\Psi}_{N}\left(i \tilde{\Gamma}^{\left[N M N^{\prime}\right]} D_{M}-\mu \tilde{\Gamma}^{\left[N N^{\prime}\right]}\right) \Psi_{N}
$$

where $\tilde{\Gamma}^{\left[N M N^{\prime}\right]}$ and $\tilde{\Gamma}^{\left[N N^{\prime}\right]}$ are the antisymmetrical products of three and two Dirac matrices $\tilde{\Gamma}^{M}=e_{A}^{M} \Gamma^{A}=\frac{z}{R} \delta_{A}^{M} \Gamma^{A}$, with tangent space matrices $\Gamma^{A}$ given by (D.17). From the variation of this action one obtains the generalization of the Rarita-Schwinger equation

$$
\left(i \tilde{\Gamma}^{\left[N M N^{\prime}\right]} D_{M}-\mu \tilde{\Gamma}^{\left[N N^{\prime}\right]}\right) \Psi_{N^{\prime}}=0
$$


The Christoffel symbols in the covariant derivative can be omitted due to the the antisymmetry of the indices in $\tilde{\Gamma}^{\left[N M N^{\prime}\right]}$ and only the spin connection must be taken into account. Eq. (D.30) leads to the Rarita-Schwinger condition [183]

$$
\Gamma^{M} \Psi_{M}=0
$$

and the generalized Dirac equation [184]

$$
\left[i\left(z \eta^{M N} \Gamma_{M} \partial_{N}+\frac{d}{2} \Gamma_{z}\right)-\mu R\right] \hat{\Psi}_{A}=\Gamma_{A} \hat{\Psi}_{z}
$$

for the spinor with tangent indices $\hat{\Psi}_{A}=\frac{z}{R} \delta_{A}^{M} \Psi_{M}$. These equations agree for $T=$ 1, $\varphi(z)=\rho(z)=0$ and $\hat{\Psi}_{z}=0$ with Eq. (D.28), derived from the effective action (4.39), for $\varphi=\rho=0$. 


\section{Appendix E}

\section{Light-Front Holographic Mapping and the Energy-Momentum Tensor}

\section{E.1 Gravitational form factor of composite hadrons}

In Chapter 6 we described the matching of the electromagnetic current matrix elements in AdS space with the corresponding expression derived from light-front quantization in physical space-time, thus establishing a precise relation between wave functions in AdS space and the light-front wave functions describing the internal structure of hadrons. One may ask if the holographic mapping found in [75] for the electromagnetic current is specific to the charge distribution within a hadron or a general feature of light-front holographic QCD. In fact, the matrix elements of local operators of hadronic composite systems, such as currents, angular momentum and the energy-momentum tensor, have exact Lorentz-invariant representations in the light front in terms of the overlap of light-front wave functions and thus the LF holographic mapping is a general property of LF observables. In this appendix we show explicitly that one obtains indeed identical holographic mapping using matrix elements of the energy-momentum tensor [81, 82]. 


\section{E.1.1 Meson gravitational form factor in AdS space}

The action for gravity coupled to a scalar field in $\mathrm{AdS}_{5}$ is

$$
\begin{aligned}
S & =\frac{1}{\kappa^{2}} \int d^{4} x d z \sqrt{g}(\mathcal{R}-2 \Lambda)+S_{M} \\
& =S_{G}+S_{M},
\end{aligned}
$$

where $g \equiv\left|\operatorname{det} g_{M N}\right|$ and $\mathcal{R}$ is the scalar curvature (See A.1.2), $\kappa$ is the 5 -dimensional Newton constant, $g$ is the determinant of the metric tensor, and $\Lambda$ is a bulk 'cosmological' constant. The first term in the action $S_{G}$ describes the dynamics of the gravitational fields $g_{M N}$ and determines the background, which is AdS space (See A.2.2). The coordinates of $\mathrm{AdS}_{5}$ are the Minkowski coordinates $x^{\mu}$ and $z$ labeled $x^{M}=\left(x^{\mu}, z\right)$, with $M, N=0, \cdots 4$. The dynamics of all other fields, the matter fields, is included in $S_{M}$. To simplify the discussion we consider a scalar field in AdS. In this case the matter content is represented by the AdS action:

$$
S_{M}=\int d^{4} x d z \sqrt{g}\left(g^{M N} \partial_{M} \Phi^{*} \partial_{N} \Phi-\mu^{2} \Phi^{*} \Phi\right),
$$

which describes a meson in AdS space. The symmetric and gauge-invariant Hilbert energy-momentum tensor of the mater fields follows from the functional derivative

$$
\Theta_{M N}\left(x^{L}\right)=-\frac{2}{\sqrt{g}} \frac{\delta S_{M}}{\delta g^{M N}\left(x^{L}\right)},
$$

and is given by

$$
\Theta_{M N}=\partial_{M} \Phi^{*} \partial_{N} \Phi+\partial_{N} \Phi^{*} \partial_{M} \Phi-g_{M N}\left(\partial^{L} \Phi^{*} \partial_{L} \Phi-\mu^{2} \Phi^{*} \Phi\right) .
$$

To determine the matrix elements of the energy-momentum tensor for arbitrary momentum transfer, we must first identify the interaction term in the action of the matter fields with an external gravitational source at the AdS boundary [82]. To this end we consider a small deformation of the metric about its AdS background: $\bar{g}_{M N}=$ $g_{M N}+h_{M N}$, and expand expand $S_{M}$ to first order in $h_{M N}$

$$
S_{M}\left[h_{M N}\right]=S_{M}[0]+\frac{1}{2} \int d^{4} x d z \sqrt{g} h_{M N} \Theta^{M N}+\mathcal{O}\left(h^{2}\right),
$$

where we have used the relation $\Theta^{M N} \delta g_{M N}=-\Theta_{M N} \delta g^{M N}$ which follows from $g^{M N} \delta g_{M N}=$ 
$-g^{M N} \delta g_{M N}$. Thus, in the weak gravitational approximation the coupling of an external graviton field $h_{M N}$ to matter is given by the interaction term

$$
S_{I}=\frac{1}{2} \int d^{4} x d z \sqrt{g} h_{M N} \Theta^{M N}
$$

Likewise, we can determine the AdS equation of motion of the graviton field $h_{M N}$ by substituting the modified metric $\bar{g}_{M N}=g_{M N}+h_{M N}$ into the gravitational action $S_{G}$. We find

$$
S_{G}\left[h_{M N}\right]=S_{G}[0]+\frac{1}{4 \kappa^{2}} \int d^{4} x d z \sqrt{g}\left(\partial_{N} h^{L M} \partial^{N} h_{L M}-\frac{1}{2} \partial_{L} h \partial^{L} h\right)+\mathcal{O}\left(h^{2}\right),
$$

where the trace $h_{L}^{L}$ is denoted by $h$. In deriving (E.7) we have made use of the gauge invariance of the theory $h_{L M}^{\prime}=h_{L M}+\partial_{L} \epsilon_{M}+\partial_{M} \epsilon_{L}$ to impose the harmonic gauge condition $\partial_{\ell} h_{m}^{\ell}=\frac{1}{2} \partial_{m} h$. The action describing the dynamical fields $h_{L M}$ is given by the linearized form

$$
S_{h}=\frac{1}{4 \kappa^{2}} \int d^{4} x d z \sqrt{g}\left(\partial_{N} h^{L M} \partial^{N} h_{L M}-\frac{1}{2} \partial_{L} h \partial^{L} h\right)
$$

resembling the treatment of an ordinary gauge field. The total bulk action describing the coupling of gravity and matter with an external graviton in the weak field approximation thus has two additional terms: $S=S_{G}+S_{M}+S_{h}+S_{I}$.

\section{Hadronic transition matrix elements in AdS and gravitational form factor}

To simplify the discussion, we consider the holographic mapping of matrix elements of the energy-momentum tensor of mesons, where only one gravitational form factor is present, but the results can be extended to other hadrons. We also describe the bulk AdS geometry with a model with a wall at a finite distance $z=1 / \Lambda_{\mathrm{QCD}}$ - the hard wall model of Ref. [55]. In the higher-dimensional background theory the matrix element of the energy-momentum tensor for the hadronic transition $P \rightarrow P^{\prime}$, follows from the interaction term (E.6) describing the coupling of the meson field $\Phi_{P}(x, z)$ with the external graviton field $h_{M N}(x, z)$ propagating in AdS space,

$$
\int d^{4} x d z \sqrt{g} h_{M N}\left(\partial^{M} \Phi_{P^{\prime}}^{*} \partial^{N} \Phi_{P}+\partial^{N} \Phi_{P^{\prime}}^{*} \partial^{M} \Phi_{P}\right)
$$


where we have dropped the second term in (E.4), which vanishes on-shell modulo a surface term. The hadronic transition matrix element has the form

$$
\begin{array}{r}
\int d^{4} x d z \sqrt{g} h_{M N}(x, z)\left(\partial^{M} \Phi_{P^{\prime}}^{*}(x, z) \partial^{N} \Phi_{P}(x, z)+\partial^{N} \Phi_{P^{\prime}}^{*}(x, z) \partial^{M} \Phi_{P}(x, z)\right) \\
\sim(2 \pi)^{4} \delta^{4}\left(P^{\prime}-P-q\right) \epsilon_{\nu}^{\mu}\left(P^{\nu} P_{\mu}^{\prime}+P_{\mu} P^{\prime \nu}\right) A\left(q^{2}\right)
\end{array}
$$

where the meson has initial and final four momentum $P$ and $P^{\prime}$ respectively and $q$ is the four-momentum transferred to the pion by the graviton with polarization $\epsilon_{\nu}^{\mu}$. The expression on the right-hand side of (E.10) represents the space-like gravitational form factor in physical space-time:

$$
\left\langle P^{\prime}\left|\Theta_{\mu}^{\nu}\right| P\right\rangle=\left(P^{\nu} P_{\mu}^{\prime}+P_{\mu} P^{\prime \nu}\right) A\left(q^{2}\right)
$$

It is the matrix element of the energy-momentum tensor operator in QCD $\Theta_{\mu \nu}$ obtained below in Sec. E.1.2, and represents a local coupling to pointlike constituents. Despite the fact that the expressions for the transition amplitudes are quite different, one can show that in the semiclassical approximation, discussed in Chapters 2 and 6, a precise mapping can be carried out at fixed light-front time for an arbitrary number of partons in the bound state [81].

The propagation of the meson in AdS space is described by a normalizable mode $\Phi_{P}\left(x^{\mu}, z\right)=e^{i P \cdot x} \Phi(z)$ with invariant mass $P_{\mu} P^{\mu}=M^{2}$ and plane waves along the physical coordinates $x^{\mu}$. The boundary limit of the graviton probe is a plane wave along the Minkowski coordinates with polarization indices along physical space-time according to $h_{\mu}^{\nu}(x, z \rightarrow 0)=\epsilon_{\mu}^{\nu}(q) e^{i q \cdot x}$. We thus write

$$
h_{\mu}^{\nu}(x, z)=\epsilon_{\mu}^{\nu}(q) e^{i q \cdot x} H\left(q^{2}, z\right)
$$

with

$$
H\left(q^{2}=0, z\right)=H\left(q^{2}, z=0\right)=1
$$

Extracting the overall factor $(2 \pi)^{4} \delta^{4}\left(P^{\prime}-P-q\right)$ from momentum conservation at the vertex, which arises from integration over Minkowski variables in (E.10), we find [82]

$$
A\left(Q^{2}\right)=R^{3} \int^{1 / \Lambda_{\mathrm{QCD}}} \frac{d z}{z^{3}} H\left(q^{2}, z\right) \Phi^{2}(z)
$$

with $A(0)=1$. The gravitational form factor in AdS is thus represented as the $z$ - 
overlap of the normalizable modes dual to the incoming and outgoing hadrons, $\Phi_{P}$ and $\Phi_{P^{\prime}}$, with the non-normalizable mode, $H\left(q^{2}, z\right)$, dual to the external graviton source [82]; this provides the form of the gravitational transition matrix element analogous to the electromagnetic form factor in AdS [77]. It is interesting to notice that in holographic QCD hadrons appear noticeably more compact measured by the gravitational form factor than by the corresponding charge form factor [82, 180, 369].

\section{E.1.2 Meson gravitational form factor in light-front QCD}

The symmetric and gauge-invariant expression for the energy-momentum tensor $\Theta_{\mu \nu}$ is obtained by varying the QCD action with respect to the four-dimensional Minkowski space-time metric $\mathrm{g}_{\mu \nu}(x)$

$$
\Theta_{\mu \nu}\left(x^{\rho}\right)=-\frac{2}{\sqrt{\mathrm{g}}} \frac{\delta S_{\mathrm{QCD}}}{\delta \mathrm{g}^{\mu \nu}\left(x^{\rho}\right)},
$$

where $S_{\mathrm{QCD}}=\int d^{4} x \sqrt{\mathrm{g}} \mathcal{L}_{\mathrm{QCD}}$ and $\mathrm{g} \equiv\left|\operatorname{det} \mathrm{g}_{\mu \nu}\right|$. From (2.9) we obtain he result:

$$
\Theta_{\mu \nu}=\frac{1}{2} \bar{\psi} i\left(\gamma_{\mu} D_{\nu}+\gamma_{\nu} D_{\mu}\right) \psi-\mathrm{g}_{\mu \nu} \psi(i \not D-m) \psi-G_{\mu \lambda}^{a} G_{\nu}^{a \lambda}+\frac{1}{4} \mathrm{~g}_{\mu \nu} G_{\lambda \sigma}^{a} G^{a \lambda \sigma}
$$

where the first two terms correspond to the fermionic contribution to the energy-momentum tensor and the last two to the gluonic contribution.

In the front form, the gravitational form factor is conveniently computed from the matrix elements of the plus-plus component of the energy momentum tensor at LF time $x^{+}=0$ :

$$
\left\langle P^{\prime}\left|\Theta^{++}(0)\right| P\right\rangle=2\left(P^{+}\right)^{2} A\left(q^{2}\right),
$$

where $P^{\prime}=P+q$. In the LF gauge $A^{+}=0$ the fermionic component of the operator $\Theta^{++}$is

$$
\Theta^{++}(x)=\frac{i}{2} \sum_{f} \bar{\psi}_{f}(x) \gamma^{+} \overleftrightarrow{\partial}+\psi_{f}(x)
$$

where an integration by parts is carried out to write $\Theta^{++}$in its hermitian operator form. The sum in (E.18) extends over all the types of quarks $f$ present in the hadron ${ }^{1}$. The expression for the operator $\Theta^{++}(0)$ in the particle number representation follows from

\footnotetext{
${ }^{1}$ The plus-plus component of the energy-momentum does not connect Fock states with different numbers of constituents in the $q^{+}=0$ frame [78]. In the semiclassical AdS/CFT correspondence there are no quantum effects, and only the valence Fock state contributes to the hadronic wave function. In this approximation we need to consider only the quark contribution to the energy momentum tensor in (E.18). Notice also that the second term of the energy-momentum tensor (E.16) does not appear in the expression for $\Theta^{++}$since the metric component $\mathrm{g}^{++}$is zero in the light-front (Appendix B).
} 
the momentum expansion of the Dirac field $\psi(x)$ in terms of creation and annihilation operators given by (2.14). The matrix element the operator $\Theta^{++}$is then computed by expanding the initial and final meson states $\left|\psi_{M}\left(P^{+}, \mathbf{P}_{\perp}\right)\right\rangle$ in terms of its Fock components (2.23). Using the normalization condition (2.22) for each individual constituent, and after integration over the intermediate variables in the $q^{+}=0$ frame we find the expression for the gravitational form factor of a meson $[160,81]$

$$
A_{M}\left(q^{2}\right)=\sum_{n} \int\left[d x_{i}\right]\left[d^{2} \mathbf{k}_{\perp i}\right] \sum_{f=1}^{n} x_{f} \psi_{n / M}^{*}\left(x_{i}, \mathbf{k}_{\perp i}^{\prime}, \lambda_{i}\right) \psi_{n / M}\left(x_{i}, \mathbf{k}_{\perp i}, \lambda_{i}\right)
$$

where the sum is over all the partons in each Fock state $n$. The variables of the lightcone Fock components in the final state are given by $\mathbf{k}_{\perp i}^{\prime}=\mathbf{k}_{\perp i}+\left(1-x_{i}\right) \mathbf{q}_{\perp}$ for a struck constituent quark and $\mathbf{k}_{\perp i}^{\prime}=\mathbf{k}_{\perp i}-x_{i} \mathbf{q}_{\perp}$ for each spectator. Notice that each type of parton contributes to the gravitational form factor with struck constituent lightcone momentum fractions $x_{f}$, instead of the electromagnetic constituent charge $e_{f}$ which appears in the electromagnetic form factor. Since the longitudinal momentum fractions of the constituents add to one, $\sum_{f} x_{f}=1$, the momentum sum rule is satisfied at $q=0$ : $A(0)=1$. The formula is exact if the sum is over all Fock states $n$.

In order to compare with AdS results it is convenient to express the LF expressions in the transverse impact representation since the bilinear forms may be expressed in terms of the product of light-front wave functions with identical variables. We substitute (2.35) in the formula (E.19). Integration over $k_{\perp}$ phase space gives us $n-1$ delta functions to integrate over the $n-1$ intermediate transverse variables with the result [81]

$$
A_{M}\left(q^{2}\right)=\sum_{n} \prod_{j=1}^{n-1} \int d x_{j} d^{2} \mathbf{b}_{\perp j} \sum_{f=1}^{n} x_{f} \exp \left(i \mathbf{q}_{\perp} \cdot \sum_{k=1}^{n-1} x_{k} \mathbf{b}_{\perp k}\right)\left|\psi_{n / M}\left(x_{j}, \mathbf{b}_{\perp j}, \lambda_{j}\right)\right|^{2}
$$

corresponding to a change of transverse momentum $x_{j} \mathbf{q}_{\perp}$ for each of the $n-1$ spectators.

For a baryon, the spin-conserving form factor $A\left(q^{2}\right)$ is the analog of the Dirac form factor $F_{1}\left(q^{2}\right)$. It allows one to measure the momentum fraction carried by each constituent. There is also a spin-flip form factor $B\left(q^{2}\right)$, the analog of the Pauli form factor $F_{2}\left(Q^{2}\right)$ of a nucleon, which provides a measure of the orbital angular momentum carried by each quark and gluon constituent of a hadron at $q^{2}=0$. An important constraint is $B(0)=0$, the vanishing of the anomalous gravitomagnetic moment of fermions [370, 371]. For a composite bound state this means that the anomalous gravitomagnetic moment of a hadron vanishes when summed over all the constituents. The explicit verification of 
these relations, Fock state by Fock state, can be obtained in the light-front quantization of QCD in light-cone gauge [160]. Physically $B(0)=0$ corresponds to the fact that the sum of the $n$ orbital angular momenta $L$ in an $n$-parton Fock state must vanish since there are only $n-1$ independent orbital angular momenta.

\section{E.1.3 Light-front holographic mapping}

The mapping of AdS transition amplitudes to light-front QCD transition matrix elements is much simplified for two-parton hadronic states. The light-front expression for a meson form factor in impact space is given by (E.20) and includes the contribution of each struck parton with longitudinal momentum fraction $x_{f}$. For $n=2$, there are two terms which contribute to the $f$-sum in (E.20). Exchanging $x \leftrightarrow 1-x$ in the second term and integrating over angles we find

$$
A_{\pi}\left(Q^{2}\right)=4 \pi \int_{0}^{1} \frac{d x}{(1-x)} \int \zeta d \zeta J_{0}\left(\zeta q \sqrt{\frac{1-x}{x}}\right)\left|\psi_{q \bar{q} / M}(x, \zeta)\right|^{2}
$$

where $\zeta^{2}=x(1-x) \mathbf{b}_{\perp}^{2}$ and $A_{M}(0)=1$.

We now consider the expression for the hadronic gravitational form factor in AdS space (E.14). Since the energy momentum tensor $\Theta^{M N}$ is gauge invariant, we may impose a more restricted gauge condition in order to simplify the calculations and use the general covariance of the theory to obtain the final result. We choose the harmonic-traceless gauge $\partial_{L} h_{M}^{L}=\frac{1}{2} \partial_{M} h=0$ and we consider the propagation inside AdS space of a graviton probe $h_{M N}$ with vanishing metric components along the $z$-coordinate $h_{z z}=h_{z \mu}=0$. The set of linearized Einstein equations from (E.8) reduce for $H\left(Q^{2}, z\right)$ in (E.12) to the wave equation [82]

$$
\left[\frac{d^{2}}{d z^{2}}-\frac{3}{z} \frac{d}{d z}-Q^{2}\right] H\left(Q^{2}, z\right)=0
$$

which describes the propagation of the external graviton inside AdS space. Its solution subject to the boundary conditions (E.13) is $\left(Q^{2}=-q^{2}>0\right)$

$$
H\left(Q^{2}, z\right)=\frac{1}{2} Q^{2} z^{2} K_{2}(z Q)
$$

the result obtained by Abidin and Carlson [82]. Using the integral representation of 
$H\left(Q^{2}, z\right)$ from $(\mathrm{G} .17)$

$$
H\left(Q^{2}, z\right)=2 \int_{0}^{1} x d x J_{0}\left(z Q \sqrt{\frac{1-x}{x}}\right)
$$

the AdS gravitational form factor (E.14) can be expressed as

$$
A\left(Q^{2}\right)=2 R^{3} \int_{0}^{1} x d x \int \frac{d z}{z^{3}} J_{0}\left(z Q \sqrt{\frac{1-x}{x}}\right)\left|\Phi_{\pi}(z)\right|^{2} .
$$

We now compare with the light-front QCD gravitational form factor (E.21) using the expression of the light-front wave function (2.40)

$$
\psi(x, \zeta, \varphi)=e^{i L \varphi} X(x) \frac{\phi(\zeta)}{\sqrt{2 \pi \zeta}}
$$

which we use to factor out the longitudinal and transverse modes $\phi(\zeta)$ and $X(x)$ from the LFWF in (E.21). Both expressions for the gravitational form factor are identical for arbitrary values of $Q$ provided that [81]

$$
\phi(\zeta)=\left(\frac{R}{\zeta}\right)^{-3 / 2} \Phi(\zeta) \quad \text { and } \quad X(x)=\sqrt{x(1-x)}
$$

This comparison allows us to identify the transverse impact LF variable $\zeta$ with the holographic variable $z, z \rightarrow \zeta=\sqrt{x(1-x)}\left|\mathbf{b}_{\perp}\right|^{2}$. The results are identical to those obtained from the mapping of the electromagnetic form factor in Sec. 6.1.3.

\footnotetext{
${ }^{2}$ Extension of the results to arbitrary $n$ follows from the $x$-weighted definition of the transverse impact variable of the $n-1$ spectator system given by Eq. (2.47).
} 


\section{Appendix F}

\section{Propagators in the Limiting Theory of $\mathrm{AdS}_{5}$}

\section{F.1 AdS boundary conditions and gauge/gravity cor- respondence}

The formal statement of the duality between a gravity theory on a $(d+1)$-dimensional Anti-de Sitter $A d S_{d+1}$ space and the strong coupling limit of a conformal field theory (CFT) on the $d$-dimensional flat space boundary at $z=0$, is expressed in terms of the $d+1$ partition function for a field $\Phi(x, z)$ propagating in the bulk

$$
Z_{\text {grav }}[\Phi]=\int \mathcal{D}[\Phi] e^{i S_{\text {grav }}[\Phi]}
$$

and the $d$-dimensional generating functional of correlation functions of the boundary conformal theory in presence of an external source $j(x)$ :

$$
Z_{C F T}[j]=\left\langle\exp \left(i \int d^{d} x j(x) \mathcal{O}(x)\right)\right\rangle,
$$

where $\mathcal{O}$ is a local interpolating operator. The interpolating operators $\mathcal{O}$ of the boundary quantum field theory are constructed from local products of fields and their covariant derivatives, taken at the same point in four-dimensional space-time.

The correlation function $\left\langle\mathcal{O}\left(x_{1}\right) \cdots \mathcal{O}\left(x_{n}\right)\right\rangle$ follows from the functional derivatives of 
the generating functional of the connected Green functions $\log Z_{C F T}[j]$ :

$$
\left\langle\mathcal{O}\left(x_{1}\right) \cdots \mathcal{O}\left(x_{n}\right)\right\rangle=(-i)^{n} \frac{\delta}{\delta j\left(x_{1}\right)} \cdots \frac{\delta}{\delta j\left(x_{n}\right)} \log Z_{C F T}[j]
$$

As a specific example consider a scalar field in AdS. In the boundary limit $z \rightarrow 0$, the independent solutions behave as

$$
\Phi(x, z) \rightarrow z^{\Delta} \Phi_{+}(x)+z^{d-\Delta} \Phi_{-}(x)
$$

where $\Delta$ is the scaling dimension. The non-normalizable solution $\Phi_{-}$has the leading boundary behavior and is the boundary value of the bulk field $\Phi$ which couples to a QCD interpolating operator:

$$
\lim _{z \rightarrow 0} z^{\Delta-d} \Phi(x, z)=j(x)
$$

where $j(x)=\Phi_{-}(x)$, a finite quantity. The normalizable solution $\Phi_{+}$is the response function and corresponds to the physical states [372].

The precise relation of the gravity theory on AdS space to the conformal field theory at its boundary is $[27,28]$

$$
Z_{C F T}[j]=Z_{\text {grav }}\left[\Phi_{[z=\epsilon]} \rightarrow j\right]
$$

where the partition function (F.1) on $A d S_{d+1}$ is integrated over all possible configurations $\Phi$ in the bulk which approach its boundary value $j$. In the classical limit we neglect the contributions from quantum fluctuations to the gravity partition function, then the generating functional of the four-dimensional gauge theory $\log Z[j]$ (F.2) is precisely equal to the classical (on-shell) gravity action $S_{\text {grav }}^{\text {on-shell }}\left[\Phi_{[z=\epsilon]}^{\mathrm{cl}} \rightarrow j\right]$ :

$$
\log Z[j]=i S_{\text {grav }}^{\mathrm{on}-\mathrm{shell}}\left[\Phi_{[z=\epsilon]}^{\mathrm{cl}} \rightarrow j\right]
$$

evaluated in terms of the classical solution $\Phi^{\mathrm{cl}}$ to the bulk equation of motion. This defines the semiclassical approximation to the conformal field theory. In the bottom-up phenomenological approach, the effective action in the bulk is usually modified for large values of $z$ to incorporate confinement and is truncated at the quadratic level. 
Consider the AdS action for the scalar field $\Phi^{1}$

$$
S_{\text {grav }}=\frac{1}{2} \int d^{d} x d z \sqrt{g}\left(g^{M N} \partial_{M} \Phi \partial_{N} \Phi-\mu^{2} \Phi^{2}\right)
$$

where $g=\left(\frac{R}{z}\right)^{2 d+2}$. Integrating by parts, and using the equation of motion, the bulk contribution to the action vanishes, and one is left with a non-vanishing surface term in the boundary

$$
S_{\text {grav }}^{\text {on-shell }}=-\frac{1}{2} \int d^{d} x\left(\sqrt{g} \Phi g^{z z} \partial_{z} \Phi\right)_{z=\epsilon} \cdot
$$

We can compute the expectation value of $\mathcal{O}$

$$
\langle\mathcal{O}(x)\rangle_{j}=-i \frac{\delta}{\delta j(x)} S_{\text {grav }}^{\text {on-shell }}=-i \lim _{z \rightarrow 0} z^{d-\Delta} \frac{\delta}{\delta \Phi(x, z)} S_{\text {grav }}^{\text {on-shell }}
$$

and thus

$$
\langle\mathcal{O}(x)\rangle_{j} \sim \Phi_{+}(x)
$$

One finds that $\Phi_{+}(x)$ is related to the expectation values of $\mathcal{O}$ in the presence of the source $j$ [372]. The exact relation depends on the normalization of the fields chosen [339]. The field $\Phi_{+}$thus acts as a classical field, and it is the boundary limit of the normalizable string solution which propagates in the bulk.

In the bottom-up phenomenological approach, the effective action in the bulk is usually modified for large values of $z$ to incorporate confinement and is usually truncated at the quadratic level.

\section{F.2 Two-point functions for arbitrary spin and Migdal procedure}

We start from the action (D.6). Performing a partial integration in the variable $z$ and using the equation of motion (D.8) one obtains, after a Fourier transform in $x$, the on-shell gravity action

$$
S_{\text {grav }}^{\text {on-shell }}\left[\hat{\Phi}^{\mathrm{cl}}\right]=\int d^{4} q\left(\frac{R}{z}\right)^{d-1} e^{\varphi(z)} \epsilon^{\nu_{1} \ldots \nu_{J}}(-q) \hat{\Phi}_{J}(-q, z) \epsilon_{\nu_{1} \ldots \nu_{J}}(q) \partial_{z} \hat{\Phi}_{J}(q, z)
$$

\footnotetext{
${ }^{1}$ For a description of the correlators of spinor and vector fields see for example Ref. [175].
} 
where $\epsilon_{\nu_{1} \ldots \nu_{J}}(q)$ is the polarization vector with

$$
q^{\nu_{1}} \epsilon_{\nu_{1} \ldots \nu_{J}}=0, \quad \eta^{\nu_{1} \nu_{2}} \epsilon_{\nu_{1} \nu_{2} \ldots \nu_{J}}=0
$$

and $\hat{\Phi}_{J}(q, z)$ is the solution of the equation of motion in the inertial frame (See (D.8)):

$$
\left[-q^{2}-\frac{z^{d-1}}{e^{\varphi}} \partial_{z}\left(\frac{e^{\varphi}}{z^{d-1}} \partial_{z}\right)+\frac{\left(\mu_{e f f}(z) R\right)^{2}+J}{z^{2}}\right] \hat{\Phi}(q, z)=0 .
$$

We go back to covariant tensor with $\Phi_{J}=\left(\frac{R}{z}\right)^{d-1} \hat{\Phi}$ (See (D.3)),

$$
\begin{aligned}
S_{\text {grav }}^{\text {on-shell }}\left[\Phi^{\mathrm{cl}}\right]=\int & d^{4} q\left(\frac{R}{z}\right)^{d-1-2 J} e^{\varphi(z)}\left(\epsilon^{\nu_{1} \ldots \nu_{J}}(-q) \Phi_{J}(-q, z)\right. \\
& \left.\times \epsilon_{\nu_{1} \ldots \nu_{J}}(q) \partial_{z} \Phi_{J}(q, z)+\frac{J}{z} \epsilon^{\nu_{1} \ldots \nu_{J}}(-q) \Phi_{J}(-q, z) \epsilon_{\nu_{1} \ldots \nu_{J}}(q) \Phi_{J}(q, z)\right) .
\end{aligned}
$$

In order to satisfy the condition $\Phi^{\mathrm{cl}}[q, z \rightarrow 0]=j(q)$ we put:

$$
\Phi_{J}^{\mathrm{cl}}(q, z) \epsilon_{\nu_{1} \ldots \nu_{J}}=j_{\nu_{1} \ldots \nu_{J}}(q) \lim _{\epsilon \rightarrow 0} \frac{\Phi_{J}(q, z)}{\Phi_{J}(q, \epsilon)} .
$$

We then obtain

$$
\begin{aligned}
S_{\text {grav }}^{\text {on-shell }}\left[\Phi^{\mathrm{cl}}\right]=\int d^{4} q\left(\frac{R}{z}\right)^{d-1-2 J} e^{\varphi(z)}\left(\frac{\partial_{z} \Phi(q, z)}{\Phi(q, \epsilon)}\right. & \left.+\frac{J}{z}\right) \\
& \times \sigma_{\nu_{1} \nu_{1}^{\prime} \ldots \nu_{J} \nu_{J}^{\prime}}(q) j^{\nu_{1} \ldots \nu_{J}}(-q) j^{\nu_{1}^{\prime} \ldots \nu_{J}^{\prime}}(q),
\end{aligned}
$$

where the spin tensor $\sigma_{\nu_{1} \nu_{1}^{\prime} \ldots \nu_{J} \nu_{J}^{\prime}}(q)$ reflects the conditions (F.13). The term $J / z$ in the action is independent of $q$ and therefore gives only rise to a contact term; it will, like all contact terms, be discarded in the following.

The propagator of the quantum field $\phi$ is obtained from $\log Z[j]$ by functional differentiation using the equality (F.7)

$$
\begin{aligned}
\left\langle\phi_{\nu_{1} \ldots \nu_{J}}(q) \phi_{\nu_{1}^{\prime} \ldots \nu_{J}^{\prime}}(-q)\right\rangle & =\frac{\delta}{\delta j^{\nu_{1} \ldots \nu_{J}(-q)}} \frac{\delta}{\delta j^{\nu_{1}^{\prime} \ldots \nu_{J}^{\prime}}(q)} \log Z[j] \\
& =2 i \sigma_{\nu_{1} \nu_{1}^{\prime} \ldots \nu_{J} \nu_{J}^{\prime}}(q)\left(\frac{R}{z}\right)^{3-2 J} e^{\varphi(z)} \frac{\partial_{z} \Phi(q, z)}{\Phi(q, \epsilon)} \\
& \equiv i \sigma_{\nu_{1} \nu_{1}^{\prime} \ldots \nu_{J} \nu_{J}^{\prime}}(q) \Sigma\left(q^{2}\right) .
\end{aligned}
$$


For the conserved vector current $(\mu=0 ; J=L=1)$ one starts with the AdS action

$$
S_{\mathrm{AdS}}=\frac{1}{2} \int d^{4} x d z\left(\frac{R}{z}\right)^{5} F^{M N} F_{M N} .
$$

In the hard wall model with Dirichlet boundary conditions at $z_{0}$ one obtains [99]

$$
\frac{1}{R} \Sigma\left(q^{2}\right)=2 q^{2} \lim _{z \rightarrow 0}\left(\log (q z)-\frac{\pi Y_{1}\left(q z_{0}\right)}{2 J_{1}\left(q z_{0}\right)}\right)
$$

For the soft wall model the solutions are

$$
\Phi(z)=z^{2+L-J} e^{-(\lambda+|\lambda|) z^{2} / 2} U\left(a, L+1,|\lambda| z^{2}\right),
$$

with $a=\frac{1}{4}\left(\frac{-q^{2}}{|\lambda|}+2 L+2-\frac{\lambda}{|\lambda|}(2-2 J)\right)$. The function $U(a, b, z)$ in (F.20), is the solution of Kummer's equation which vanishes for $z \rightarrow \infty$ [373]. One obtains for the propagator of the conserved current in the soft wall model [103, 104]

$$
\frac{1}{R} \Sigma\left(q^{2}\right)=-q^{2} \psi\left(-\frac{q^{2}}{4 \lambda}+1\right)-q^{2} \log \left(|\lambda| z^{2}\right)
$$

where $\psi(z)$ is the meromorphic Digamma function, $\psi(z)=\partial_{z} \log (\Gamma(z))$ which has poles at $z=0,-1,-2 \cdots$. The term which is infinite in the limit $z \rightarrow 0$ is a contact term, which will be discarded.

As has been shown in [99] the propagator of the conserved vector current obtained in the hard-wall model of $\mathrm{AdS}_{5}$, Eq. (F.19), is identical to the result obtained by Migdal $[100,101]$ in its hadronization scheme of perturbative QCD. In the $N_{C} \rightarrow \infty$ limit of QCD the hadronic cuts should vanish and only the hadron poles survive. Furthermore conformal invariance makes it possible to find explicit perturbative expressions up to order $\alpha_{s}^{4}$ which are diagonal in the rank of the hadron interpolating operators [374, 375]. Migdal has constructed propagators which asymptotically reproduce the perturbative result, but have only poles on the positive real axis of $q^{2}$. As a prescription to obtain this answer, he used the Padé approximation where the logarithm of the perturbative result is approximated by a quotient of power series. As stressed in [99] the hard-wall model of $\mathrm{AdS}_{5}$ is a convenient framework to achieve this "meromorphization". The logarithmic cuts occurring in the Bessel function $Y_{1}$ are cancelled by the logarithmic term in (F.19). It was considered as a deficiency that the Migdal regularization scheme did not lead to 
linear Regge trajectories and induced Migdal not to pursue this investigations further ${ }^{2}$.

Insisting on equally spaced poles on the real axis (Regge daughters) an obvious choice for a meromorphization is the digamma function $\psi(z)$, see (F.21). It has poles at $z=0,-1,-2 \cdots$ and behaves in the deep Euclidean region $z \gg 1$ like $\log z^{2}$. Therefore, one can also see the soft-wall model of $\mathrm{AdS}_{5}$ as a correspondence of the meromorphization program of perturbative QCD. Since the asymptotic expansion of the digamma function is

$$
\psi(z) \sim \log z-\frac{1}{2 z}-\sum_{n=1}^{\infty} \frac{B_{2 n}}{2 n z^{2 n}}=\log z-\frac{1}{2 z}-\frac{1}{12 z^{2}}+\ldots, \quad z \rightarrow \infty,|\arg z|<\pi
$$

the soft wall model is closer to the QCD sum rule method of Shifman, Vainshtein and Zakharov [102] than to the Migdal model [103, 104]. Also in the sum rule approach, corrections with inverse powers of $Q^{2}$ are added to the perturbative expression in order to improve the perturbative result in the not so deep Euclidean region. Note that there is also a power correction proportional to $1 / Q^{2}$ which does not correspond to a classical vacuum expectation value (See [376]). Finite-energy sum rules also lead under model assumptions to a linear rise in $M^{2}$ for the radial excitations: $M^{2} \sim n[377,378]$.

Next we discuss the fields with $L=0{ }^{3}$ which play an important role in the phenomenology of LF holographic QCD, where $\lambda>0$. For the vector current which interpolates the $\rho$ in light-front $\mathrm{AdS} / \mathrm{QCD}$, i.e. the field with quantum numbers $J=1, L=0$, $\left((\mu R)^{2}=-1\right)$ one obtains in the soft wall model (up to finite and diverging contact terms)

$$
\frac{1}{R} \Sigma\left(q^{2}\right)=\frac{1}{(z \log (z))^{2}}\left[\psi\left(-\frac{q^{2}}{4 \lambda}+\frac{1}{2}\right)+O\left(\frac{1}{\log z}\right)\right]+O(1) .
$$

The leading term in the expression for the generating functional $\log Z[j]$, proportional to the square bracket in (F.23) is a meromorphic function with the poles as predicted by the equation of motion. The gauge gravity prescription $[27,28]$ given by (F.7) is equivalent to

$$
\log Z[j]=\lim _{z \rightarrow 0}(z \log z)^{2} i S_{\mathrm{grav}}^{\mathrm{on}-\operatorname{shell}}\left[\Phi_{[z=\epsilon]}^{\mathrm{cl}} \rightarrow j\right]
$$

The factor $z^{2} \log ^{2} z$ cancels the infinity in (F.23) as $z \rightarrow \infty$ and the $O(1)$ contributions vanish. Note that in this case the polarization tensor is given by $g_{\mu \nu}-q_{\mu} q_{\nu} / q^{2}$.

\footnotetext{
${ }^{2}$ M. Shifman, private communication

${ }^{3}$ For a treatment in the soft wall model with negative dilaton profile see $[220,379]$.
} 
For a general tensor field with $L>0$ we obtain:

$$
\Sigma\left(q^{2}\right)=C_{L} R^{3-2 J} z^{2(J+L)-4} \lambda^{L} \frac{\Gamma\left(\frac{-q^{2}+2(J+L) \lambda}{4 \lambda}\right)}{\Gamma\left(\frac{-q^{2}+2(J-L) \lambda}{4 \lambda}\right)} \psi\left(\frac{-q^{2}+2(J+L) \lambda}{4 \lambda}\right),
$$

where $C_{L}$ is an $L$-dependent number. The ratio of the Gamma functions is a polynomial in $q^{2}$ of degree $L$. For $L>0$ the relation (F.7) becomes

$$
\log Z[j]=\lim _{z \rightarrow 0}(z)^{4-2(J+L)} i S_{\text {grav }}^{\text {on-shell }}\left[\Phi_{[z=\epsilon]}^{\mathrm{cl}} \rightarrow j\right]
$$

In the hard-wall model we obtain similar results as in the soft-wall model, but the meromorphic digamma function is replaced by a meromorphic combination of $J$ and $Y$ Bessel functions. Specifically for the case $L>0$ we obtain:

$$
\Sigma\left(q^{2}\right)=C_{L}^{\prime} R^{3-2 J} z^{2(J+L)-4} q^{2 L} \frac{\pi Y_{L}\left(q z_{0}\right)-2 \log \left(q z_{0}\right) J_{L}\left(q z_{0}\right)}{J_{L}\left(q z_{0}\right)} .
$$

To summarize: Both the soft- and hard-wall models in LF holographic QCD can be seen as a meromorphization of the perturbative QCD expression. The philosophy of the two procedures is, however, quite different. The Migdal procedure, corresponding to the hard-wall model, stays as close as possible to perturbative QCD. The Padé approximation is chosen in such a way as to optimize the agreement with the perturbative expression in the deep Euclidean region. In the soft-wall model, where the two-point function is proportional to the digamma function, there are power corrections to the (perturbative) logarithm, see (F.22). Therefore, the soft-wall model is closer in spirit to the QCD sum rule method [102] where also power corrections are added to the perturbative result $[103,104]$. The essential input is the equal spacing of the Regge daughters as in the Veneziano model [380]. The slope of the trajectory fixes the genuine nonperturbative quantity, the scale $\lambda$. Finally, let us remark that both the hard- and the soft-wall models share essential features of QCD in the $N_{C} \rightarrow \infty$ limit: The propagators are meromorphic functions and the higher $n$-point functions vanish. 


\section{Appendix G}

\section{Some Useful Formulæ}

\section{G.1 Solutions of the equations of motion in AdS space}

The equation of motion for arbitrary spin has the generic form given by (4.23)

$$
-\frac{z^{k}}{e^{\varphi(z)}} \partial_{z}\left(\frac{e^{\varphi(z)}}{z^{k}} \partial_{z} \Phi(z)\right)+\left(\frac{(m R)^{2}}{z^{2}}-M^{2}\right) \Phi(z)=0
$$

where $k=d-2 J-1$. This equation can be rewritten as

$$
\left(-z^{2} \partial_{z}^{2}+\left(k-z \varphi^{\prime}(z)\right) z \partial_{z}+(m R)^{2}-z^{2} M^{2}\right) \Phi(z)=0
$$

By rescaling the field according to $\Phi(z)=z^{k / 2} e^{-\varphi(z) / 2} \phi(z)$ this equation can be brought into a Schrödinger form

$$
\left(-\frac{d^{2}}{d z^{2}}+\frac{2 k+k^{2}+4(m R)^{2}}{4 z^{2}}+\frac{z^{2} \varphi^{2}+2 z^{2} \varphi^{\prime \prime}-2 k z \varphi^{\prime}}{4 z^{2}}\right) \phi(z)=M^{2} \phi(z),
$$

which shows the structure of the light-front Hamiltonian.

For the hard-wall model one has $\varphi(z)=0$ and can, for $M^{2}>0$ bring (G.2) into the form of a Bessel equation [373] by rescaling $\Phi(z)=z^{(k+1) / 2} f(y), \quad y=M z$ :

$$
y^{2} f^{\prime \prime}(y)+y f^{\prime}(y)+\left(y^{2}-\nu^{2}\right) f(y)=0
$$

with $\nu^{2}=(m R)^{2}+\frac{1}{4}(k+1)^{2}$. Solutions are the Bessel functions of the first and second 
kind, $J_{\nu}(y)$ and $Y_{\nu}(y)$. Only the Bessel functions of the first kind are regular at $z=0$

$$
J_{\nu}(y)=\frac{(y / 2)^{\nu}}{\Gamma(\nu+1)}+O\left(y^{2(\nu+1)}\right), \quad \nu \neq-1,-2, \cdots
$$

For normalization one can use the integral formula

$$
\int_{0}^{y_{0}} d y y J_{L}^{2}(y)=\frac{1}{2} y_{0}^{2}\left(\left(J_{L}^{2}\left(y_{0}\right)-J_{L-1}\left(y_{0}\right) J_{L+1}\left(y_{0}\right)\right) .\right.
$$

In the hard wall model $J_{\nu}\left(M z_{0}\right)=0$ and the zeroes of the Bessel functions $J_{\nu}(y)$ determine the hadron spectrum: Each Bessel function has an infinite set of zeroes. For small values of $\nu$ we can use the approximation [373]

$$
\beta_{\nu, r} \approx\left(k+\frac{\nu}{2}-\frac{1}{4}\right) \pi-\frac{4 \nu^{2}-1}{8 \pi\left(r+\frac{\nu}{2}-\frac{1}{4}\right)} .
$$

For the case $M^{2}=-Q^{2}<0$ we obtain by rescaling $\Phi(z)=z^{(k+1) / 2} f(y), y=Q z$ the modified Bessel equation

$$
y^{2} f^{\prime \prime}(y)+y f^{\prime}(y)-\left(y^{2}+\nu^{2}\right) f(y)=0,
$$

with $\nu^{2}=(m R)^{2}-\frac{1}{4}(k+1)^{2}$. Its two independent solutions are the modified Bessel functions $I_{\nu}(y)$ and $K_{\nu}(y)$ [373]. The function $I_{\nu}(y)$ increases asymptotically like $e^{y}$ for $y \rightarrow \infty, K_{\nu}(y)$ is singular at $y=0, K_{0}(y)$ diverges logarithmically at $y=0$.

In the case of the soft-wall model one has the dilaton profile $\varphi(z)=\lambda z^{2}$ and (G.2) can be brought into the following form by rescaling $\Phi(z)=z^{(k+1) / 2} e^{-\lambda z^{2} / 2} f(z)$ :

$$
\left(-\frac{d^{2}}{d z^{2}}-\frac{1}{z} \frac{d}{d z}+\frac{L^{2}}{z^{2}}+\lambda^{2} z^{2}\right) f(z)=\left(M^{2}+(k-1) \lambda\right) f(z)
$$

with $L^{2}=(m R)^{2}+\frac{(k+1)^{2}}{4}$.

The operator of the left hand side of (G.9) is the Hamiltonian of an harmonic oscillator in two dimensions with angular momentum $L$. The normalized eigenfunctions of the harmonic oscillator are

$$
f_{n L}(z)=\sqrt{\frac{2 n !}{(n+L) !}} z^{L} e^{-|\lambda| z^{2} / 2} L_{n}^{L}\left(|\lambda| z^{2}\right)
$$


and its eigenvalues are $E_{n L}=(2 n+L+1)|\lambda|$. The spectrum of eigenvalues for $M^{2}$ is thus given by

$$
M_{n L}^{2}=(4 n+2 L+2)|\lambda|-(k-1)|\lambda|
$$

If one rescales $\Phi(z)=z^{(k+1) / 2+L} g(y)$ with $y=|\lambda| z^{2}$ and $L^{2}=(m R)^{2}+\frac{(k+1)^{2}}{4}$ one obtains the equation

$$
y g^{\prime \prime}(y)+\left(b+\frac{\lambda}{|\lambda|} y\right) g^{\prime}(y)-a g(y)=0,
$$

with $a=-\frac{1}{4} \frac{\lambda}{|\lambda|}(2 L+k+1)-\frac{M^{2}}{4|\lambda|}, \quad b=L+1$.

For $\lambda<0$ this is Kummer's equation [373] with the solutions $M(a, b, y)$ and $U(a, b, y)$. $M(a, b, z)$ increases exponentially for $z \rightarrow+\infty$ and leads to a divergent solution; thus, only the hypergeometric function $U$ is of interest for us. For $a=-n$, the confluent hypergeometric function $U$ is regular at $z=0$ and is given by [373]

$$
U(-n, L+1, y)=\frac{n !}{(L+1)(L+2) \cdots(L+n)} L_{n}^{L}(y)
$$

The condition $a=-n$ yields the spectrum (G.11) for $\lambda<0$; one thus recovers the result obtained above. Equation (G.12) is valid for arbitrary values of $a$ and hence also for negative values of $M^{2}=-Q^{2}$.

For $\lambda>0$ on can transform (G.12) into Kummer's equation by additional rescaling by the factor $e^{-\lambda z^{2}}$, that is $\Phi(z)=z^{(k+1) / 2+L} e^{-\lambda z^{2}} g(y), y=|\lambda| z^{2}$. Then one obtains from (G.2) Kummer's equation (G.12), but with $a=\frac{1}{4}(2 L+3-k)-\frac{M^{2}}{4 \lambda}$.

For the electromagnetic current $(d=4)$ we have $k=1, L=1$ and the the solution of (G.12) is [373]

$$
g(y)=U\left(1+\frac{Q^{2}}{4|\lambda|}, 2, y\right)=\frac{1}{y} U\left(\frac{Q^{2}}{4|\lambda|}, 0, y\right)
$$

The solution $\Phi$, normalized to $\Phi(0)=1$ can be written as

$$
\Phi(z)=\Gamma\left(1+\frac{Q^{2}}{4|\lambda|}\right) e^{-(\lambda+|\lambda|) z^{2} / 2} U\left(\frac{Q^{2}}{4|\lambda|}, 0,|\lambda| z^{2}\right) .
$$

The solutions of the differential equations relevant for the soft- and hard-wall models are summarized in Table G.1. 


\section{G.1.1 A useful integral}

The integral relation between the Bessel function of the first kind $J_{\alpha}(x)$ and the modified Bessel function $K_{\alpha}(x)$ is usually given by the Hankel-Nicholson integral [373]

$$
\int_{0}^{\infty} \frac{t^{\nu+1} J_{\nu}(z t)}{\left(t^{2}+a^{2}\right)^{\mu+1}} d t=\frac{z^{\mu} a^{\nu-\mu}}{2^{\mu} \Gamma(\mu+1)} K_{\nu-\mu}(a z)
$$

Changing the variable $t$ according to $x=\frac{a^{2}}{t^{2}+a^{2}}$ we can recast the integral (G.16) as

$$
\int_{0}^{1} d x x^{\mu-1}\left(\frac{1-x}{x}\right)^{\nu / 2} J_{\nu}\left(a z \sqrt{\frac{1-x}{x}}\right)=\frac{(a z)^{\mu}}{2^{\mu-1} \Gamma(\mu+1)} K_{\nu-\mu}(a z) .
$$




\begin{tabular}{l|l}
\hline \hline \multicolumn{1}{c}{$\varphi \equiv 0$} \\
\hline$M^{2}>0$ & $\Phi(z)=z^{d / 2-J}\left(A J_{L}(M z)+B Y_{L}(M z)\right)$ \\
& $\phi(z)=\frac{1}{N} z^{1 / 2} J_{L}(M z)$ \\
& $N^{2}=z_{0}^{2}\left(J_{L}\left(M z_{0}\right)^{2}-J_{L-1}\left(M z_{0}\right) J_{L+1}\left(M z_{0}\right)\right)$ \\
& $M_{n L}=\beta_{L k} / z_{0}$
\end{tabular}

Table G.1: General form of the solutions of the AdS wave equations for integer spin and their spectra. The AdS field $\Phi$ denotes the solution of Eq. (4.23). The solution $\phi$ is rescaled as $\phi=z^{(2 J+1-d) / 2} e^{\varphi(z) / 2} \Phi$ in order to satisfy an equation of the Schrödinger type (G.3). The normalization factor $N$ is determined from $\int d z \phi^{2}(z)=1$ and is regular at $z=0$. 


\section{Appendix $\mathbf{H}$}

\section{Integrability and Light-Front Effective Hamiltonians}

Integrability of a physical system is related to its symmetries. In holographic QCD the conformal symmetry often means integrability: the solution to the differential equations describing the system can be expressed is terms of analytical functions. In this appendix we shall follow the remarkable integrability methods introduced by L. Infeld in a 1941 paper [381, 382]. The key observation in Infeld's paper is the realization that integrability follows immediately if the equation describing a physical model can be factorized in terms of linear operators. These operators, the ladder operators, generate all the eigenfunctions once the basic eigenfunction is know. In the following we will describe how to construct effective light-front Hamiltonians corresponding to the hard and soft-wall models discussed in this report from the algebra of bosonic or fermionic linear operators $[138,257]$. In particular, we describe here a different approach to the soft-wall model which results from a minimal extension of the conformal algebraic structures. This method is particularly useful in the fermionic sector where the corresponding linear wave equations become exactly solvable [138, 257]. 


\section{H.1 Light-front effective bosonic Hamiltonians}

\section{H.1.1 Light-front hard-wall model}

To illustrate the algebraic construction procedure consider first, as a simple example, the light-front Hamiltonian form (5.2) in the conformal limit:

$$
H_{L F}^{\nu}=-\frac{d^{2}}{d \zeta^{2}}-\frac{1-4 \nu^{2}}{4 \zeta^{2}}
$$

with hadronic mass eigenvalues and eigenstates determined by the eigenvalue equation

$$
H_{L F}^{\nu} \phi_{\nu}(\zeta)=M_{\nu}^{2} \phi_{\nu}(\zeta)
$$

If $\nu>0$ the Hamiltonian (H.1) can be expressed as a bilinear form

$$
H_{L F}^{\nu}=b_{\nu} b_{\nu}^{\dagger}
$$

where

$$
b=\frac{d}{d \zeta}+\frac{\nu+\frac{1}{2}}{\zeta}
$$

and its adjoint

$$
b^{\dagger}=-\frac{d}{d \zeta}+\frac{\nu+\frac{1}{2}}{\zeta}
$$

with $\left(\frac{d}{d \zeta}\right)^{\dagger}=-\frac{d}{d \zeta}$

Since the Hamiltonian is a bilinear form, its eigenvalues are positive definite

$$
\left\langle\phi\left|H_{L F}^{\nu}\right| \phi\right\rangle=\int d \zeta\left|b_{\nu}^{\dagger} \phi(z)\right|^{2} \geq 0
$$

Consequently $M^{2} \geq 0$ if $\nu^{2} \geq 0$. The critical value $\nu=0$ corresponds to the lowest possible stable solution. If $\nu^{2}<0$ the system is not bounded from below.

From the eigenvalue equation (H.2) we obtain the wave equation

$$
\left(-\frac{d^{2}}{d x^{2}}-\frac{1-4 \nu^{2}}{4 x^{2}}\right) \phi_{\nu}(x)=\phi_{\nu}(x),
$$


where $x=\zeta M$. In terms of the operators $b_{\nu}$ and $b_{\nu}^{\dagger}$ (H.6) is equivalent to

$$
b_{\nu} b_{\nu}^{\dagger}|\nu\rangle=|\nu\rangle .
$$

Multiplying both sides on the left by $b_{\nu}^{\dagger}$,

$$
b_{\nu}^{\dagger} b_{\nu}\left\{b_{\nu}^{\dagger}|\nu\rangle\right\}=\left\{b_{\nu}^{\dagger}|\nu\rangle\right\} .
$$

It is simple to verify that

$$
b_{\nu}^{\dagger} b_{\nu}=b_{\nu+1} b_{\nu+1}^{\dagger},
$$

and thus

$$
b_{\nu+1} b_{\nu+1}^{\dagger}\left\{b_{\nu}^{\dagger}|\nu\rangle\right\}=\left\{b_{\nu}^{\dagger}|\nu\rangle\right\} .
$$

Consequently

$$
b_{\nu}^{\dagger}|\nu\rangle=c_{\nu}|\nu+1\rangle,
$$

or

$$
\left(-\frac{d}{d x}+\frac{\nu+\frac{1}{2}}{x}\right) \phi_{\nu}(x)=c_{\nu} \phi_{\nu+1}(x),
$$

with $c_{\nu}$ a constant. Thus $b_{\nu}^{\dagger}$ is the raising operator. Likewise, one can show that $b_{\nu}$ is the lowering operator,

$$
b_{\nu}|\nu+1\rangle=c_{\nu}|\nu\rangle,
$$

or

$$
\left(\frac{d}{d x}+\frac{\nu+\frac{1}{2}}{x}\right) \phi_{\nu+1}(x)=c_{\nu} \phi_{\nu}(x),
$$

with $c_{\nu}$ a constant.

Writing

$$
\phi_{\nu}(x)=C \sqrt{x} F_{\nu}(x),
$$

and substituting in (H.12) we get

$$
\frac{\nu}{x} F_{\nu}(x)-F_{\nu}^{\prime}(x) \sim F_{\nu+1}(x),
$$

a relation which defines a Bessel function $Z_{\nu+1}(x)$ of rank $\nu+1$ in terms of a Bessel of rank $\nu, Z_{\nu}(z),[373]$

$$
\frac{\nu}{x} Z_{\nu}(x)-Z_{\nu}^{\prime}(x)=Z_{\nu+1}(x) .
$$


Thus the normalizable solution to the eigenvalue equation (H.2) is

$$
\phi_{\nu}(\zeta)=C_{\nu} \sqrt{z} J_{\nu}(\zeta M)
$$

with $C_{\nu}$ a constant. The eigenvalues are obtained from the boundary conditions and are given in terms of the roots of the Bessel functions.

\section{H.1.2 Light-front soft-wall model}

We can introduce a scale by modifying the operators H.4 and H.5 while keeping an integrable system. Let us consider the extended operator

$$
b_{\nu}=\frac{d}{d \zeta}+\frac{\nu+\frac{1}{2}}{\zeta}+\lambda \zeta
$$

and its adjoint

$$
b_{\nu}^{\dagger}=-\frac{d}{d \zeta}+\frac{\nu+\frac{1}{2}}{\zeta}+\lambda \zeta
$$

Since we have introduced a scale $\lambda$ in the problem, the effective Hamiltonian has the general form

$$
H_{L F}^{\nu}=b_{\nu} b_{\nu}^{\dagger}+C(\lambda)
$$

where the constant term $C(\lambda)$ depends on the spin representations. Since the Hamiltonian is a bilinear form, modulo a constant, its eigenvalues are positive definite $M^{2} \geq 0$ provided that $\nu^{2} \geq 0$ and $C(\lambda) \geq-4 \lambda$.

Let us consider the case where the ground state is massless (the pion). In this case $C(\lambda)=-4 \lambda$ and the LF effective Hamiltonian is given by

$$
H_{L F}^{\nu}(\zeta)=-\frac{d^{2}}{d \zeta^{2}}-\frac{1-4 \nu^{2}}{4 \zeta^{2}}+\lambda^{2} \zeta^{2}+2 \lambda(\nu-1)
$$

which is identical to the LF Hamiltonian from (5.2) with effective potential (5.5),

$$
U(\zeta)=\lambda^{2} \zeta^{2}+2 \lambda(\nu-1)
$$

for $\nu=J=L$.

Following the analysis of Sec. H.1.1 it is simple to show that the operator $b_{\nu}^{\dagger}$ acts as the creation operator,

$$
b_{\nu}^{\dagger}|\nu\rangle=c_{\nu}|\nu+1\rangle,
$$


or

$$
\left(-\frac{d}{d \zeta}+\frac{\nu+\frac{1}{2}}{\zeta}+\lambda \zeta\right) \phi_{\nu}(\zeta)=c_{\nu} \phi_{\nu+1}(\zeta)
$$

with $c_{\nu}$ a constant.

We also consider the operator

$$
a_{\nu}=-\frac{d}{d \zeta}+\frac{\nu+\frac{1}{2}}{\zeta}-\lambda \zeta
$$

and its adjoint

$$
a_{\nu}^{\dagger}=\frac{d}{d \zeta}+\frac{\nu+\frac{1}{2}}{\zeta}-\lambda \zeta .
$$

It is also simple to verify that the operator $a_{\nu}$ lowers the radial quantum number $n$ by one unit and raises $\nu$ by one unit

$$
a_{\nu}|n, \nu\rangle \sim|n-1, \nu+1\rangle
$$

Notice that the state $|n-1, \nu+1\rangle$ obtained by application of the operator $a_{\nu}$ is degenerate with the state $|n, \nu\rangle$. For a given $\nu$ the lowest possible state corresponds to $n=0$. Consequently the state $|n=0, \nu\rangle$ is annihilated by the action of the operator $a_{\nu}$

$$
a_{\nu}|n=0, \nu\rangle=0
$$

or equivalently

$$
\left(\frac{d}{d \zeta}-\frac{\nu+\frac{1}{2}}{\zeta}+\lambda \zeta\right) \phi_{\nu}^{n=0}(\zeta)=0
$$

with solution

$$
\phi_{\nu}^{n=0}(\zeta)=C_{\nu} \zeta^{1 / 2+\nu} e^{-\lambda \zeta^{2} / 2}
$$

where $C_{\nu}$ is a constant. Writing

$$
\phi_{\nu}(\zeta)=C_{\nu} \zeta^{1 / 2+\nu} e^{-\lambda \zeta^{2} / 2} G_{\nu}(\zeta)
$$

and substituting in (H.25) we get

$$
2 x G_{\nu}(x)-G^{\prime}(x) \sim x G_{\nu+1}(x),
$$

with $x=\sqrt{\lambda} \zeta$, a relation which defines the confluent hypergeometric function $U(n, \nu+$ 
$1, x)$ in terms of $U(n, \nu, x)[373]$

$$
U(n, \nu, x)-U^{\prime}(n, \nu, x)=U(n, \nu+1, x),
$$

or equivalently

$$
2 x U\left(n, \nu, x^{2}\right)-\frac{d U\left(n, \nu, x^{2}\right)}{d x}=2 x U\left(n, \nu+1, x^{2}\right) .
$$

Thus the normalizable solution of the eigenvalue equation $b_{\nu} b_{\nu}^{\dagger} \phi=M^{2} \phi$ is

$$
\phi_{n, \nu}(\zeta)=C_{\nu} \zeta^{1 / 2+\nu} e^{-\lambda \zeta^{2} / 2} L_{n}^{\nu}\left(\lambda \zeta^{2}\right)
$$

with $C_{\nu}$ a constant. The solution also follows from the iterative application of the ladder operators, the Rodriguez formula for the Laguerre polynomials (See [383]). We find

$$
\phi(\zeta)_{n, \nu} \sim \zeta^{1 / 2-\nu} e^{\lambda \zeta^{2} / 2}\left(\frac{1}{\zeta} \frac{d}{d \zeta}\right)^{n} \zeta^{2(n+\nu)} e^{-\lambda \zeta^{2}}
$$

with eigenvalues

$$
M^{2}=4 \lambda(n+\nu+1)
$$

\section{H.2 Light-front effective fermionic Hamiltonians}

In this section we extend the algebraic procedure described in Sec. H.1 to construct light-front effective Hamiltonians for LF baryonic modes. We will describe first the conformal case where we have an exact prescription from the mapping of AdS wave equations (See Secs. 4.3 and 5.2). Then, as for for the case of LF bosonic modes described above, we extend the conformal limiting case to include a scale while maintaining integrability of the Hamiltonian eigenvalue equations [138, 257]. This procedure turns out to be particularly useful since in AdS the confining dilaton background is absorbed by a rescaling of the Dirac field (Sec. 4.3), and thus we have little guidance in this case from the gravity side. However, as we shall show below, a consistent solution can be found by imposing the correct transformation properties for half-integer spin. 


\section{H.2.1 Light-front hard-wall model}

We consider an effective light-front Dirac-type equation to describe a baryonic state in holographic QCD. In the conformal limit

$$
\left(D_{L F}-M\right) \psi(\zeta)
$$

where the Dirac operator $D_{L F}$ is the hermitian operator

$$
D_{L F}=-i \alpha\left(-\frac{d}{d \zeta}+\frac{\nu+\frac{1}{2}}{\zeta} \gamma\right)
$$

and $\alpha$ and $\gamma$ are matrices to be determined latter.

It is useful to define the matrix-valued (non-Hermitian) operator

$$
\mathbf{b}=\frac{d}{d \zeta}+\frac{\nu+\frac{1}{2}}{\zeta} \gamma
$$

and its adjoint

$$
\mathbf{b}^{\dagger}=-\frac{d}{d \zeta}+\frac{\nu+\frac{1}{2}}{\zeta} \gamma
$$

Premultiplying the linear Dirac wave equation (H.60) by the operator $D_{L F}+M$ we should recover the LF Hamiltonian eigenvalue equation:

$$
H_{L F} \psi=D_{L F}^{2} \psi=M^{2} \psi
$$

which imply that

$$
\begin{array}{r}
\alpha^{\dagger}=\alpha, \quad \alpha^{2}=1 \\
\gamma^{\dagger}=\gamma, \quad \gamma^{2}=1 \\
\{\alpha, \gamma\}=0
\end{array}
$$

Consequently the matrices $\alpha$ and $\gamma$ are four-dimensional Dirac matrices.

The effective light-front Hamiltonian $H_{L F}=D_{L F}^{2}=\mathbf{b}_{\nu} \mathbf{b}_{\nu}^{\dagger}$ is thus given by

$$
H_{L F}^{\nu}=-\frac{d^{2}}{d \zeta^{2}}+\frac{\left(\nu+\frac{1}{2}\right)^{2}}{\zeta^{2}}-\frac{\nu+\frac{1}{2}}{\zeta^{2}} \gamma
$$

The positivity of the product of operators imply that $\left\langle\psi\left|H_{L F}^{\nu}\right| \psi\right\rangle \geq 0$, and thus $M^{2} \geq 0$ 
if $\nu^{2} \geq 0$, identical to the stability bound for the scalar case.

To satisfy the wave equation (H.39) for each component $\psi_{\alpha}$ we require that the matrix $\gamma$ satisfies the equation

$$
\gamma u_{ \pm}= \pm u_{ \pm}
$$

where $u_{ \pm}$are four-component chiral spinors. Consequently the matrix $\gamma$ is the four dimensional chirality operator $\gamma_{5}$. The LF equation (H.47) thus leads to the eigenvalue equation

$$
H_{L F} \psi_{ \pm}=M^{2} \psi_{ \pm}
$$

where

$$
\left(-\frac{d^{2}}{d \zeta^{2}}-\frac{1-4 \nu^{2}}{4 \zeta^{2}}\right) \psi_{+}(\zeta)=M^{2} \psi_{+}(\zeta)
$$

and

$$
\left(-\frac{d^{2}}{d \zeta^{2}}-\frac{1-4(\nu+1)^{2}}{4 \zeta^{2}}\right) \psi_{-}(\zeta)=M^{2} \psi_{-}(\zeta)
$$

These are two uncoupled equations for the upper and lower spinor components, $\psi_{+}$and $\psi_{-}$, with the solution

$$
\psi_{+}(\zeta) \sim \sqrt{\zeta} J_{\nu}(\zeta M), \quad \psi_{-}(\zeta) \sim \sqrt{\zeta} J_{\nu+1}(\zeta M)
$$

The plus and minus components are not independent since they must also obey the first order Dirac equation (H.39). In the Weyl representation where the chirality operator $\gamma$ is diagonal $(i \alpha=\gamma \beta)$ we have

$$
i \alpha=\left(\begin{array}{cc}
0 & I \\
-I & 0
\end{array}\right), \quad \beta=\left(\begin{array}{cc}
0 & I \\
I & 0
\end{array}\right), \quad \gamma=\left(\begin{array}{cc}
I & 0 \\
0 & -I
\end{array}\right)
$$

where $I$ a two-dimensional unit matrix. The linear equation (H.39) is equivalent to the system of coupled equations

$$
\begin{aligned}
-\frac{d}{d \zeta} \psi_{-}-\frac{\nu+\frac{1}{2}}{\zeta} \psi_{-} & =M \psi_{+} \\
\frac{d}{d \zeta} \psi_{+}-\frac{\nu+\frac{1}{2}}{\zeta} \psi_{+} & =M \psi_{-}
\end{aligned}
$$

a result which is identical with the results wich follow from the Dirac AdS wave equation in the conformal limit $[138,257]$ (See Sec. 5.2). Solving the coupled equations (H.54 - 
H.55) and making use of the relation between Bessel functions

$$
J_{\nu+1}(x)=\frac{\nu}{x} J_{\nu}(x)-J_{\nu}^{\prime}(x)
$$

we obtain the solution

$$
\psi(\zeta)=C \sqrt{\zeta}\left[J_{\nu}(\zeta M) u_{+}+J_{\nu+1}(\zeta M) u_{-}\right]
$$

with normalization

$$
\int d \zeta \psi_{+}^{2}(\zeta)=\int d \zeta \psi_{-}^{2}(\zeta)
$$

identical for the plus and minus components.

\section{H.2.2 Light-front soft-wall model}

We write the Dirac equation

$$
\left(D_{L F}-M\right) \psi(\zeta)=0
$$

and construct an extended LF Dirac operator $D_{L F}$ following the same procedure used for the bosonic case in Sec. H.1.2, where we introduced a scale by making the substitution $\frac{\nu+1 / 2}{\zeta} \rightarrow \frac{\nu+1 / 2}{\zeta}+\lambda \zeta$. Thus the extended Dirac operator

$$
D_{L F}=-i \alpha\left(-\frac{d}{d \zeta}+\frac{\nu+\frac{1}{2}}{\zeta} \gamma+\lambda \zeta \gamma\right)
$$

and the extended matrix-valued non-Hermitian operators

$$
\begin{aligned}
\mathbf{b} & =\frac{d}{d \zeta}+\frac{\nu+\frac{1}{2}}{\zeta} \gamma+\lambda \zeta \gamma, \\
\mathbf{b}^{\dagger} & =-\frac{d}{d \zeta}+\frac{\nu+\frac{1}{2}}{\zeta} \gamma+\lambda \zeta \gamma .
\end{aligned}
$$

The effective light-front Hamiltonian $H_{L F}=D_{L F}^{2}=\mathbf{b b}^{\dagger}$ is given by

$$
H_{L F}=-\frac{d^{2}}{d \zeta^{2}}+\frac{\left(\nu+\frac{1}{2}\right)^{2}}{\zeta^{2}}-\frac{\nu+\frac{1}{2}}{\zeta^{2}} \gamma+\lambda^{2} \zeta^{2}+\lambda(2 \nu+1)+\lambda \gamma,
$$

with $\gamma$ the chirality matrix $\gamma u_{ \pm}= \pm u$. 
The eigenvalue equation $H_{L F} \psi_{ \pm}=M^{2} \psi_{ \pm}$leads to the uncoupled light-front wave equations

$$
\begin{aligned}
& \left(-\frac{d^{2}}{d \zeta^{2}}-\frac{1-4 \nu^{2}}{4 \zeta^{2}}+\lambda^{2} \zeta^{2}+2(\nu+1) \lambda\right) \psi_{+}(\zeta)=M^{2} \psi_{+}(\zeta), \\
& \left(-\frac{d^{2}}{d \zeta^{2}}-\frac{1-4(\nu+1)^{2}}{4 \zeta^{2}}+\lambda^{2} \zeta^{2}+2 \nu \lambda\right) \psi_{-}(\zeta)=M^{2} \psi_{-}(\zeta),
\end{aligned}
$$

with solutions

$$
\begin{aligned}
& \psi_{+}(\zeta) \sim z^{\frac{1}{2}+\nu} e^{-\lambda \zeta^{2} / 2} L_{n}^{\nu}\left(\lambda \zeta^{2}\right), \\
& \psi_{-}(\zeta) \sim z^{\frac{3}{2}+\nu} e^{-\lambda \zeta^{2} / 2} L_{n}^{\nu+1}\left(\lambda \zeta^{2}\right),
\end{aligned}
$$

and eigenvalues

$$
M^{2}=4 \lambda(n+\nu+1),
$$

identical for both plus and minus eigenfunctions.

Using the $2 \times 2$ representation of the Dirac matrices given in the previous section we find the system of coupled linear equations

$$
\begin{aligned}
-\frac{d}{d \zeta} \psi_{-}-\frac{\nu+\frac{1}{2}}{\zeta} \psi_{-}-\lambda \zeta \psi_{-} & =M \psi_{+} \\
\frac{d}{d \zeta} \psi_{+}-\frac{\nu+\frac{1}{2}}{\zeta} \psi_{+}-\lambda \zeta \psi_{+} & =M \psi_{-} .
\end{aligned}
$$

This result is identical with the results from the AdS wave equation in presence of a potential $V(z)=\lambda z[138,257]$ (See Sec. 5.2).

Solving the coupled equations (H.69 - H.70) making use of the relation between associated Laguerre functions

$$
L_{n-1}^{\nu+1}(x)+L_{n}^{\nu}(x)=L_{n}^{\nu+1}(x),
$$

we find

$$
\psi(\zeta)=C z^{\frac{1}{2}+\nu} e^{-\lambda \zeta^{2} / 2}\left[L_{n}^{\nu}\left(\lambda \zeta^{2}\right) u_{+}+\frac{\sqrt{\lambda} \zeta}{\sqrt{n+\nu+1}} L_{n}^{\nu+1}\left(\lambda \zeta^{2}\right) u_{-}\right],
$$

with normalization

$$
\int d \zeta \psi_{+}^{2}(\zeta)=\int d \zeta \psi_{-}^{2}(\zeta)
$$


It is important to notice that, in contrast to the bosonic case, one cannot add a constant term to the light-front effective Hamiltonian with dependence on the spin representations for baryons. This constraint follows from the fact that the plus and minus components are not independent and obey the first order linear equation H.59. This additional requirement has the notable consequence that, in contrast to bosons, there is no spin-orbit coupling in the light-front holographic model for baryons as discussed in Chapters 4 and 5 . 


\section{Appendix I}

\section{Equations of Motion for p-Form Fields in AdS}

In this appendix we describe the properties of massive $p$-form fields propagating in AdS space. As we discussed in Chapter 4, obtaining the general form of the equations of motion for higher-spin fields in AdS space may become quite complex. It is thus useful to study the simplified structure of the differential equations of $p$-form fields in AdS, which for $p=0$ and $p=1$ represent spin 0 and spin 1 respectively (Sec. 4.1). The compact formalism of differential forms is particularly convenient to describe the solutions of higher $p$-form actions in $\mathrm{AdS}_{d+1}$ space. In this notation a $p$-form field $\mathbf{A}$ is

$$
\mathbf{A}=\mathcal{A}_{M_{1} M_{2} \cdots M_{p}} d x^{M_{1}} \wedge \cdots \wedge d x^{M_{p}},
$$

in the dual basis $d x^{M}$. The tensor field $\mathcal{A}_{M_{1} M_{2} \cdots M_{p}}$ is a totally antisymmetric tensor of rank $p$, and the sum is over $M_{1}<\cdots<M_{p}$. $\operatorname{AdS}_{d+1}$ coordinates are the Minkowski coordinates $x^{\mu}$ and $z$ which we label $x^{M}$, with $M, N=0, \cdots, d$.

The field strength $\mathbf{F}$ of the $p$ form $\mathbf{A}$ is the $p+1$ form given by the exterior derivative

$$
\mathbf{F}=d \mathbf{A}=\partial_{M} \mathcal{A}_{M_{1} M_{2} \cdots M_{p}} d x^{M_{1}} \wedge \cdots \wedge d x^{M_{p}} \wedge d x^{M},
$$

with sum over $M$ and $M_{1}<\cdots<M_{p}$. The invariant Lagrangian density must be a $d+1$ form. This leads to the action in geometrical units

$$
S=\frac{1}{2} \int_{A d S_{d+1}}\left(\mathbf{F} \wedge^{*} \mathbf{F}-\mu^{2} \mathbf{A} \wedge^{*} \mathbf{A}\right),
$$


where $\mu$ is the AdS mass.

The wedge product $\wedge$ of a $p$-form $\mathbf{A}$ and a $q$-form $\mathbf{B}$ is the $p+q$ form $\mathbf{A} \wedge \mathbf{B}$. In tensor notation:

$$
\mathbf{A} \wedge \mathbf{B}=\frac{(p+q) !}{p ! q !} A_{\left[M_{1} \ldots M_{p}\right.} B_{\left.M_{p+1} \ldots M_{p+q}\right]} d x^{M_{1}} \wedge \cdots \wedge^{d} x^{M_{p+q}}
$$

The hodge star operator establishes a correspondence between $p$-forms to $d+1-p$ forms on $\operatorname{Ad} S_{d+1}$. In tensor notation the Hodge dual of $\mathbf{A}$ is obtained by contracting the indices of $\mathbf{A}$ with the $d+1$-dimensional completely antisymmetric Levi-Civita tensor

$$
\left({ }^{*} \mathbf{A}\right)_{M_{1} M_{2} \cdots M_{d+1-p}}=\frac{1}{p !}(\mathbf{A})^{N_{1} N_{2} \cdots N_{p}} \eta_{N_{1} N_{2} \cdots N_{p}, M_{1} \cdots M_{d+1-p}},
$$

where the Levi-Civita tensor is

$$
\eta_{M_{1} \cdots M_{p}}=\sqrt{g} \epsilon_{M_{1} \cdots M_{p}}
$$

with $g \equiv\left|\operatorname{det} g_{M N}\right|$ and $\epsilon_{M_{1} \cdots M_{p}}$ the totally antisymmetric tensor density with entries \pm 1 .

The classical equations of motion for $\mathbf{A}$ follow from the variation of the action (I.3). Making use of the Stokes theorem we obtain:

$$
\int_{A d S_{d+1}} d\left(\mathbf{A} \wedge^{*} d \mathbf{A}\right)=0
$$

from which follows

$$
\int_{A d S_{d+1}}\left(d \mathbf{A} \wedge^{*} d \mathbf{A}\right)=(-1)^{p} \int_{A d S_{d+1}}\left(\mathbf{A} \wedge d^{*} d \mathbf{A}\right),
$$

yielding

$$
(-)^{p} d^{*} d \mathbf{A}+\mu^{2 *} \mathbf{A}=0
$$

Since $d d \mathbf{A}=\mathbf{0}$, this equation implies for $\mu \neq 0$

$$
d^{*} \mathbf{A}=0 \text {. }
$$

In tensor notation the equations of motion for $\mathbf{A}$ are expressed as the set of $p+1$ 
coupled differential equations [384]

$$
\begin{gathered}
{\left[z^{2} \partial_{z}^{2}-(d+1-2 p) z \partial_{z}-z^{2} \partial_{\rho} \partial^{\rho}-(\mu R)^{2}+d+1-2 p\right] \mathcal{A}_{z \alpha_{2} \cdots \alpha_{p}}=0} \\
{\left[z^{2} \partial_{z}^{2}-(d+1-2 p) z \partial_{z}-z^{2} \partial_{\rho} \partial^{\rho}-(\mu R)^{2}+d+1-2 p\right] \mathcal{A}_{\alpha_{1} z \cdots \alpha_{p}}=0} \\
\cdots \\
{\left[z^{2} \partial_{z}^{2}-(d-1-2 p) z \partial_{z}-z^{2} \partial_{\rho} \partial^{\rho}-(\mu R)^{2}\right] \mathcal{A}_{\alpha_{1} \alpha_{2} \cdots \alpha_{p}}=2 z\left(\partial_{\mu_{1}} \mathcal{A}_{z \alpha_{2} \cdots \alpha_{p}}\right.} \\
\left.+\partial_{\mu_{2}} \mathcal{A}_{\alpha_{1} z \cdots \alpha_{p}}+\cdots\right)
\end{gathered}
$$

where $\alpha, \rho=0,1,2, \cdots, d-1$ and the notation $z \alpha_{2} \cdots \alpha_{p}$ means $M_{1}=z, M_{2}=\alpha_{2}, \cdots$, $M_{p}=\alpha_{p}$, etc.

We introduce fields with Lorentz tangent indices $A, B=0, \cdots, d$,

$$
\hat{\mathcal{A}}_{A_{1} A_{2} \cdots A_{p}}=e_{A_{1}}^{M_{1}} e_{A_{2}}^{M_{2}} \cdots e_{A_{p}}^{M_{p}} \mathcal{A}_{M_{1} M_{2} \cdots M_{p}}=\left(\frac{z}{R}\right)^{p} \mathcal{A}_{A_{1} A_{2} \cdots A_{p}},
$$

where $e_{A}^{M}$ is the vielbein (See Sect. A.1.3). In terms of $\hat{\mathcal{A}}$ we obtain from (I.11-I.13) the set of $p+1$ differential equations [384]

$$
\begin{aligned}
{\left[z^{2} \partial_{z}^{2}-(d-1) z \partial_{z}-z^{2} \partial_{\rho} \partial^{\rho}-(\mu R)^{2}+p(d-p)\right]\left(\frac{\hat{\mathcal{A}}_{z \alpha_{2} \cdots \alpha_{p}}}{z}\right) } & =0 \\
{\left[z^{2} \partial_{z}^{2}-(d-1) z \partial_{z}-z^{2} \partial_{\rho} \partial^{\rho}-(\mu R)^{2}+p(d-p)\right]\left(\frac{\hat{\mathcal{A}}_{\alpha_{1} z \cdots \alpha_{p}}}{z}\right) } & =0 \\
\cdots & \\
{\left[z^{2} \partial_{z}^{2}-(d-1) z \partial_{z}-z^{2} \partial_{\rho} \partial^{\rho}-(\mu R)^{2}+p(d-p)\right] \hat{\mathcal{A}}_{\alpha_{1} \alpha_{2} \cdots \alpha_{p}} } & =2 z\left(\partial_{\mu_{1}} \hat{\mathcal{A}}_{z \alpha_{2} \cdots \alpha_{p}}\right. \\
\left.+\partial_{\mu_{2}} \hat{\mathcal{A}}_{\alpha_{1} z \cdots \alpha_{p}}+\cdots\right) . &
\end{aligned}
$$

Consider the plane-wave solution $\mathcal{A}_{P}\left(x^{\mu}, z\right)_{\alpha_{1} \cdots \alpha_{p}}=e^{i P \cdot x} \mathcal{A}(z)_{\alpha_{1} \cdots \alpha_{p}}$, with 4-momentum $P_{\mu}$, invariant hadronic mass $P_{\mu} P^{\mu}=M^{2}$ and spin indices $\alpha$ along the space-time coordinates, that is $\mathcal{A}_{z \alpha_{2} \cdots \alpha_{p}}=\mathcal{A}_{\alpha_{1} z \cdots \alpha_{p}}=\cdots=0$. In this case the system of coupled differential equations (I.11-I.13) reduce to the homogeneous wave equation

$$
\left[z^{2} \partial_{z}^{2}-(d-1-2 p) z \partial_{z}+z^{2} M^{2}-(\mu R)^{2}\right] \mathcal{A}_{\alpha_{1} \alpha_{2} \cdots \alpha_{p}}=0
$$

In tangent space the coupled differential equations (I.15-I.17) for all polarization indices along the Poincaré coordinates reduces to

$$
\left[z^{2} \partial_{z}^{2}-(d-1) z \partial_{z}+z^{2} M^{2}-(\mu R)^{2}+p(d-p)\right] \hat{\mathcal{A}}_{\alpha_{1} \alpha_{2} \cdots \alpha_{p}}=0
$$


Its solution is

$$
\tilde{\mathcal{A}}(z)_{\alpha_{1} \alpha_{2} \cdots \alpha_{p}}=C z^{\frac{d}{2}} J_{\Delta-\frac{d}{2}}(z M) \epsilon_{\alpha_{1} \alpha_{2} \cdots \alpha_{p}}
$$

with conformal dimension [384]

$$
\Delta=\frac{1}{2}\left(d \pm \sqrt{(d-2 p)^{2}+4 \mu^{2} R^{2}}\right)
$$

Thus the relation

$$
(\mu R)^{2}=(\Delta-p)(\Delta-d+p)
$$

for a $p$-form field with dimension $\Delta$. The relation (I.21) agrees with the conventions in Refs. [131, 385]. For a spinor field in AdS the mass-dimension relation is [386]

$$
\Delta=\frac{1}{2}(d+2|\mu R|)
$$

The relation for spin- $\frac{3}{2}$ is unchanged $[183,387]$. 


\section{Bibliography}

[1] M. Breidenbach et al., Observed behavior of highly inelastic electron-proton scattering, Phys. Rev. Lett. 23, 935 (1969).

[2] E. Perez and E. Rizvi, The quark and gluon structure of the proton, Rep. Prog. Phys. 76, 046201 (2013) [arXiv:1208.1178 [hep-ex]].

[3] W. J. Marciano and H. Pagels, Quantum chromodynamics: a review, Phys. Rept. 36, 137 (1978).

[4] N. Brambilla, S. Eidelman, P. Foka et al., QCD and strongly coupled gauge theories: challenges and perspectives, arXiv:1404.3723 [hep-ph].

[5] G. Parisi, Conformal invariance in perturbation theory, Phys. Lett. B 39, 643 (1972).

[6] V. M. Braun, G. P. Korchemsky and D. Mueller, The uses of conformal symmetry in QCD, Prog. Part. Nucl. Phys. 51 (2003) 311 [arXiv:hep-ph/0306057].

[7] K. G. Wilson, Confinement of quarks, Phys. Rev. D 10, 2445 (1974).

[8] Z. Fodor and C. Hoelbling, Light hadron masses from lattice QCD, Rev. Mod. Phys. 84, 449 (2012) [arXiv:1203.4789 [hep-lat]].

[9] J. M. Cornwall, Dynamical mass generation in continuum QCD, Phys. Rev. D 26, 1453 (1982).

[10] C. D. Roberts and A. G. Williams, Dyson-Schwinger equations and their application to hadronic physics, Prog. Part. Nucl. Phys. 33, 477 (1994) [arXiv:hep-ph/9403224]. 
[11] R. Alkofer and L. von Smekal, The infrared behavior of QCD Green's functions: Confinement dynamical symmetry breaking, and hadrons as relativistic bound states, Phys. Rept. 353, 281 (2001) [arXiv:hep-ph/0007355].

[12] C. S. Fischer, Infrared properties of QCD from Dyson-Schwinger equations, J. Phys. G 32, R253 (2006) [arXiv:hep-ph/0605173].

[13] T. Kinoshita, Mass singularities of Feynman amplitudes, J. Math. Phys. 3, 650 (1962).

[14] T. D. Lee and M. Nauenberg, Degenerate systems and mass singularities, Phys. Rev. 133, B1549 (1964).

[15] E. E. Salpeter and H. A. Bethe, A relativistic equation for bound state problems, Phys. Rev. 84, 1232 (1951).

[16] K. A. Olive et al. (Particle Data Group Collaboration), Review of Particle Physics, Chin. Phys. C 38, 090001 (2014).

[17] T. Regge, Introduction to complex orbital momenta, Nuovo Cim. 14, 951 (1959).

[18] T. Regge, Bound states, shadow states and Mandelstam representation, Nuovo Cim. 18, 947 (1960).

[19] P. D. B. Collins, An Introduction to Regge Theory and High-Energy Physics (Cambridge University Press, 1977).

[20] S. Donnachie, H. G. Dosch, O. Nachtmann and P. Landshoff, Pomeron physics and QCD, Camb. Monogr. Part. Phys. Nucl. Phys. Cosmol. 19, 1 (2002).

[21] P. A. M. Dirac, Forms of relativistic dynamics, Rev. Mod. Phys. 21, 392 (1949).

[22] J. M. Maldacena, The large-N limit of superconformal field theories and supergravity, Int. J. Theor. Phys. 38, 1113 (1999) [arXiv:hep-th/9711200].

[23] J. D. Bekenstein, Black holes and entropy, Phys. Rev. D 7, 2333 (1973).

[24] S. W. Hawking, Particle creation by black holes, Commun. Math. Phys. 43, 199 (1975).

[25] G. 't Hooft, Dimensional reduction in quantum gravity, [arXiv:gr-qc/9310026]. 
[26] L. Susskind, The world as a hologram, J. Math. Phys. 36, 6377 (1995) [arXiv:hep-th/9409089].

[27] S. S. Gubser, I. R. Klebanov and A. M. Polyakov, Gauge theory correlators from non-critical string theory, Phys. Lett. B 428, 105 (1998) [arXiv:hep-th/9802109].

[28] E. Witten, Anti-de Sitter space and holography, Adv. Theor. Math. Phys. 2, 253 (1998) [arXiv:hep-th/9802150].

[29] J. Polchinski, String Theory. Vol. 2: Superstring Theory and Beyond (Cambridge University Press, 1998).

[30] B. Lucini and M. Panero, $S U(N)$ gauge theories at large N, Phys. Rept. 526, 93 (2013) [arXiv:1210.4997 [hep-th]].

[31] G. Mack and A. Salam, Finite component field representations of the conformal group, Annals Phys. 53, 174 (1969).

[32] J. Polchinski and M. J. Strassler, The string dual of a confining four-dimensional gauge theory, arXiv:hep-th/0003136.

[33] I. R. Klebanov and M. J. Strassler, Supergravity and a confining gauge theory: duality cascades and $\chi$ SB-resolution of naked singularities, JHEP 0008, 052 (2000) [arXiv:hep-th/0007191].

[34] E. Witten, Anti-de Sitter space, thermal phase transition, and confinement in gauge theories, Adv. Theor. Math. Phys. 2, 505 (1998) [arXiv:hep-th/9803131].

[35] S. J. Rey, S. Theisen and J. T. Yee, Wilson-Polyakov loop at finite temperature in large-N gauge theory and anti-de Sitter supergravity, Nucl. Phys. B 527, 171 (1998) [arXiv:hep-th/9803135].

[36] A. Brandhuber, N. Itzhaki, J. Sonnenschein and S. Yankielowicz, Wilson loops in the large $\mathrm{N}$ limit at finite temperature, Phys. Lett. B 434, 36 (1998) [arXiv:hep-th/9803137].

[37] D. J. Gross and H. Ooguri, Aspects of large N gauge theory dynamics as seen by string theory, Phys. Rev. D 58, 106002 (1998) [arXiv:hep-th/9805129].

[38] T. Sakai and S. Sugimoto, Low energy hadron physics in holographic QCD, Prog. Theor. Phys. 113, 843 (2005) [arXiv:hep-th/0412141]. 
[39] T. Sakai and S. Sugimoto, More on a holographic dual of QCD, Prog. Theor. Phys. 114, 1083 (2005) [arXiv:hep-th/0507073].

[40] D. J. Gross and F. Wilczek, Ultraviolet behavior of nonabelian gauge theories, Phys. Rev. Lett. 30, 1343 (1973).

[41] H. D. Politzer, Reliable perturbative results for strong interactions?, Phys. Rev. Lett. 30, 1346 (1973).

[42] S. Furui and H. Nakajima, Infrared features of unquenched finite temperature lattice Landau gauge QCD, Phys. Rev. D 76, 054509 (2007) [arXiv:hep-lat/0612009].

[43] L. von Smekal, R. Alkofer and A. Hauck, The infrared behavior of gluon and ghost propagators in Landau gauge QCD, Phys. Rev. Lett. 79, 3591 (1997) [arXiv:hep-ph/9705242].

[44] For a recent review see for example: D. Binosi, J. Papavassiliou, Pinch technique: theory and applications, Phys. Rept. 479, 1 (2009) [arXiv:0909.2536 [hep-ph]], and references therein.

[45] A. Deur, V. Burkert, J. P. Chen and W. Korsch, Experimental determination of the effective strong coupling constant, Phys. Lett. B 650, 244 (2007) [arXiv:hep-ph/0509113].

[46] S. J. Brodsky and R. Shrock, Maximum wavelength of confined quarks and gluons and properties of quantum chromodynamics, Phys. Lett. B 666, 95 (2008) [arXiv:0806.1535 [hep-th]].

[47] S. J. Brodsky, G. F. de Teramond and A. Deur, Nonperturbative QCD coupling and its $\beta$-function from light-front holography, Phys. Rev. D 81, 096010 (2010) [arXiv: 1002.3948 [hep-ph]].

[48] A. Deur, S. J. Brodsky and G. F. de Teramond, "Scheme-independent determination of the perturbative QCD scale $\boldsymbol{\Lambda}_{\mathrm{s}}$ from confinement dynamics in holographic QCD," arXiv:1409.5488 [hep-ph].

[49] N. Isgur and J. E. Paton, A flux tube model for hadrons in QCD, Phys. Rev. D 31, $2910(1985)$.

[50] N. Isgur, R. Kokoski and J. E. Paton, Gluonic excitations of mesons: why they are missing and where to find them, Phys. Rev. Lett. 54, 869 (1985). 
[51] S. J. Brodsky and G. F. de Teramond, AdS/QCD, light-front holography, and sublimated gluons, PoS QCD -TNT-II (2011) 008 [arXiv:1112.4212 [hep-th]].

[52] J. D. Bjorken, Two topics in quantum chromodynamics, in Cargese Summer Institute in Particle Physics, Cargese, France, Jul 18 - Aug 4, 1989, SLAC-PUB-5103 (1989).

[53] M. Kruczenski, D. Mateos, R. C. Myers and D. J. Winters, Towards a holographic dual of large- $N_{C}$ QCD, JHEP 0405, 041 (2004) [arXiv:hep-th/0311270].

[54] D. Becciolini, M. Redi and A. Wulzer, AdS/QCD: The relevance of the geometry, JHEP 1001, 074 (2010) [arXiv:0906.4562 [hep-ph]].

[55] J. Polchinski and M. J. Strassler, Hard scattering and gauge/string duality, Phys. Rev. Lett. 88, 031601 (2002) [arXiv:hep-th/0109174].

[56] S. J. Brodsky and G. R. Farrar, Scaling laws at large transverse momentum, Phys. Rev. Lett. 31, 1153 (1973).

[57] S. J. Brodsky and G. R. Farrar, Scaling laws for large momentum transfer processes, Phys. Rev. D 11 (1975) 1309.

[58] V. A. Matveev, R. M. Muradian and A. N. Tavkhelidze, Automodellism in the large-angle elastic scattering and structure of hadrons, Lett. Nuovo Cim. 7, 719 (1973).

[59] E. Schreiber, Excited mesons and quantization of string endpoints, arXiv:hep-th/0403226.

[60] M. Shifman, Highly excited hadrons in QCD and beyond, arXiv:hep-ph/0507246.

[61] A. Karch, E. Katz, D. T. Son and M. A. Stephanov, Linear confinement and AdS/QCD, Phys. Rev. D 74, 015005 (2006) [arXiv:hep-ph/0602229].

[62] M. Shifman and A. Vainshtein, Highly excited mesons, linear Regge trajectories and the pattern of the chiral symmetry realization, Phys. Rev. D 77, 034002 (2008) [arXiv:0710.0863 [hep-ph]].

[63] C. Csaki and M. Reece, Toward a systematic holographic QCD: A braneless approach, JHEP 0705, 062 (2007) [arXiv:hep-ph/0608266]. 
[64] J. P. Shock, F. Wu, Y. -L. Wu and Z. -F. Xie, AdS/QCD phenomenological models from a back-reacted geometry, JHEP 0703, 064 (2007) [arXiv:hep-ph/0611227].

[65] U. Gursoy and E. Kiritsis, Exploring improved holographic theories for QCD: Part I, JHEP 0802, 032 (2008) [arXiv:0707.1324 [hep-th]].

[66] U. Gursoy, E. Kiritsis and F. Nitti, Exploring improved holographic theories for QCD: Part II, JHEP 0802, 019 (2008) [arXiv:0707.1349 [hep-th]].

[67] B. Batell and T. Gherghetta, Dynamical soft-wall AdS/QCD, Phys. Rev. D 78, 026002 (2008) [arXiv:0801.4383 [hep-ph]].

[68] W. de Paula, T. Frederico, H. Forkel and M. Beyer, Dynamical AdS/QCD with area-law confinement and linear Regge trajectories, Phys. Rev. D 79, 075019 (2009) [arXiv:0806.3830 [hep-ph]].

[69] J. Erlich, E. Katz, D. T. Son and M. A. Stephanov, QCD and a holographic model of hadrons, Phys. Rev. Lett. 95, 261602 (2005) [arXiv:hep-ph/0501128].

[70] L. Da Rold and A. Pomarol, Chiral symmetry breaking from five dimensional spaces, Nucl. Phys. B 721, 79 (2005) [arXiv:hep-ph/0501218].

[71] L. Da Rold and A. Pomarol, The scalar and pseudoscalar sector in a fivedimensional approach to chiral symmetry breaking, JHEP 0601, 157 (2006) [arXiv:hep-ph/0510268].

[72] S. J. Brodsky and G. F. de Teramond, Light-front hadron dynamics and AdS/CFT correspondence, Phys. Lett. B 582, 211 (2004) [arXiv:hep-th/0310227].

[73] S. J. Brodsky, H. C. Pauli and S. S. Pinsky, Quantum chromodynamics and other field theories on the light cone, Phys. Rept. 301, 299 (1998) [arXiv:hep-ph/9705477].

[74] G. F. de Teramond and S. J. Brodsky, Light-front holography: a first approximation to QCD, Phys. Rev. Lett. 102, 081601 (2009) [arXiv:0809.4899 [hep-ph]].

[75] S. J. Brodsky and G. F. de Teramond, Hadronic spectra and light-front wave functions in holographic QCD, Phys. Rev. Lett. 96, 201601 (2006) [arXiv: hep-ph/0602252]. 
[76] S. J. Brodsky and G. F. de Teramond, Light-front dynamics and AdS/QCD correspondence: the pion form factor in the space- and time-like regions, Phys. Rev. D 77, 056007 (2008) [arXiv:0707.3859 [hep-ph]].

[77] J. Polchinski and M. J. Strassler, Deep inelastic scattering and gauge/string duality, JHEP 0305, 012 (2003) [arXiv:hep-th/0209211].

[78] S. D. Drell and T. M. Yan, Connection of elastic electromagnetic nucleon formfactors at large $Q^{2}$ and deep inelastic structure functions near threshold, Phys. Rev. Lett. 24, 181 (1970).

[79] G. B. West, Phenomenological model for the electromagnetic structure of the proton, Phys. Rev. Lett. 24, 1206 (1970).

[80] S. J. Brodsky and S. D. Drell, The anomalous magnetic moment and limits on fermion substructure, Phys. Rev. D 22, 2236 (1980).

[81] S. J. Brodsky and G. F. de Teramond, Light-front dynamics and AdS/QCD correspondence: gravitational form factors of composite hadrons, Phys. Rev. D 78, 025032 (2008) [arXiv:0804.0452 [hep-ph]].

[82] Z. Abidin and C. E. Carlson, Gravitational form factors of vector mesons in an AdS/QCD model, Phys. Rev. D 77, 095007 (2008) [arXiv:0801.3839 [hep-ph]].

[83] C. Fronsdal, Singletons and massless, integral-spin fields on de Sitter space, Phys. Rev. D20, 848 (1979).

[84] E. S. Fradkin and M. A. Vasiliev, Cubic interaction in extended theories of massless higher-spin fields, Nucl. Phys. B 291, 141 (1987).

[85] I. L. Buchbinder, A. Pashnev and M. Tsulaia, Lagrangian formulation of the massless higher integer spin fields in the AdS background, Phys. Lett. B 523, 338 (2001) [arXiv:hep-th/0109067].

[86] R. R. Metsaev, Light-cone form of field dynamics in anti-de Sitter spacetime and AdS/CFT correspondence, Nucl. Phys. B 563, 295 (1999) [arXiv:hep-th/9906217].

[87] R. R. Metsaev, Massive totally symmetric fields in $\mathrm{AdS}_{d}$, Phys. Lett. B 590, 95 (2004) [arXiv:hep-th/0312297]. 
[88] R. R. Metsaev, Anomalous conformal currents, shadow fields and massive AdS fields, Phys. Rev. D 85, 126011 (2012) [arXiv:1110.3749 [hep-th]].

[89] R. R. Metsaev, Light-cone gauge approach to arbitrary spin fields, currents, and shadows, J. Phys. A 47, 375401 (2014). [arXiv:1312.5679 [hep-th]].

[90] R. R. Metsaev, Arbitrary spin conformal fields in (A)dS, Nucl. Phys. B 885, 734 (2014) [arXiv:1404.3712 [hep-th]].

[91] G. F. de Teramond, H. G. Dosch and S. J. Brodsky, Kinematical and dynamical aspects of higher-spin bound-state equations in holographic QCD, Phys. Rev. D 87, 075005 (2013) [arXiv:1301.1651 [hep-ph]].

[92] H. C. Pauli and S. J. Brodsky, Solving field theory in one space one time dimension, Phys. Rev. D 32, 1993 (1985).

[93] H. C. Pauli and S. J. Brodsky, Discretized light cone quantization: solution to a field theory in one space one time dimensions, Phys. Rev. D 32, 2001 (1985).

[94] V. de Alfaro, S. Fubini and G. Furlan, Conformal invariance in quantum mechanics, Nuovo Cim. A 34, 569 (1976).

[95] S. J. Brodsky, G. F. de Teramond and H. G. Dosch, Threefold complementary approach to holographic QCD, Phys. Lett. B 729, 3 (2014) [arXiv:1302.4105 [hep-th]].

[96] H. Leutwyler and J. Stern, Harmonic confinement: A fully relativistic approximation to the meson spectrum, Phys. Lett. B 73, 75 (1978).

[97] A. P. Trawiński, S. D. Glazek, S. J. Brodsky, G. F. de Teramond and H. G. Dosch, Effective confining potentials for QCD, Phys. Rev. D 90, 074017 (2014) [arXiv:1403.5651 [hep-ph]].

[98] E. Eichten, K. Gottfried, T. Kinoshita, K. D. Lane and T. -M. Yan, Charmonium: The model, Phys. Rev. D 17, 3090 (1978).

[99] J. Erlich, G. D. Kribs and I. Low, Emerging holography, Phys. Rev. D 73, 096001 (2006) [arXiv:hep-th/0602110].

[100] A. A. Migdal, Multicolor QCD as dual resonance theory, Annals Phys. 109, 365 (1977). 
[101] A. A. Migdal, Series expansion for mesonic masses in multicolor QCD, Annals Phys. 110, 46 (1978).

[102] M. A. Shifman, A. I. Vainshtein and V. I. Zakharov, QCD and resonance physics. Sum rules, Nucl. Phys. B 147, 385 (1979).

[103] O. Cata, Towards understanding Regge trajectories in holographic QCD, Phys. Rev. D 75, 106004 (2007) [arXiv:hep-ph/0605251].

[104] F. Jugeau, S. Narison and H. Ratsimbarison, SVZ $\oplus 1 / \mathrm{q}^{2}$-expansion versus some QCD holographic models, Phys. Lett. B 722, 111 (2013) [arXiv:1302.6909 [hep-ph]].

[105] R. de Mello Koch, A. Jevicki, K. Jin and J. P. Rodrigues, AdS $4 / \mathrm{CFT}_{3}$ construction from collective fields, Phys. Rev. D 83, 025006 (2011) [arXiv: 1008.0633 [hep-th]].

[106] A. Jevicki, K. Jin and J. Yoon, $1 / N$ and loop corrections in higher spin $\mathrm{AdS}_{4} / \mathrm{CFT}_{3}$ duality, Phys. Rev. D 89, 085039 (2014) [arXiv:1401.3318 [hep-th]].

[107] R. de Mello Koch, A. Jevicki, J. P. Rodrigues and J. Yoon, Holography as a gauge phenomenon in higher spin duality, JHEP 1501, 055 (2015) [arXiv:1408.1255 [hep-th]].

[108] R. de Mello Koch, A. Jevicki, J. P. Rodrigues and J. Yoon, Canonical formulation of $O(N)$ vector/higher spin correspondence, arXiv:1408.4800 [hep-th].

[109] E. Mintun and J. Polchinski, Higher spin holography, RG, and the light cone," arXiv:1411.3151 [hep-th].

[110] S. D. Glazek and A. P. Trawiński, Model of the AdS/QFT duality, Phys. Rev. D 88, 105025 (2013) [arXiv:1307.2059 [hep-ph]].

[111] X. -L. Qi, Exact holographic mapping and emergent space-time geometry, arXiv:1309.6282 [hep-th].

[112] D. D. Dietrich, Hadrons on the worldline, holography, and Wilson flow, Phys. Rev. D 89, 086005 (2014) [arXiv:1312.5718 [hep-ph]].

[113] J. Erlich and C. Westenberger, Tests of universality in AdS/QCD, Phys. Rev. D 79, 066014 (2009) [arXiv:0812.5105 [hep-ph]]. 
[114] J. Erlich, How well does AdS/QCD describe QCD?, Int. J. Mod. Phys. A 25, 411 (2010) [arXiv:0908.0312 [hep-ph]].

[115] C. Csaki, M. Reece and J. Terning, The AdS/QCD correspondence: still undelivered, JHEP 0905, 067 (2009) [arXiv:0811.3001 [hep-ph]].

[116] J. R. Forshaw and D. A. Ross, Quantum chromodynamics and the pomeron, (Cambridge University Press, 1997).

[117] R. C. Brower, J. Polchinski, M. J. Strassler and C. -I Tan, The Pomeron and gauge/string duality, JHEP 0712, 005 (2007) [arXiv:hep-th/0603115].

[118] R. C. Brower, M. Djuric and C. -I Tan, Odderon in gauge/string duality, JHEP 0907, 063 (2009) [arXiv:0812.0354 [hep-th]].

[119] S. K. Domokos, J. A. Harvey and N. Mann, Pomeron contribution to $p p$ and $p \bar{p}$ scattering in AdS/QCD, Phys. Rev. D 80, 126015 (2009) [arXiv:0907.1084 [hep-ph]].

[120] S. K. Domokos, J. A. Harvey and N. Mann, Setting the scale of the $p p$ and $p \bar{p}$ total cross sections using AdS/QCD, Phys. Rev. D 82, 106007 (2010) [arXiv:1008. 2963 [hep-th]].

[121] R. Nishio and T. Watari, High-energy photon-hadron scattering in holographic QCD, Phys. Rev. D 84, 075025 (2011) [arXiv:1105.2999 [hep-ph]].

[122] J. -H. Gao and B. -W. Xiao, Nonforward Compton scattering in AdS/CFT correspondence, Phys. Rev. D 81, 035008 (2010) [arXiv:0912.4333 [hep-ph]].

[123] C. Marquet, C. Roiesnel and S. Wallon, Virtual compton scattering off a spinless target in AdS/QCD, JHEP 1004, 051 (2010) [arXiv:1002.0566 [hep-ph]].

[124] M. S. Costa and M. Djuric, Deeply virtual compton scattering from gauge/gravity duality, Phys. Rev. D 86, 016009 (2012) [arXiv:1201.1307 [hep-th]].

[125] J. -H. Gao and B. -W. Xiao, Polarized deep inelastic and elastic scattering from gauge/string duality, Phys. Rev. D 80, 015025 (2009) [arXiv:0904.2870 [hep-ph]]. 
[126] J. Casalderrey-Solana, H. Liu, D. Mateos, K. Rajagopal and U. A. Wiedemann, Gauge/string duality, hot QCD and heavy ion collisions, arXiv:1101.0618 [hep-th].

[127] J. de Boer, E. P. Verlinde and H. L. Verlinde, On the holographic renormalization group, JHEP 0008, 003 (2000) [arXiv:hep-th/9912012].

[128] K. G. Wilson and J. B. Kogut, The renormalization group and the epsilon expansion, Phys. Rept. 12, 75 (1974).

[129] K. Skenderis, Lecture notes on holographic renormalization, Class. Quant. Grav. 19, 5849 (2002) [arXiv:hep-th/0209067].

[130] M. Bianchi, D. Z. Freedman and K. Skenderis, Holographic renormalization, Nucl. Phys. B 631, 159 (2002)] [arXiv:hep-th/0112119].

[131] O. Aharony, S. S. Gubser, J. M. Maldacena, H. Ooguri and Y. Oz, Large $N$ field theories, string theory and gravity, Phys. Rept. 323, 183 (2000) [arXiv:hep-th/9905111].

[132] I. R. Klebanov, TASI lectures: Introduction to the AdS/CFT correspondence, arXiv:hep-th/0009139.

[133] E. D'Hoker and D. Z. Freedman, Supersymmetric gauge theories and the AdS/CFT correspondence, arXiv:hep-th/0201253.

[134] J. Erdmenger, N. Evans, I. Kirsch and E. Threlfall, Mesons in gauge/gravity duals, Eur. Phys. J. A 35, 81 (2008) [arXiv:0711.4467 [hep-th]]

[135] K. Peeters and M. Zamaklar, The string/gauge theory correspondence in QCD, Eur. Phys. J. ST 152, 113 (2007) [arXiv:0708.1502 [hep-ph]].

[136] Y. Kim, I. J. Shin and T. Tsukioka, Holographic QCD: Past, present, and future, Prog. Part. Nucl. Phys. 68, 55 (2013) [arXiv:1205.4852 [hep-ph]].

[137] A. V. Ramallo, Introduction to the AdS/CFT correspondence, [arXiv:1310.4319 [hep-th].

[138] S. J. Brodsky and G. F. de Teramond, AdS/CFT and light-front QCD, in Search for the Totally Unexpected in the LHC Era, Proceedings of the International 
School of Subnuclear Physics, Vol. 45 (World Scientific Publishing Co., 2009), arXiv:0802.0514 [hep-ph].

[139] R. P. Feynman, Very high-energy collisions of hadrons, Phys. Rev. Lett. 23, 1415 (1969).

[140] R. P. Feynman, Photon-hadron interactions, (Benjamin, 1972).

[141] S. Fubini and G. Furlan, Renormalization effects for partially conserved currents, Physics 1, 229 (1965).

[142] S. Weinberg, Dynamics at infinite momentum, Phys. Rev. 150, 1313 (1966).

[143] J. D. Bjorken, Asymptotic sum rules at infinite momentum, Phys. Rev. 179, 1547 (1969).

[144] M. Gell-Mann, A schematic model of baryons and mesons, Phys. Lett. 8, 214 (1964).

[145] G. Zweig, An SU(3) model for strong interaction symmetry and its breaking, I and II, CERN-TH-401 and 412 (1964).

[146] S. Godfrey and N. Isgur, Mesons in a relativized quark model with chromodynamics, Phys. Rev. D 32, 189 (1985).

[147] H. Fritzsch and M. Gell-Mann, Current algebra: Quarks and what else?, in Proc. XVI Int. Conf. on High Energy Phys. eds. J. D. Jackson and A. Roberts (Fermilab, 1972) Vol. 2, p.135 [arXiv:hep-ph/0208010].

[148] H. Fritzsch, M. Gell-Mann and H. Leutwyler, Advantages of the color octet gluon picture, Phys. Lett. B 47, 365 (1973).

[149] A. Casher and L. Susskind, A quark model of mesons based on chiral symmetry, Phys. Lett. B 44, 171 (1973).

[150] K. G. Wilson, T. S. Walhout, A. Harindranath, W. -M. Zhang, R. J. Perry and S. D. Glazek, Nonperturbative QCD: A weak coupling treatment on the light front, Phys. Rev. D 49, 6720 (1994) [arXiv:hep-th/9401153].

[151] W. A. Bardeen and R. B. Pearson, Local gauge invariance and the bound state nature of hadrons, Phys. Rev. D 14, 547 (1976). 
[152] R. F. Streater and A. S. Wightman, PCT, spin and statistics, and all that (Princeton University Press, 1964).

[153] H. Leutwyler and J. Stern, Relativistic dynamics on a null plane, Annals Phys. 112, 94 (1978).

[154] J. B. Kogut and D. E. Soper, Quantum electrodynamics in the infinite momentum frame, Phys. Rev. D 1, 2901 (1970).

[155] P. P. Srivastava, Perspectives of light front quantized field theory: Some new results, in Quantum Field Theory, p. 437-478, edited by A. N. Mitra [arXiv:hep-ph/9908492].

[156] S. J. Brodsky and R. Shrock, Condensates in quantum chromodynamics and the cosmological constant, Proc. Nat. Acad. Sci. 108, 45 (2011) [arXiv:0905.1151 [hep-th]].

[157] S. J. Brodsky, C. D. Roberts, R. Shrock and P. C. Tandy, Confinement contains condensates, Phys. Rev. C 85, 065202 (2012) [arXiv:1202.2376 [nucl-th]].

[158] M. Burkardt, Light front quantization, Adv. Nucl. Phys. 23, 1 (1996) [arXiv:hep-ph/9505259].

[159] G. P. Lepage and S. J. Brodsky, Exclusive processes in perturbative quantum chromodynamics, Phys. Rev. D 22, 2157 (1980).

[160] S. J. Brodsky, D. S. Hwang, B. Q. Ma and I. Schmidt, Light-cone representation of the spin and orbital angular momentum of relativistic composite systems, Nucl. Phys. B 593, 311 (2001) [arXiv:hep-th/0003082].

[161] H. C. Pauli, On confinement in a light cone Hamiltonian for QCD, Eur. Phys. J. C 7, 289 (1999) [arXiv:hep-th/9809005].

[162] G. E. Brown, T. T. S. Kuo, J. W. Holt and S. Lee, The Nucleon-Nucleon Interaction and the Nuclear Many-Body Problem (World Scientific Publishing Co., 2010).

[163] T. Appelquist, M. Dine, The static potential in quantum chromodynamics, Phys. Lett. B 69, 231 (1977). 
[164] T. Appelquist, M. Dine and I. J. Muzinich, The static limit of quantum chromodynamics, Phys. Rev. D 17, 2074 (1978).

[165] S. S. Chabysheva and J. R. Hiller, A light-front coupled-cluster method for the nonperturbative solution of quantum field theories, Phys. Lett. B 711, 417 (2012) [arXiv: 1103.0037 [hep-ph]].

[166] F. Antonuccio, S. J. Brodsky and S. Dalley, Light-cone wavefunctions at small $x$, Phys. Lett. B 412, 104 (1997) [arXiv:hep-ph/9705413].

[167] D. E. Soper, The parton model and the Bethe-Salpeter wave function, Phys. Rev. D 15, 1141 (1977).

[168] L. D. Landau and E. M. Lifshitz, Quantum Mechanics (Pergamon Press, 1958) Sec. 35 .

[169] T. Gutsche, V. E. Lyubovitskij, I. Schmidt and A. Vega, Light-front potential for heavy quarkonia constrained by the holographic soft-wall model, Phys. Rev. D 90, 096007 (2014) [arXiv:1410.3738 [hep-ph]].

[170] W. I. Weisberger, Partons, electromagnetic mass shifts, and the approach to scaling, Phys. Rev. D 5, 2600 (1972).

[171] S. S. Chabysheva and J. R. Hiller, Dynamical model for longitudinal wave functions in light-front holographic QCD, Annals Phys. 337, 143 (2013) [arXiv:1207.7128 [hep-ph]].

[172] S. Fubini and E. Rabinovici, Superconformal Quantum Mechanics, Nucl. Phys. B 245, 17 (1984).

[173] C. Chamon, R. Jackiw, S. -Y. Pi and L. Santos, Conformal quantum mechanics as the $\mathrm{CFT}_{1}$ dual to $\mathrm{AdS}_{2}$, Phys. Lett. B 701, 503 (2011) [arXiv:1106.0726 [hep-th]].

[174] D. Kharzeev, E. Levin and K. Tuchin, Broken scale invariance, massless dilaton and confinement in QCD, JHEP 0906, 055 (2009) [arXiv:0809.3794 [hep-ph]].

[175] W. Muck and K. S. Viswanathan, Conformal field theory correlators from classical field theory on anti-de Sitter space. II: Vector and spinor fields, Phys. Rev. D 58, 106006 (1998) [arXiv:hep-th/9805145]. 
[176] G. F. de Teramond and S. J. Brodsky, Hadronic form factor models and spectroscopy within the gauge/gravity correspondence, arXiv:1203.4025 [hep-ph].

[177] J. Sonnenschein, Stringy confining Wilson loops, arXiv:hep-th/0009146.

[178] I. R. Klebanov and J. M. Maldacena, Solving quantum field theories via curved spacetimes, Phys. Today 62, 28 (2009).

[179] W. Rarita and J. Schwinger, On a theory of particles with half integral spin, Phys. Rev. 60, 61 (1941).

[180] Z. Abidin and C. E. Carlson, Nucleon electromagnetic and gravitational form factors from holography, Phys. Rev. D 79, 115003 (2009) [arXiv:0903.4818 [hep-ph]].

[181] T. Gutsche, V. E. Lyubovitskij, I. Schmidt and A. Vega, Dilaton in a soft-wall holographic approach to mesons and baryons, Phys. Rev. D 85, 076003 (2012) [arXiv:1108.0346 [hep-ph]].

[182] I. Kirsch, Spectroscopy of fermionic operators in AdS/CFT, JHEP 0609, 052 (2006) [arXiv:hep-th/0607205].

[183] A. Volovich, Rarita-Schwinger field in the AdS / CFT correspondence, JHEP 9809, 022 (1998) [arXiv:hep-th/9809009].

[184] P. Matlock and K. S. Viswanathan, The AdS/CFT correspondence for the massive Rarita-Schwinger field, Phys. Rev. D 61, 026002 (2000) [arXiv:hep-th/9906077].

[185] See, for example: J. J. Dudek, R. G. Edwards, K. Orginos and D. G. Richards, Lattice QCD and the Jefferson Lab program, J. Phys. Conf. Ser. 299, 012007 (2011).

[186] See, for example: R. G. Edwards, J. J. Dudek, D. G. Richards and S. J. Wallace, Excited state baryon spectroscopy from lattice QCD, Phys. Rev. D 84, 074508 (2011) [arXiv:1104.5152 [hep-ph]].

[187] E. Witten, Baryons and branes in anti-de Sitter space, JHEP 9807, 006 (1998) [arXiv:hep-th/9805112].

[188] K. Nawa, H. Suganuma and T. Kojo, Baryons in holographic QCD, Phys. Rev. D 75, 086003 (2007) [arXiv:hep-th/0612187]. 
[189] D. K. Hong, M. Rho, H. -U. Yee and P. Yi, Chiral dynamics of baryons from string theory, Phys. Rev. D 76, 061901 (2007) [arXiv:hep-th/0701276].

[190] H. Hata, T. Sakai, S. Sugimoto and S. Yamato, Baryons from instantons in holographic QCD, Prog. Theor. Phys. 117, 1157 (2007) [arXiv:hep-th/0701280].

[191] G. F. de Teramond and S. J. Brodsky, Gauge/Gravity duality and hadron physics at the light front, AIP Conf. Proc. 1296, 128 (2010) [arXiv:1006.2431 [hep-ph]].

[192] P. Breitenlohner and D. Z. Freedman, Stability in gauged extended supergravity, Annals Phys. 144, 249 (1982).

[193] H. Boschi-Filho and N. R. F. Braga, QCD/string holographic mapping and glueball mass spectrum, Eur. Phys. J. C 32, 529 (2004) [arXiv:hep-th/0209080].

[194] H. Boschi-Filho and N. R. F. Braga, Gauge/string duality and scalar glueball mass ratios, JHEP 0305, 009 (2003) [arXiv:hep-th/0212207].

[195] C. J. Morningstar and M. J. Peardon, Efficient glueball simulations on anisotropic lattices, Phys. Rev. D 56, 4043 (1997) [arXiv:hep-lat/9704011].

[196] M. J. Teper, Physics from the lattice: Glueballs in QCD: Topology: SU(N) for all $\mathrm{N},[\operatorname{arXiv}:$ hep-lat/9711011].

[197] C. Csaki, H. Ooguri, Y. Oz and J. Terning, Glueball mass spectrum from supergravity, JHEP 9901, 017 (1999) [arXiv:hep-th/9806021].

[198] R. de Mello Koch, A. Jevicki, M. Mihailescu and J. P. Nunes, Evaluation of glueball masses from supergravity, Phys. Rev. D 58, 105009 (1998) [arXiv:hep-th/9806125].

[199] R. C. Brower, S. D. Mathur and C. -I Tan, Glueball spectrum for QCD from AdS supergravity duality, Nucl. Phys. B 587, 249 (2000) [arXiv:hep-th/0003115].

[200] G. F. de Teramond, S. J. Brodsky, Hadronic spectrum of a holographic dual of QCD, Phys. Rev. Lett. 94, 201601 (2005) [arXiv:hep-th/0501022].

[201] A. Chodos, R. L. Jaffe, K. Johnson, C. B. Thorn and V. F. Weisskopf, A new extended model of hadrons, Phys. Rev. D 9, 3471 (1974). 
[202] S. S. Afonin, Towards understanding broad degeneracy in non-strange mesons, Mod. Phys. Lett. A 22, 1359 (2007) [arXiv:hep-ph/0701089].

[203] E. Klempt and A. Zaitsev, Glueballs, hybrids, multiquarks: Experimental facts versus QCD inspired concepts, Phys. Rept. 454, 1 (2007) [arXiv:0708.4016 [hep-ph]].

[204] S. Weinberg, Precise relations between the spectra of vector and axial vector mesons, Phys. Rev. Lett. 18, 507 (1967).

[205] S. R. Beane, Broken chiral symmetry on a null plane, Annals Phys. 337, 111 (2013) [arXiv:1302.1600 [nucl-th]].

[206] Y. Nambu, QCD and the string model, Phys. Lett. B 80, 372 (1979).

[207] M. Baker and R. Steinke, Semiclassical quantization of effective string theory and Regge trajectories, Phys. Rev. D 65, 094042 (2002) [arXiv:hep-th/0201169].

[208] D. Parganlija, Mesons, PANDA and the scalar glueball, J. Phys. Conf. Ser. 503, 012010 (2014) [arXiv:1312.2830 [hep-ph]].

[209] E. Klempt and J. M. Richard, Baryon spectroscopy, Rev. Mod. Phys. 82, 1095 (2010) [arXiv:0901.205 [hep-ph]].

[210] S. Paul [COMPASS Collaboration], Precision spectroscopy with COMPASS and the observation of a new iso-vector resonance, EPJ Web Conf. 73, 03002 (2014) [arXiv:1312.3678 [hep-ex]].

[211] H. Boschi-Filho, N. R. F. Braga and H. L. Carrion, Glueball Regge trajectories from gauge/string duality and the Pomeron, Phys. Rev. D 73, 047901 (2006) [arXiv:hep-th/0507063].

[212] E. Katz, A. Lewandowski and M. D. Schwartz, Tensor mesons in AdS/QCD, Phys. Rev. D 74, 086004 (2006) [arXiv:hep-ph/0510388].

[213] N. Evans and A. Tedder, Perfecting the ultra-violet of holographic descriptions of QCD, Phys. Lett. B 642, 546 (2006) [arXiv:hep-ph/0609112].

[214] D. K. Hong, T. Inami and H. U. Yee, Baryons in AdS/QCD, Phys. Lett. B 646, 165 (2007) [arXiv:hep-ph/0609270]. 
[215] P. Colangelo, F. De Fazio, F. Jugeau and S. Nicotri, On the light glueball spectrum in a holographic description of QCD, Phys. Lett. B 652, 73 (2007) [arXiv:hep-ph/0703316].

[216] H. Forkel, M. Beyer and T. Frederico, Linear square-mass trajectories of radially and orbitally excited hadrons in holographic QCD, JHEP 0707, 077 (2007) [arXiv:0705.1857 [hep-ph]].

[217] H. Forkel, Holographic glueball structure, Phys. Rev. D 78, 025001 (2008) [arXiv:0711.1179 [hep-ph]].

[218] A. Vega and I. Schmidt, Scalar hadrons in $\mathrm{AdS}_{5} \times S^{5}$, Phys. Rev. D 78, 017703 (2008) [arXiv:0806.2267 [hep-ph]].

[219] K. Nawa, H. Suganuma and T. Kojo, Baryons with holography, Mod. Phys. Lett. A 23, 2364 (2008) [arXiv:0806.3040 [hep-th]].

[220] P. Colangelo, F. De Fazio, F. Giannuzzi, F. Jugeau and S. Nicotri, Light scalar mesons in the soft-wall model of AdS/QCD, Phys. Rev. D 78, 055009 (2008) [arXiv:0807.1054 [hep-ph]].

[221] H. Forkel and E. Klempt, Diquark correlations in baryon spectroscopy and holographic QCD, Phys. Lett. B 679, 77 (2009) [arXiv:0810.2959 [hep-ph]].

[222] T. Gherghetta, J. I. Kapusta and T. M. Kelley, Chiral symmetry breaking in the soft-wall AdS/QCD model, Phys. Rev. D 79, 076003 (2009) [arXiv:0902.1998 [hep-ph]].

[223] H. C. Ahn, D. K. Hong, C. Park and S. Siwach, Spin 3/2 baryons and form factors in AdS/QCD, Phys. Rev. D 80, 054001 (2009) [arXiv:0904.3731 [hep-ph]].

[224] W. de Paula and T. Frederico, Scalar mesons within a dynamical holographic QCD model, Phys. Lett. B 693 (2010) 287 [arXiv:0908.4282 [hep-ph]].

[225] Y. -Q. Sui, Y. -L. Wu, Z. -F. Xie and Y. -B. Yang, Prediction for the mass spectra of resonance mesons in the soft-wall AdS/QCD with a modified 5D metric, Phys. Rev. D 81, 014024 (2010) [arXiv:0909.3887 [hep-ph]].

[226] J. I. Kapusta and T. Springer, Potentials for soft wall AdS/QCD, Phys. Rev. D 81, 086009 (2010) [arXiv:1001.4799 [hep-ph]]. 
[227] P. Zhang, Improving the excited nucleon spectrum in hard-wall AdS/QCD, Phys. Rev. D 81, 114029 (2010) [arXiv:1002.4352 [hep-ph]].

[228] P. Zhang, Mesons and nucleons in soft-wall AdS/QCD, Phys. Rev. D 82, 094013 (2010) [arXiv:1007.2163 [hep-ph]].

[229] M. Kirchbach, C. B. Compean, Conformal symmetry and light flavor baryon spectra, Phys. Rev. D 82, 034008 (2010) [arXiv:1003.1747 [hep-ph]].

[230] I. Iatrakis, E. Kiritsis and A. Paredes, An AdS/QCD model from Sen's tachyon action, Phys. Rev. D 81, 115004 (2010) [arXiv:1003.2377 [hep-ph]].

[231] T. Branz, T. Gutsche, V. E. Lyubovitskij, I. Schmidt and A. Vega, Light and heavy mesons in a soft-wall holographic approach, Phys. Rev. D 82, 074022 (2010) [arXiv: 1008.0268 [hep-ph]].

[232] T. M. Kelley, S. P. Bartz and J. I. Kapusta, Pseudoscalar mass spectrum in a soft-wall model of AdS/QCD, Phys. Rev. D 83, 016002 (2011) [arXiv:1009.3009 [hep-ph]].

[233] Y. -Q. Sui, Y. -L. Wu and Y. -B. Yang, Predictive AdS/QCD model for mass spectra of mesons with three flavors, Phys. Rev. D 83, 065030 (2011) [arXiv: 1012.3518 $[\mathrm{hep}-\mathrm{ph}]$.

[234] S. S. Afonin, Low-energy holographic models for QCD, Phys. Rev. C 83, 048202 (2011) [arXiv:1102.0156 [hep-ph]].

[235] Y. -L. He and P. Zhang, Constraining the infrared behavior of the soft-wall AdS/QCD model, Phys. Rev. D 85, 094021 (2012) [arXiv:1105.6293 [hep-ph]].

[236] S. Liu and P. Zhang, Mesons and nucleons in the soft-wall AdS/QCD with constrained infrared background, Phys. Rev. D 86, 014015 (2012) [arXiv:1205.0485 [hep-ph]].

[237] E. F. Capossoli and H. Boschi-Filho, Odd spin glueball masses and the Odderon Regge trajectories from the holographic hardwall model, Phys. Rev. D 88, 026010 (2013) [arXiv:1301.4457 [hep-th]].

[238] D. Li and M. Huang, Dynamical holographic QCD model for glueball and light meson spectra, JHEP 1311, 088 (2013) [arXiv:1303.6929 [hep-ph]]. 
[239] S. S. Afonin and I. V. Pusenkov, The quark masses and meson spectrum: A holographic approach, Phys. Lett. B 726, 283 (2013) [arXiv:1306.3948 [hep-ph]].

[240] Y. Kim and D. Yi, Holography at work for nuclear and hadron physics, Adv. High Energy Phys. 2011, 259025 (2011) [arXiv:1107.0155 [hep-ph]].

[241] P. Colangelo, F. Giannuzzi and S. Nicotri, Holographic approach to finite temperature QCD: The case of scalar glueballs and scalar mesons, Phys. Rev. D 80, 094019 (2009) [arXiv:0909.1534 [hep-ph]].

[242] Z. Li and B. -Q. Ma, Baryon spectrum in a finite-temperature AdS/QCD model, Phys. Rev. D 89, 015014 (2014) [arXiv:1312.3451 [hep-ph]].

[243] L. A. H. Mamani, A. S. Miranda, H. Boschi-Filho and N. R. F. Braga, Vector meson quasinormal modes in a finite-temperature AdS/QCD model, JHEP 1403, 058 (2014) [arXiv:1312.3815 [hep-th]].

[244] C. Park, D. -Y. Gwak, B. -H. Lee, Y. Ko and S. Shin, Soft wall model in the hadronic medium, Phys. Rev. D 84, 046007 (2011) [arXiv:1104.4182 [hep-th]].

[245] S. J. Brodsky, T. Huang and G. P. Lepage, The hadronic wave function in quantum chromodynamics, SLAC-PUB-2540.

[246] J. R. Forshaw and R. Sandapen, An AdS/QCD holographic wavefunction for the rho meson and diffractive rho meson electroproduction, Phys. Rev. Lett. 109, 081601 (2012) [arXiv:1203.6088 [hep-ph]].

[247] M. Ahmady and R. Sandapen, Predicting $\bar{B}^{0} \rightarrow \rho^{0} \gamma$ and ${\overline{B_{s}}}^{0} \rightarrow \rho^{0} \gamma$ using holographic AdS/QCD distribution amplitudes for the $\rho$ meson, Phys. Rev. D 87, 054013 (2013) [arXiv:1212.4074 [hep-ph]].

[248] M. Ahmady and R. Sandapen, Predicting the isospin asymmetry in $B \rightarrow K^{*} \gamma$ using holographic AdS/QCD distribution amplitudes for the $K^{*}$, Phys. Rev. D 88, 014042 (2013) [arXiv: 1305.1479 [hep-ph]].

[249] M. Ahmady, R. Campbell, S. Lord and R. Sandapen, Predicting the $B \rightarrow \rho$ form factors using AdS/QCD distribution amplitudes for the $\rho$ meson, Phys. Rev. D 88, 074031 (2013) [arXiv:1308.3694 [hep-ph]]. 
[250] M. Ahmady, R. Campbell, S. Lord and R. Sandapen, Predicting $B \rightarrow K^{*}$ form factors in light-cone QCD, Phys. Rev. D 88, 014042 (2014) [arXiv:1401.6707 [hep-ph]].

[251] M. R. Ahmady, S. Lord and R. Sandapen, Isospin asymmetry in $B \rightarrow K^{*} \mu^{+} \mu^{-}$ using AdS/QCD, Phys. Rev. D 90, 074010 (2014) [arXiv:1407.6700 [hep-ph]].

[252] C. -W. Hwang, Meson distribution amplitudes in holographic models, Phys. Rev. D 86, 014005 (2012) [arXiv:1204.1791 [hep-ph]].

[253] G. F. de Teramond, Light-front holography and the hadronic spectrum, Invited Talk at the Third Workshop of the APS Topical Group in Hadron Physics GHP2009, Denver, Colorado, April 2009.

[254] H. G. Dosch, S. J. Brodsky and G. F. de Teramond, Modified anti-de-Sitter metric, light-front quantized QCD, and conformal quantum mechanics, arXiv:1401.7710 [hep-ph].

[255] T. Gutsche, V. E. Lyubovitskij, I. Schmidt and A. Vega, Chiral symmetry breaking and meson wave functions in soft-wall AdS/QCD, Phys. Rev. D 87, 056001 (2013) [arXiv:1212.5196 [hep-ph]].

[256] M. Fujita, T. Kikuchi, K. Fukushima, T. Misumi and M. Murata, Melting spectral functions of the scalar and vector mesons in a holographic QCD model, Phys. Rev. D 81, 065024 (2010) [arXiv:0911.2298 [hep-ph]].

[257] G. F. de Teramond, AdS/CFT Integrability and light-front dynamics in a semiclassical approximation to QCD, Invited Talks at 11th International Conference, Baryons' 07, Seoul, Korea, June 2007 and Exploring QCD: Deconfinement, Extreme Environments and Holography, Isaac Newton Institute for Mathematical Sciences, Cambridge, UK, August 2007.

[258] W. Mueck and K. S. Viswanathan, Conformal field theory correlators from classical field theory on anti-de Sitter space: Vector and spinor fields, Phys. Rev. D 58, 106006 (1998) [arXiv:hep-th/9805145].

[259] A. V. Anisovich, R. Beck, E. Klempt, V. A. Nikonov, A. V. Sarantsev and U. Thoma, Properties of baryon resonances from a multichannel partial wave analysis, arXiv:1112.4937 [hep-ph]. 
[260] D. B. Lichtenberg, Unitary Symmetry and Elementary Particles (Academic Press, 1978).

[261] L. Y. Glozman, "Parity doublets and chiral symmetry restoration in baryon spectrum," Phys. Lett. B 475, 329 (2000) [hep-ph/9908207].

[262] G. F. de Teramond, S. J. Brodsky and H. G. Dosch, Hadron spectroscopy and dynamics from light-front holography and conformal symmetry, arXiv:1401.5531 [hep-ph].

[263] D. D. Dietrich, P. Hoyer and M. Jarvinen, Boosting equal time bound states, Phys. Rev. D 85, 105016 (2012) [arXiv:1202.0826 [hep-ph]].

[264] P. Hoyer, Bound states - from QED to QCD, arXiv:1402.5005 [hep-ph].

[265] S. J. Brodsky and J. R. Primack, Electromagnetic interactions of loosely-bound composite systems, Phys. Rev. 174, 2071 (1968).

[266] S. J. Brodsky and J. R. Primack, The electromagnetic interactions of composite systems, Annals Phys. 52, 315 (1969).

[267] J. Segovia, L. Chang, I. C. Clot, C. D. Roberts, S. M. Schmidt and H. -s. Zong, Distribution amplitudes of light-quark mesons from lattice QCD, Phys. Lett. B 731, 13 (2014) [arXiv:1311.1390 [nucl-th]].

[268] F. Gao, L. Chang, Y. -X. Liu, C. D. Roberts and S. M. Schmidt, Parton distribution amplitudes of light vector mesons, arXiv:1405.0289 [nucl-th].

[269] M. Diehl, Generalized parton distributions from form factors, Nucl. Phys. Proc. Suppl. 161, 49 (2006) [arXiv:hep-ph/0510221].

[270] G. F. de Teramond, S. J. Brodsky, Light-front quantization and AdS/QCD: an overview, J. Phys. Conf. Ser. 287, 012007 (2011) [arXiv:1103.1100 [hep-ph]].

[271] R. Baldini et al., Nucleon time-like form factors below the $N \bar{N}$ threshold, Eur. Phys. J. C 11, 709 (1999).

[272] V. Tadevosyan et al., [Jefferson Lab $F_{\pi}$ Collaboration], Determination of the pion charge form factor for $Q^{2}=0.60-1.60 \mathrm{GeV}^{2}$, Phys. Rev. C 75, 055205 (2007) [arXiv:nucl-ex/0607007]; 
[273] T. Horn et al., [Jefferson Lab $F_{\pi}$ Collaboration], Determination of the charged pion form factor at $Q^{2}=1.60$ and $2.45(\mathrm{GeV} / \mathrm{c})^{2}$, Phys. Rev. Lett. 97, 192001 (2006) [arXiv: nucl-ex/0607005].

[274] S. Hong, S. Yoon and M. J. Strassler, On the couplings of vector mesons in AdS/QCD, JHEP 0604, 003 (2006) [arXiv:hep-th/0409118].

[275] H. R. Grigoryan and A. V. Radyushkin, Form factors and wave functions of vector mesons in holographic QCD, Phys. Lett. B 650, 421 (2007) [arXiv:hep-ph/0703069].

[276] H. R. Grigoryan and A. V. Radyushkin, Pion form factor in chiral limit of hard-wall AdS/QCD model, Phys. Rev. D 76, 115007 (2007) [arXiv:0709.0500 [hep-ph]].

[277] H. R. Grigoryan and A. V. Radyushkin, Pion in the holographic model with 5D Yang-Mills fields, Phys. Rev. D 78, 115008 (2008) [arXiv:0808.1243 [hep-ph]].

[278] H. J. Kwee and R. F. Lebed, Pion form factors in holographic QCD, JHEP 0801, 027 (2008) [arXiv:0708.4054 [hep-ph]].

[279] H. J. Kwee and R. F. Lebed, Pion form factor in improved holographic QCD backgrounds, Phys. Rev. D 77, 115007 (2008) [arXiv:0712.1811 [hep-ph]].

[280] H. R. Grigoryan and A. V. Radyushkin, Structure of vector mesons in a holographic model with linear confinement, Phys. Rev. D 76, 095007 (2007) [arXiv:0706.1543 [hep-ph]].

[281] J. J. Sakurai, Theory of strong interactions, Annals Phys. 11, 1 (1960).

[282] J. I. Friedman, Deep inelastic scattering: Comparisons with the quark model, Rev. Mod. Phys. 63, 615 (1991).

[283] P. Masjuan, E. Ruiz Arriola and W. Broniowski, Meson dominance of hadron form factors and large- $N_{C}$ phenomenology, Phys. Rev. D 87, 014005 (2013) [arXiv:1210.0760 [hep-ph]].

[284] S. J. Brodsky, F.-G. Cao and G. F. de Teramond, Meson transition form factors in light-front holographic QCD, Phys. Rev. D 84, 075012 (2011) [arXiv:1105.3999 [hep-ph]].

[285] J. H. Kuhn and A. Santamaria, Tau decays to pions, Z. Phys. C 48, 445 (1990). 
[286] C. Bruch, A. Khodjamirian and J. H. Kuhn, Modeling the pion and kaon form factors in the timelike region, Eur. Phys. J. C 39, 41 (2005) [arXiv: hep-ph/0409080].

[287] C. Hanhart, A new parameterization for the pion vector form factor, Phys. Lett. B 715, 170 (2012) [arXiv:1203.6839 [hep-ph]].

[288] D. Gómez Dumm and P. Roig, Dispersive representation of the pion vector form factor in $\tau \rightarrow \pi \pi \nu_{\tau}$ decays, Eur. Phys. J. C 73, 2528 (2013) [arXiv:1301.6973 [hep-ph]].

[289] N. N. Achasov and A. A. Kozhevnikov, Pion form factor and reactions $e^{+} e^{-} \rightarrow \omega \pi^{0}$ and $e^{+} e^{-} \rightarrow \pi^{+} \pi^{-} \pi^{+} \pi^{-}$at energies up to $23 \mathrm{GeV}$ in the many-channel approach, Phys. Rev. D 88, 093002 (2013) [arXiv:1305.6117 [hep-ph]].

[290] A. Ballon-Bayona, G. Krein and C. Miller, Decay constants of the pion and its excitations in holographic QCD, arXiv:1412.7505 [hep-ph].

[291] G. J. Gounaris and J. J. Sakurai, Finite width corrections to the vector meson dominance prediction for $\rho \rightarrow e^{+} e^{-}$, Phys. Rev. Lett. 21, 244 (1968).

[292] J. F. Gunion, S. J. Brodsky and R. Blankenbecler, Large-angle scattering and the interchange force, Phys. Rev. D 8, 287 (1973).

[293] B. R. Baller, G. C. Blazey, H. Courant, K. J. Heller, S. Heppelmann et al., Comparison of exclusive reactions at large $t$, Phys. Rev. Lett. 60, 1118 (1988).

[294] S. Brodsky, G. de Teramond and M. Karliner, Puzzles in hadronic physics and novel quantum chromodynamics phenomenology, Ann. Rev. Nucl. Part. Sci. 62, 1 (2012) [arXiv: 1302.5684 [hep-ph]].

[295] G. F. de Teramond and S. J. Brodsky, Gauge/gravity duality and strongly coupled light-front dynamics, PoS LC 2010, 029 (2010) [arXiv:1010.1204 [hep-ph]].

[296] F. Ambrosino et al. [KLOE Collaboration], Measurement of $\sigma\left(e^{+} e^{-} \rightarrow \pi^{+} \pi^{-} \gamma(\gamma)\right.$ and the dipion contribution to the muon anomaly with the KLOE detector, Phys. Lett. B 670, 285 (2009) [arXiv:0809.3950 [hep-ex]].

[297] F. Ambrosino et al. [KLOE Collaboration], Measurement of $\sigma\left(e^{+} e^{-} \rightarrow \pi^{+} \pi^{-}\right)$ from threshold to $0.85 \mathrm{GeV}^{2}$ using Initial State Radiation with the KLOE detector, Phys. Lett. B 700, 102 (2011) [arXiv:1006.5313 [hep-ex]]. 
[298] D. Babusci et al. [KLOE Collaboration], Precision measurement of $\sigma\left(e^{+} e^{-} \rightarrow\right.$ $\left.\pi^{+} \pi^{-} \gamma\right) / \sigma\left(e^{+} e^{-} \rightarrow \mu^{+} \mu^{-} \gamma\right)$ and determination of the $\pi^{+} \pi^{-}$contribution to the muon anomaly with the KLOE detector, Phys. Lett. B 720, 336 (2013) [arXiv:1212.4524 [hep-ex]].

[299] B. Aubert et al. [BABAR Collaboration], Precise measurement of the $e^{+} e^{-} \rightarrow$ $\pi^{+} \pi^{-}(\gamma)$ cross section with the initial-state radiation method at BABAR, Phys. Rev. Lett. 103, 231801 (2009) [arXiv:0908.3589 [hep-ex]].

[300] J. P. Lees et al. [BABAR Collaboration], Precise measurement of the $e^{+} e^{-} \rightarrow$ $\pi^{+} \pi^{-}(\gamma)$ cross section with the initial-state radiation method at BABAR, Phys. Rev. D 86, 032013 (2012) [arXiv:1205.2228 [hep-ex]].

[301] M. Fujikawa et al. [Belle Collaboration], High-statistics study of the $\tau^{-} \rightarrow \pi^{-} \pi^{0} \nu_{\tau}$ decay, Phys. Rev. D 78, 072006 (2008) [arXiv:0805.3773 [hep-ex]].

[302] K. K. Seth, S. Dobbs, et al., Electromagnetic structure of the proton, pion, and kaon by high-precision form factor measurements at large timelike momentum transfers, Phys. Rev. Lett. 110, 022002 (2013) [arXiv:1210.1596 [hep-ex]].

[303] S. Pacetti, R. Baldini Ferroli and E. Tomasi-Gustafsson, "Proton electromagnetic form factors: Basic notions, present achievements and future perspectives," Phys. Rept. 550-551, 1 (2014).

[304] H. Dahiya, A. Mukherjee and S. Ray, Parton distributions in impact parameter space, Phys. Rev. D 76, 034010 (2007) [arXiv:0705.3580 [hep-ph]].

[305] Z. Abidin and C. E. Carlson, Hadronic momentum densities in the transverse plane, Phys. Rev. D 78, 071502 (2008) [arXiv:0808.3097 [hep-ph]].

[306] A. Vega, I. Schmidt, T. Gutsche and V. E. Lyubovitskij, Generalized parton distributions in AdS/QCD, Phys. Rev. D 83, 036001 (2011) [arXiv:1010.2815 $[\mathrm{hep}-\mathrm{ph}]$.

[307] A. Vega, I. Schmidt, T. Gutsche and V. E. Lyubovitskij, Generalized parton distributions in an AdS/QCD hard-wall model, Phys. Rev. D 85, 096004 (2012) [arXiv:1202.4806 [hep-ph]].

[308] R. Nishio, T. Watari, Investigating generalized parton distribution in gravity dual, Phys. Lett. B 707, 362 (2012) [arXiv:1105.2907 [hep-ph]]. 
[309] A. Watanabe and K. Suzuki, Nucleon structure functions at small $x$ via the Pomeron exchange in AdS space with a soft infrared wall, Phys. Rev. D 89, 115015 (2014) [arXiv:1312.7114 [hep-ph]].

[310] M. Aghasyan, H. Avakian, E. De Sanctis et al., Studies of transverse momentum dependent parton distributions and Bessel weighting, arXiv:1409.0487 [hep-ph].

[311] G. F. de Teramond, in the 6th International Conference on Quarks and Nuclear Physics (QNP 2012), 16-20 Apr 2012, Palaiseau, France, http://qnp2012.sciencesconf.org/conference/qnp2012/Teramond.pdf.

[312] G. D. Cates, C. W. de Jager, S. Riordan and B. Wojtsekhowski, Flavor decomposition of the elastic nucleon electromagnetic form factors, Phys. Rev. Lett. 106, 252003 (2011) [arXiv:1103.1808 [nucl-ex]].

[313] T. Gutsche, V. E. Lyubovitskij, I. Schmidt and A. Vega, Light-front quark model consistent with Drell-Yan-West duality and quark counting rules, Phys. Rev. D 89, 054033 (2014) [arXiv:1306.0366 [hep-ph]].

[314] D. Chakrabarti and C. Mondal, Generalized parton distributions for the proton in AdS/QCD, Phys. Rev. D 88, 073006 (2013) [arXiv:1307.5128 [hep-ph]].

[315] D. Chakrabarti and C. Mondal, Nucleon and flavor form factors in a light front quark model in AdS/QCD, Eur. Phys. J. C 73, 2671 (2013) [arXiv:1307.7995 [hep-ph]].

[316] N. Kumar and H. Dahiya, Charge and magnetization densities in transverse coordinate and impact parameter space, Phys. Rev. D 90, 094030 (2014) [arXiv:1411.0817 [hep-ph]].

[317] N. Sharma, Generalized parton distributions in the soft-wall model of AdS/QCD, Phys. Rev. D 90, 095024 (2014) [arXiv:1411.7486 [hep-ph]].

[318] M. Lohmann, Calculation of neutral weak nucleon form factors with the AdS/QCD correspondence, M.S. Thesis, California State University, Long Beach (2014).

[319] G. F. de Teramond and S. J. Brodsky, Excited baryons in holographic QCD, AIP Conf. Proc. 1432, 168 (2012) [arXiv:1108.0965 [hep-ph]]. 
[320] H. R. Grigoryan, T.-S. H. Lee, H.-U. Yee, Electromagnetic nucleonto-delta transition in holographic QCD, Phys. Rev. D80, 055006 (2009) [arXiv: 0904.3710 [hep-ph]].

[321] A. Ballon-Bayona, H. Boschi-Filho, N. R. F. Braga, M. Ihl and M. A. C. Torres, Production of negative parity baryons in the holographic Sakai-Sugimoto model, Phys. Rev. D 86, 126002 (2012) [arXiv:1209.6020 [hep-ph]].

[322] K. -Y. Kim and I. Zahed, Electromagnetic baryon form factors from holographic QCD, JHEP 0809, 007 (2008) [arXiv:0807.0033 [hep-th]].

[323] C. A. Ballon-Bayona, H. Boschi-Filho, N. R. F. Braga, M. Ihl and M. A. C. Torres, Generalized baryon form factors and proton structure functions in the SakaiSugimoto model, Nucl. Phys. B 866, 124 (2013) [arXiv:1112.1439 [hep-ph]].

[324] M. A. B. Beg, B. W. Lee and A. Pais, SU(6) and electromagnetic interactions, Phys. Rev. Lett. 13, 514 (1964).

[325] R. Pohl, A. Antognini, F. Nez et al., The size of the proton, Nature 466, 213 (2010).

[326] M. A. B. Beg and A. Zepeda, Pion radius and isovector nucleon radii in the limit of small pion mass, Phys. Rev. D 6, 2912 (1972).

[327] S. Collins, M. Gockeler, P. Hagler et al., Dirac and Pauli form factors from lattice QCD, Phys. Rev. D 84, 074507 (2011) [arXiv:1106.3580 [hep-lat]].

[328] A. Karch and E. Katz, Adding flavor to AdS/CFT, JHEP 0206, 043 (2002) [arXiv:hep-th/0205236].

[329] C. Csaki, C. Grojean, H. Murayama, L. Pilo and J. Terning, Gauge theories on an interval: Unitarity without a Higgs, Phys. Rev. D 69, 055006 (2004) [arXiv:hep-ph/0305237].

[330] K. Agashe, R. Contino and A. Pomarol, The Minimal composite Higgs model, Nucl. Phys. B 719, 165 (2005) [arXiv:hep-ph/0412089].

[331] D. K. Hong and H. -U. Yee, Holographic estimate of oblique corrections for technicolor, Phys. Rev. D 74, 015011 (2006) [arXiv:hep-ph/0602177]. 
[332] J. Hirn and V. Sanz, A negative S parameter from holographic technicolor, Phys. Rev. Lett. 97, 121803 (2006) [arXiv:hep-ph/0606086].

[333] A. L. Fitzpatrick, J. Kaplan, E. Katz and L. Randall, Decoupling of high dimension operators from the low energy sector in holographic models, arXiv: 1304.3458 [hep-th].

[334] J. Hirn, N. Rius and V. Sanz, Geometric approach to condensates in holographic QCD, Phys. Rev. D 73, 085005 (2006) [arXiv:hep-ph/0512240].

[335] N. Arkani-Hamed, M. Porrati and L. Randall, Holography and phenomenology, JHEP 0108, 017 (2001) [arXiv:hep-th/0012148].

[336] M. Gell-Mann, R. J. Oakes and B. Renner, Behavior of current divergences under $S U(3) \times S U(3)$, Phys. Rev. 175, 2195 (1968).

[337] D. Albrecht, J. Erlich and R. J. Wilcox, Nonlinear boundary dynamics and chiral symmetry in holographic QCD, Phys. Rev. D 85, 114012 (2012) [arXiv:1112.5643 [hep-ph]].

[338] V. Balasubramanian, P. Kraus, A. E. Lawrence and S. P. Trivedi, Holographic probes of anti-de Sitter space-times, Phys. Rev. D 59, 104021 (1999) [arXiv:hep-th/9808017].

[339] I. R. Klebanov and E. Witten, AdS / CFT correspondence and symmetry breaking, Nucl. Phys. B 556, 89 (1999) [arXiv:hep-th/9905104].

[340] A. Cherman, T. D. Cohen and E. S. Werbos, The Chiral condensate in holographic models of QCD, Phys. Rev. C 79, 045203 (2009) [arXiv:0804.1096 [hep-ph]].

[341] Z. Abidin and C. E. Carlson, Strange hadrons and kaon-to-pion transition form factors from holography, Phys. Rev. D 80, 115010 (2009) [arXiv:0908.2452 [hep-ph]].

[342] M. Unsal, Quantum phase transitions and new scales in QCD-like theories, Phys. Rev. Lett. 102, 182002 (2009) [arXiv:0807.0466 [hep-th]].

[343] C. D. Carone, J. Erlich and M. Sher, Extra gauge invariance from an extra dimension, Phys. Rev. D 78, 015001 (2008) [arXiv:0802.3702 [hep-ph]]. 
[344] J. Schechter and H. Weigel, The Skyrme model for baryons, in Quantum Field Theory, edited by A. N. Mitra [arXiv:hep-ph/9907554].

[345] A. Pomarol and A. Wulzer, Baryon physics in holographic QCD, Nucl. Phys. B 809, 347 (2009) [arXiv:0807.0316 [hep-ph]].

[346] D. T. Son and M. A. Stephanov, QCD and dimensional deconstruction, Phys. Rev. D 69, 065020 (2004) [arXiv:hep-ph/0304182].

[347] J. Babington, J. Erdmenger, N. J. Evans, Z. Guralnik and I. Kirsch, Chiral symmetry breaking and pions in nonsupersymmetric gauge/gravity duals, Phys. Rev. D 69, 066007 (2004) [arXiv:hep-th/0306018].

[348] R. J. Duffin, On the characteristic matrices of covariant systems, Phys. Rev. 54, 1114 (1938).

[349] N. Kemmer, The particle aspect of meson theory, Proc. Roy. Soc. Lond. A 173, 91 (1939).

[350] G. Petiau, Thesis University of Paris (1936), published in Acad. Roy. de Belg., A. Sci. Mem. Collect. 16, N 2, 1 (1936).

[351] B. L. G. Bakker, A. Bassetto, S. J. Brodsky et al., Light-front quantum chromodynamics: A framework for the analysis of hadron physics, arXiv:1309.6333 [hep-ph].

[352] I. G. Aznauryan, A. Bashir, V. Braun et al., Studies of nucleon resonance structure in exclusive meson electroproduction, Int. J. Mod. Phys. E 22, 1330015 (2013) [arXiv:1212.4891 [nucl-th]].

[353] D. Ashery, Measurement of light-cone wave functions by diffractive dissociation, Nucl. Phys. Proc. Suppl. 161, 8 (2006) [arXiv:hep-ex/0511052].

[354] S. J. Brodsky, G. F. de Teramond and R. Shrock, Light-front holography and hadronization at the amplitude level, AIP Conf. Proc. 1056, 3 (2008) [arXiv:0807.2484 [hep-ph]].

[355] J. R. Hiller, Light-front holography and the light-front coupled-cluster method, arXiv:1402.0226 [hep-ph]. 
[356] J. P. Vary, H. Honkanen, J. Li et al., Hamiltonian light-front field theory in a basis function approach, Phys. Rev. C 81, 035205 (2010) [arXiv:0905.1411 [nucl-th]].

[357] T. Faulkner, H. Liu, J. McGreevy and D. Vegh, Emergent quantum criticality, Fermi surfaces, and $\mathrm{AdS}_{2}$, Phys. Rev. D 83, 125002 (2011) [arXiv:0907.2694 [hep-th]].

[358] A. W. Peet and J. Polchinski, UV-IR relations in AdS dynamics, Phys. Rev. D 59, 065011 (1999) [arXiv:hep-th/9809022].

[359] I. Heemskerk and J. Polchinski, Holographic and Wilsonian renormalization groups, JHEP 1106, 031 (2011) [arXiv:1010.1264 [hep-th]].

[360] T. Faulkner, H. Liu and M. Rangamani, Integrating out geometry: Holographic Wilsonian RG and the membrane paradigm, JHEP 1108, 051 (2011) [arXiv:1010.4036 [hep-th]].

[361] D. Elander, H. Isono and G. Mandal, Holographic Wilsonian flows and emergent fermions in extremal charged black holes, JHEP 1111, 155 (2011) [arXiv:1109.3366 [hep-th]].

[362] A. Almheiri and J. Polchinski, Models of $\mathrm{AdS}_{2}$ backreaction and holography, arXiv:1402.6334 [hep-th].

[363] G. F. de Teramond, H. G. Dosch and S. J. Brodsky, Baryon spectrum from superconformal quantum mechanics and its light-front holographic embedding, Phys. Rev. D 91, XXXXXX (2015) [arXiv:1411.5243 [hep-ph]].

[364] See: H. G. Dosch, G. F. de Teramond and S. J. Brodsky, Superconformal baryonmeson symmetry and light-front holographic QCD, arXiv:1501.00959 [hep-th].

[365] V. P. Akulov and A. I. Pashnev, Quantum superconformal model in (1,2) space, Theor. Math. Phys. 56, 862 (1983) [Teor. Mat. Fiz. 56, 344 (1983)].

[366] For a lucid introduction see: M. B. Green, J. H. Schwarz and E. Witten, Superstring Theory, Vol. 2 (Cambridge University Press, 1987), p. 271-277.

[367] C. A. Bayona and N. R. F. Braga, Anti-de Sitter boundary in Poincaré coordinates, Gen. Rel. Grav. 39, 1367 (2007) [arXiv:hep-th/0512182]. 
[368] T. Levine, Symmetry principles in physics: Applications to conformal symmetries and CT-AdS correspondence, Senior Thesis, Brown University (2002).

[369] Z. Abidin and C. E. Carlson, Gravitational form factors in the axial sector from an AdS/QCD model, Phys. Rev. D 77, 115021 (2008) [arXiv:0804.0214 [hep-ph]].

[370] I. Y. Kobzarev and L. B. Okun, Gravitational interaction of fermions, Zh. Eksp. Teor. Fiz. 43, 1904 (1962) [Sov. Phys. JETP 16, 1343 (1963)].

[371] O. V. Teryaev, Spin structure of nucleon and equivalence principle, arXiv:hep-ph/9904376.

[372] V. Balasubramanian, P. Kraus and A. E. Lawrence, Bulk versus boundary dynamics in anti-de Sitter spacetime, Phys. Rev. D 59, 046003 (1999) [arXiv:hep-th/9805171].

[373] Handbook of Mathematical Functions, edited by M. Abramowitz and I.A. Stegun (Dover Publications, 1964).

[374] H. G. Dosch, J. Kripfganz and M. G. Schmidt, Structure of hadron in Migdal's regularization scheme for QCD, Phys. Lett. B 70, 337 (1977).

[375] H. G. Dosch, J. Kripfganz and M. G. Schmidt, Hadronic structure functions and form factors in low-order QCD with Migdal regularization, Nuovo Cim. A 49, 151 (1979).

[376] S. Narison and V. I. Zakharov, Hints on the power corrections from current correlators in $x$-space, Phys. Lett. B 522, 266 (2001) [arXiv:hep-ph/0110141].

[377] A. L. Kataev, N. V. Krasnikov and A. A. Pivovarov, The use of the finite energetic sum rules for the calculation of the light quark masses, Phys. Lett. B 123, 93 (1983).

[378] S. G. Gorishnii, A. L. Kataev and S. A. Larin, Next-next-to-leading perturbative QCD corrections and light quark masses, Phys. Lett. B 135, 457 (1984).

[379] H. M. Ratsimbarison, Quark scalar current correlator in AdS/QCD approach, Nucl. Phys. Proc. Suppl. 207-208, 240 (2010) [arXiv:1009.4637 [hep-th]].

[380] G. Veneziano, Construction of a crossing-symmetric, Regge-behaved amplitude for linearly rising trajectories, Nuovo Cim. A 57, 190 (1968). 
[381] L. Infeld, On a new treatment of some eigenvalue problems, Phys. Rev. 59, 737 (1941).

[382] L. Infeld and T. E. Hull, The factorization method, Rev. Mod. Phys. 23, 21 (1951).

[383] M. Arik, M. Baykal and A. Baykal, Factorization method for $d$-dimensional isotropic harmonic oscillator and the generalized Laguerre polynomials, arXiv:0808.2289 [math-ph].

[384] W. S. l'Yi, Correlators of currents corresponding to the massive $p$-form fields in AdS/CFT correspondence, Phys. Lett. B 448, 218 (1999) [arXiv: hep-th/9811097].

[385] D. Z. Freedman, S. S. Gubser, K. Pilch and N. P. Warner, Renormalization group flows from holography-supersymmetry and a $c$-theorem, Adv. Theor. Math. Phys. 3, 363 (1999) [arXiv:hep-th/9904017].

[386] M. Henningson and K. Sfetsos, Spinors and the AdS/CFT correspondence, Phys. Lett. B 431, 63 (1998) [arXiv:hep-th/9803251].

[387] A. S. Koshelev and O. A. Rytchkov, Note on the massive Rarita-Schwinger field in the AdS/CFT correspondence, Phys. Lett. B 450, 368 (1999) [arXiv:hep-th/9812238]. 